\title{
LARGE-SCALE SEPARATION \\ OF AMINO ACIDS BY \\ CONTINUOUS DISPLACEMENT \\ CHROMATOGRAPHY
}

By

Joseph P. DeCarli II

Giorgio Carta

Charles H. Byers

October 1989

Work Performed Under Contract Number DE-AC05-84OR21400

Prepared For

Stanley F. Sobczynski

Program Manager, CE-14

Office of Industrial Programs

U.S. Department of Energy

Conservation and Renewable Energy

Office of Industrial Programs

U.S. Department of Energy

Conservation and Renewable Energy

Washington, D.C. 20585 


\title{
LARGE-SCALE SEPARATION OF AMINO ACIDS BY CONTINUOUS DISPLACEMENT CHROMATOGRAPHY
}

\author{
J. P. DeCarli II \\ G. Carta \\ C. H. Byers
}

\begin{abstract}
Continuous annular chromatography (CAC) is a developing technology that allows truly continuous chromatographic separations. Previous work has demonstrated the utility of this technology for the separation of various materials by isocratic elution on a bench scale. Novel applications and improved operation of the process were studied in this work, demonstrating that CAC is a versatile apparatus which is capable of separations at high throughput. Three specific separation systems were investigated. Pilot-scale separations at high loadings were performed using an industrial sugar mixture as an example of scale-up for isocratic separations. Bench-scale experiments of a low concentration metal ion mixture were performed to demonstrate stepwise elution, a chromatographic technique which decreases dilution and increases sorbent capacity. Finally, the separation of mixtures of amino acids by ion exchange was investigated to demonstrate the use of displacement development on the CAC. This technique, which perhaps has the most potential, when applied to the CAC allowed simultaneous separation and concentration of multicomponent mixtures on a continuous basis. Mathematical models were developed to describe the CAC performance and optimize the operating conditions. For all the systems investigated, the continuous separation performance of the CAC was found to be very nearly the same as the batchwise performance of conventional chromatography. The technology appears, thus, to be very promising for industrial applications.
\end{abstract}




\section{TABLE OF CONTENTS}

Page

ABSTRACT $\ldots \ldots \ldots \ldots \ldots \ldots \ldots \ldots \ldots \ldots \ldots \ldots \ldots \ldots \ldots \ldots \ldots \ldots \ldots \ldots$ iii

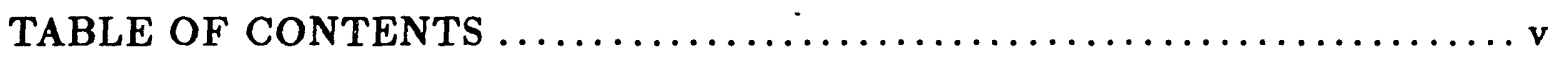

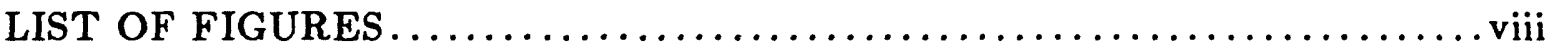

LIST OF TABLES $\ldots \ldots \ldots \ldots \ldots \ldots \ldots \ldots \ldots \ldots \ldots \ldots \ldots \ldots \ldots \ldots \ldots \ldots \ldots$

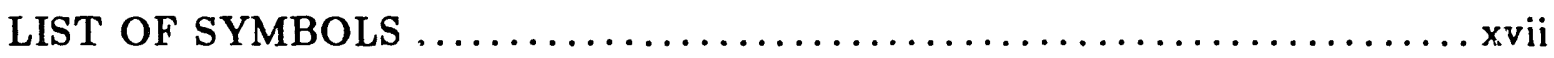

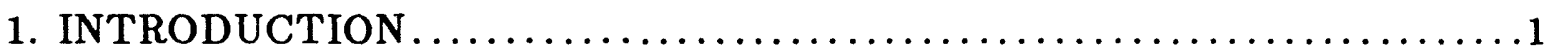

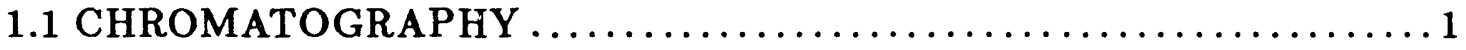

1.1.1 Isocratic Elution Chromatography $\ldots \ldots \ldots \ldots \ldots \ldots \ldots \ldots \ldots 2$

1.1.2 Stepwise and Gradient Elution $\ldots \ldots \ldots \ldots \ldots \ldots \ldots \ldots \ldots \ldots \ldots$

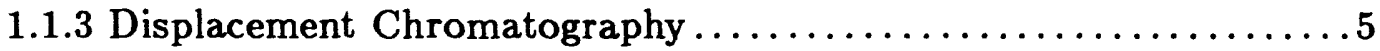

1.1.4 Frontal Analysis .................................. 6

1.2 CONTINUOUS CHROMATOGRAPHY $\ldots \ldots \ldots \ldots \ldots \ldots \ldots \ldots \ldots$

1.3 CONTINUOUS ANNULAR CHROMATOGRAPHY $\ldots \ldots \ldots \ldots \ldots 12$

1.4 ANALOGY BETWEEN FIXED BED AND CAC $\ldots \ldots \ldots \ldots \ldots \ldots 17$

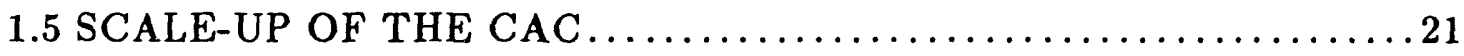

1.6 RESEARCH SCOPE AND APPROACH ...................24

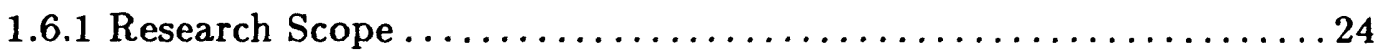

1.6.2 Research Approach ............................... 30

1.6.2.1 Isocratic Elution: Separation of Sugars ............... 30

1.6.2.2 Stepwise Elution: Separation of Metal Ions .............31

1.6.2.3 Displacement Development: Separation of Amino Acids .. 32

2. SEPARATION OF SUGARS BY ISOCRATIC ELUTION $\ldots \ldots \ldots \ldots \ldots . \ldots 34$

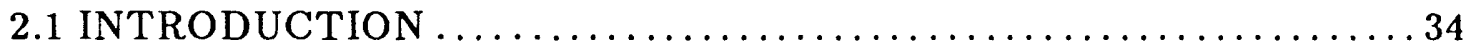


2.2 SUGAR SEPARATIONS BY ION EXCHANGE RESINS ........... 35

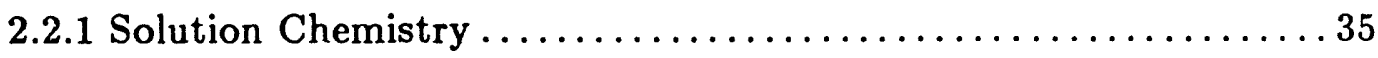

2.2.2 Chromatographic Separations .................... 37

2.3 INDUSTRIAL SUGARS: PRODUCTION AND SEPARATION ..... 41

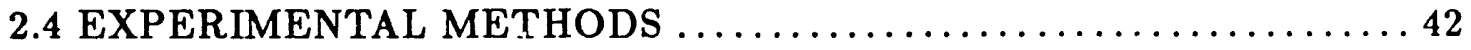

2.4.1 Equilibrium and Fixed-Bed Studies $\ldots \ldots \ldots \ldots \ldots \ldots \ldots \ldots, \ldots \ldots$

2.4.2 Continuous Annular Chromatographic Studies ........... 45

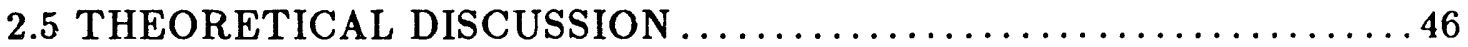

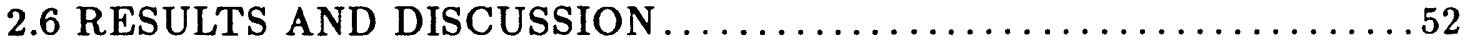

2.6.1 Equilibrium and Mass Transfer $\ldots \ldots \ldots \ldots \ldots \ldots \ldots \ldots \ldots$

2.6.2 Low Concentration - High Loading Experiments............ 57

2.6.3 High Concentration - Wide Feed Sector Experiments .........6 60

2.7 SUMMARY AND CONCLUSIONS $\ldots \ldots \ldots \ldots \ldots \ldots \ldots \ldots \ldots \ldots \ldots \ldots \ldots \ldots \ldots$

3. SEPARATION OF METALS BY STEP ELUTION . . . . . . . . . . . 69

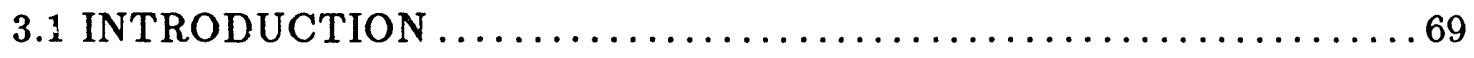

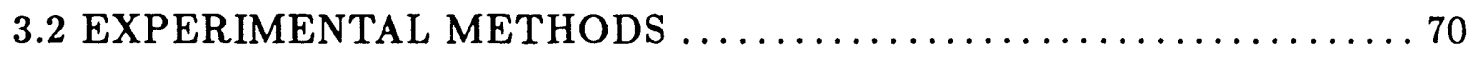

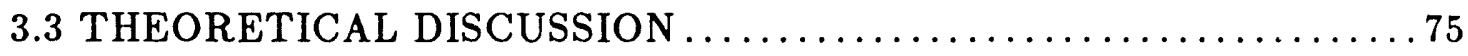

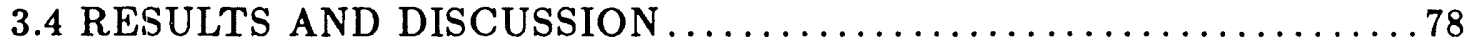

3.4.1 Fixed Bed Experiments $\ldots \ldots \ldots \ldots \ldots \ldots \ldots \ldots \ldots \ldots \ldots$

3.4.2 CAC Operation.......................... 80

3.5 SUMMARY AND CONCLUSIONS $\ldots \ldots \ldots \ldots \ldots \ldots \ldots \ldots \ldots \ldots \ldots$

4. SEPARATION OF AMINO ACIDS BY DISPLACEMENT

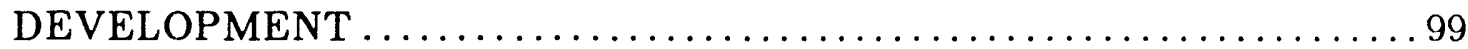

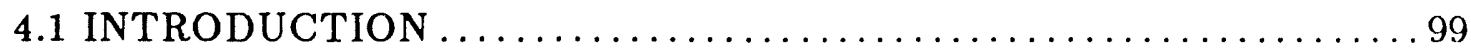

4.2 SEPARATION OF AMINO ACIDS BY ION EXCHANGE ........ 101 
4.3 EQUIPMENT AND EXPERIMENTAL METHODS.............. 103

4.3.1 Materials ...................................... 103

4.3.2 Equilibrium Uptake............................ 104

4.3.3 Fixed Bed and CAC Experiments .................. 106

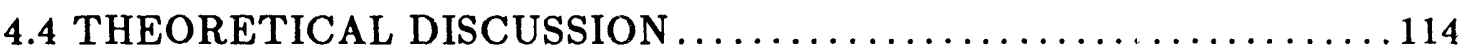

4.4.1 Solution Equilibrium $\ldots \ldots \ldots \ldots \ldots \ldots \ldots \ldots \ldots \ldots \ldots \ldots \ldots \ldots \ldots \ldots$

4.4.2 Uptake Equilibrium: Results and Modeling .............. 119

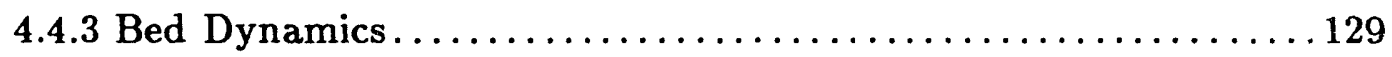

4.4.3.1 Glueckauf's Method .................... 136

4.4.3.2 Equilibrium Stage Model $\ldots \ldots \ldots \ldots \ldots \ldots \ldots \ldots \ldots \ldots 138$

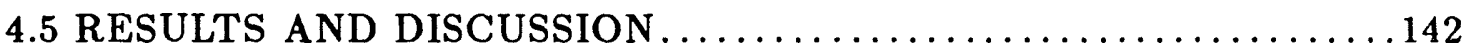

4.5.1 Fixed Bed Results .......................... 142

4.5.2 CAC Results ............................. 144

4.5.3 Displacer Concentration Effects ................... 149

4.5.4 Feed Concentration Effects ..................... 152

4.5.5 Rotation Rate Effects.............................. 161

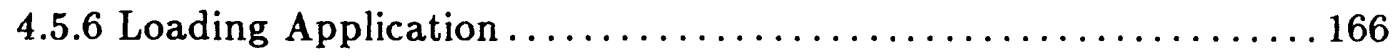

4.5.7 Nonbasic Displacer ................................... 169

4.6 SUMMARY AND CONCLUSIONS $\ldots \ldots \ldots \ldots \ldots \ldots \ldots \ldots \ldots \ldots \ldots \ldots$

5. CONCLUSIONS AND RECOMMENDATIONS $\ldots \ldots \ldots \ldots \ldots \ldots \ldots \ldots \ldots$

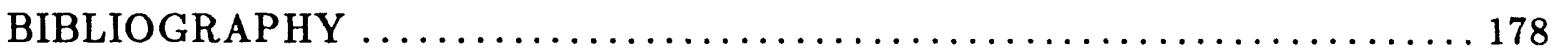




\section{LIST OF FIGURES}

Figure

Page

1.1 The principles of the three chromatographic methods:

(a) elution analysis, (b) displacement development, and

(c) frontal analysis. The left-hand side represents

a schematic of the column with sample passing

through it. The right-hand side shows the corresponding concentration history. A and B are

sample components while $\mathrm{C}$ is either the eluent or

the displacer (Ettre, 1980)

1.2.A Mechanically rotated radial flow separation

device (Siegell et al., 1986).

1.2.B Alternating crossflow separating device

(Siegell et al., 1986).

1.2.C Magnetically stabilized crossflow separating

device (Siegell et al., 1986).

1.3 Schematic illustrating moving-bed concept used

in the "Sorbex" Process. ........................... 12

1.4 Schematic illustrating principle of continuous annular chromatography (CAC)

1.5 Experimental and calculated dependence of Peclet number on Reynolds number from fixed-bed and CAC experiments (Howard et al., 1987).

1.6 Comparison of the analytical and orthogonal collocation solutions for the CAC (Howard et al., 1987).

1.7 Resolution between copper and nickel as a function of feed percentage in the CAC-II with the different annulus widths given in Table 1 (Begovich et al., 1983).... 25

1.8 Resolition between copper and nickel as a function of feed percentage in the CAC-ME with the different annulus widths given in Table 1 (Begovich et al., 1983).... 26 


\section{LIST OF FIGURES (continued)}

Figure

1.9 Resolution between copper and cobalt as a function of feed percentage in three different $\mathrm{CAC}$ units. The dimensions of the CAC-ME, the CAC-II, and the CAC-III are given in Table 1. (Begovich et al., 1982)......... 27

2.1 Mutarotation of $\alpha$ - and $\beta$-D-glucose.............. 36

2.2 Haworth projection formulas of $\alpha$ - and $\beta$-D-glucopyranose

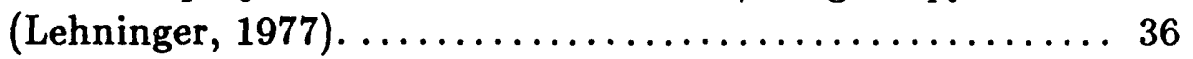

2.3 Haworth projection formulas of $\alpha$ - and $\beta$-D-fructopyranose

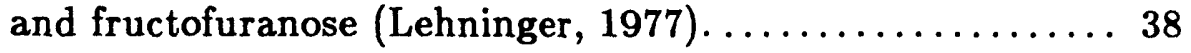

2.4 Conformational formulas of $\alpha$ - and $\beta$-D-glucopyranose(C1) and $\alpha$ - and $\beta$-D-fructopyranose (1C) (Lehninger, 1977;

Goulding, 1975).

Schematic of the fixed-bed apparatus

2.6 Construction detail of the CAC-ME. The unit has a 445-mm OD, an annulus width of $31.7 \mathrm{~mm}$, and a usable bed depth of $110 \mathrm{~cm} . \ldots \ldots \ldots \ldots \ldots \ldots \ldots \ldots \ldots \ldots \ldots$

2.8 Comparison of experimental fixed-bed results with the prediction of the simple mass transfer resistance theory (Eq. 2.4). Feed contains $25 \mathrm{~g} / \mathrm{L}$ glucose, 25 $\mathrm{g} / \mathrm{L}$ fructose, and $10 \mathrm{~g} / \mathrm{L}$ sucrose.

2.9 Comparison of experimental fixed-bed results with the equivalent run on the pilot-scale $\mathbb{C A C}$. A synthetic sugar mixture containing $25 \mathrm{~g} / \mathrm{L}$ glucose, $25 \mathrm{~g} / \mathrm{L}$ fructose, and $10 \mathrm{~g} / \mathrm{L}$ sucrose was used in both cases. The CAC feed also contained $1 \mathrm{~g} / \mathrm{L}$ of blue dextran and occupied a $3^{\circ}$ feed sector. The rotation rate was $360^{\circ} / \mathrm{h}$. 


\section{LIST OF FIGURES (continued)}

Figure

Page

2.10 Comparison of experimental $30^{\circ}$ feed-sector run with the theoretical predictions of Eq. 2.12. A diluted (10:1) industrial high-fructose corn syrup (HFCS) was used as the feed. The feed viscosity

was $1.12 \mathrm{cp}$, while that of the eluent was $0.97 \mathrm{cp}$, both measured at $25^{\circ} \mathrm{C}$. The rotation rate was $144^{\circ} / \mathrm{h} \ldots \ldots \ldots 60$

2.11 Comparison of experimental $55^{\circ}$ feed-sector run with the theoretical predictions of Eq. 2.12. A diluted (10:1) industrial high-fructose corn syrup was used as the feed. The rotation rate was $144^{\circ} / \mathrm{h} \ldots \ldots \ldots \ldots \ldots \ldots \ldots \ldots \ldots 6$

2.12 Comparison of experimental $30^{\circ}$ feed-sector run with the theoretical predictions of Eq. 2.12. A diluted (3:1) industrial HFCS was used as the feed. The feed viscosity was $2.2 \mathrm{cp}$, while that of the eluent was $0.97 \mathrm{cp}$, both measured at $25^{\circ} \mathrm{C}$. The rotation rate was $144^{\circ} / \mathrm{h} \ldots \ldots \ldots 63$

2.13 Comparison of experimental $30^{\circ}$ feed-sector run with the theoretical predictions of Eq. 2.12. A diluted (1:1) industrial HFCS was used as the feed. The feed viscosity was $2.76 \mathrm{cp}$, while that of the eluent was $0.97 \mathrm{cp}$, both measured at $25^{\circ} \mathrm{C}$. The rotation rate was $144^{\circ} / \mathrm{h} \ldots \ldots \ldots 64$

2.14 Comparison of experimental $30^{\circ}$ feed-sector rur with the theoretical predictions of Eq. 2.12. An undiluted industrial HFCS was used as the feed. The feed viscosity was $17.7 \mathrm{cp}$, while that of the eluent was $0.97 \mathrm{cp}$, both measured at $25^{\circ} \mathrm{C}$. The rotation rate was $144^{\circ} / \mathrm{h} \ldots \ldots \ldots 65$

2.15 Comparison of experimental CAC $30^{\circ}$ feed-sector run with an equivalent fixed-bed column run. An undiluted industrial HFCS was used as the feed. The feed viscosity was $17.7 \mathrm{cp}$, while that of the eluent was $0.97 \mathrm{cp}$, both measured at $25^{\circ} \mathrm{C}$ 


\section{LIST OF FIGURES (continued)}

Figure

2.16

3.1

3.2

3.3

3.4

3.8

3.10

Comparison of experimental $72^{\circ}$ feed-sector run with the theoretical predictions of Eq. 2.12. An undiluted industrial HFCS was used as the feed. The feed viscosity was $1.12 \mathrm{cp}$, while that of the eluent was $0.97 \mathrm{cp}$, both

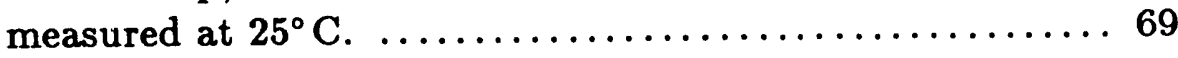

Laboratory-scale CAC unit.................. 74

Arrangement of feed and eluents in step-elution operation.. 77

Distribution coefficient as a function of the ammonium sulfate concentration. The eluent contains $0.025 \mathrm{M}$ $\mathrm{H}_{2} \mathrm{SO}_{4}$. Data at $\left[\left(\mathrm{NH}_{4}\right)_{2} \mathrm{SO}_{4}\right]=1.0 \underline{\mathrm{M}}$ include measurements at $0.5,1.0,5.0$, and $10.0 \mathrm{~g} / \mathrm{L} \mathrm{Fe}^{3+}$ and $\mathrm{Cr}^{3+} \ldots \ldots 82$

Comparison of experimental and calculated concentration profiles for a CAC isocratic run, using $0.4 \underline{\mathrm{M}}\left(\mathrm{NH}_{4}\right)_{2} \mathrm{SO}_{4}$ eluent.............................. 85

CAC step-elution separation of a mixture of ferric and chromic nitrates......................... 86

Comparison of CAC concentration profiles obtained for isocratic and step-elution operations............ 87

Effect of step-eluent concentration on CAC separation at base conditions (Table 3.2). Comparison of model and experimental results....................... 90

Peak concentrations and resolution obtained by varying the step eluent concentration at base conditions (Table 3.2)... 92

Effect of rotation rate on CAC separation at base conditions (Table 3.2). Comparison of model and experimental results........................ 93

Peak concentrations and resolution obtained by varying the rotation rate at base conditions (Table 3.2 )....... 94 


\section{LIST OF FIGURES (continued)}

Figure

3.11 Calculated effect of rotation rate on peak concentrations obtained at base conditions (Table 3.2) ........... 95

3.12 Peak concentrations and resolution obtained by varying the feed concentration at base conditions (Table 3.2).... 97

3.13 Peak concentrations and resolution obtained by varying the feed flow rate at base conditions (Table 3.2 )........ 98

3.14 Concentration profiles obtained in step-elution CAC separation of a dilute mixture of ferric and chromic nitrate. . . 100

4.1 General steps of an amino acid purification scheme (Reisman, 1977) 104

4.2 Simplified diagram of a CAC operating in the displacement mode.

4.3 Schematic of eluent distribution within the CAC operating in displacement mode.

4.4 Schematic of the equipment setup used in CAC displacement experiments......................

4.5 Photograph of CAC operating in the displacement mode.

(The band in the middle is amino acid.) ............. 119

4.6.A Glutamic acid ionic fraction as a function of $\mathrm{pH} . \ldots \ldots \ldots 123$

4.6.B Valine ionic fraction as a function of $\mathrm{pH} . \ldots \ldots \ldots \ldots \ldots 23$

4.6.C Leucine ionic fraction as a function of $\mathrm{pH} \ldots \ldots \ldots \ldots \ldots 124$

4.7.A Uptake of glutamic acid ions by cation-exchange resin Dowex 50 W-X8. Counter-ion: $\mathrm{H}^{+}$; Coion: $\mathrm{Cl}^{-} \ldots \ldots \ldots 126$

4.7.B Uptake of valine ions by cation-exchange resin Dowex 50W-X8. Counter-ion: $\mathrm{H}^{+}$; Coion: $\mathrm{Cl}^{-} \ldots \ldots \ldots \ldots \ldots \ldots \ldots$ 


\section{LIST OF FIGURES (continued)}

Figure

4.7.C Uptake of leucine ions by cation-exchange resin Dowex 50W-X8. Counter-ion: $\mathrm{H}^{+}$; Coion: $\mathrm{Cl}^{-} \ldots \ldots \ldots \ldots \ldots \ldots 127$

4.8.A Ion-exchange equilibrium for binary ion exchange of glutamic acid cations and hydrogen ions on Dowex 50W-X8. ..... 130

4.8.B Ion-exchange equilibrium for binary ion exchange of valine cations and hydrogen ions on Dowex 50W-X8.... 130

4.8.C Ion exchange equilibrium for binary ion exchange of leucine cations and hydrogen ions on Dowex 50W-X8. ... 131

4.9.A Comparison of constant and nonconstant separation factor isotherm for glutamic acid uptake equilibrium on Dowex 50W-X8.

4.9.B Comparison of constant and nonconstant separation factor isotherm for valine uptake equilibrium on Dowex 50W-X8.

4.9.C Comparison of constant and nonconstant separation factor isotherm for leucine uptake equilibrium on Dowex 50W-X8.

4.10.A Comparison of constant and nonconstant separation factors for binary ion-exchange equilibrium for glutamic acid cations and hydrogen ions on Dowex 50W-X8. ..... 140

4.10.B Comparison of constant and non-constant separation factors for binary ion exchange equilibrium for valine cations and hydrogen ions on Dowex 50W-X8. 140

4.i0.C Comparison of constant and non-constant separation factors for binary ion exchange equilibrium for leucine cations and hydrogen ions on Dowex 50W-X8. ... 141

4.11 Predicted outlet concentration by method of Glueckauf. . . 144

4.12 Comparison of experimental and calculated fixed-bed displacement chromatographic separation. 


\section{LIST OF FIGURES (continued)}

Figure

4.13 Comparison of experimental and calculated CAC displacement chromatographic separation at base conditions

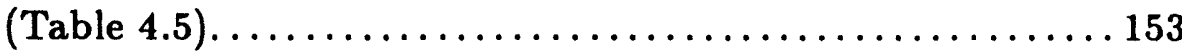

4.14 Comparison of experimental and calculated CAC displacement chromatographic separation at base conditions (Table 4.5) with an $80 \mathrm{mM} \mathrm{NaOH}$ displacer concentration.

Experimental and calculated values of the effect of $\mathrm{NaOH}$ displacer concentration on separation quality at base conditions (Table 4.5).

Experimental and calculated values of the effect of $\mathrm{NaOH}$ displacer concentration on average concentration at base conditions (Table 4.5) .................... 160

4.17 Experimental and calculated values of the effect of $\mathrm{NaOH}$ displacer concentration on peak concentration at base conditions (Table 4.5).

Experimental and calculated values of the effect of $\mathrm{NaOH}$ displacer concentration on peak concentration using Glueckauf's method.

Experimental and calculated values of the effect of feed conceritration on separation quality at base conditions (Table 4.5).

Comparison of experimental and calculated CAC displacement chromatographic separation at base conditions (Table 4.5) with a $10 \mathrm{~m} \underline{\mathrm{M}}$ feed concentration.

Experimental and calculated values of the effect of feed concentration on peak concentration at base conditions (Table 4.5). 


\section{LIST OF FIGURES (crntinued)}

Figure

Page

4.22 Experimental and calculated values of the effect of feed concentration on average concentration at base conditions (Table 4.5) . . . . . . . . . . . . . . . . 166

4.23 Experimental and calculated valuo of the effect of rotation rate on separation quality at base conditions (Table $\dot{x} .5$ ).

4.24 Experimental and calculated values of the effe $i$ of rotation rate on peak concentration at base conditions (Table 4.5).

4.25 Calculated values of glutamic acid ( $\square)$, valine $(+)$, and leucine $(-)$ at $a 10 \circ / \mathrm{h}$ rotation rate at base conditions (Table 4.5).

4.26 Experimental and calculated values of the effect of rotation rate on average concentration at base conditions (Table 4.5)

Calculated values of glutamic acid $(\square)$, valine $(+)$, and leucine (-) at the same loading with a concentratedfeed narrow feed sector (upper graph) and a dilute-feed wide feed sector (lower graph) at base conditions (Table 4.5).

Comparison of experime atal and calculated CAC displacement chromatographic separation at base conditions (Table 4.5) with a $75^{\circ}$ feed arc............ 176

Comparison of model predictions for fixed-bed displacement chromatographic separations at base conditions (Table 4.5) with a $100 \mathrm{mM} \mathrm{NaOH}$ and

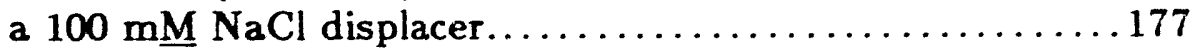




\section{LIST OF TABLES}

Tible

Page

1: Physical characteristics of the CAC units used at ORNL... 24

2: $\quad$ Equilibrium and mass transfer parameters for

Dowex $50 \mathrm{~W}-\mathrm{X8}(50 \mu \mathrm{m})$ in the calcium form..........56

3.1: $\quad$ Equilibrium and mass transfer parameters $\quad \ldots \ldots \ldots \ldots \ldots 4$

3.2: $\quad$ Base conditions for C.AC step-eluent experiments . $\quad \ldots \ldots 89$

4.1: $\quad$ Solution equilibrium parameters $\ldots \ldots \ldots \ldots \ldots \ldots \ldots \ldots$

4.2: Solubility of amino acids in water at their

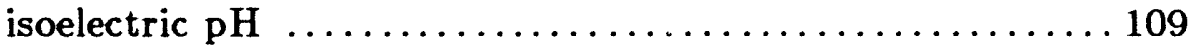

4.3: $\quad$ Ion exchange equilibrium parameters $\ldots \ldots \ldots \ldots \ldots \ldots$

4.4: Multicomponent ion exchange equilibrium on

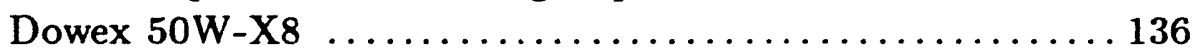

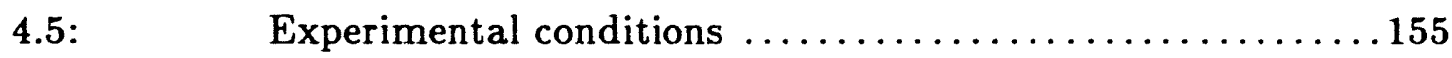




\section{LIST OF SYMBOLS}

a

$\frac{C}{C}$

$C^{*}$

$C_{F}$

$C_{s}^{0}$

$C^{1}$

$C_{\ell, j}$

$d_{p}$

$D_{z}$

$D_{\theta}$

$D_{e}$

$E_{\ell, j}$

$\bar{E}_{j}$

$\boldsymbol{k}^{\prime}$

$k$

$k_{o}$

$K$

$K_{1}$

$K_{2}$

$K_{3}$

$n$

$p$

$p_{\ell}$

$P e$

$q$

$q_{\max }$

$Q_{F}$

$Q_{T}$

$R$

$R_{0}$

$R e$

$S_{i, j}$

$S_{i, j}$

$t$

$\hat{t}$

$\hat{t}_{\max }$

$u$

$v$

$v_{c}$

interfacial area, $\mathrm{cm}^{-1}$

liquid-phase solute concentration, $\mathrm{g} / \mathrm{L}$

average liquid-phase solute concentration, $g / L$

equilibrium solute concentration, $\mathrm{g} / \mathrm{L}$

feed solute concentration, $\mathrm{g} / \mathrm{L}$

initial eluent concentration, $\mathrm{g} / \mathrm{L}$

step-eluent concentration, $\mathrm{g} / \mathrm{L}$

equilibrium constant

resin particle diameter, $\mathrm{cm}$

axial dispersion coefficient, $\mathrm{cm}^{2} / \mathrm{s}$

angular dispersion coefficient, $\mathrm{cm}^{2} / \mathrm{s}$

effective dispersion coefficient, $\mathrm{cm}^{2} / \mathrm{s}$

adsorption energy for ion $j$ on site $\ell, j / \mathrm{mol}$

average adsorption energy for ion $j, \mathrm{j} / \mathrm{mol}$

solid-phase mass transfer parameter, $\mathrm{s}^{-1}$

fluid-phase mass transfer coefficient, $\mathrm{cm} / \mathrm{s}$

global mass transfer coefficient, $\mathrm{cm} / \mathrm{s}$

equilibrium distribution coefficient

dissociation equilibrium constant, $\mathrm{mol} / \mathrm{L}$

dissociation equilibrium constant, $\mathrm{mol} / \mathrm{L}$

dissociation equilibrium constant, $\mathrm{mol} / \mathrm{L}$

number of transfer units $\left(k_{o} a z / u\right)$

skewness parameter in Eq. 4.30

probability distribution of site types $\ell$, Eq. 4.25

Peclet number $\left(d_{p} v / D_{e}\right)$

solid-phase average solute concentration, $\mathrm{mmol} / \mathrm{g}$ dry resin

solid-phase solute capacity, $\mathrm{mmol} / \mathrm{g}$ dry resin

feed flow rate, $\mathrm{cm}^{3} / \mathrm{min}$

total flow rate, $\mathrm{cm}^{3} / \mathrm{min}$

resolution, defined in Eq. 3.13

mean bed radius, $\mathrm{cm}$

Reynold number $\left(d_{p} u / \nu\right)$

separation factor for ion $i$ relative to ion $j, \mathrm{Eq} .4 .30$

average binary separation factor for ion $i$ relative to ion $j$, Eq. 4.31

time, $s$

chromatographic time, $\mathrm{s}$

peak chromatographic elution time, $\mathrm{s}$

superficial velocity, $\mathrm{cm} / \mathrm{s}$

interstial velocity, $\mathrm{cm} / \mathrm{s}$

component velocity, $\mathrm{cm} / \mathrm{s}$ 


$\begin{array}{ll}W_{i} & \text { exit bandwidth of component } i, \text { deg } \\ W_{i, j} & \text { heterogenity parameter, defined by Eq. } 4.32 \\ X & \text { liquid phase ionic fraction } \\ Y & \text { resin phase ionic fraction } \\ z & \text { bed axial position, } \mathrm{cm} \\ Z & \text { bed length, cm }\end{array}$

\section{Greek symbols}

$\alpha$

$\beta$

$\Delta$

$\epsilon$

$\frac{\theta}{\theta}$

$\bar{\theta}_{i}$

$\mu$

$\nu$

$\rho$

$\omega$

empirical constant in Eq. 3.12

empirical constant in Eq. 3.12

time interval at half of peak maximum concentration, $s$ bed voidage fraction

displacement from feed point, deg

peak elution angle of component $i, \mathrm{deg}$

fluid viscosity, $\mathrm{g}-\mathrm{cm} / \mathrm{s}$

fluid kinematic viscosity, $\mathrm{cm}^{2} / \mathrm{s}$

density, $\mathrm{g} / \mathrm{cm}^{3}$

rotation rate, $\mathrm{deg} / \mathrm{h}$ 


\section{LARGE-SCALE SEPARATION OF AMINO ACIDS BY CONTINUOUS DISPLACEMENT CHROMATOGRAPHY}

\section{J. P. DeCarli II}

G. Carta

C. H. Byers

\section{INTRODUCTION}

\subsection{CHROMATOGRAPHY}

Chromatography is a batch operation which allows the separation of multicomponent mixtures. In its simplest implementation, a small volume of the mixture to be separated is introduced to the top of a packed column. A carrier fluid is then continuously fed to the column causing the different solutes to travel down the column and eventually separate. The term chromatography is derived from the early use of this technique for the separation of plant pigments where the individual components could be identified by colorimetric tests (Ettre, 1980).

Different method of performing column chromatography were developed during World War II, mainly by Tiselius $(1940,1941,1943)$ and Claesson (1945, 1946, 1949). These techniques are classified as (1) elution chromatography (e.g., isocratic, stepwise, and gradient); (2) displacement chromatography; and (3) frontal analysis chromatography. Of these three approaches elution chromatography is widely used in the laboratory for analytical purposes; displacement development is used to separate large amounts of mixtures containing relatively few components; and frontal analysis, while of historical interest, has had little application in either analytical or process applications (Ettre, 1980). 


\subsubsection{Isocratic Elution Chromatography}

The conventional way of implementing elution chromatography is to inject a small sample of a mixture of components to be separated into the top of a chromatographic column and then elute it with a solvent. If the different components are retained by the sorbent to different extents, continuous feeding of the solvent (or eluent) will cause movement of the components at different velocities toward the column exit. The component velocity, $v_{c}$, is proportional to the eluent velocity and, for a dilute linear system, is related to the equilibrium distribution between mobile and stationary phases by the following equation:

$$
v_{c}=\frac{v}{1+\frac{1-\epsilon}{\epsilon} K},
$$

where $K$ is the equilibrium distribution coefficient, $\epsilon$ is the bed void fraction, and $v$ is the eluent interstitial velocity. If mass transfer resistances or other dispersive effects are present, $v_{c}$ merely represents the average component velocity. In this case, some molecules of the same species will be delayed while others will be hastened relative to the component velocity. The result is a spreading of the component band with concomitant dilution. Therefore, if mass transfer resistances are present, those components that have lower velocities stay in the column longer and become more diluted. When a single solvent is used to elute the feed mixture at a constant flow rate, without changing any other column conditions, the technique is called isocratic elution. The principle of elution development for the separation of components $\mathrm{A}$ and $\mathrm{B}$ with eluent $\mathrm{C}$ is illustrated in Fig. 1.1 (a) The major advantage of isocratic elution is that a multicomponent mixture may be completely separated into its pure components 
in a relatively easy fashion. Unfortunately, in some cases, the resulting dilution effect renders the technique unacceptable from the point of view of industrial processes. In some cases, however, separation of these same components can be carried out more efficiently using more complex techniques such as stepwise and gradient elution (sometimes referred to as solvent programming).

\subsubsection{Stepwise and Gradient Elution}

When two or more solvents are used to elute the components of a mixture the technique is called either stepwise or gradient elution. If the change between the solvents is abrupt, the method is called stepwise elution; if the change is gradual, the method is called gradient elution. In either case, the solvents are fed to the column in order of increasing eluting power. Changes in solution $\mathrm{pH}$, concentration, polarity, ionic strength, or any other thermodynamic variable that affects the distribution of solutes between stationary and mobile phases may be used.

Although simple elution can, in principle, completely separate mixtures into its pure components with little or no overlap, sometimes this technique is not the most efficient. For example, if the equilibrium distribution coefficients between the stationary and mobile phases for two components of a mixture are vastly different, the components will have vastly different retention times, resulting in a longer total run time. By increasing the solvent strength right after the first component has eluted from the column, the distribution coefficient of the second component can be decreased, and the species can be eluted from the column more quickly. The result is a reduction in the total run time. 
ORNL DWG 89A-562R
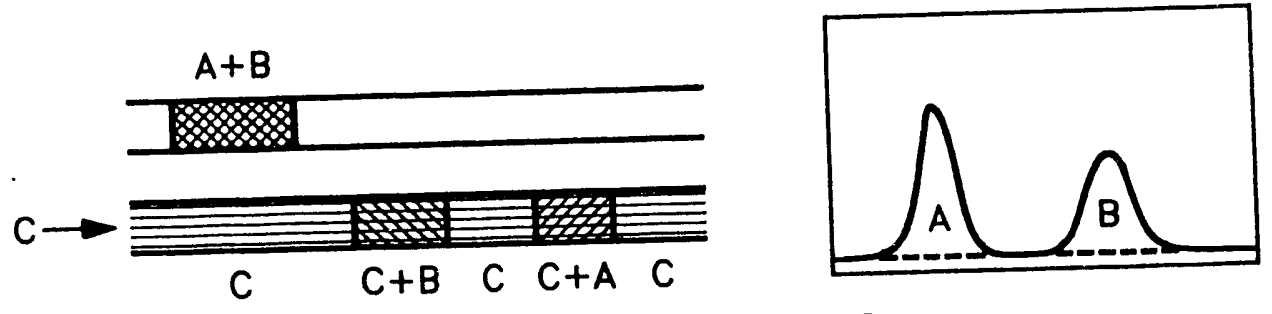

(a) ELUTION ANALYSIS
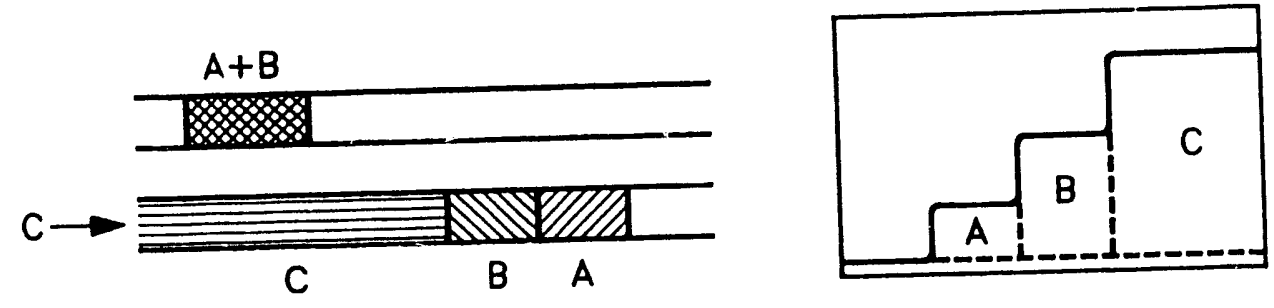

(b) DISPLACEMENT DEVELOPMENT

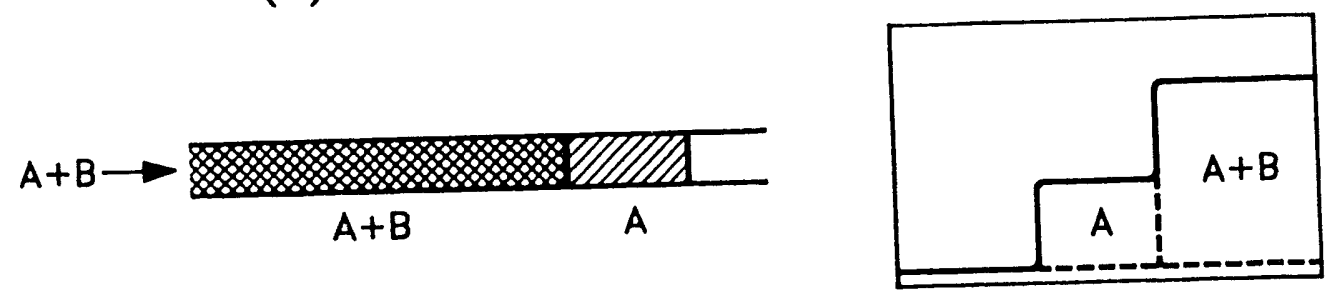

(c) FRONTAL ANALYSIS

Fig. 1.1 The principles of the three chromatographic methods: (a) elution analysis, (b) displacement development,and (c) frontal analysis. The left-hand side represents a schematic of the column with sample passing through it. The right-hand side shows the corresponding concentration history. $A$ and $B$ are sample components while C is either the eluent or the displacer (Ettre, 1980). 
On a large scale it is important because the latter case decreases the time between successive runs and increases productivity. Further benefits such as decreased eluent consumption and decreased product dilution might also be realized. For these reasons, stepwise or gradient elution techniques are often utilized when run time, eluent consumption, and product dilution are important considerations.

\subsubsection{Displacement Chromatography}

In displacement chromatography, a sample of a dilute mixture of components to be separated is introduced to the top of a chromatographic column. Introduction of the sample is followed by continual feeding of a solution containing a component (the displacer) that has an affinity for the stationary phase which is higher than the affinity of any component in the feed mixture. The displacer competes for adsorption sites with the feed components. If the displacer is present in a sufficiently high concentration, it will effectively desorb and concentrate the feed components. If mass transfer resistances are small, the various components will be distributed along the column length in order of decreasing affinity for the sorbent, with each upstream component acting as a "displacer" for each downstream species. The separated components finally exit the column in order of increasing affinity, with the displacer immediately following the feed components. The principle of displacement development for separating components $\mathrm{A}$ and $\mathrm{B}$ with displacer $\mathrm{C}$ is illustrated in Fig. 1.1 (b). This method allows better utilization of the sorbent capacity and accomplishes simultaneous separation and concentration of the feed mixture. In some instances, feed components 
may exceed their solubility in the eluting solution, causing crystallization in the packed bed. This is an important industrial problem, and its occurrence will depend on the concentration of the displacer. On the other hand, as shown in Fig. 1.1 (b), there is no separation between the product bands, so some mixing will inevitably occur. If high-purity fractions are required, the product yield is reduced. Another important consideration in displacement chromatography is the bed length. As long as the chromatographic bed is long enough for "isotachic" (i.e., constant velocity) concentrations to be reached, further increases in bed length can actually be detrimental to the separation performance. This is an important difference between the elution techniques described earlier and the displacement technique described here. For example, if the separation between a two-component mixture by isocratic elution is incomplete, $\mathrm{i} !$ can be improved by increasing the bed length. This will, of course, result in more product dilution. In displacement development, once isotachic concentrations have been reached, the separation between any two components cannot be improved by increasing column length.

\subsubsection{Frontal Analysis}

In frontal analysis, illustrated in Fig. 1.1 (c), a mixture of components to be separated, $A$ and $B$, is continuously fed into the column during the entire process. Pure component $A$, the least retained species, exits the column first with a sharp front after saturating the adsorbent. When the region of pure component A has eluted completely, it is followed by a sharp front containing a region with components $\mathbf{A}$ and $\mathbf{B}$. (For a three-component mixture, a region 
with all three components would follow). A major drawback of this technique is that it does not allow the isolation of different species, the major purpose of chromatography. For this reason it is infrequently used.

To summarize elution chromatography is used when complete separation of a small amount of mixture is desired; displacement development is used for the simultaneous separation and concentration of large amounts of mixtures, and frontal analysis may be used when only the least sorbed species is desired in pure form. For this reason, elution chromatography is custumarily used for analytical HPLC separations, and displacement development, which more efficiently utilizes the stationary phase, is the preferred method for preparative and industrial chromatography (Snyder and Kirkland, 1974; Mikes, 1979).

\subsection{CONTINUOUS CHROMATOGRAPHY}

The techniques of chromatography described above are of universal applicability provided that suitable chromatographic media and eluents can be found. While the efficiency of various sorbents varies, the process implementation of chromatography is generally based on the batch operations described in Sect.

\section{1 .}

Continuous operation, on the other hand, may be desirable for certain industrial applications. A few attempts have been made in the past to develop continuous chromatographic systems which do not suffer from the problems associated with batch processing (Tuthill, 1970). A few ways of implementing continuous or semicontinuous processing are illustrated in Figs. 1.2 (a), 1.2 (b), and $1.2(\mathrm{c})$. 
In Fig. 1.2 (a) continuous separation is achieved in the radial direction in a radial disc bed. The mixture of components to be separated is continuously fed to the center of the disc. As the disc rotates, the different components exit at different angular positions because of their different equilibrium distribution coefficients in the solvent. Here, the least retained component, A, exits first, followed by component $\mathrm{B}$ and then $\mathrm{C}$, the most retained component.

In Fig. 1.2 (b), a sorbent is packed between two solid, flat plates with porous edges that retain the sorbent. The feed mixture is continuously fed at the lower left-hand corner. The eluent initially flows in the same direction as the feed. Then, after a short period of time, the eluent flow in the feed direction is stopped and restarted in the direction transverse to the feed. In this way, a continuous crossflow contacting system is simulated.

Fig. 1.2 (c) describes a true moving bed. In this case, magnetic particles used as the sorbent are fed into one end of a rectangular bed. Air is used to fluidize the bed, and a magnet is used to keep the particles stabilized as they continuously move past the feed point and are recycled. A stationary feed point is used to continuously feed the gas stream to be separated. Continuous separation of this gas stream is accomplished by the air flow, the bed movement, and differences in the affinities of the components for the sorbent.

In these types of contacting systems there is constant motion of the sorbent relative to the feed point. This can be accomplished by either moving the bed and keeping the feed points stationary, or moving the fesd points and keeping the bed stationary. In addition, this motion can be designed such that the feed flow and the bed movement may be in a countercurrent or crosscurrent direction. Those systems with truly constant motion between the inlet points 

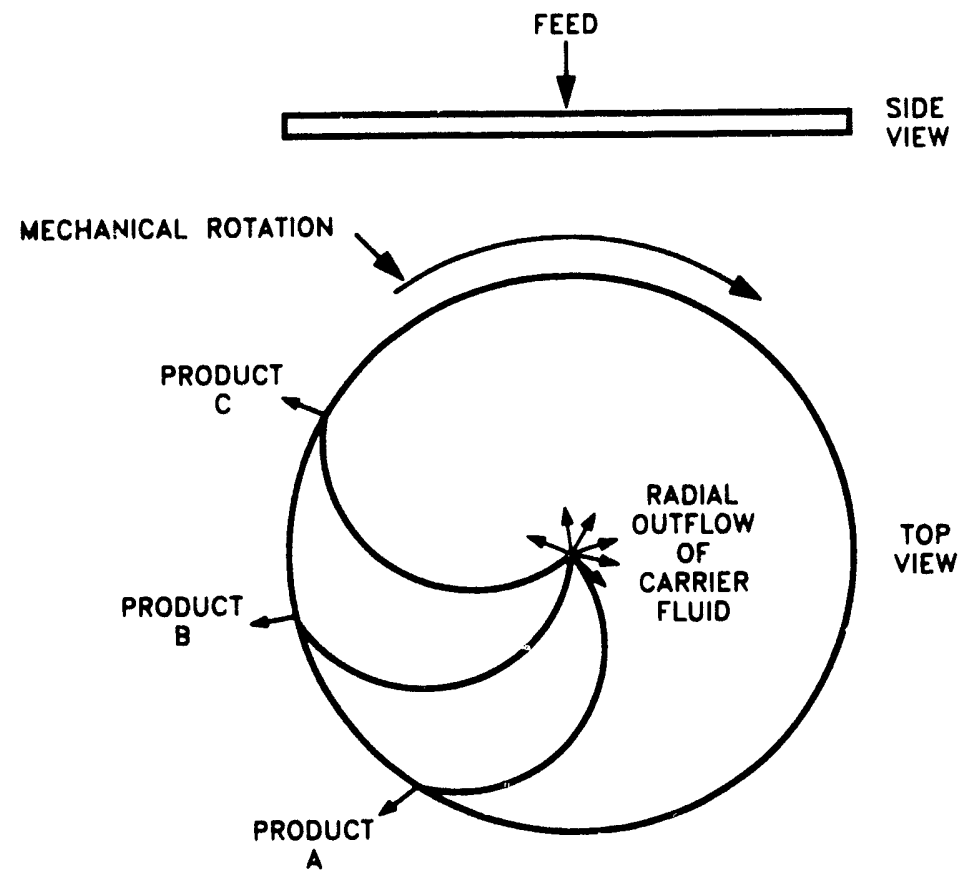

Fig. 1.2.a Mechanically rotated radial flow separation device (Siegell et al., 1986).

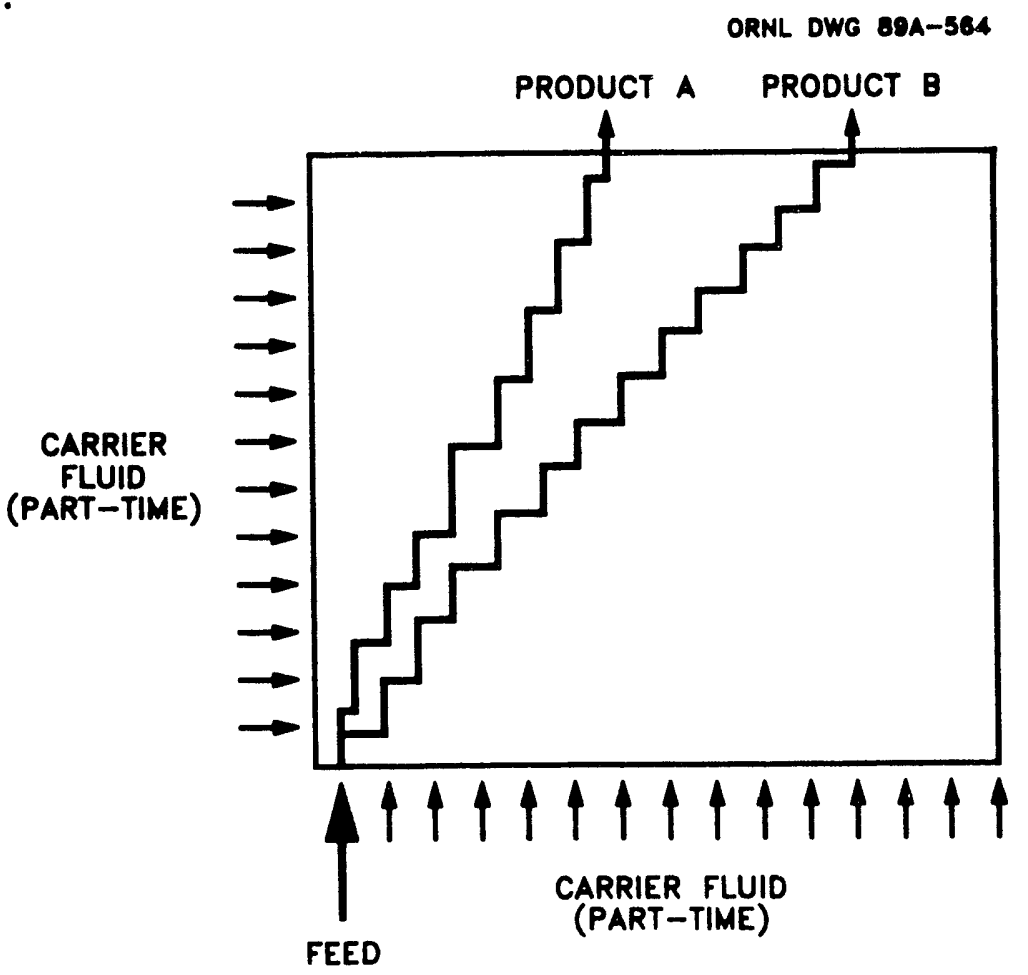

Fig. 1.2.b Alternating crossflow separating device (Siegell et al., 1986). 


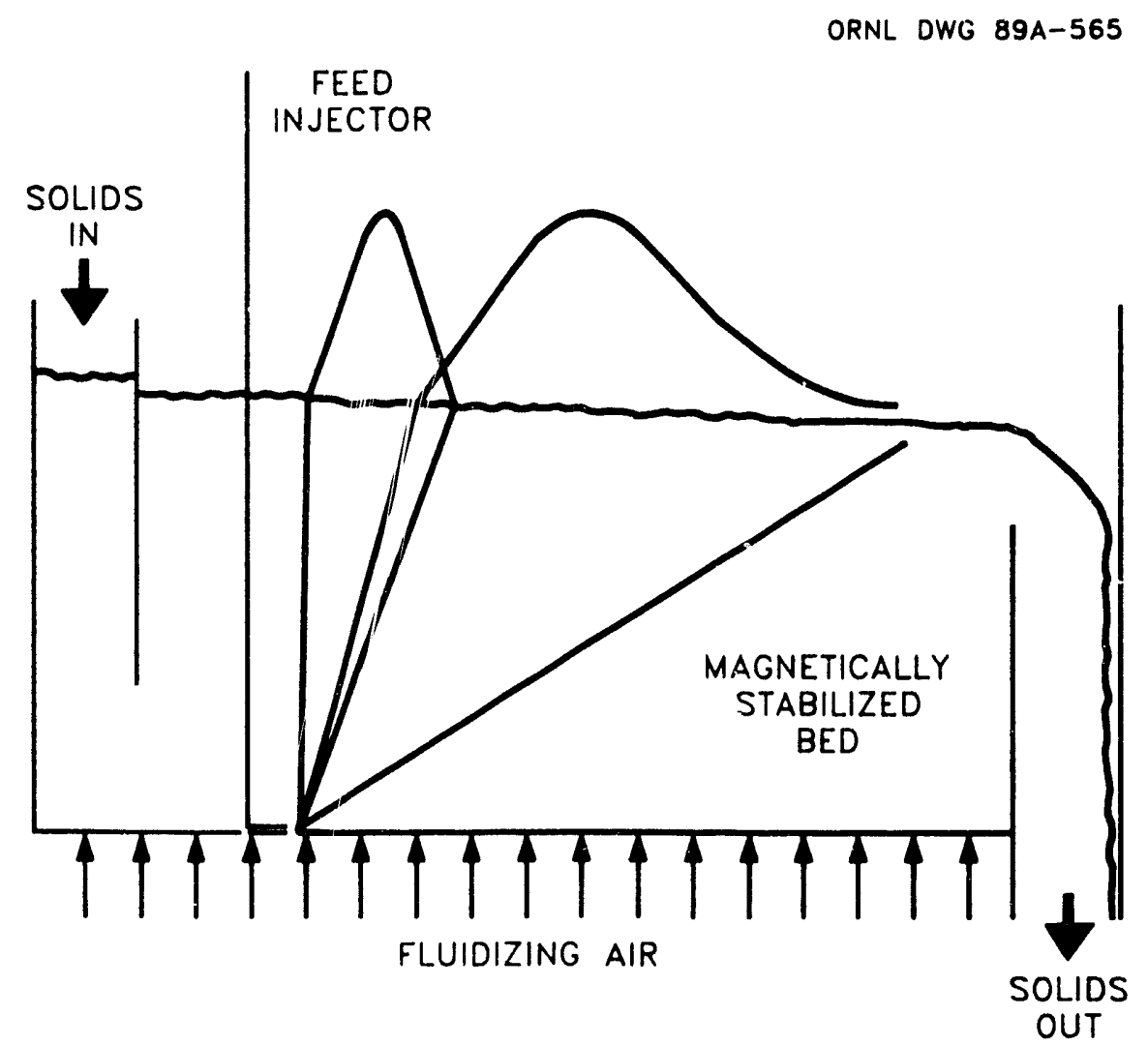

Fig. 1.2.c Magrietically stabilized crossflow separating device (Siegell et al., 1986). 
and the bed will be continuous, while those that simulate this motion will be semicontinuous. A semicontinuous process can approach the continuous process by making smaller and smaller discrete movements. For instance, in a cascade of fixed beds operated in a merry-go-round sequence, product can be collected from one bed and feed fed to another while the remaining columns are at an intermediate stage of the process. If timed in such a way that product is always being collected at one of the different column outlets, an apparently continuous process exists. When operated in a countercurrent manner in an arrangement resembling a distillation tower, binary separations can be obtained in a single unit. Thus $N-1$ columns are required to completely separate a mixture of $N$ components.

One such process, developed by UOP, Inc., is called "Sorbex" and is used for a variety of industrial applirations. The name of the particular proc ess depends on the material to be separated. For example, the separation of xylenes is called Parex, the separation of olefins is called Olex, the separation of n-paraffins is called Molex, and that of sugars is called Sarex (Sussman and Rathore, 1975). The Sorbex process is a simulated countercurrent system where the solid adsorbent is moved semicontinuously countercurrent to the liquid process stream. A conceptual view of the corresponciing idealized moving-bed process is illustrated in Fig. 1.3. For the separation of a two-component ( $A$ and $B$ ) system the system contains four zones defined by the introduction and withdrawal of process liquid. In zone $\mathrm{I}$, the sorbent adsorbs component $\mathrm{A}$; in zone II, component $B$ is desorbed; in zone III, the desorbent D strips component A from the sorbent; and finally, in zone IV, B replaces the desorbent D on the sorbent. The feed, $A$ and $B$, enters between zones $I$ and II where the more strongly retained species, 
$A$, is carried upward by the sorbent. In the upper portion of the column, $\mathbf{A}$ is stripped oír and exits at the top of the column. The more weakly retained component, B, moves downward where it is stripped and finally exits at the bottom of the column. In the actual Sorbex process, the sorbent remains stationary and the liquid feed and withdrawal points are moved through a cascade of fixed beds using a rotary valve system to simulate the sorbent movement. In this way, the difficulties of solids handling are avoided (Byers and Holmes, 1988; Barker and Ganetsos, 1987; Ching and Ruthven, 1984). The countercurrent action, whether real or simulated, provides a very efficient utilization of the sorbent and minimizes product dilution. Only binary separations are, however, possible with the Sorbex arrangement.

Crossflow systems, on the other hand, permit the separation of multicomponent mixtures in a single column. In this case the sorbent "moves" in a direction perpendicular to that of the fluid relative to the feed point. One such system, known as the continuous annular chromatograph, is the subject of the present study.

\subsection{CONTINUOUS ANNULAR CHROMATOGRAPHY}

The continuous annular chromatographic apparatus is illustrated in Fig. 1.4. In this apparatus, the sorbent is packed in the space between two concentric cylinders. For isocratic operation, the eluent is uniformly distributed thrrughout the annulus and flows downward toward the bottom of the bed. The feed mixture to be separated is fed at the top of the annular bed at a point that remains fixed in space while the entire bed assembly is slowly rotated on its 
ORHL OWC 88-1363

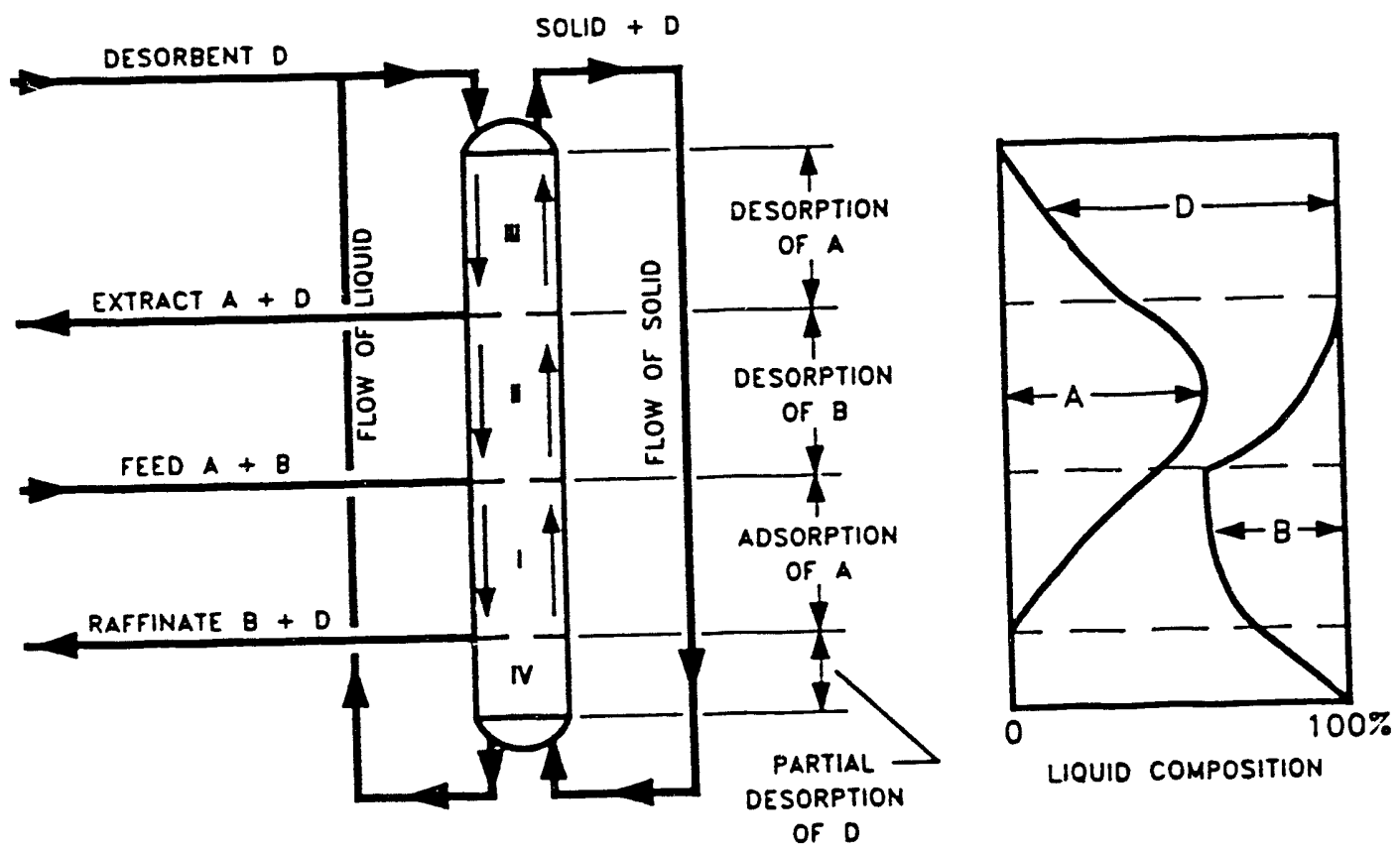

Fig. 1.3 Schematic illustrating moving-bed concept used in the "Sorbex" process. 
vertical axis. If the feed components have different affinities for the sorbent, they will travel along paths with different slopes, exiting at different angular locations relative to the feed point. The least-retained components will emerge closest to the feed port, while the more- retained components will exit at increasing angles from the feed port in the direction of rotation. In a few words, one can say that continuous annular chromatography, or $\mathrm{CAC}$, replaces the one-dimensional, unsteady-state process of conventional chromatography with a two-dimensional, steady-state process. In conventional chromatography the separated components exit at different times; here they exit at different angles.

The idea of CAC was first proposed by Martin (1949) and various conceptual. designs have been proposed since then. Dinelli et al. built a rotating unit for preparative-scale gas chromatography, which uses 100 fixed columns on a rotating carousel. Giddings (1961) demonstrated theoretically how the rotating annular column can be superior to a fixed column of the same volume for process scale applications. He recognized that many industrial packed tower operations exhibit nonuniformities in flow at large diameters, resulting in an increased plate height and loss of resolution. By using a rotating column with the same total cross-sectional area and bed height as a fixed column, but with an annulus size small enough so that flow nonuniformities do not occur, a process can be scaled up without loss of resolution. In other words, all things being equal, the geometry of a rotating bed with a small annulus will have a larger number of stages, resulting in better resolution, than the equivalent fixed bed. He also pointed out that process control might be easier in continu us operation because it results from the column geometry, and not from careful timing of feed injection and product withdrawal as in a simulated continuous operation. 
ORNL-DWG 81-15648R

FIXED

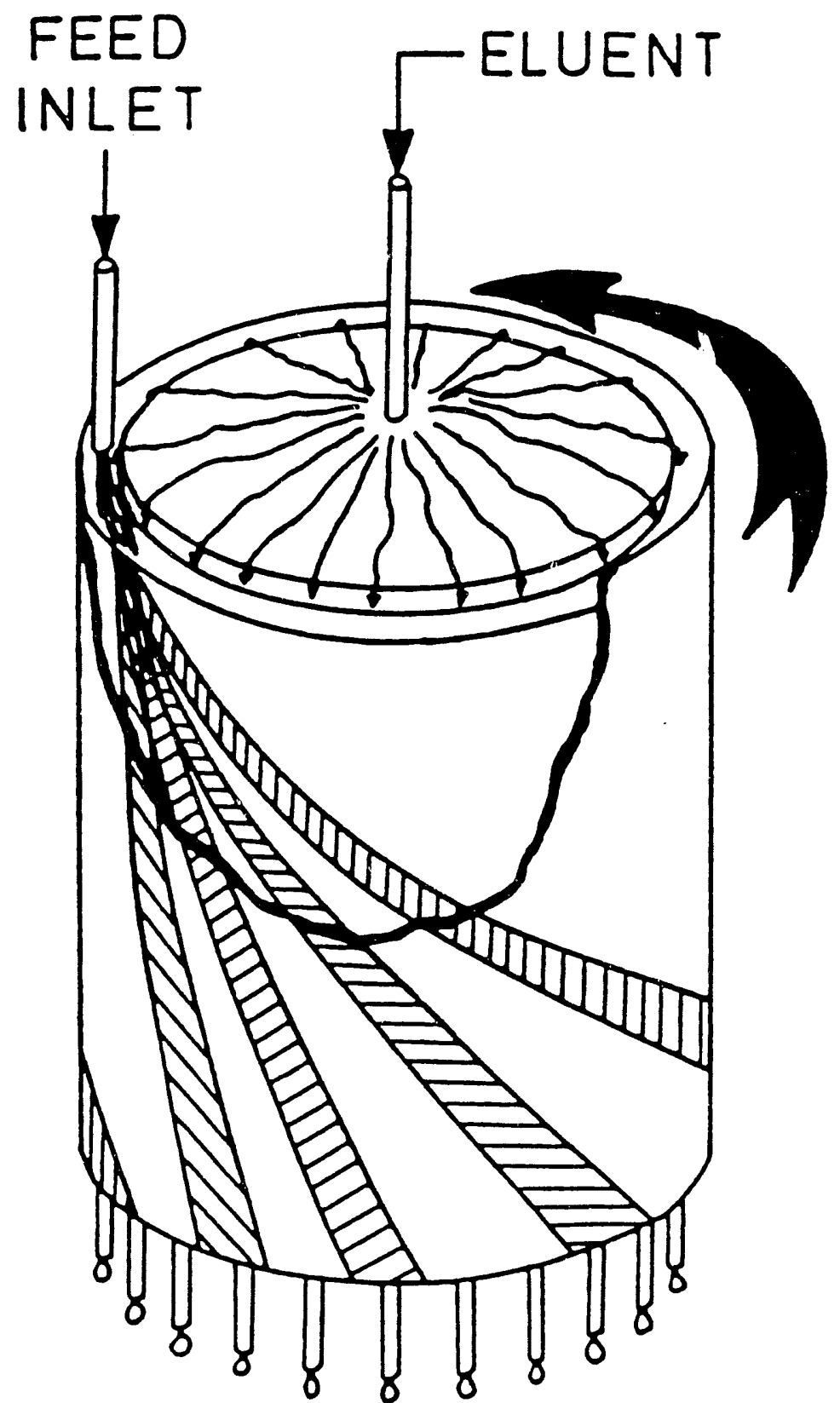

Fig. 1.4 Schematic illustrating principle of continuous annular chromatography ( $\mathrm{CAC})$. 
Carousels of columns similar to those of Dinelli and his coworkers (1962) have been built for liquid chromatographic operations by Byalyi and Ganitskii (1969), Witcherle and Coupek (1974), and Dunnill and Lilly (1972). Fox et al. (1969) and Moskvin and coworkers (1974A, 1974B, 1975) even constructed chromatographs similar to the one shown in Fig. 1.4.

However, the first major development that permitted large throughputs was the implementation of pressurization to the previous gravity-flow devices. The first successful prototype was developed in 1974 by Scott and coworkers (Scott et al., 1976) at the Oak Ridge National Laboratory (ORNL). The first experiments demonstrated the separation of blue dextran 2000 (a soluble dextran with a molecular weight of $2,000,000$ daltons) and cobalt chloride on a size-exclusion resin, and the separation of nickel-cobalt ions on a cation-exchange resin. Since then, the majority of the systems studied by ion exchange at ORNL have been mixtures of low-concentration metal ions. In addition, various process improvements, on the CAC.

Canon and Sisson (1978) developed an improved inlet distribution system which used a top layer of glass beads to prevent mixing of inlet fluids. They also successfully executed a preliminary experiment demonstrating the use of stepwise elution with the nickel-cobalt system. Canon et al. (1980) demonstrated the use of stepwise elution for the separation of iron-aluminum and zirconiumhafnium mixtures. The next major contribution, by Begovich et al. (1983), studied the effects of increasing the annulus width and bed diameter. The resolution of copper-nickel and nickel-cobalt mixtures was studied in different CAC units. The most recent work, by Howard et al. (1988), demonstrated the separation of dilute glucose-fructose-sucrose mixtures. In addition, this work 
also provided a more fundamental approach to modeling that is consistent with current chromatographic theories.

\subsection{ANJALOGY BETWEEN FIXED BED AND CAC}

In fixed-bed columns, the fluid and solid phase concentrations are functions of buth positicn and time. Considering a conventional, idealized, stationary bed with void fraction $\epsilon$, a one-dimensional, steady-state, material balance for a solute with concentration $C$ may be written as

$$
\epsilon D_{z} \frac{\partial^{2} C}{\partial z^{2}}=\epsilon \frac{\partial C}{\partial t}+(1-\epsilon) \frac{\partial q}{\partial t}+u \frac{\partial C}{\partial z}
$$

where $D_{z}$ is the axial dispersion coefficient; $u$ is the superficial velocity; and $C$ and $q$ are the liquid and solid phase concentrations, respectively. Using a simple fluid film model to describe fluid-particle mass transfer, the following rate equation may be written to relate the fluid and solid phase concentrations (Ruthven, 1984):

$$
(1-\epsilon) \frac{\partial q}{\partial t}=k_{o} a\left(C-C^{*}\right)
$$

where $k_{o} a$ is an overall mass transfer coefficient, and $C^{*}$ is the liquid phase concentration in equilibrium with the solid phase.

Continuity and rate equations can also be written in a cylindrical coordinate system for the two-dimensional CAC. Assuming steady state and neglecting velocity and concentration variations in the radial direction, these equations may be written as 


$$
\epsilon D_{z} \frac{\partial^{2} C}{\partial z^{2}}+\frac{\epsilon D_{\theta}}{R_{0}^{2}} \frac{\partial^{2} C}{\partial \theta^{2}}=\omega \epsilon \frac{\partial C}{\partial \theta}+\omega(1-\epsilon) \frac{\partial q}{\partial \theta}+u \frac{\partial C}{\partial z}
$$

and

$$
\omega(1-\epsilon) \frac{\partial q}{\partial \theta}=k_{o} a\left(C-C^{*}\right)
$$

where $D_{z}$ and $D_{\theta}$ are the axial and angular dispersion coefficients; $R_{0}$ is the mean radius of the annular bed; $\omega$ is the rate of rotation; and $z$ and $\theta$ are the axial and angular coordinates, respectively. (An additional assumption is implicitly made here that the annulus is "thin" and that axial and angular dispersion may be represented by Fickian diffusion terms.) If angular dispersion is negligible, then the one-dimensional, unsteady-state, fixed-bed equations (Eqs. 1.2 and 1.3) can be transformed into the corresponding steady-state, two-dimensional, continuous equations (1.4 and 1.5) with the change of variable:

$$
\theta=\omega t^{\prime}
$$

where $\omega$ is the rotation rate, $t^{\prime}$ is a transformed time, and $\theta$ is the angle (Wankat, 1977; Rhee et al., 1970; Begovich, 1982). Equations 1.4 and 1.5 become

$$
\epsilon D_{z} \frac{\partial^{2} C}{\partial z^{2}}=\epsilon \frac{\partial C}{\partial t^{\prime}}+(1-\epsilon) \frac{\partial q}{\partial t^{\prime}}+u \frac{\partial C}{\partial z}
$$

and

$$
(1-\epsilon) \frac{\partial q}{\partial t^{\prime}}=k_{o} a\left(C-C^{*}\right)
$$

These equations can be solved with the appropriate boundary conditions. For isocratic operation, for example, the boundary conditions may be written as 


$$
\begin{array}{lll}
t^{\prime}=0, & \text { all } z: & q=c=0 \\
z=0, & 0<t^{\prime} \leq t_{F}^{\prime}, C-\frac{\epsilon D_{F}}{u} \frac{\partial C}{\partial z}=C_{F} \\
& t^{\prime}>t_{F}^{\prime}, \quad & C-\frac{\epsilon D_{F}}{u} \frac{\partial C}{\partial z}=0 \\
z=Z, & \text { all } t^{\prime}: & \frac{\partial C}{\partial z}=0
\end{array}
$$

where $C_{F}$ is the feed concentration and $t_{F}^{\prime}$ is the length of time corresponding to the feed $\operatorname{arc} \theta_{F}=\omega t_{F}^{\prime}$.

Because of this analogy, the mathematical treatment of the steady-state performance of the $\mathrm{CAC}$ is not more complicated than the corresponding mathematical treatment of the analogous transient conventional chromatographic operation. Thus, solutions that are available to describe the latter can be used very simply to describe the former, making use of Eq. 1.6. This, of course, holds only if angular dispersion is indeed negligible.

Howard (Howard, 1987; Howard et al., 1988) has performed a systematic investigation of hydrodynamic dispersion in the CAC. The experimental study was performed in a $12 \mathrm{in.} \mathrm{OD} \mathrm{CAC} \mathrm{unit} \mathrm{at} \mathrm{ORNL} \mathrm{using} \mathrm{Dowex} \mathrm{50W-X8} \mathrm{resin}$ with particle sizes of 37 to $55 \mu \mathrm{m}$ as the chromatographic medium. The annulus thickness was $1.25 \mathrm{~cm}$ and the bed length was $60 \mathrm{~cm}$. In these experiments, an aqueous solution of blue dextran 2000 was used as the feed and deionized distilled water as the eluent. Blue dextran with this molecular weight is completely excluded from the resin matrix and can be used as an effective nonadsorbed tracer. To carry out the experiments, Howard provided the CAC with a constant feed of dilute $(0.5$ to $1.0 \mathrm{~g} / \mathrm{L})$ blue dextran solution and monitored the blue dextran exit concentration profile with a spectrophotometer.

Since blue dextran is not sorbed, peak dispersion results entirely from hydrodynamic effects and diffusion external to the particles. In treating the ex- 
perimental concentration profiles, Howard made the assumption that the blue dextran continuity equation for the $\mathrm{CAC}$ can be written as

$$
\epsilon D_{e} \frac{\partial^{2} C}{\partial z^{2}}=\epsilon \frac{\partial C}{\partial t^{\prime}}+u \frac{\partial C}{\partial z},
$$

where $D_{e}$ is an effective dispersion coefficient which incorporates the effects of axial and angular dispersion. This treatment is, of course, not rigorous, but it provides a reasonable quantitative way of comparing dispersion data for the $\mathrm{CAC}$ with dispersion data for conventional beds. A solution of Eq. 1.9 obtained by Hill (1977) for infinitesimal feed pulses applied to a fixed bed was adapted to the CAC via Eq. 1.6 and used to determine $D_{e}$ from a fit of the experimental concentration profiles. The results of Howard's CAC measurements are summarized in Fig. 1.5 in the form of $\epsilon P e=u d_{p} / D_{e}$ vs $R e=u d_{p} / \nu$, where $d_{p}$ is the particle diameter and $\nu$ is the fluid kinematic viscosity. This figure also shows Howard's measurements of axial dispersion for a fixed bed packed with the same resin used in the CAC experiments, and a line calculated from a correlation for axial dispersion in packed beds that is available in the literature (Butt, 1980). As seen in Fig. 1.5, the fixed-bed data compare well with the literature correlation, while the $\mathrm{CAC}$ data, although rather scattered, fall below. On average, the effective Peclet number for the CAC unit is about 0.1, about two times smaller than the value found for fixed beds. Thus, dispersion in the CAC appears to be two times larger than in fixed beds. However, while this may be significant for gas adsorption or high-pressure liquid chromatography (HPLC) operations using very small particles, the effects of increased dispersion on typical preparative or production-scale liquid chromatographic operations are quite small. These processes tend to be dominated by film and intraparticle mass transfer resis- 
tances which largely determine the spreading of the chromatographic peaks. As an example, Fig. 1.6 (Howard, 1987) shows CAC concentration profiles calculated for conditions corresponding to the separation of glucose-fructose mixtures (a complete description of this separation is given in Sect. 2). The analytical solution shown here neglected dispersion (i.e., $D_{e}=0$ ), while the numerical solution obtained by collocation incorporated the effects of $D_{e}$. The results of these calculations are quite close and can be generalized to show that dispersion is only important in the CAC if mass transfer resistances are small. Otherwise, as is common in liquid chromatographic operation, the effects of increased hydrodynamic dispersion can be neglected for solutes which are retained by the sorbent.

\subsection{SCALEUP OF THE CAC}

Perhaps the most critical scaleup issue in CAC technology is the effect of increased annulus thickness. While the majority of past experiments were conducted with a narrow annulus, a significant number of experiments were performed with packed annuli ranging from $1 \%$ to about $96 \%$ of the crossection of the outer shell. A summary of the CAC units used at ORNL for these runs is given in Table 1.

The experimental work was performed for the separation of mixtures of copper, nickel, and cobalt with Dowex 50W-X8 ion-exchange resin in the ammonium form. The results are presented in Figs. 1.7, 1.8, and 1.9 in the form of resolution (a measure of the degree of separation) as a function of the ratio of feed and eluent rates. As seen in these figures, neither the annulus thickness nor the size 


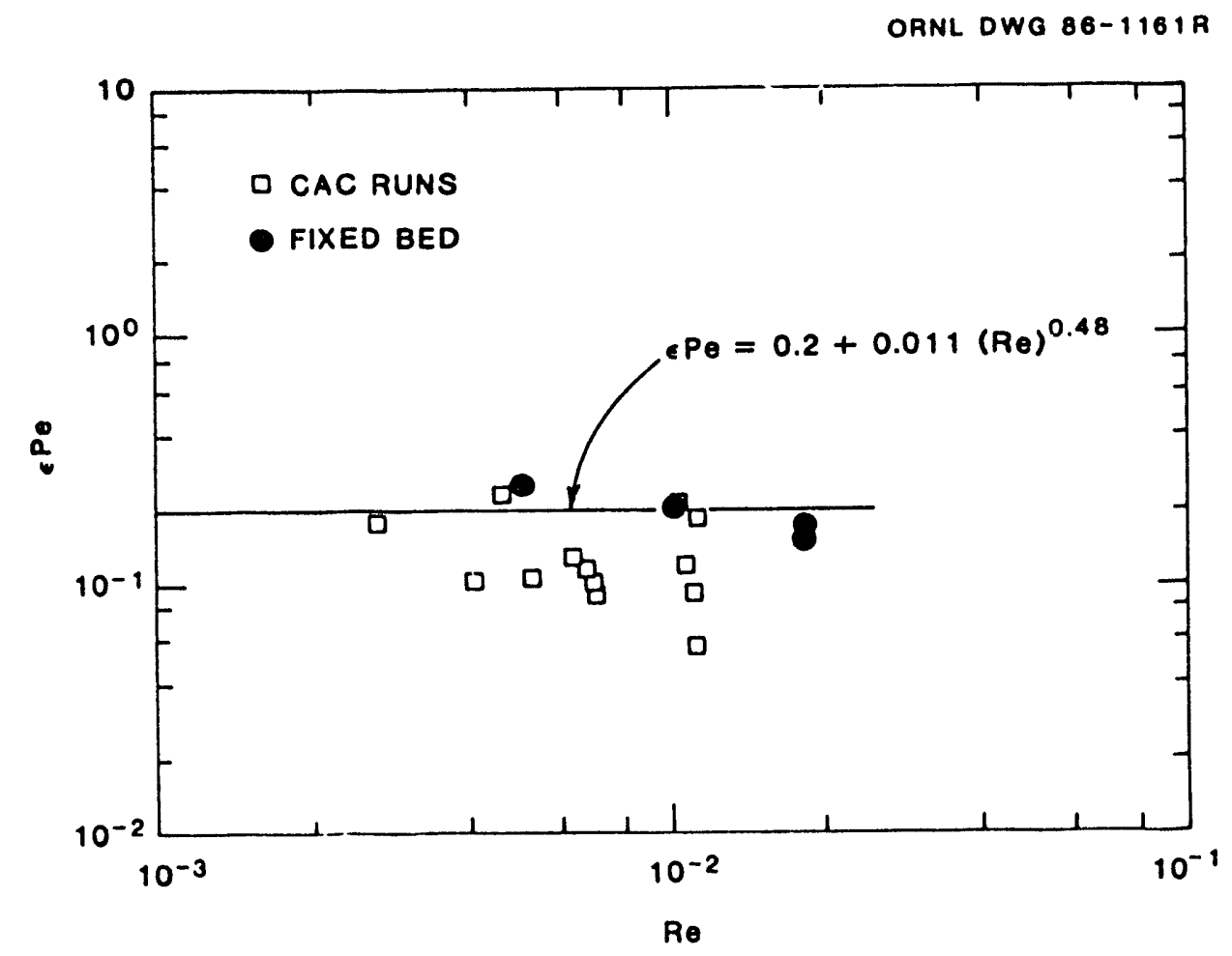

Fig. 1.5 Experimental and calculated dependence of Peclet number on Reynolds number from fixed-bed and CAC experiments (Howard et al., 1987). 


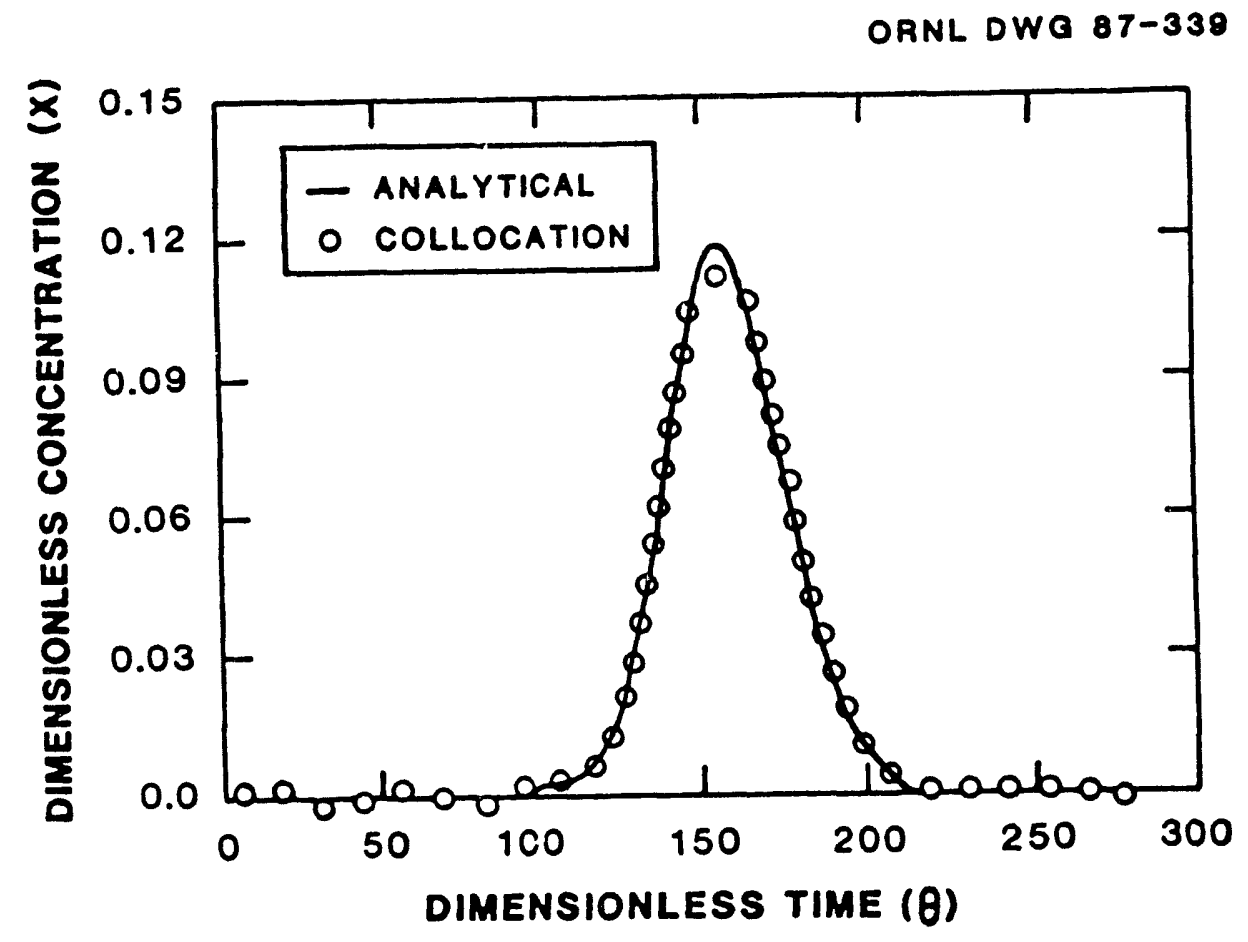

Fig. 1.6 Comparison of the analytical and orthogonal collocation solutions for the CAC (Howard et al., 1987). 
of the CAC appeared to affect the resolution for appropriately scaled conditions. While resolution remained constant, the throughput increased with the annulus thickness.

Obviously, we might expect increased hydrodynamic dispersion as the annulus thickness is increased. Once again, however, provided that mass transfer resistances are signiticant, the effects of increased dispersion are negligible (as indicated by the experimental results).

Other scaleup issues include the operation of the $\mathrm{CAC}$ at high loadings and high feed concentrations. These effects are discussed in Sect. 2 in the context of the industrial separation of sugar mixtures.

\subsection{RESEARCH SCOPE AND APPROACH}

\subsubsection{Research Scope}

While elution and displacement chromatographic techniques are well established in conventional fixed-bed chromatography, the principles and applications of $\mathrm{CAC}$ have been investigated mainly for isocratic operations. Previous authors have demonstrated that the CAC can separate multicomponent mixtures in a truly continuous manner. With a few exceptions, however, the majority of the systems studied thus far have been separated by the method of isocratic elution. Possible advantages of using stepwise elution and displacement chromatographic methods on the CAC have not been extensively investigated and are essential

if Continuous Annular Chromatography is to become an important industrial process. In this work, improved operating methods were investigated and novel applications were explored in the food, hydrometallurgical, and biochemical in- 
Table 1. Physical characteristics of the $\mathrm{CAC}^{a}$ units used at ORNL

\begin{tabular}{cccccc}
\hline & $\begin{array}{c}\text { Annulus } \\
\text { width, } \Delta r \\
(\mathrm{~mm})\end{array}$ & $\begin{array}{c}\text { Outer annulus } \\
\text { radius, } \\
r_{0}(\mathrm{~mm})\end{array}$ & $\Delta r / r_{0}$ & $\begin{array}{c}\text { Annular bed } \\
\text { 'x'-sectional } \\
\text { area }\left(\mathrm{cm}^{2}\right)\end{array}$ & $\begin{array}{c}\% \\
\text { Available } \\
\text { area }^{c}\end{array}$ \\
\hline CAC-ME & 6.4 & 44.5 & 0.14 & 16.5 & 26.5 \\
-2 & 12.7 & & 0.29 & 30.4 & 48.9 \\
-3 & 22.2 & & 0.50 & 46.6 & 74.9 \\
-4 & 31.8 & & 0.71 & 57.0 & 91.6 \\
CAC-II & 12.7 & 139.7 & 0.09 & 106.4 & 17.3 \\
-2 & 50.8 & & 0.36 & 364.8 & 59.5 \\
-3 & 124.3 & & 0.82 & 592.0 & 96.7 \\
CAC-III & 31.8 & 222.3 & 0.14 & 411.7 & 26.5 \\
\hline
\end{tabular}

a Continuous annular chromatograph.

${ }^{b}$ From Begovich et al., (1983).

c \% available area is the cross-sectional area of the annular bed divided by the maximum possible area, bases on $r_{0}$. 


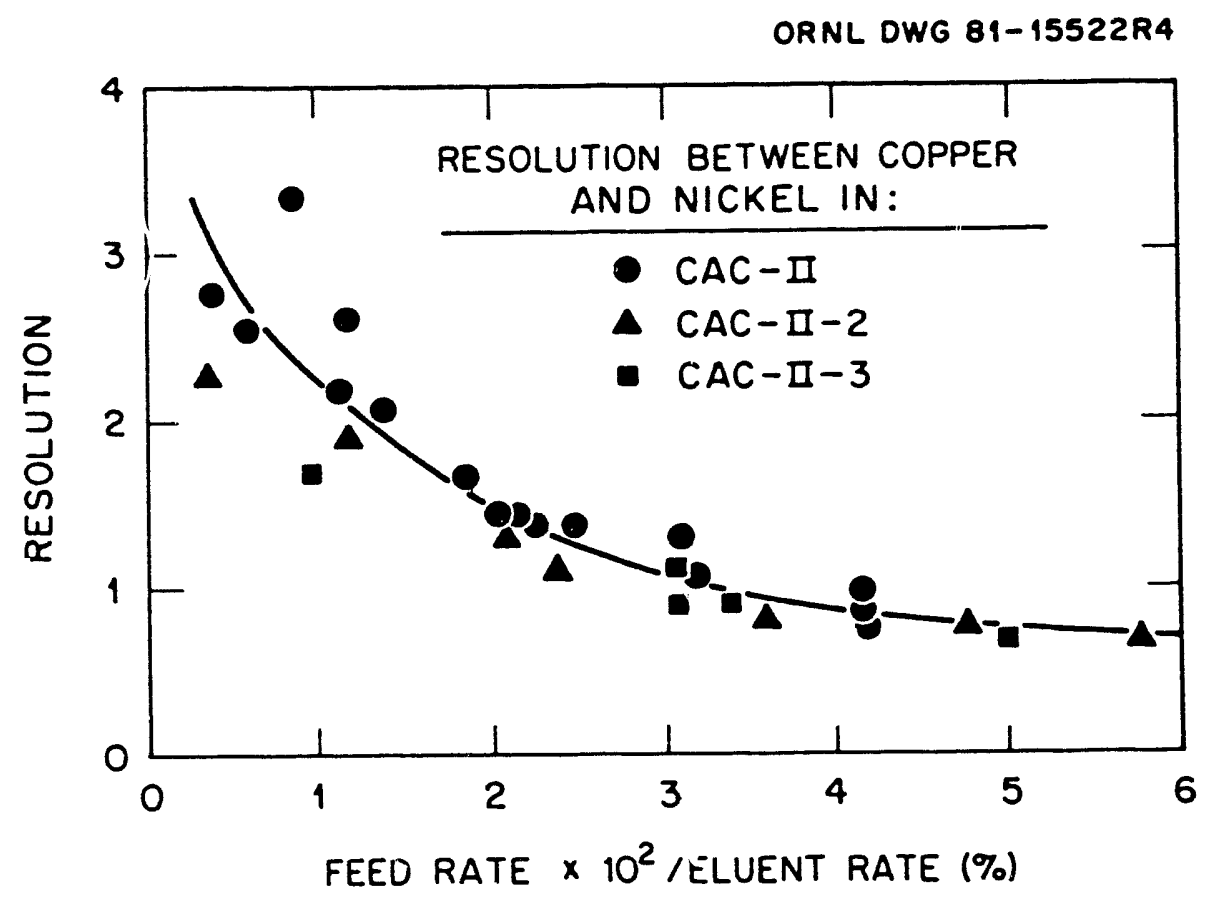

Fig. 1.7 Resolution between copper and nickel as a function of feed percentage in the CAC-II with the different annulus widths given in Table 1 (Begovich et al., 1983). 


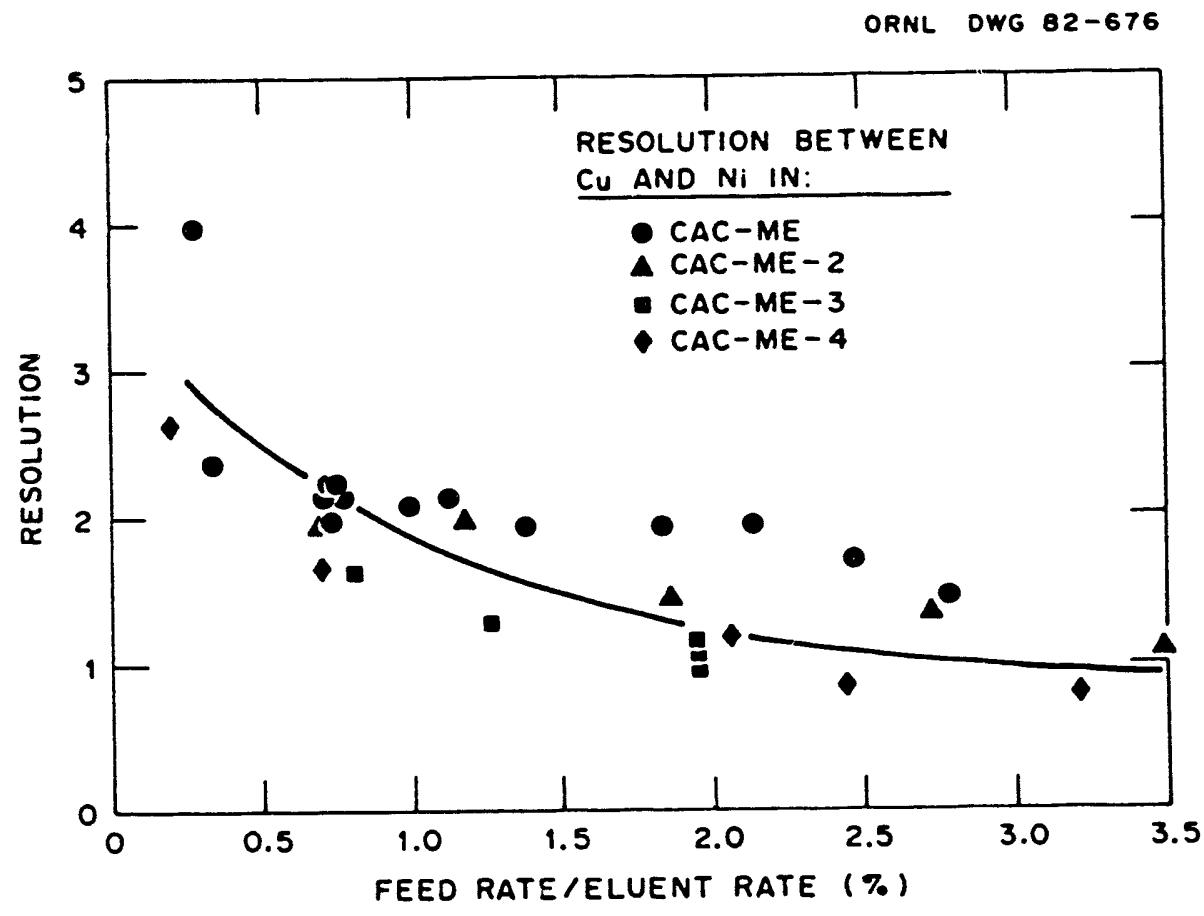

Fig. 1.8 Resolution between copper and nickel as a function of feed percentage in the CAC-ME with the different annulus widths given in Table 1 (Begovich et al., 1983). 
ORNL DWG $82-670$

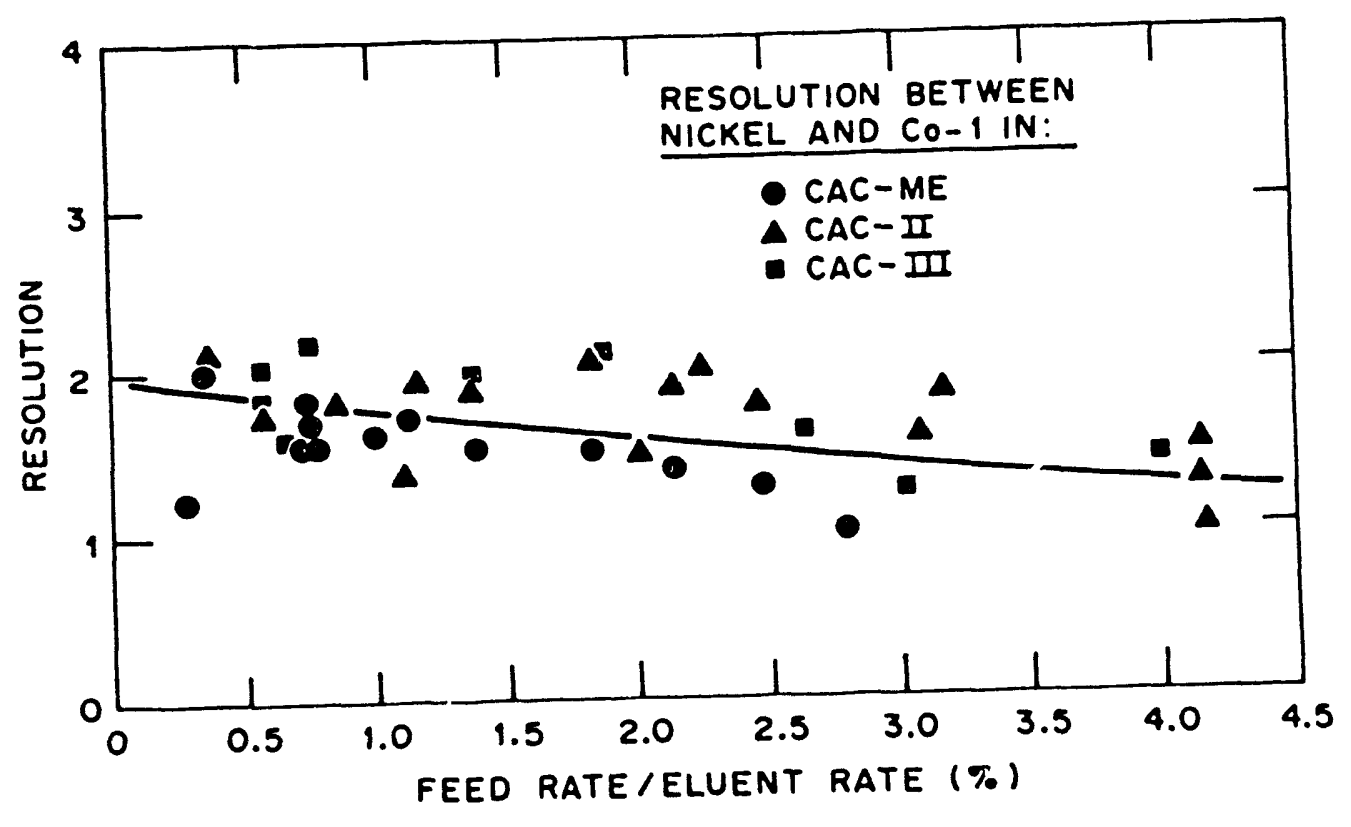

Fig. 1.9 Resolution between nickel and cobalt as a function of feed percentage in three different CAC units. The dimensions of the CAC-ME, the CAC-II, and the CAC-III are given in Table 1 (Begovich et al.,1982). 
dustries. The following systems were studied:

1. The separation of synthetic and industrial high-concentration (up to $60 \%$ by weight) glucose-fructose mixtures was studied as an example of the application of $\mathrm{CAC}$ to an industrial separation. Currently, these mixtures are separated in large, fixed columns with the calcium form of a cation-exchange resin. A similar adsorbent was used here. Since these sugars exhibit linear equilibrium, gradient elution and displacement techniques were not applicable and the CAC was operated in the isocratic mode. The objectives of these experiments were to test the limits of operation of the CAC in terms of high loading and high feed concentrations in order to identify and propose solutions to problems related to industrial scaleup.

2. The separation of dilute mixtures of iron $\left(F e^{3+}\right)$ and chromium $\left(\mathrm{Cr}^{3+}\right)$ ions was studied using the ammonium form of a cation- exchange resin and stepwise elution operation. While this technique has already been demonstrated in a few preliminary experiments at ORNL, this is the first study that determines and quantifies the factors affecting optimization of the separation performance. This system was chosen for ease of operation and for its ability to simulate low metal-ion solutions that are often found in hydrometallurgy.

3. The separation of multicomponent mixtures of amino acids (glutamic acid, valine, and leucine) by ion exchange was investigated as an example of displacement development chromatography. This was the first study to apply this technique to the CAC. Amino acids were used because they are currently separated by fixed-bed displacement development and they represent an im- 
portant potential industrial application of the process.

Relevant literature and background material on each application will be covered in the discussion of each individual study.

\subsubsection{Research Approach}

The purpose of this study was to develop three different modes of chromatography using the $\mathrm{CAC:}(1)$ isocratic elution (single fluid) of high-concentration mixtures, (2) stepwise elution, and (3) displacement development. The three systems studied (sugars, metal ions, and amino acids) behaved differently with the sorbent and allowed the three different modes of operation. The desired capability was to understand factors that affect each system, as well as realize which mode of operation is more appropriate for industrial applications.

\subsubsection{Isocratic Elution: Separation of Sugars}

Successful separation of low-concentration mixtures of glucose and fructose on the CAC has been performed by Howard et al. (1988). The equilibrium isotherms for the system have been defined, and low-concentration, low-loading experiments that vary different process parameters have been performed on a bench-scale CAC. This information was utilized for the scaleup to a pilot-scale unit. The following approach was taken:

1. Experiments were conducted with a fixed column at both low and high concentrations using a synthetic mixture. 
2. CAC experiments were conducted with a low-concentration synthetic glucosefructose mixture to verify that scaleup to the pilot-scale CAC performed as predicted by the idealized mathematical model developed by Howard.

3. CAC experiments were then performed with industrial sugar mixtures for conditions involving:

(a) low concentration - low loading,

(b) low concentration - high loading, and

(c) high concentration - high loading.

Experiments set up in this way allowed the determination of concentration and loading effects on the pilot-scale CAC unit.

4. Finally, fixed-bed, CAC, and theoretical predictions were compared where applicable. Since one goal was to be able to predict CAC effluents from fixedbed data, equilibrium and mass transfer parameters of the experimental study were obtained from fixed-bed experiments and used in the model predictions of the CAC performance. Analysis of these results should allow identification of problems unique to CAC scaleup.

\subsubsection{Stepwise Elution: Separation of Metal Ions}

Metal-ion separations by ion exchange using both isocratic and gradient elution are well documented in fixed-bed chromatographic separations. As discussed earlier, stepwise and gradient elution techniques have some advantages over isocratic elution, such as decreased dilution and increased bed capacity. 
A few preliminary experiments on stepwise elution have been performed by Canon and Sisson (1978) and Begovich and Sisson (1980) for dilute metal-ion systems. These preliminary experiments indicated that there might be a significant process improvement using CAC in the stepwise elution mode. Therefore, a metal-ion system consisting of mixtures of iron and chromium was used to demonstrate and quantify these advantages. The following approach was taken:

1. Isocratic chromatography experiments were carried out in a fixed-bed system to determine equilibrium and mass transfer parameters of the separation system. These data were used in a preliminary design of CAC experiments.

2. Preliminary isocratic elution experiments were performed on the bench-scale $\mathrm{CAC}$ to gain insight into the proper operating parameters for successful stepelution runs. These experiments could then be compared to later CAC stepwise eluent runs in order to quantify the improved performance.

3. Stepwise elution experiments were made which varied CAC parameters such as rotation rate, feed and eluent concentrations, and step eluent position. This information was then used to design a CAC experiment that demonstrated the major improvements of using step elution.

4. Finally, a mathematical model was developed to describe and optimize stepwise elution operation based on the independently determined equilibrium and mass transfer parameters.

\subsubsection{Displacement Development: Separation of Amino Acids}


Displacement development is a mode of chromatography which offers the advantage of allowing simultaneous separation and concentration of multicomponent mixtures. Industrially, amino acid separations are performed using displacement chromatography.

Displacement chromatography had not been previously performed on the CAC, so it was necessary to modify the existing equipment for displacement separations. The following approach was taken:

1. Equilibrium isotherms for the uptake of different amino acids by Dowex $50 \mathrm{~W}$ $\mathrm{X} 8$ ion-exchange resin were determined in batch experiments.

2. Fixed-bed separations of a selected amino acid system were then carried out to identify appropriate operating conditions to be used in CAC experiments.

3. The CAC was modified for displacement development operation and the equipment set up experimentally verified. CAC experiments were performed which varied operating parameters such as rotation rate, displacer concentration, and feed concentration.

4. Finally, a mathematical model was developed and compared to experimental results for both fixed-bed and $\mathrm{CAC}$ experiments.

The results of these studies are shown separately in Sects. 2, 3, and 4 for the three experimental systems and methods of operation. Individual conclusions are drawn in each chapter for the corresponding system. Then, overall conclusions and recommendations are given in Sect. 5 . 


\section{SEPARATION OF SUGARS BY ISOCRATIC ELUTION}

\subsection{INTRODUCTION}

Liquid chromatography is being used today for the production-scale separation of glucose and fructose, which are important products in the food processing industry. Glucose is used as a fermentable sugar in the baking industry, as a feedstock for biochemical synthesis (such as low-calorie beer production), and as a sweetener in some beverage formulations. It is also used both as a sweetener and as a control on softness and crystallization in the confectionery industry (Bollenback, 1983).

Before the 1980s fructose was primarily used mixed with glucose and sucrose. The development of technologies in the 1970s for producing fructose in an isomerized mixture improved the availability of fructose, but even in 1981 the price of fructose was so high that it was not competitive with sucrose and corn syrups as a commercial sweetener. Crystalline fructose, which is 1.3 to 1.8 times sweeter than sucrose, is used as a sweetener of dietary foods because less fructose is required to provide the same sweetness; hence, less calories. Today, it is commercially used in the production of jams, jellies, preserves, cake mixes, puddings, gelatin desserts, ice cream, and candies (Bollenback, 1983).

The most common type of fructose produced, high-fructose corn syrup (HFCS), contains approximately $42 \%$ fructose, $52 \%$ glucose, and $6 \%$ oligosaccharides on a dry-weight basis. Commercial products typically contain $42 \%$, $55 \%$, or $90 \%$ fructose. Recently, however, there has been an increased demand for both purer fructose syrup (90\% fructose) and crystalline fructose ( $>99 \%$ fructose). To meet this demand, a variety of chromatographic processes have been developed to purify HFCS. These processes most commonly involve the use of a sulfonated, polystyrene ion-exchange resin in the calcium form. This material is used, for example, by Hoffman-La Roche at their plant in Thomson, Illinois (Hebeda, 1983).

This project investigated the separation of industrial grade sugars on the CAC 
as a first step toward evaluating its possible incorporation into the industrial process.

\subsection{SUGAR SEPARATIONS WITH ION-EXCHANGE RESINS}

\subsubsection{Solution Chemistry}

Crystalline D-glucose can exist as three different forms, $\alpha$-D-glucose, $\alpha$-Dglucose hydrate, and $\beta-D$-glucose. When $\alpha-D$-glucose is dissolved in water,its optical rotation gradually diminishes to an equilibrium value of 52.7. This process, called mutarotation and illustrated in Fig. 2.1, is exhibited by all three forms of crystalline $\mathrm{D}$-glucose, resulting in an equilibrium solution with $62 \%$ in the $\beta$ form and $38 \%$ in the $\alpha$ form (Fig. 2.2) (Eliel, 1962). This equilibrium value doess not significantly change over a wide range of temperatures and concentrations. In solution, $\alpha$-D-giucose and $\beta$-D-glucose have cyclic ring conformations called pyranoses and are in the $\mathrm{C} 1$ chair form.

Crystalline D-fructose exists as $\beta$-D-fructopyranose and is a stereoisomer of glucose. In solution, the crystalline form undergoes mutarotation to a mixture of $\alpha$ - and $\beta$-D-fructopyranose and $\alpha$ - and $\beta$-D-fructofuranose. Haworth projections of these four structures are illustrated in Fig. 2.3. At $20^{\circ} \mathrm{C}, 80 \mathrm{wt}$ $\%$ is $\beta$-D-fructopyranose, $18 \mathrm{wt} \%$ is $\beta$-D-fructofuranose, and 2 wt $\%$ is $\alpha$-Dfructofuranose, with trace amounts in the $\alpha$-D-fructopyranose form. As the temperature increases, the relative percentage of $\beta$-D-fructopyranose decreases, while the percentages of $\beta$-D-fructofuranose and $\alpha$-D-fructofuranose increase (Doddrell and Allerhand, 1971; Shallenberger, 1973). At lower temperatures, fructose is sweeter because more is in the sweeter fructofuranose form (Bollenback, 1983). Unlike glucose, fructose undergoes complex mutarotation consisting of an initial fast step followed by a slow step. Interconversion between the furanose and pyranose forms is fast, while anomerization between the $\alpha$ and $\beta$ forms is slow (Eliel et al., 1962; Gronlund and Andersen, 1966; Isbell and Pigman, 


$$
\begin{aligned}
& \text { ORNL DWG 87-347 }
\end{aligned}
$$

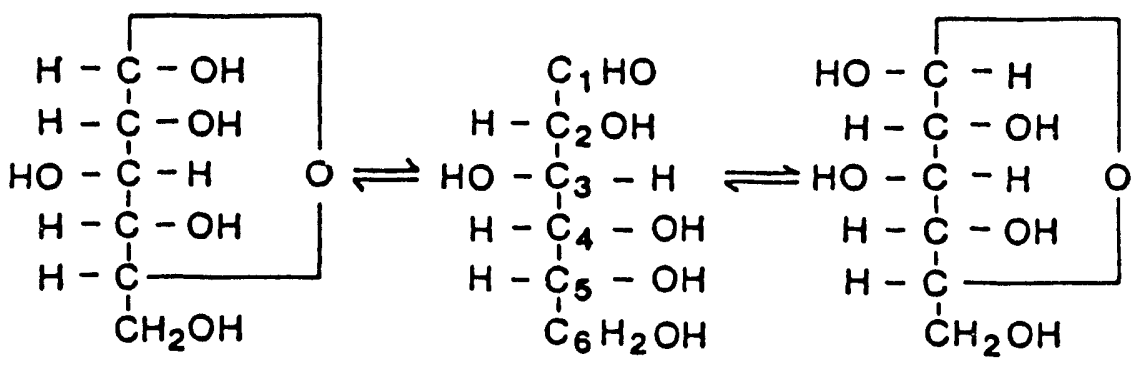

$$
\begin{aligned}
& a-D-G L U C O S E \quad \quad \beta-D-G L U C O S E
\end{aligned}
$$

Fig. 2.1 Mutarotation of $\alpha$ - and $\beta$-D-glucose.

ORNL DWG $87-341$
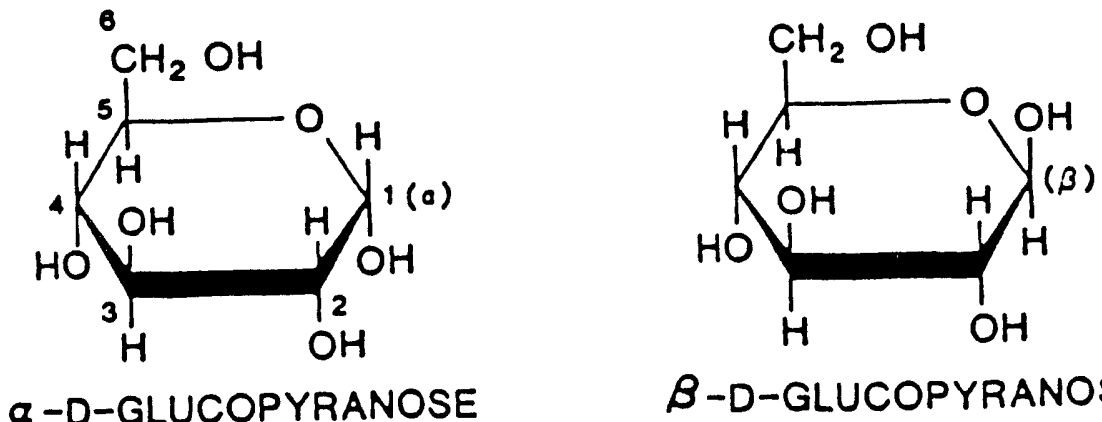

a-D-GLUCOPYRANOSE

$\beta$-D-GLUCOPYRANOSE

Fig. 2.2 Haworth projection formulas of $\alpha$-and $\beta$-D-glucopyranose (Lehninger, 1977). 
1938).

\subsubsection{Chromatographic Separations}

A number of chromatographic media have been investigated for the separation of sugars. The primary focus has been on alkali, metal-exchanged, strong-acid cation-exchange resins and zeolite molecular sieves. Industrially, the most widely used media are strong-acid cation-exchange resins in either the barium or calcium form, with the calcium form being more effective for the glucose-fructose separation (Goulding, 1975). Other factors which need to be considered when selecting an ion-exchange resin are particle size, ionic functionality, degree of cross-linking, and porosity. In this work, the calcium form of Dowex 50W-X8, a strong-acid cation-exchange resin, was used.

The organic matrix of this resin is cross-linked polystyrene divinylbenzene. The resin has an $8 \%$ degree of cross-linking and sulfonic acid functional groups. The optimum range of cross-linking for the glucose-fructose separation is believed to be 4 to $8 \%$ (Welstein and Sauer, 1984). The degree of cross-linking of a resin determines its moisture content and, ultimately, its mass transfer and diffusional characteristics. If the resin has a high moisture content or a low degree of cross-linking, monosaccharides may more easily diffuse in and out of the resin bead. However, the total volume capacity is reduced so there is less calcium for complexation and, hence, a reduced separating capacity. Dowex 50W, with an $8 \%$ degree of cross-linking, was chosen as a compromise between stiffness and selectivity. This resin has a total ion-exchange capacity of $1.7 \mathrm{meq} / \mathrm{ml}$, and is thermally stable up to $150^{\circ} \mathrm{C}$. The resin can tolerate extremes in $\mathrm{pH}$ and is compatible with many alcohol and hydrocarbon solvents. It is sufficiently stiff to resist excess swelling and is sufficiently stable to withstand periodic defouling with strong chemical agents. Furthermore, it is commercially available in a variety of particle sizes, its ionic form can be interchanged easily, and it has the low cost required for a large-scale operation. 
ORNL DWG 89A-751
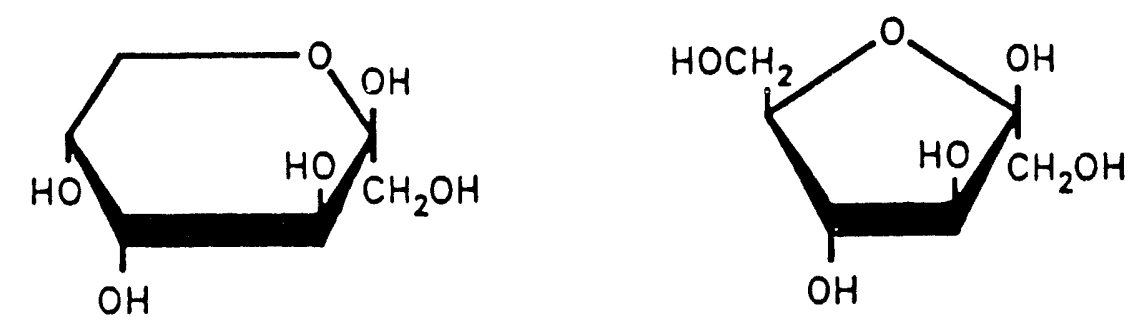

$\beta$-D-FRUCTOPYRANOSE

$\beta$-D-FRUCTOFURANOSE
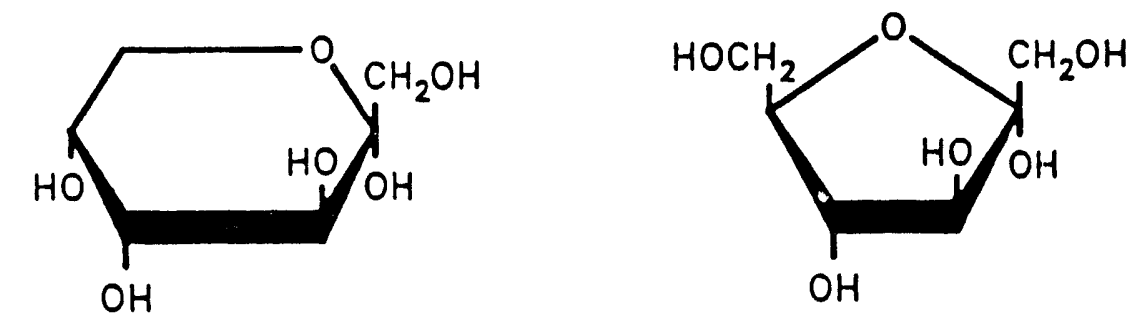

$\alpha$-D-FRUCTOPYRANOSE

$\alpha-D-F R U C T O F U R A N O S E$

Fig. 2.3 Haworth projection formulas of $\alpha$ - and $\beta$-D-fructopyranose and fructofuranose (Lehninger, 1977). 
The separation of a glucose-fructose mixture on this strong cation-exchange resin is achieved by ligand exchange. In this application, a ligand is used to define a molecule or ion attached by a coordinate link to a metal ion to form a complex ion. Glucose and fructose are polyols, molecules which contain numerous hydroxyl groups. In this case, ligand exchange occurs when the hydroxyl groups of water molecules, which are held in a coordination complex with the calcium ions, are exchanged with the hydroxyl groups of glucose and fructose. The strength of this complex increases as the availability for a hydroxyl-group metal-ion complex increases. For glucose and fructose, two complex-forming hydroxyl groups must be located at or near a particular optimum distance from each other for calcium complexation to occur. According to Reeves (1949), this orientation occurs when hydrox'' groups on adjacent carbon atoms are oriented in the true cis position ( $0^{\circ}$ or $\pm 60^{\circ}$ projected angle) or in the trans chair form. Nuclear magnetic resonance spectroscopy and electrophoresis studies indicate that in pyranose sugars the sequence $a x-e q$ of hydroxyl groups can form weak bidentate chelates with suitable cations such as calcium (Goulding, 1975). When two bidentate chelating sites are present, the number of molecules complexed to the cation doubles. It has been shown that $\beta$-D-fructopyranose has two $a x-e q$ groupings, $\alpha$-D-fructopyranose has one $a x-e q$ grouping, $\alpha$-D-glucopyranose has one $a x-e q$ grouping, and $\beta$-D-glucopyranose has no $a x-e q$ groupings. For fructose, the exocyciic hydroxyl group in $\alpha$ - and $\beta$-fructopyranose forms might also contribute to the strength of the complex. Therefore, the conformation in space of the hydroxyl groups in pyranose sugars determines the strength of the coordination complex and the value of the distribution coefficient (Waltonand Navratil, 1981; Welstein and Sauer, 1984). The conformational structures of glucopyranose and fructopyranose are illustrated in Fig. 2.4.

Furanose complexing was not investigated because it is believed that pyranosefuranose interconversion can occur only if the resulting metal-ion complex is more stable (Goulding, 1975). In addition, since mutarotation is fast through interconversion and since a strong metal-ion complex is formed with the pyranose 
ORNL DWG 89A-752

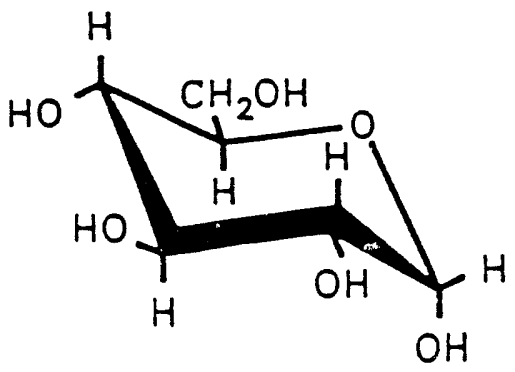

$\alpha-D-G L U C O P Y R A N O S E$

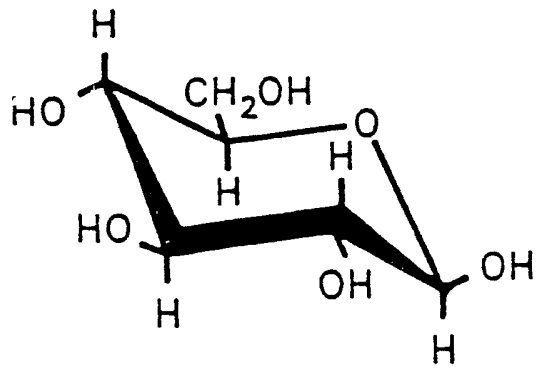

$\beta-D-G L U I C O P Y R A N O S E$

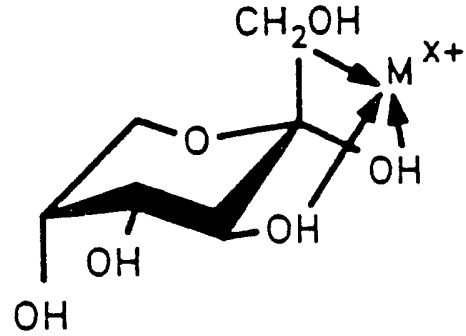

$\alpha-D-F R U C T O P Y R A N O S E$

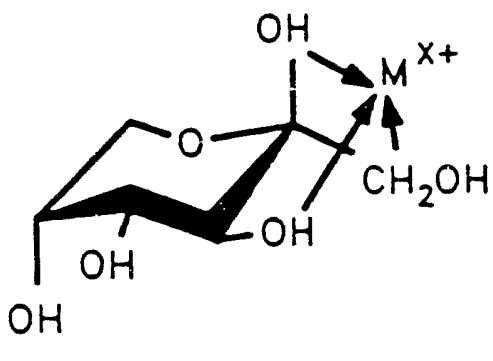

$\beta-D-F R U C T O P Y R A N O S E$

Fig. 2.4 Conformational formula of $\alpha$ - and $\beta$-D-glucopyranose and $\alpha$ - and $\beta$-D-fructopyranose (Lehninger, 1977; Goulding, 1975). 
forms, the resin may be thought of as a "sink" for the fructopyranose forms. If one considers the resin a sink for the pyranose forms, Le Chatelier's principle predicts an equilibrium shift toward the production of fructopyranose. Thus, a glucose-fructose solution containing metal ions available for complexing will consist of glucose in the $\alpha$ - and $\beta$-pyranose forms and fructose predominantly in the $\beta$-pyranose form.

A multicomponent solution containing glucose, fructose, and oligosaccharides, the components in a typical HFCS, can be separated on a strong cation-exchange resin in the calcium form. In practice, the oligosaccharides elute first since they are excluded from the resin matrix due to their size. Next, $\beta$-D-glucose (no $a x-e q$ groups) elutes, followed by $\alpha$-D-glucose (one $a x-e q$ group), and, finally, $\beta$-D-fructose (two $a x-e q$ groups). The resolution between $\alpha$ - and $\beta$-D-glucose forms is slight and often results in a single irregular peak with $\alpha$-D-glucose forming a "hump" on the tail of the $\beta$-D-glucose peak.

\subsection{INDUSTRIAL SUGARS: PRODUCTION AND SEPARATION}

The production of HFCS from corn starch is extensive and involves about 18 separate steps in 5 principal operations. The following operations are typically performed: (1) liquefaction, (2) saccharification, (3) sample preparation for isomerization, (4) isomerization, and (5) separation and purification (Byers and Holmes, 1988). Only the separation and purification steps will be reviewed as they pertain to this work.

The primary function of the separation step is to concentrate the product of the isomerization step. The maximum fructose concentration available at equilibrium from the isomerization step is 50-55 wt \% on a dry-weight basis. However, residence time in an isomerization column is adjusted to produce a syrup of 42 to $45 \mathrm{wt} \%$. This is the only product of a typical fructose plant unless a separation system is included to produce a purified product. Purified products, typically containing more than $85 \%$ fructose, can then be blended 
with the isomerization reactor stream containing 42 to $45 \%$ fructose to produce a $55 \%$ HFCS. Fifty-five percent fructose is used in soft drink manufacturing and has replaced sugar almost completely in this application. Production of $90 \%$ pure fructose can also be achieved from the separation system, but only with a subsequent decrease in the amount of fructose available for blending. Therefore,

if an existing plant wishes to increase production of enriched fructose (>90\%), it must either decrease its production of the $55 \%$ product or expand its operations. This $90 \%$ stream is often used as the feed for crystallizers and evaporators to produce crystalline (99+\% pure) fructose (Hebeda, 1983; Byers and Holmes, 1988).

\subsection{EXPERIMENTAL METHODS}

\subsubsection{Equilibrium and Fixed Bed Studies}

Synthetic glucose-fructose-sucrose mixtures were prepared as aqueous solutions using analytical grade sugars obtained from the Sigma Chemical Company. The industrial HFCS feedstock was donated by A. E. Staley in Loudon County, Tennessee. HPLC analysis of this feedstock determined that it contained 325 $\mathrm{g} / \mathrm{L}$ glucose, $355 \mathrm{~g} / \mathrm{L}$ fructose, and $26 \mathrm{~g} / \mathrm{L}$ oligosaccharides, or a total of 706 $\mathrm{g} / \mathrm{L}$ sugar. HFCS was used in the pure form or diluted with deionized distilled water. In addition, the kinematic viscosity, $\nu$, of the industrial sugar solutions was measured with a capillary viscometer. The density, $\rho$, was measured with a hydrometer. From the density and kinematic viscosity, the viscosity, $\mu$, was calculated from $\mu=\rho \nu$.

The equilibrium uptake of glucose and fructose by the calcium form of Dowex $50 \mathrm{~W}-\mathrm{X} 8$ has been investigated by Howard et al. (1987) and shown to be essentially linear over the range 0 to $50 \mathrm{~g} / \mathrm{l}$. Ching et al. (1987) have also found linear equilibrium up to $250 \mathrm{~g} / 1$ using similar cation-exchange resins (Duolite) in the calcium form. Ghim and Chang (1982) also used Dowex resins and determined 
the distribution coefficients for glucose and fructose to be 0.23 and 0.60 , respectively. Most investigators have found glucose and fructose to exhibit linear equilibrium, but some have not. Barker et al. (1986) and Ganetsos (1987) found a slight upward curvature of the isotherm at high sugar concentrations. These measurements were extracted over a variety of operating conditions directly from the operation of a process termed "the semicontinuous countercurrent chromatographic refiner" (SCCR). The effects that concentration, temperature, and flow rate have on equilibrium were investigated by these authors. However, these results, which show a nonlinear equilibrium, are somewhat suspect because they would indicate that equilibrium is dependent on flow rate. Generally, it is believed that the isotherms are linear over a wide range of concentrations and become slightly nonlinear at very high concentrations. However, the mathematical advantages of assuming linear behavior are so beneficial and the approximation so good that most investigators have used it in modelling.

Fixed-bed experiments were performed in the apparatus shown in Fig. 2.5. The apparatus consists of a column of stainless steel tubing $0.95-\mathrm{cm}-\mathrm{ID}$ and $107-$ $\mathrm{cm}$ long. Injections were made through a Reodyne six-port valve with a $0.5-\mathrm{cm}^{3}$ sample loop. Dowex 50W-X8 resin (50 $\mu \mathrm{m}$ in diameter) in the calcium form was used as the sorbent. The eluent (deionized distilled water) was pumped through the column by a Milton Roy Constametric IIIG double-cam metering pump. The effluent was continuously monitored with a Waters Model 401 refractive index detector and samples were collected with a fraction collector. These fractions were then analyzed by HPLC utilizing a Biorad Aminex HPX-87C column at $85^{\circ} \mathrm{C}$ (Bio-Rad Catalogue, 1987).

Up to this point, little has been said about the operating temperature. In industrial plants the glucose-fructose separation on fixed-bed columns is performed at 60 to $85^{\circ} \mathrm{C}$. In theory, an elevated temperature should decrease peak tailing and increase mass transfer rates. The real benefit, however, is a decrease in the solution viscosity which greatly effects a concomitant reduction of the resin selectivity for fructose. 


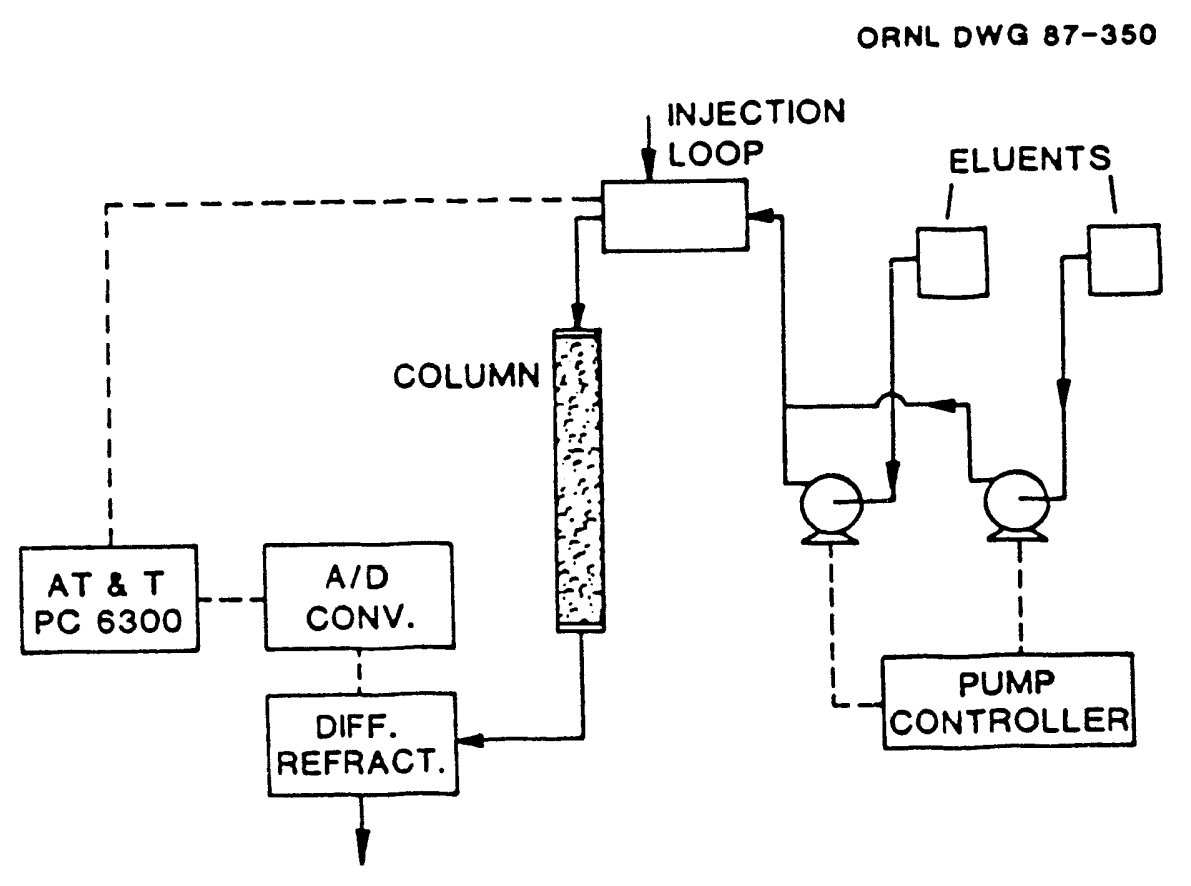

Fig. 2.5 Schematic of the fixed-bed apparatus. 
In this work, the separations were performed at room temperature for practical considerations. First, it is important to determine whether the separation of a glucose-fructose mixture must be done at an elevated temperature. Substantial energy savings could be gained at ambient operating conditions if high-temperature operation is not required. Second, the pilot-scale CAC is not equipped for operation above room temperature and significant modifications would be required. Finally, operating at room temperature poses a more stringent test of the scaleup issues in view of the high viscosity. Therefore, both fixed-bed and pilot-scale experiments were performed at $25 \pm 3^{\circ} \mathrm{C}$.

\subsubsection{Continuous Annular Chromatographic (CAC) Studies}

A schematic of the CAC used in this study, the CAC-ME, is shown in Fig. 2.6 and a photograph of the entire apparatus is shown in Fig. 2.7. The CAC-ME is constructed of Carpenter 20 steel and can withstand pressures up to $1.15 \mathrm{MPa}$. The outside cylinder has an OD of $44.5 \mathrm{~cm}$ and forms a $3.17-\mathrm{cm}$ annulus with the inner cylinder. The bed was packed to a depth of $107 \mathrm{~cm}$, and glass beads with a diameter of $0.018 \mathrm{~cm}$ were packed on top of the resin to a depth of $8.5 \mathrm{~cm}$. The resin is supported by porous, high-density polyethylene plugs located at the top of each of the 120 exit holes. Below each exit hole is a small outlet tube that prevents mixing of different circumferential products. Eluent and feed are introduced through a stationary inlet distributor that extends through the top flange and is sealed by two O-rings. Milroyal Model DC-1-175R positive displacement pumps rated for 1000 psi with flow precision of $\pm 1 \%$ were used to deliver eluent and feed streams. The eluent was allowed to fill the head space above the glass bead layer, while stainless steel nozzles, attached to the stationary distributor, were located within the glass bead layer. This arrangement allows the introduction of feed and eluent into the CAC without significantly mixing the two streams. As the CAC rotates, the giass beads flow around the feed nozzles, which are sufficiently fluid to form a self-repairing layer that prevents them 
from carving a permanent trench within that layer. The fluid velocity remains constant throughout the annulus if the feed and eluent have the same viscosity, and the feed arc of any nozzle is proportional to the ratio of its flow sate divided by the total flow rate. The CAC rotation is controlled by a digitally controlled drive system.

A simple procedure was used to determine the effluent concentration profile. As the $\mathrm{CAC}$ rotates the exit tubes also rotate, while the helical bands that have formed within the annulus remain stationary. Thus, by connecting a single outlet tube to a continuous detector, the detector will receive eluent from all circumferential points as the CAC completes a $360^{\circ}$ turn. If the concentration detection equipment is attached to a timer, an apparently conventional (i.e., concentration vs time) chromatograph is recorded. Angular concentration profiles can then be obtained from this conventional chromatogram by using the transformation $\theta=\omega t$. In this study, a Waters Model R401 refractive index detector with a digitally controlled Constametric pump was used, while a peristaltic pump, set for a flow of $1 / 120$ of the total flow, was used to feed the detector. The outlet from the detector was collected by means of a mechanical fraction collector, and fractions were collected at $4^{\circ}$ intervals along the annulus and saved for HPLC analysis.

\subsection{THEORETICAL DISCUSSION}

Continuity and rate equations for the $\mathrm{CAC}$ are similar to those of a fixed bed. It has previously been shown that the unsteady-state, one-dimensional, continuity equation for a fixed bed is analogous to the two-dimensional, steadystate equation for the CAC by neglecting the angular dispersion term, $D_{0}$, and making the following change of variable:

$$
\theta=\omega t^{\prime}
$$




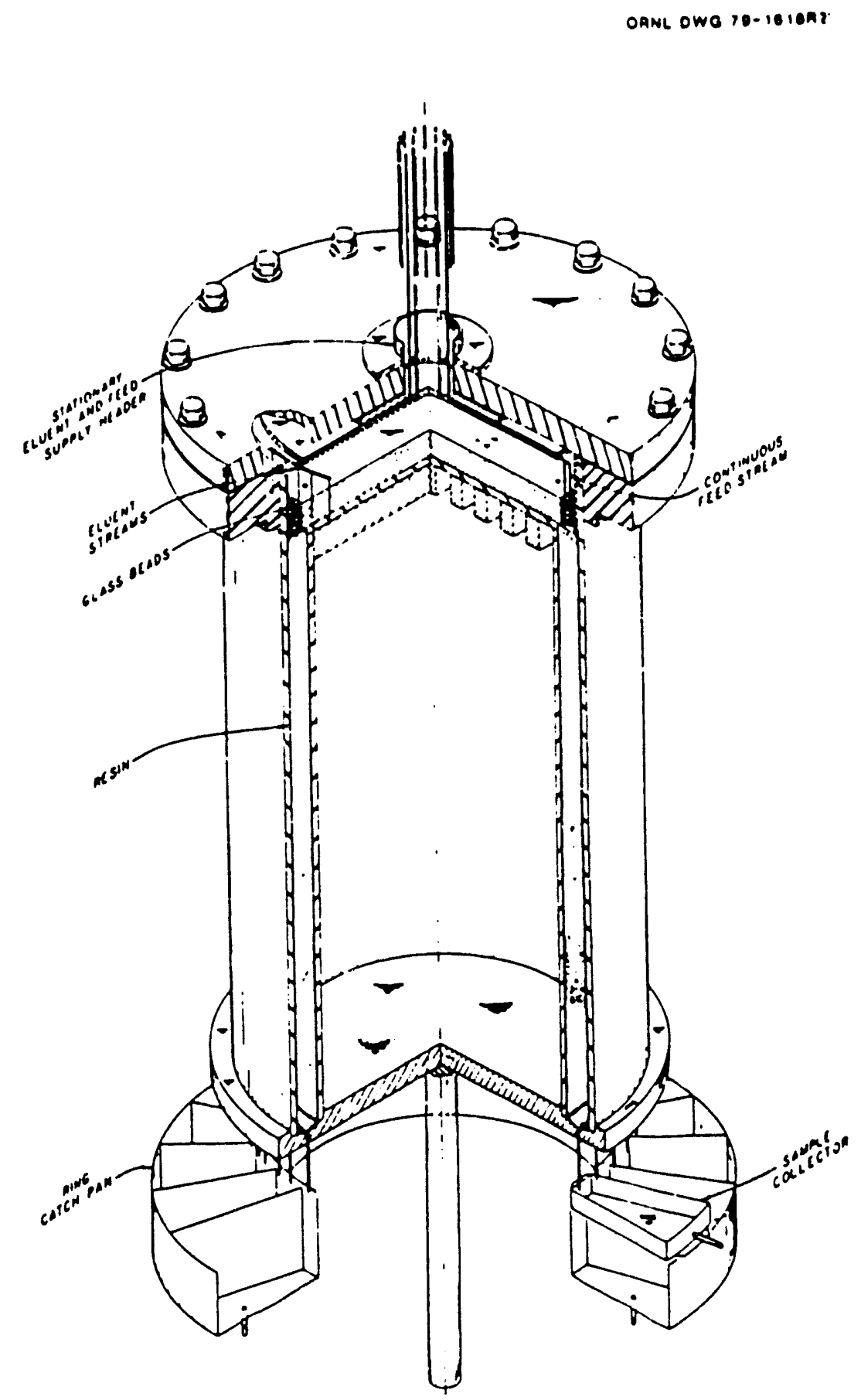

Fig. 2.6 Construction detail of the CAC-ME. The unit has a $445 \mathrm{~mm} \mathrm{OD}$, an annulus width of $31.7 \mathrm{~mm}$, and a usable bed depth of $110 \mathrm{~cm}$. 


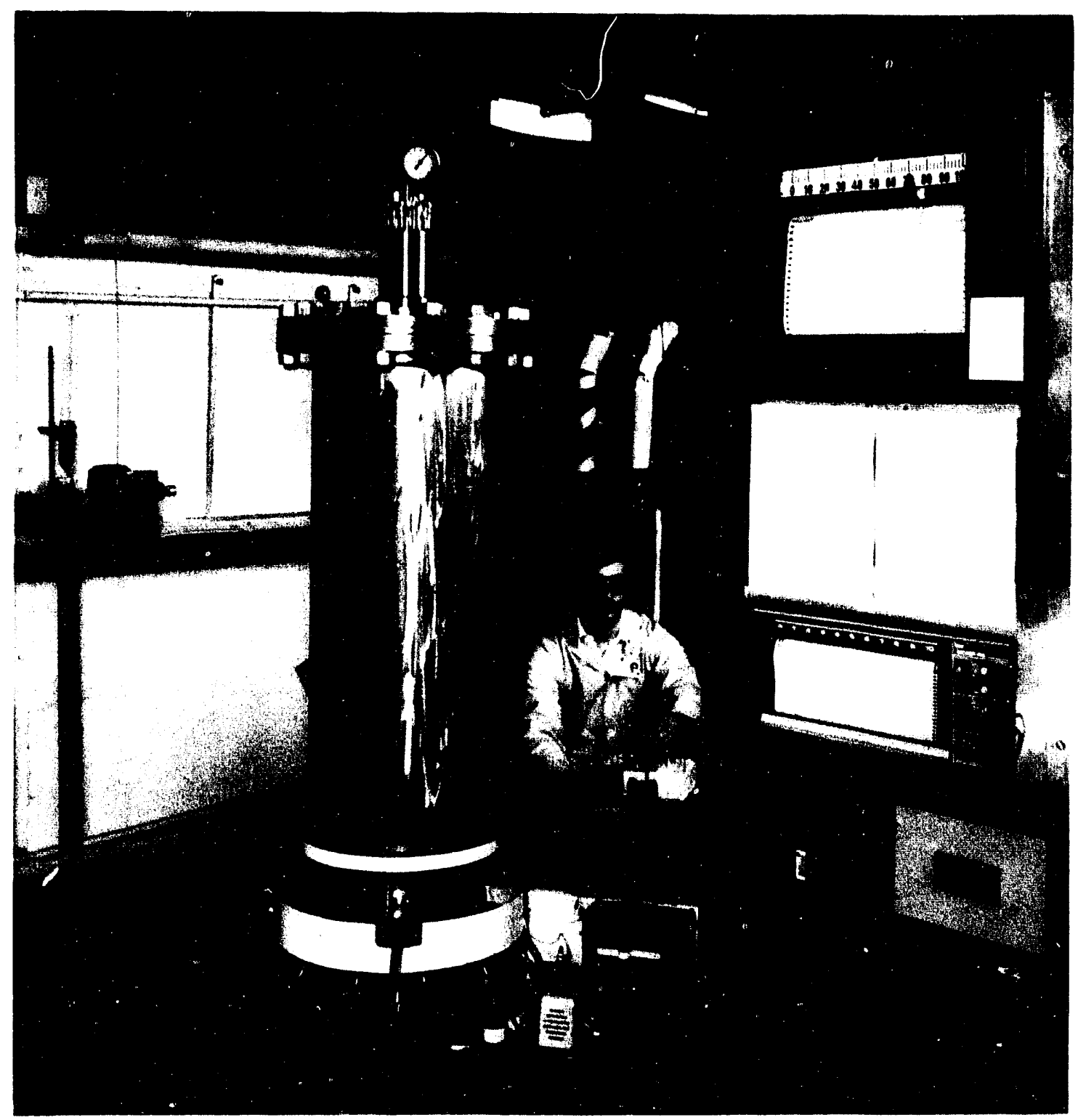

Fig. 2.7 Photograph of the 445-mm pilot-scale CAC showing data acquisition and auxiliary equipment. 
Neglecting axial dispersion, the CAC continuity equation is

$$
\omega \epsilon \frac{\partial C}{\partial \theta}+\omega(1-\epsilon) \frac{\partial q}{\partial \theta}+u \frac{\partial C}{\partial z}=0
$$

A rate equation relates the solid phase concentration, $q$, and the fluid phase concentration, C. A film model was used by Howard (1987) to describe fluidparticle mass transfer and may be written in the transformed coordinate system as

$$
\omega(1-\epsilon) \frac{\partial q}{\partial \theta}=k_{\circ} a\left(C-C^{*}\right)
$$

where $k_{o} a$ is the overall mass transfer coefficient, and $C^{*}$ is the fluid phase concentration in equilibrium with the solid phase (Howard et al., 1988). Boundary conditions for Eqs. 2.2 and 2.3 are

$$
\begin{array}{lll}
\theta=0, & \text { all } z: & q=C=0 \\
z=0, & 0<\theta \leq \theta_{F}, & C=C_{F} \\
& \theta>\theta_{F}, & C=0 .
\end{array}
$$

An analytical solution to these equations for linear equilibrium was found by Thomas (1944) for fixed-bed operations. The Thomas solution can be further simplified if one assumes an infinitely small feed pulse (or feed arc in the case of the $\mathrm{CAC})$, and if the number of transfer units $\left(n=k_{0}, a z / u\right)$ is greater than five. The resulting approximate expression (Sherwood et al., 1975) is

$$
C(z, \hat{t})=\frac{Q}{2 \sqrt{\pi}}\left\{\frac{\left(k_{o} a\right)^{2}}{u^{3} z \hat{t}[(1-\epsilon) K]^{3}}\right\}^{0.25} \exp \left\{-\left[\sqrt{\frac{k_{\circ} a z}{u}}-\sqrt{\frac{k_{o} a \hat{t}}{K(1-\epsilon)}}\right]^{2},\right.
$$

where

$$
\hat{t}=\frac{\theta}{\omega}-\frac{\epsilon z}{u} .
$$


The amount of solute introduced per unit cross-sectional area of the sorbent bed, $Q$, can be calculated from

$$
Q=\frac{C_{F} u Q_{F}}{Q_{T}} \frac{360^{\circ}}{\omega}
$$

where $Q_{F}$ is the feed flow rate and $Q_{T}$ is the total flow rate.

Equation 2.4 is especially useful in determining equilibrium and mass transfer parameters from experimental chromatographic concentration profiles. As shown by Sherwood et al. (1975) the peak maximum will occur when

$$
\sqrt{\frac{k_{o} a z}{u}}-\sqrt{\frac{k_{o} a \hat{t}}{K(1-\epsilon)}}=0
$$

or

$$
\hat{t}_{\mathrm{max}}=\frac{K(1-\epsilon) z}{u}
$$

Thus, the equilibrium distribution coefficient is given by

$$
K=\frac{u}{z(1-\epsilon)} \hat{t}_{\mathrm{max}}=\left(t_{\mathrm{m} a x}-\frac{\epsilon z}{u}\right) \frac{u}{z(1-\epsilon)}
$$

for a fixed-bed experiment, or

$$
K=\left(\frac{\theta_{\mathrm{m} a x}}{\omega}-\frac{\epsilon z}{u}\right) \frac{u}{z(1-\epsilon)}
$$

for a CAC experiment. The same result can, of course, be found from Eq. 1.1. The overall mass transfer coefficient, $k_{o} a$, can be found from the width of the experimental peak at one-half of the maximum peak height, $\Delta$. As shown by Sherwood et al. (1975), from Eq. 2.4 one finds that

$$
k_{o} a=\frac{u}{z} 16(\ln 2)\left(\frac{\hat{t}_{\mathrm{max}}}{\Delta}\right)^{2}
$$

At high feed loadings used in preparative-scale applications, the assumption of an infinitely small feed arc no longer applies and Eq. 2.4 fails. Carta (1988) 
has developed an exact analytic solution for the general case of finite-width, periodic-feed applications while retaining the assumptions of linear equilibrium and negligible axial dispersion. Carta's solution, originally obtained to describe the behavior of a fixed bed, can be transformed with the use of Eq. 2.1 to give the two-dimensional, steady-state solution for the CAC. The resulting expression

$$
\begin{aligned}
\frac{C(z, \theta)}{C_{F}} & =\frac{\theta_{F}}{\theta_{F}+\theta_{E}}+\frac{2}{\pi} \sum_{j=1}^{\infty}\left\{\frac{1}{j} \exp \left[-\frac{j^{2} k_{o} a z}{\left(j^{2}+r^{2}\right) u}\right] \times \sin \left[\frac{j \pi \theta_{F}}{\theta_{F}+\theta_{E}}\right]\right. \\
& \left.\times \cos \left[-\frac{j \pi \theta_{F}}{\theta_{F}+\theta_{E}}+\frac{2 j \pi \theta}{\theta_{F}+\theta_{E}}-\frac{2 j \pi z \omega \epsilon}{u\left(\theta_{F}+\theta_{E}\right)}-\frac{j r k_{o} a z}{\left(j^{2}+r^{2}\right) u}\right]\right\},
\end{aligned}
$$

can then be used to compute concentration profiles where $\theta_{F}$ and $\theta_{E}$ are the feed and elution arcs, and $r$ is given by

$$
r=\frac{k_{o} a\left(\theta_{F}+\theta_{E}\right)}{2 \pi(1-\epsilon) K \omega} .
$$

This equation applies for both large and small feed sectors, or when multiple, evenly spaced feeds are fed to the same column.

The average concentration, $\bar{C}$, between any two angles, $\theta_{1}$ and $\theta_{2}$, can be calculated by integrating Eq. 2.12 resulting in

$$
\begin{aligned}
\frac{\bar{C}(z)}{C_{F}} & =\frac{\theta_{F}}{\theta_{F}+\theta_{E}}+\frac{2\left(\theta_{F}+\theta_{E}\right)}{\pi\left(\theta_{2}-\theta_{1}\right)} \sum_{j=1}^{\infty}\left\{\frac{1}{j^{2}} \exp \left[-\frac{j^{2} k_{o} a z}{\left(j^{2}+r^{2}\right) u}\right]\right. \\
& \times \sin \left[\frac{j \pi \theta_{F}}{\theta_{F}+\theta_{E}}\right] \times \sin \left[\frac{j \pi\left(\theta_{2}-\theta_{1}\right)}{\theta_{F}+\theta_{E}}\right] \\
& \left.\times \cos \left[-\frac{j \pi \theta_{F}}{\theta_{F}+\theta_{E}}+\frac{j \pi\left(\theta_{1}+\theta_{2}\right)}{\theta_{F}+\theta_{E}}-\frac{2 j \pi z \omega \epsilon}{u\left(\theta_{F}+\theta_{E}\right)}-\frac{j r k_{o} a z}{\left(j^{2}+r^{2}\right) u}\right]\right\}
\end{aligned}
$$

The average concentrations can then be used to calculate the purity of the product fraction collected between any two angles $\theta_{1}$ and $\theta_{2}$.

The assumption made in using these equations is linear equilibrium with no axial and angular dispersion. It has already been shown that the glucose-fructose 
system exhibits linear equilibrium at low concentration. Although some nonlinearity has been found at high concentrations, using the analytical solutions can provide valuable insight into the separation process and far outweighs the drawbacks. In practice, some differences between the CAC and fixed-bed experiments may be observed. Factors contributing to these differences include column geometry, dispersion, and flow nonuniformities. Howard et al. (1987) have already investigated dispersion in the $\mathrm{CAC}$ and found it to be roughly twice that in a fixed bed. As discussed in Sect. 1, Howard used a lumped parameter model to account for dispersion in the angular and axial direction, finding that if significant mass transfer resistances are present, the effects of dispersion are negligible. Howard's experiments were performed with low-concentration sugar mixtures. However, because it was expected that flow nonuniformities could be important at high loadings, this work investigated high column loadings with both lowand high-concentration industrial sugar mixtures. This was done to test the limits of "ideal" operation with the purpose of quantifying the conditions for which these nonideal effects become important.

\subsection{RESULTS AND DISCUSSION}

\subsubsection{Equilibrium and Mass Transfer}

In initial experiments, analytical reagent-grade glucose, fruct,ose, and sucrose were used to simulate an industrial corn syrup mixture and to determine equilibrium distribution and overall mass transfer coefficients. These coefficients were found for the pure components in a fixed-bed column using the procedure illustrated in Sect. 2.5. Small loadings and feed concentrations of less than $25 \mathrm{~g} / \mathrm{L}$ were used in these experiments. Since the resolution between $\alpha$-and $\beta$-glucose is only very slight, the distribution coefficient presented will be an average for the two forms. The corresponding mass transfer coefficient for glucose will be considerably larger than its actual value to take into account the apparent peak 
spreading of the $\alpha$ and $\beta$ anomers. Sucrose was used to represent the oligosaccharides found in industrial mixtures because (1) it is larger than each of the two monosaccharides, and (2) it will not form a complex with the sorbent since it contains no $a x-e q$ groupings (Goulding, 1975).

The overall mass transfer resistance includes both fluid- and solid-phast resistance in series and may be written as

$$
k_{o} a=\left(\frac{1}{\frac{1}{k a}+\frac{1}{k^{\prime} K}}\right),
$$

where $k$ is the fluid-phase mass transfer coefficient, $k^{\prime}$ is a solid-phase film mas: transfer coefficient, and $K$ is the distribution coefficient. Previously, Howard et al. (1988) determined that the overall mass transfer coefficient for glucose, fructose, and sucrose on Dowex 50 was dominated by particle size resistance. Therefore, the overall mass transfer coefficient is not a function of flow rate and can be expressed as

$$
k_{o} a=k^{\prime} K
$$

The resulting distribution and mass transfer coefficients, obtained from fixedbed concentration profiles, are given in Taole 2.

A mixture of $25 \mathrm{~g} / \mathrm{L}$ glucose, $25 \mathrm{~g} / \mathrm{L}$ fructose, and $10 \mathrm{~g} / \mathrm{L}$ sucrose was dissolved in the eluent (deionized distilled water) and a pulse experiment was performed ${ }_{i}$. the fixed column described above. A sample volume of $0.5 \mathrm{~cm}^{3}$ and a fic $v$ rate oi $0.96 \mathrm{~cm}^{3} / \mathrm{min}$ were used. Concentration profiles for the various components and the theoretical prediction of these profiles using Eq. 2.4 and the values in Table 2 is illustrated in Fig. 2.8. In general, the model provides a good prediction of the peak time and an approximate prediction of the peak height.

A CAC experiment was made at conditions corresponding to those used for the fixed-bed experiment (the CAC unit and the fixed-bed column have the same length). A feed of $25 \mathrm{~g} / \mathrm{L}$ glucose, $25 \mathrm{~g} / \mathrm{L}$ fructose, $10 \mathrm{~g} / \mathrm{L}$ sucrose, and $1.0 \mathrm{~g} / \mathrm{L}$ blue dextran was used. The blue dextran, obtain from Pharmacia, 
Table 2. Equilibrium and Mass Transfer Parameters for Dowex 50W-X8 $(50 \mu \mathrm{m})$ in the Calcium Form

\begin{tabular}{ccc}
\hline Sugar & $\begin{array}{c}\text { Distribution Coeff. } \\
K\end{array}$ & $\begin{array}{c}\text { Mass Transfer Coeff. } \\
k_{o} a\left(s^{-1}\right)\end{array}$ \\
\hline Sucrose & 0.0079 & 0.0021 \\
Glucose & 0.25 & 0.011 \\
Fructose & 0.59 & 0.016 \\
\hline
\end{tabular}

${ }^{\dagger}$ Eluent - deionized distilled water 


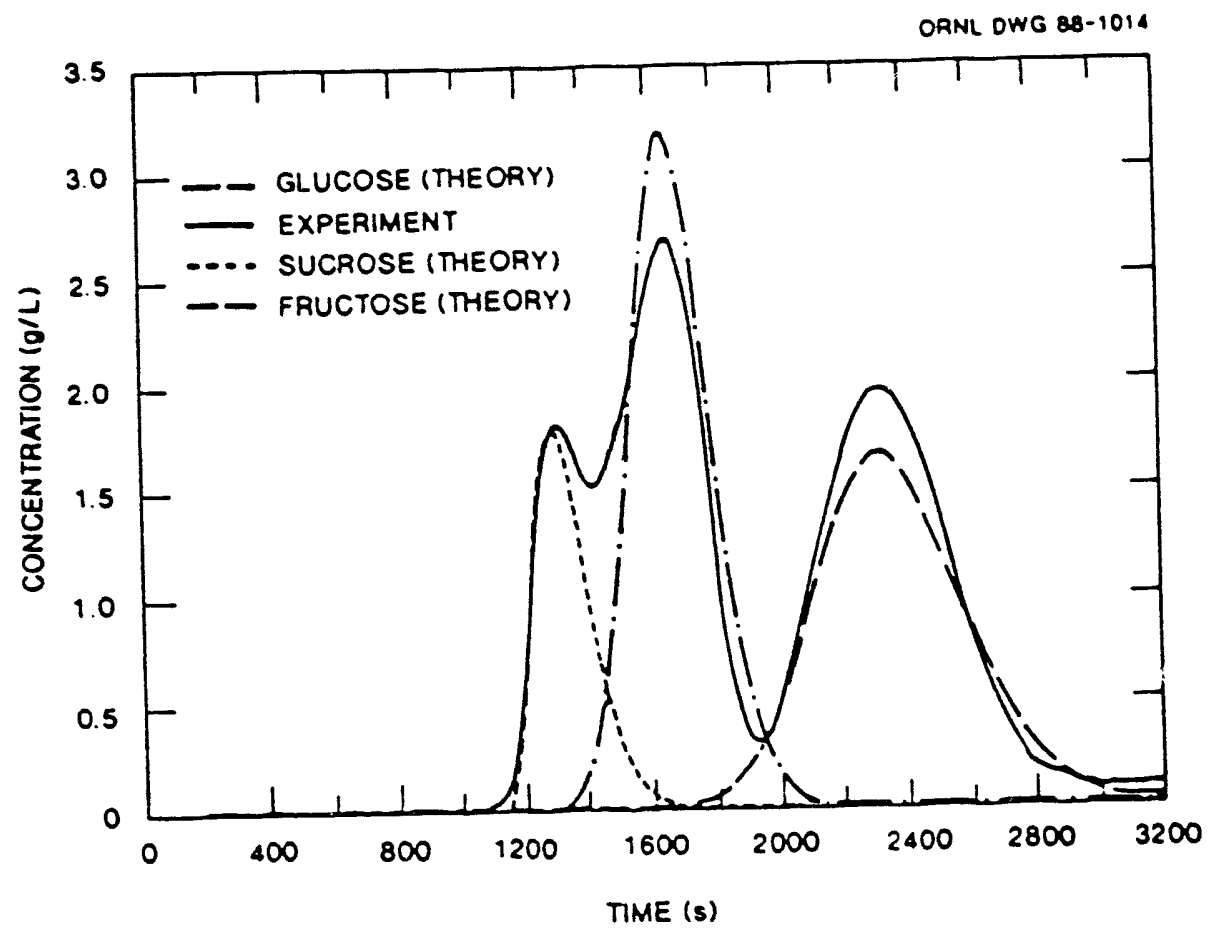

Fig. 2.8 Comparison of experimental fixed-bed results with the prediction of the simple mass transfer resistance theory (Equation 2.4). Feed contains 25 $\mathrm{g} / \mathrm{L}$ glucose, $25 \mathrm{~g} / \mathrm{L}$ fructose, and $10 \mathrm{~g} / \mathrm{L}$ sucrose. 


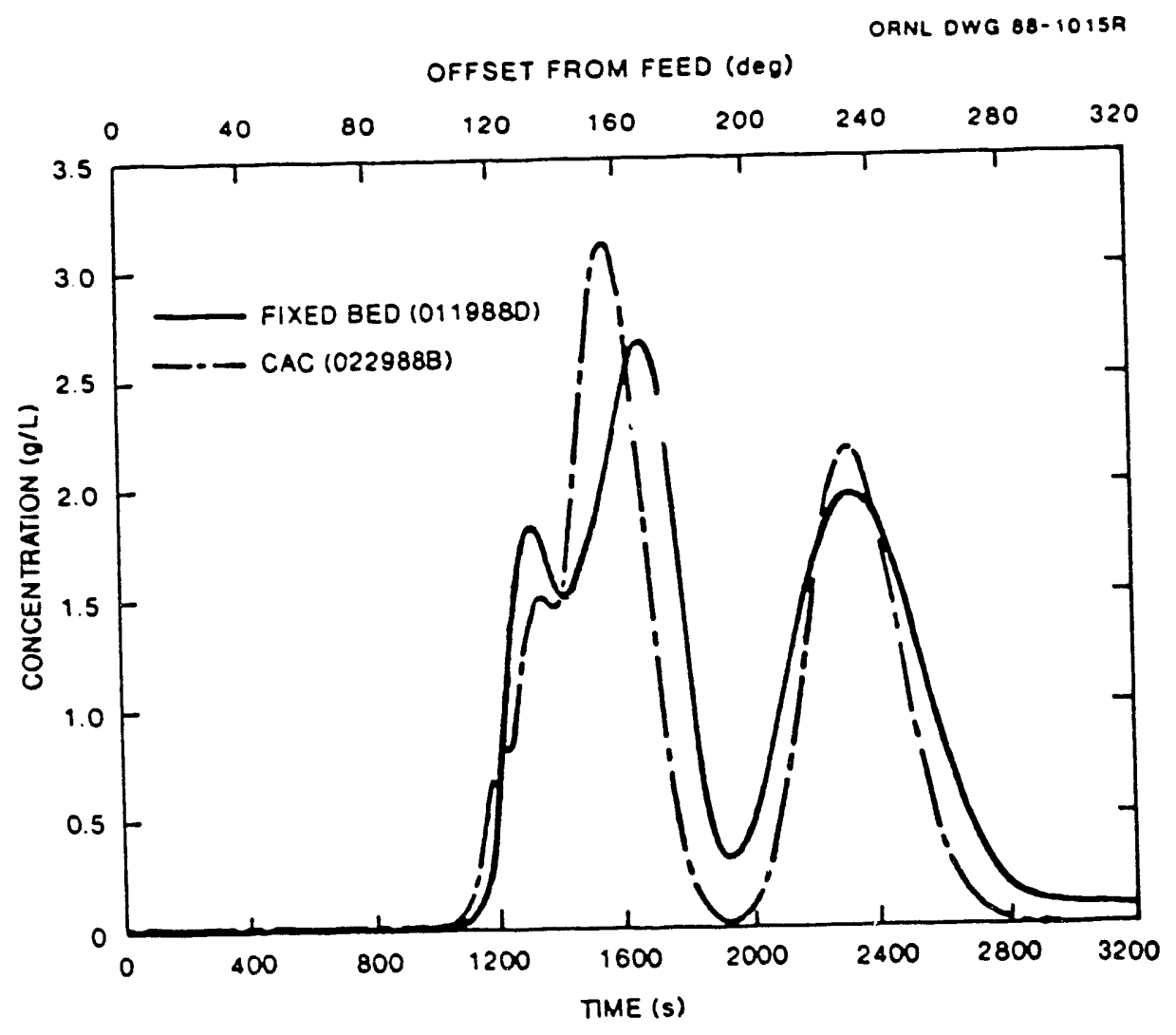

Fig. 2.9 Comparison of experimental fixed-bed results with the equivalent run on the pilot-scale CAC. A synthetic sugar mixture containing $25 \mathrm{~g} / \mathrm{L}$ glucose, $25 \mathrm{~g} / \mathrm{L}$ fructose, and $10 \mathrm{~g} / \mathrm{L}$ sucrose was used in both cases. The CAC feed also contained $1 \mathrm{~g} / \mathrm{L}$ of blue dextran and occupied a $3^{\circ}$ feed sector. The rotation rate was $360^{\circ} / \mathrm{h}$. 
has a molecular weight of 2,000,000 daltons and was excluded from the resin. From its retention angle, the bed voidage fraction, $\epsilon$, was calculated to be 0.39 . A comparison of the fixed-bed and CAC pilot-scale experiments is shown in Fig. 2.9. Both time (lower scale) and angle (upper scale) have been plotted on this graph. Here, we see that the CAC and the fixed-bed run had similar separation performance. In fact, the peak values for glucose and fructose in the CAC experiment were slightly higher than those for the fixed-bed experiment, indicating an apparent better efficiency.

It is far more likely that the fixed-bed experiment contained some dispersion due to less-than-ideal injection of the feed sample. This work confirmed earlier work of Howard et al. (1988) on the same system, but with a smaller size CAC unit.

\subsubsection{Low Concentration - High Loading Experiments}

The industrial feedstock described in Sect. 2.4 was diluted 10:1 with deionized distilled water, resulting in a total sugar concentration of $70.6 \mathrm{~g} / \mathrm{L}$. The viscosity of the eluent and the diluted (10:1) sugar mixture were measured at $25^{\circ} \mathrm{C}$ and found to be $0.97 \mathrm{cp}$ and $1.12 \mathrm{cp}$, respectively. Experiments were performed with the feed sector widths of $30^{\circ}$ and $55^{\circ}$. Implementation of the $30^{\circ}$ feed sector required a single feed nozzle. For the $55^{\circ}$ feed sector, three feed nozzles placed $18.3^{\circ}$ apart were used. To ensure an even flow distribution, each nozzle was fed by an individual metering pump.

Experimental and theoretical concentration profiles using Eq. 2.12 are illustrated for these two runs in Figs. 2.10 and 2.11. There is reasonable agreement between theory and experiment, with the equilibrium and mass transfer parameters calculated from the fixed-bed experiments. The trend in resolution of glucose and fructose in these two wide-feed-sector experiments also agrees with what one would expect: as the loading increases, the resolution between the two peaks decreases. However, with almost twice the loading, a very large per- 


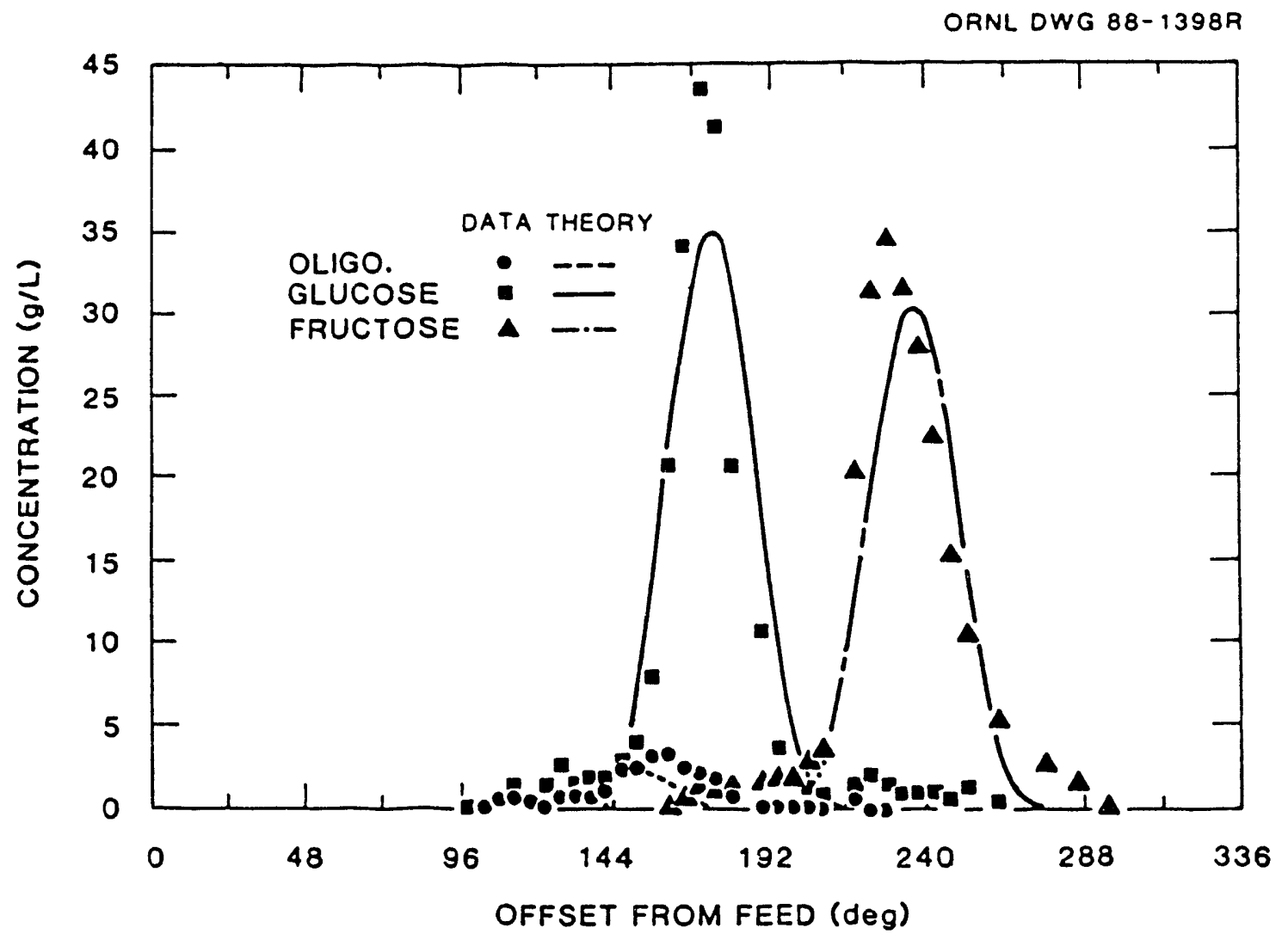

Fig. 2.10 Comparison of experimental $30^{\circ}$ feed sector run with the theoretical predictions of Eq. 2.12. A diluted (10:1)industrial HFCS was used as the feed. The feed viscosity was $1.12 \mathrm{cp}$, while that of the eluent was $0.97 \mathrm{cp}$, both measured at $25^{\circ} \mathrm{C}$. The rotation rate was $144^{\circ} / \mathrm{h}$. 
ORNL DWG 88-1393R

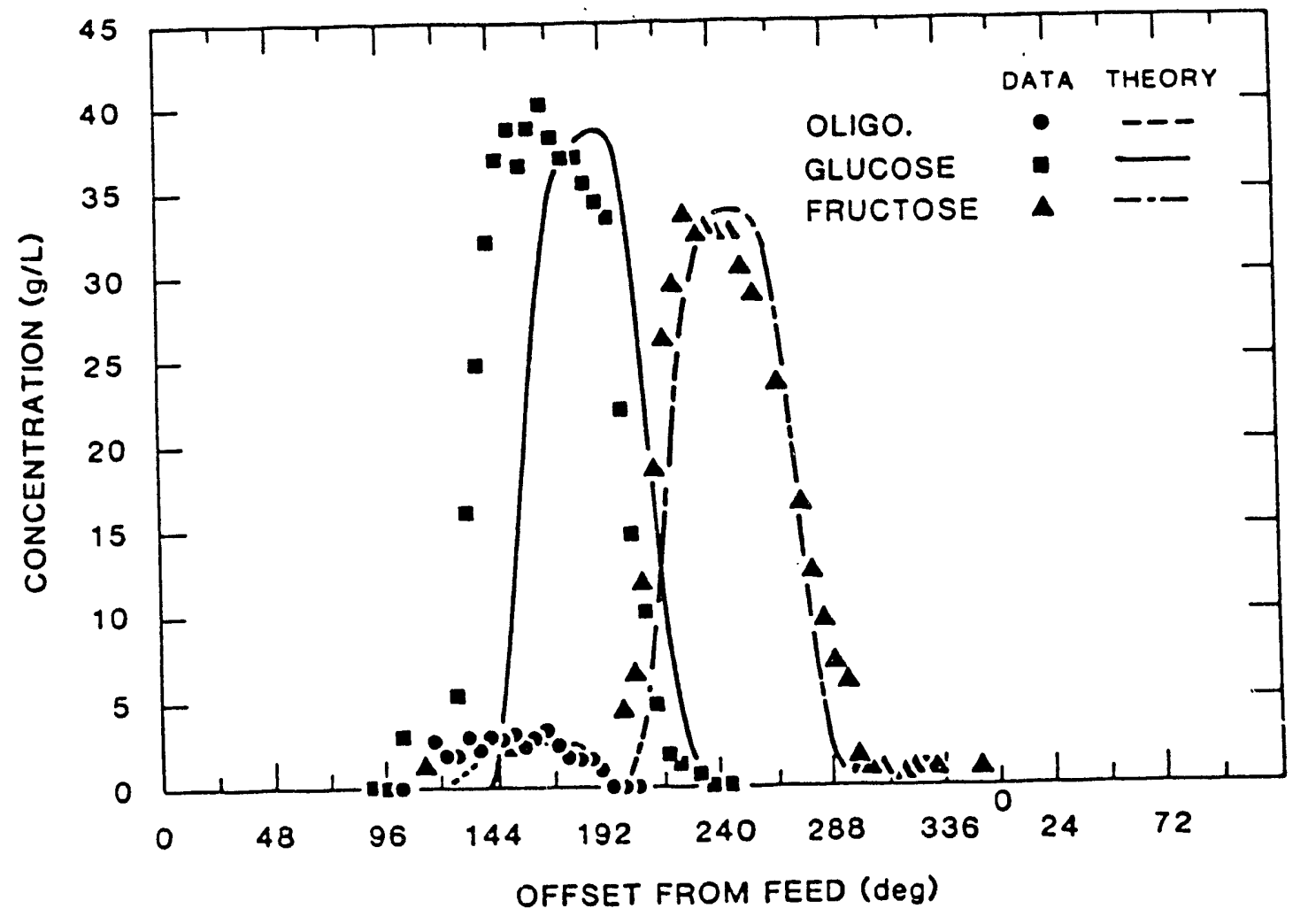

Fig. 2.11 Comparison of experimental $55^{\circ}$ feed-sector run with the theoretical predictions of Eq. 2.12. A diluted (10:1) industrial HFCS was used as the feed. The rotation rate was $144^{\circ} / \mathrm{h}$. 
centage of pure fructose can still be collected. In addition, from comparison of the two figures it is evident that in the higher-feed sector experiment $\left(55^{\circ}\right)$, the experimental peak attains a flat shape at the top showing a plateau region. Note that these plateau regions and their heights are also in approximate agreement with the model. In the lower-feed sector experiment (30॰), the peak is sharp and without a plateau. This is also predicted by theory, although the peak heights are somewhat different. This might be because fixed-bed parameters were used in the model calculations.

\subsubsection{High Concentration - Wide-Feed- Sector Experiments}

A series of runs with increasing feed concentration were made in the pilotscale CAC and are illustrated in Figs. 2.12, 2.13, and 2.14. In each of these runs, the feed sector was $30^{\circ}$ and the feedstocks were a $3: 1$ diluted $(176 \mathrm{~g} / \mathrm{L})$, 1:1 diluted $(353 \mathrm{~g} / \mathrm{L})$ and an undiluted $(706 \mathrm{~g} / \mathrm{L})$ industrial sugar mixture, respectively. The viscosities of these three feeds at $25^{\circ} \mathrm{C}$ were $2.20 \mathrm{cp}, 2.76 \mathrm{cp}$, and $17.7 \mathrm{cp}$. Symbols are experimental values obtained from HPLC analysis, while the solid lines are calculated concentration profiles from Eq. 2.12. The model parameters obtained from the low-concentration experiments were used for these calculations. Thus, these figures effectively show a comparison between the ideal performance obtained in the limit of low concentration (for high or low loading) and the actual performance observed at high feed concentrations. Examining the trend of the three experimental profiles, it is evident that as the feed concentration is increased, the separation performance and resolution are decreased. Figure 2.12 shows excellent agreement between theory and experiment, but ais the concentration is increased, the agreement between theory and experiment breaks down. This is readily evident in Fig. 2.14, which predicts product peak

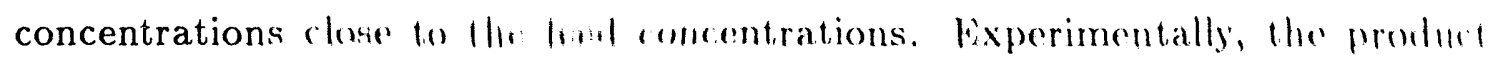

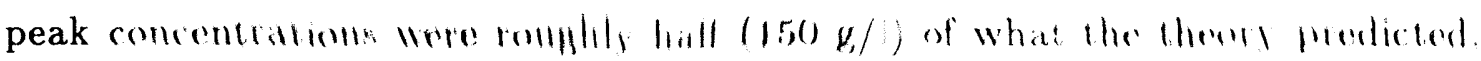

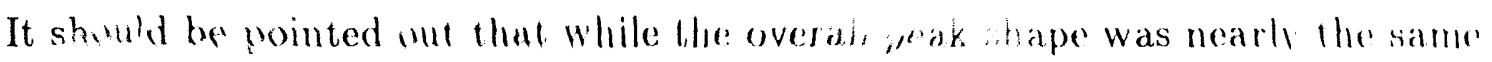


ORNL DWG 89-78

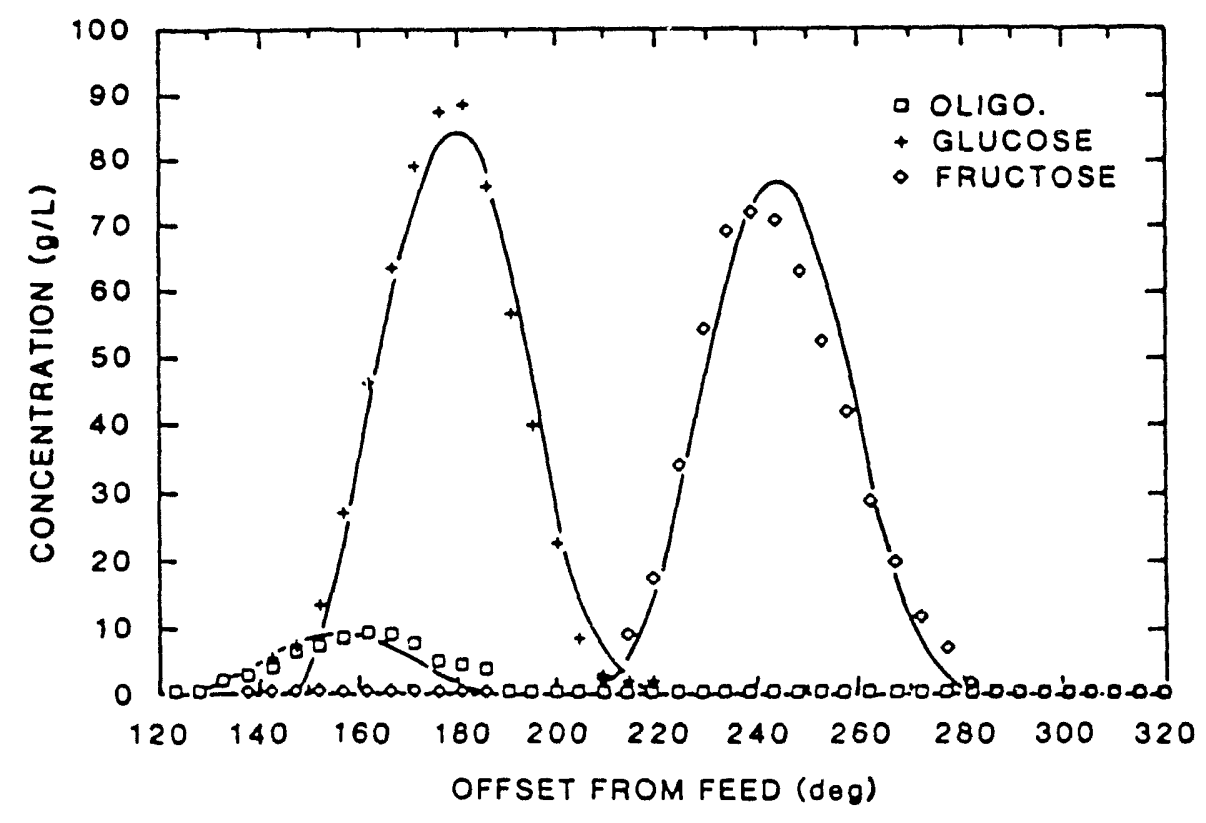

Fig. 2.12 Comparison of experimental $30^{\circ}$ feed-sector run with the theoretical predictions of Eq. 2.12. A diluted $(3: 1)$ industrial HFCS was used as the feed. The feed viscosity was $2.2 \mathrm{cp}$, while that of the eluent was $0.97 \mathrm{cp}$, both measured at $25^{\circ} \mathrm{C}$. The rotation rate was $144^{\circ} / \mathrm{h}$. 


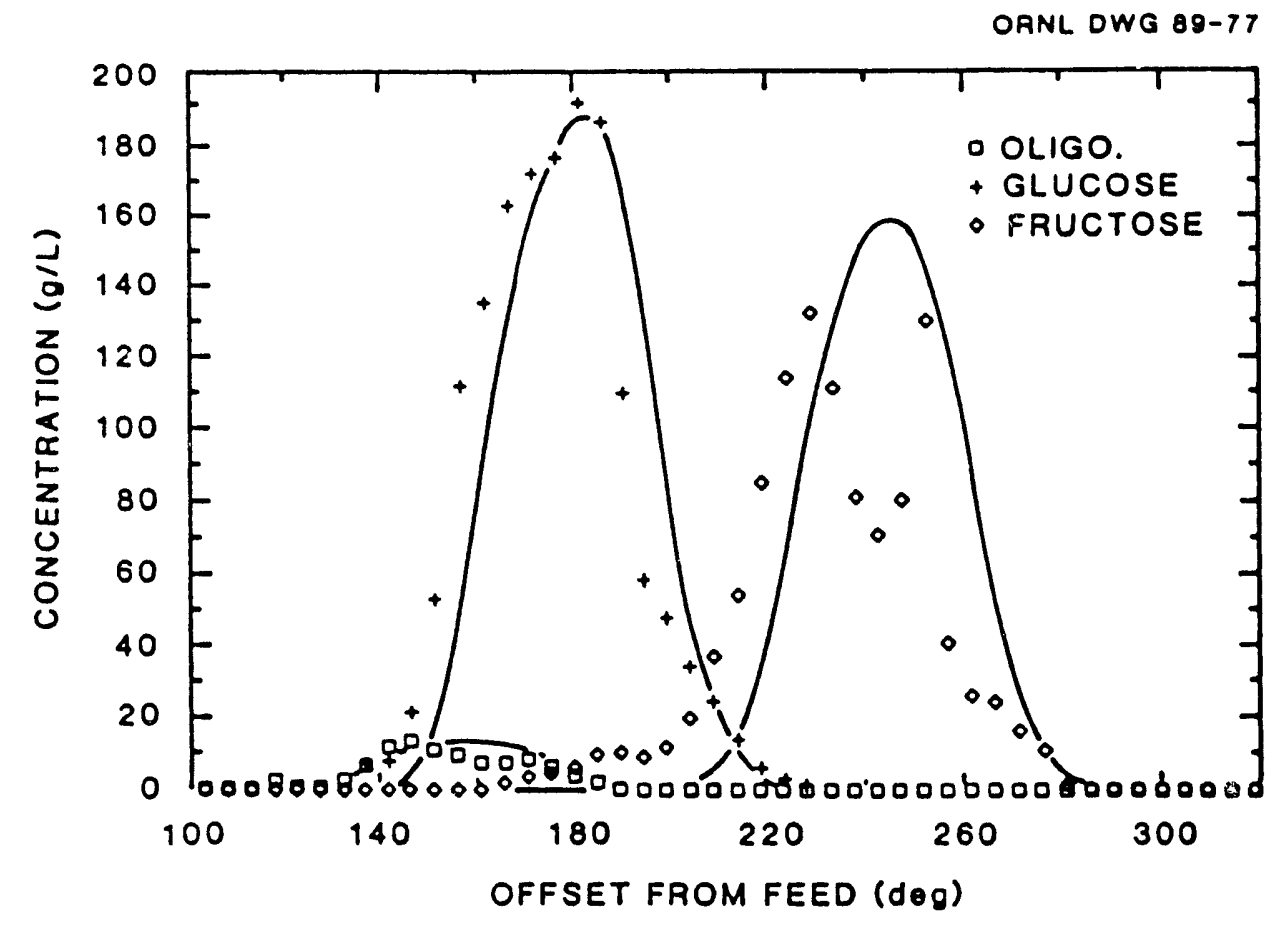

Fig. 2.13 Comparison of experimental $30^{\circ}$ feed-sector run with the theoretical predictions of Eq. 2.12. A diluted (1:1) industrial HFCS was used as the feed. The feed viscosity was $2.76 \mathrm{cp}$, while that of the elvent was $0.97 \mathrm{cp}$, both measured at $25^{\circ} \mathrm{C}$. The rotation rate was $144^{\circ} / \mathrm{h}$. 
ORNL DWG 89-76

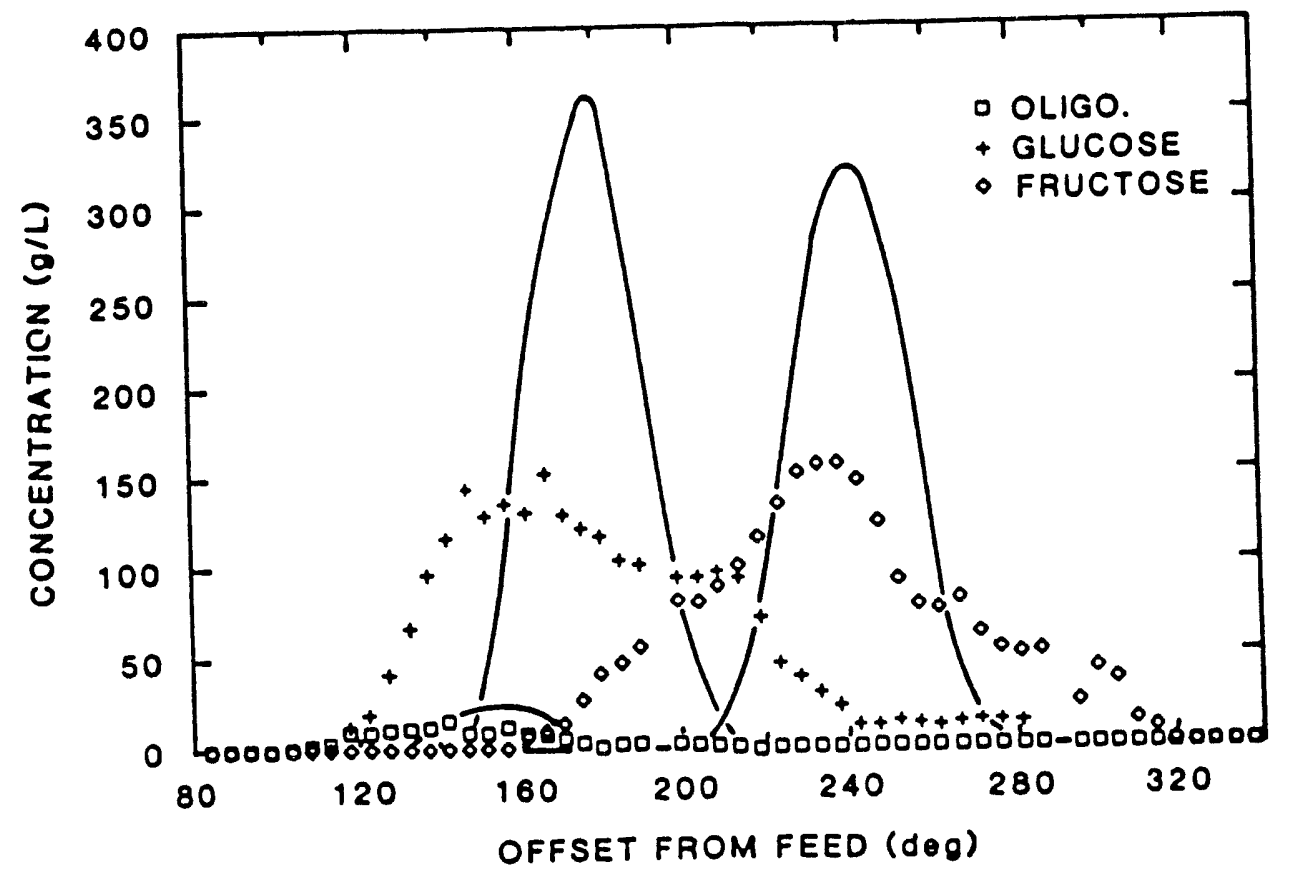

Fig. 2.14 Comparison of experimental $30^{\circ}$ feed-sector run with the theoretical predictions of Eq. 2.12. An undiluted industrial HFCS was used as the feed. The feed viscosity was $17.7 \mathrm{cp}$, while that of the eluent was $0.97 \mathrm{cp}$, both measured at $25^{\circ} \mathrm{C}$. The rotation rate was $144^{\circ} / \mathrm{h}$. 
in these and duplicate experiments, there were some differences from run to run in the waving "zig-zag" profiles obtained at higher feed viscosities. These differences, as well as the differences between the experimental and theoretical profiles, are most likely attributed to the increase in the feed viscosity (the feed viscosity for the run in Fig. 2.14 is over 17 times that of the eluent). This effect is not limited to $\mathrm{CAC}$ operation. Similar results have been found, for example, for high loadings in fixed-bed gel permeation chromatography columns. Viscous fingering, as it is called, results from hydrodynamic instability of the viscous zones (Heftman, 1975). In viscous fingering, the drop in viscosity in the trailing edge of a peak allows the eluent to "punch through" ahead of the feed band while "fingers" of the sample are delayed (Moore, 1970). Altgelt (1970) found that reasonably good separations in overloaded columns could be performed if the feed-to-eluent viscosity ratio is not above 2 . Heftman (1975) found poor sample resolution if this ratio becomes greater than 3 to 5 . The experiments performed on the $\mathrm{CAC}$ tend to agree with these results. If the feed-to-eluent viscosity ratio rises above 2, the separation performance deteriorates significantly.

Industrial operations of sugar separations attempt to mitigate the problems of large viscosity differences by increasing the operatıng temperature. For example, at $80^{\circ} \mathrm{C}$, the viscosity of the undiluted HFCS falls to $3.5 \mathrm{cp}$, but a feed-to-eluent viscosity ratio of 9 still exists.

While this will help alleviate the problem, it may not completely solve it. In addition, by operating at higher temperatures, the distribution coefficient of fructose is reduced, while that of glucuse remains unchanged (Ganetsos, 1987). This is most likely due to a shift in solution equilibrium toward the production of a fructose with a higher furanose and lower pyranose composition. In any case, the advantages of reduced viscosity tend to outweigh those of a decreased separation coefficient.

In order to determine if viscous spreading was enhanced by the CAC, a fixedbed experiment was made with the undiluted HFCS at conditions that corresponded to the $\mathrm{CAC}$ experiment. The concentration profiles for these two 


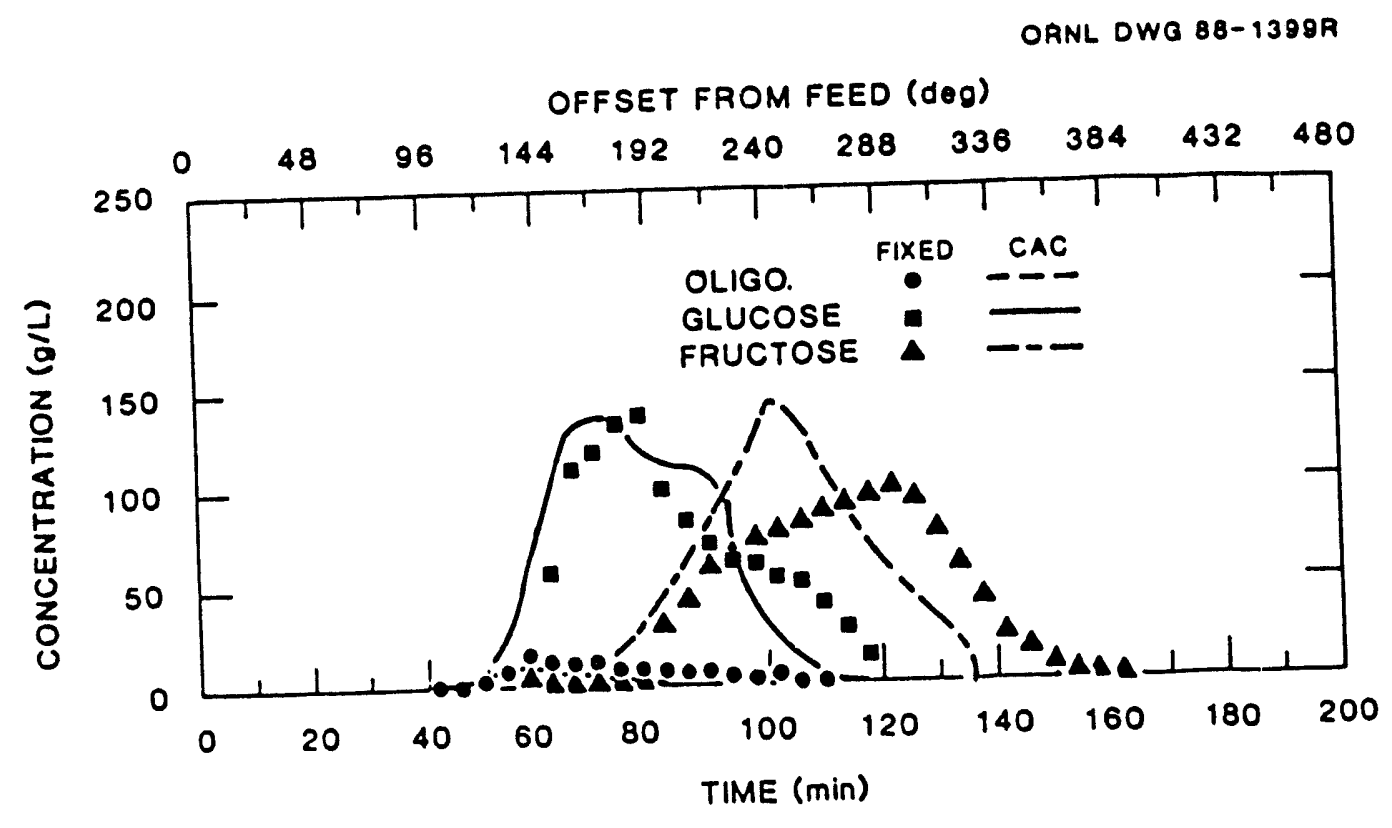

Fig. 2.15 Comparison of experimental CAC $30^{\circ}$ feed-sector run with an equivalent fixed-bed column run. An undiluted industrial HFCS was used as the feed. The feed viscosity was $17.7 \mathrm{cp}$, while that of the eluent was $0.97 \mathrm{cp}$, both measured at $25^{\circ} \mathrm{C}$. 
experiments are shown in Fig. 2.15. While the agreement between the two runs in Fig. 2.15 is not as good as the two runs in Fig. 2.9, it does indicate that the effects of large differences in feed and eluent viscosity do not become more pronounced in the CAC.

Finally, an experiment which used a $72^{\circ}$ feed and the undiluted feed stock with a viscosity of $17.7 \mathrm{cp}$ was performed. Concentration profiles of the experimental run and the theoretical prediction are given in Fig. 2.16. It is readily apparent that viscosity effects which were previously discussed are present in this experiment. Note, however, that the peak concentrations of glucose and fructose in the prediction of the concentration profile indicate that at high loading, such as in this run, the dilution due to isocratic elution can be reduced at the expense of reduced purity.

\subsection{SUMMARY AND CONCLUSIONS}

The separation of glucose, fructose, and sucrose on the calcium form of Dowex $50 \mathrm{~W}-\mathrm{X} 8$ has been demonstrated in both fixed-bed columns and a pilot- scale CAC. Equilibrium distribution coefficients and mass transfer parameters were determined on the fixed bed and used in the models for prediction of CAC concentration profiles. Fixed-bed experiments with a small feed pulse and pilotscale CAC experiments with a small feed sector were virtually identical and could be predicted fairly accurately. This indicates that for low-concentration, lightly loaded systems, the CAC can be scaled up efficiently from fixed-bed experiments.

For the highly loaded low-concentration experiments, there was general agreement between the theory and the experiment and no "nonideal" effects were observed. Thus, scaleup from fixed bed to CAC can be accomplished for highly loaded systems. However, when the feed concentration was increased to increase the column loading, there was significant deviation from the "ideal" beinavior of the low concentration experiments. Most likely these were due to viscous effects 


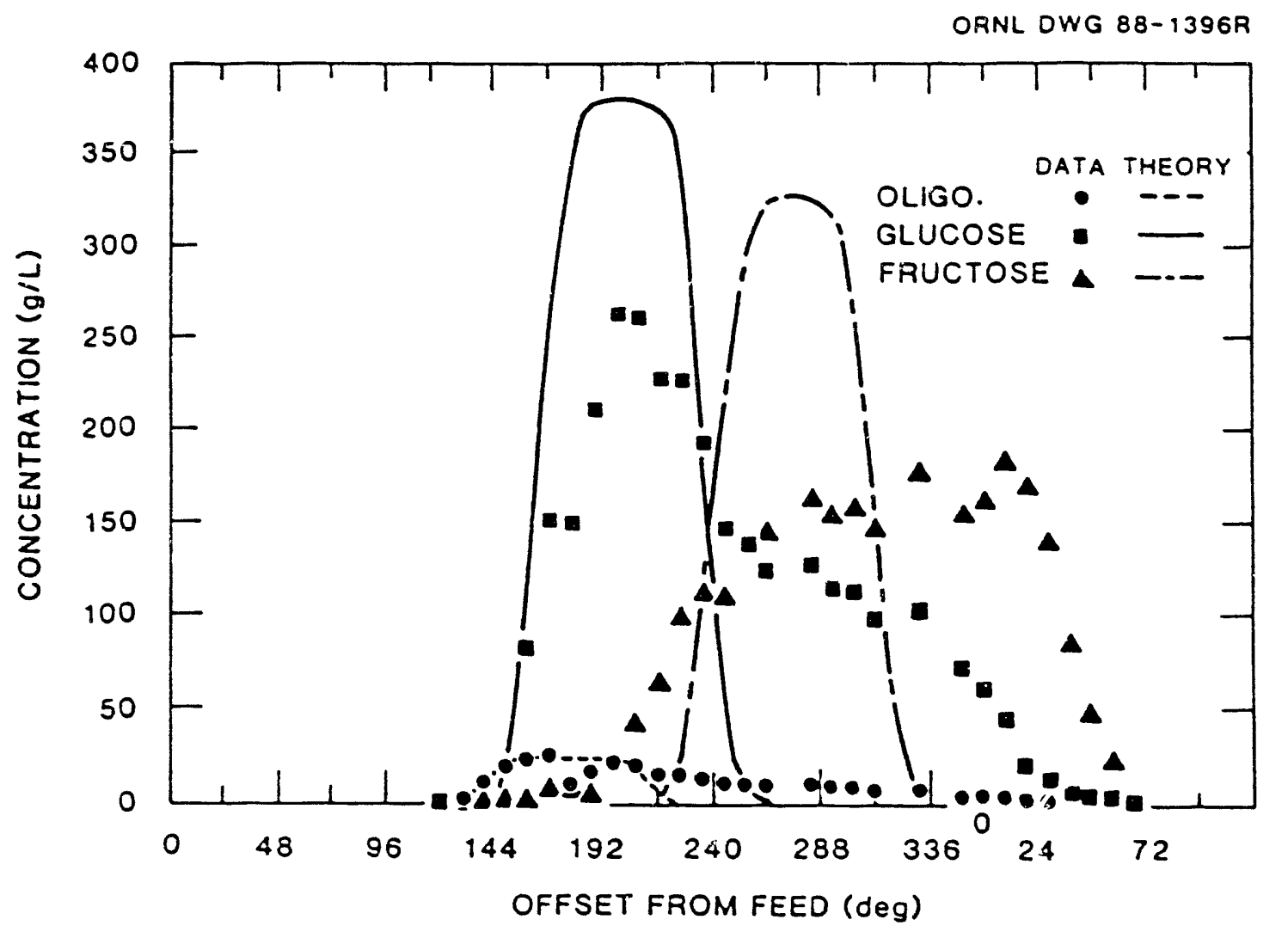

Fig. 2.16 Comparison of experimental $72^{\circ}$ feed-sector run with the theoretical predictions of Eq. 2.12. An undiluted industrial HFC 5 was used as the feed. The feed viscosity was $1.12 \mathrm{cp}$, while that of the elue'،t was $0.97 \mathrm{cp}$, both measured at 2000 . 
caused by the increased sugar concentrations. When the feed-to-eluent viscosity ratio climbs above 2 , the separation performance deteriorates because of flow nonuniformities. Flow nonuniformity was not restricted to the pilot-scale CAC and was exhibited in the fixed column as well.

In industry, sugar separation columns are operated at 60 to $85^{\circ} \mathrm{C}$. The increase in temperature causes a decrease in the viscosity ratio of sugar and eluent but does not completely alleviate the problem.

In conclusion, it has been proven that, the $\mathrm{CAC}$ can be scaledup from fixedbed experiments provided that in scaling, the system nonideal effects such as dispersion and flow nonuniformities are not introduced. The largest throughput obtained with the pilot-scale unit was approximately $180 \mathrm{~L}$ of feed per day with the low-concentration, $55^{\circ}$ feed experiment (Fig. 2.11). This separation could be very accurately predicted on the basis of an experiment carried out in a 0.95-cm-ID fixed-bed column. 


\section{SEPARATION OF METALS BY STEP ELUTION}

\subsection{INTRODUCTION}

In the past, the majority of the research on the CAC has been dedicated to isocratic separations (i.e., separations using a single eluent). This method is capable of providing high-resolution multicomponent separations but inevitabiy causes some dilution of the separated products. In order for CAC to become important for certain industrial applications, it is necessary to demonstrate that it can be operated in a mode that will not cause significant dilution. One such mode of operation is gradient, or stepwise, elution. Through judicious choice of eluent systems it should be possible, as in fixed-bed operations, to decrease dilution by changing the eluent "strength." The advantages of operating with various eluents is particularly evident in the separation of multicomponent mixtures when the different components have vastly different separation factors. Consider, for example, a three-component mixture, consisting of $\mathrm{A}, \mathrm{B}$, and $\mathrm{C}$, and assume that $A$ and $B$ are weakly sorbed by the chromatographic medium while $\mathrm{C}$ is strongly sorbed. In this case, a long chromatographic column will be needed to separate A and B. However, because $C$ is strongly sorbed, it will exit from such a long column after a lengthy period of time and will be considerably diluted. This will reduce column throughput and product recovery. A step (or increase) in eluent strength can be used here to speed up the elution of the strongly retained species after $A$ and $B$ are separated. In this manner the column throughput will be increased and product dilution minimized.

A few preliminary experiments on gradient elution have been performed at ORNL. Canon et al. (1980) performed a preliminary stepwise elution experiment on a system containing a copper-nickel-cobalt mixture, and Begovich and Sisson (1983) performed some work on stepwise elution in the separation of zirconiumhafnium mixtures. From these preliminary studies it was observed that (1) the dilution was considerably reduced, and (2) the efficiency of the column utilization was improved. The purpose of this study, therefore, was to determine column 
conditions that would give these advantages and to quantify the improvements that might be attained at these conditions. The experimental separation system consisted of low-concentration iron and chromium mixtures that are similar to the effluents from dump and heap leaching of dilute ores used in hydrometallurgy. [At Kennecotts Bingham copper mine, for example, effluent solutions contain 0.5 g/L copper and are typically separated by ion exchange (Wadsworth, 1987).] In addition, the iron-chromium system provided ease of analysis and yielded colored bands that could be visually observed through the acrylic wall of the CAC apparatus.

\subsection{EXPERIMENTAL METHODS}

The CAC used in these experiments was similar to the one used by Howard et al. (1988) to study the separation of sugars. The unit was modified to allow step elution. Details of the apparatus are shown in Fig. 3.1. The apparatus was constructed of clear Plexiglas to allow visual observation of ongoing experiments. It was 60-cm high, had an OD of $30.5 \mathrm{~cm}$, and an annulus width of $1.27 \mathrm{~cm}$. The bed was packed to a depth of $50 \mathrm{~cm}$ with Dowex $50 \mathrm{~W}-\mathrm{X} 8$ ion-exchange resin. A layer of $0.018-\mathrm{cm}$-diam glass beads was placed on top of the resin bed to a depth of $10 \mathrm{~cm}$. Eluents and feed were introduced at the top of the column through a stationary inlet distributor that extended inio the head space of the column through two Teflon O-rings. This allowed the apparatus to rotate while the feed and eluent distributors remained fixed in space. One eluent, typically the one with the highest flow rate, was allowed to flocd the head space while the remaining eluent (the step eluent) and the fec $\dot{\mathrm{u}}$ mixture were introduced through stainless steel nozzles whose tips were located with in the glass bead layer. The glass bead layer was sufficiently fluid to prevent the nozzles from carving a permanent trench, thus creating a self-healing upper layer. Tracer experiments, using blue dextran as a visible dye, demonstrated that an even 


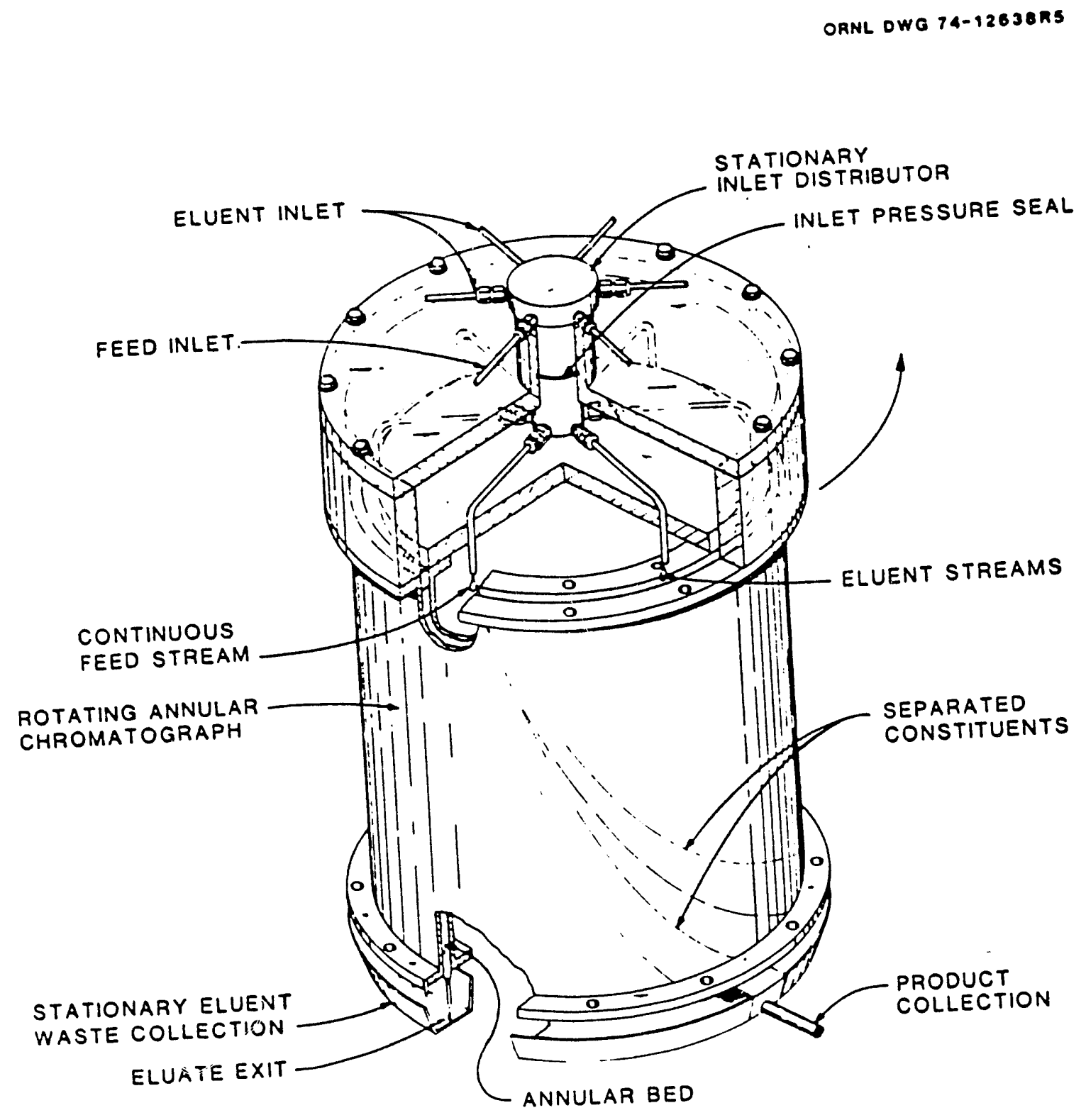

Fig. 3.1 Laboratory-scale CAC unit. 
distribution of Auid within the glass bead layer could be attained which would prevent convective mixing of adjacent fluids. Each eluent and feed quickly spread from the nozzles' tips, acquiring the same superficial velocity, and, at a short distance from the nozzles, became evenly distributed in adjacent sectors of the annulus occupying areas proportional to their flow rate. The maximum flow through each nozzle was determined with this tracer system so that eluent flow in a particular annular segment would not overflow into the head space. The feed was delivered by an Alltech Model 110A positive displacement pump, and the eluent by a Milroyal Model DC-1-175R pump. All pumps were rated for $1 \%$ or better flow accuracy.

A digitally controlled drive system was used to slowly rotate the entire column assembly. The column contained 180 exit ports, fit with high-density polyethylene plugs to provide support for the sorbent bed. Each port was fitted with a short stainless steel tube to prevent drop coalescence at the column's exit. Product could be collected at the exit by placing stationary collection pans below the column at angles corresponding to those of the product effluent.

Concentration profiles at the column exit could be obtained during steady operation by connecting an in-line, continuous detection instrument to a single eluate exit tube. This method has been described in Sect. 2.4. In practice, a UV/Vis Spectrophotometer (Perkin-Elmer, Model 200) was used as the detector that received eluate from a peristaltic pump set to a flow rate $1 / 180$ of the total flow through the CAC. The millivolt signal from the detector was converted to a digital signal by an A/D conversion board (Data Translation, Model 2805) interfaced to a microcomputer (IBM-PC AT) for graphical display and data storage. Calibration curves for $\mathrm{Fe}^{3+}$ and for $\mathrm{Cr}^{3+}$ were obtained at $520 \mathrm{~nm}$ and $620 \mathrm{~nm}$, respectively. The tracer, blue dextran 2000 (Pharmacia), was detected at $650 \mathrm{~nm}$.

The feed components, obtained from Fisher, were ferric and chromic nitrate salts. Mixtures typically contained $5.0 \mathrm{~g} / \mathrm{L} \mathrm{Fe}^{+3}$ aind $5.0 \mathrm{~g} / \mathrm{L} \mathrm{Cr}^{+3}$ and were prepared by dissolving the nitrate salts in $0.4 \underline{\mathrm{M}}$ ammonium sulfate solutions. 
The main eluent, which was allowed to flood the head space, contained $0.4 \underline{\mathrm{M}}$ ammonium sulfate and $0.025 \mathrm{M}$ sulfuric acid. Step eluents varied in concentration from $0.4 \underline{\mathrm{M}}$ to $1.5 \underline{\mathrm{M}}$ ammonium sulfate (at a constant $0.025 \underline{\mathrm{M}} \mathrm{H}_{2} \mathrm{SO}_{4}$ ). Dowex $50 \mathrm{~W}-\mathrm{X} 8$ resin in the ammonium form with an average particle size of 50 $\mu \mathrm{m}$ was used for all experiments.

In most runs, the step eluent nozzle was placed $35^{\circ}$ from the feed nozzle within the glass bead layer and step eluent was pumped in the range from $12^{\circ}$ to $58^{\circ}$. The implementation of such a system is illustrated in Fig. 3.2, which shows an "unwrapped" section of the CAC unit. As shown in this pictorial view that is based on experimental observations, the two components initially travel rapidly and form well-separated bands. As these bands come to interfere with the more concentrated eluent, however, they are deflected and elute from the column at a much shorter distance from the feed point than they would if a single dilute eluent were used. While the bands are still separated, the product dilution is significantly less than what would have been obtained with a simple low-concentration eluent. The column throughput is also increased since a larger feed flow rate or multiple feeds can now be used. Isocratic runs could also be made in the system if the step eluent was not used and the main eluent was allowed to cover the entire circumference of the bed.

A number of process parameters were varied independently, including the step eluent concentration, the rotation rate, the feed concentration and feed flow rate, and the step eluent position relative to the feed point. A number of experiments were also carried out in a fixed-bed column, similar to the one described in Sect. 2.4, to determine equilibrium and mass transfer parameters for the two metal ions. The fixed-bed apparatus in this case consisted of a glass column $1.5 \mathrm{~cm}$ in diameter and 40-cm long (Glenco). The column was fitted with polypropylene end caps with Teflon mesh screen at the bed bottom and a plunger-type distributor at the top. The eluent was delivered to the column by a positive displacement pump (FMI) and feed samples were introduced with a six-port Reodyne sample valve. The effluent was monitored (as in the CAC 


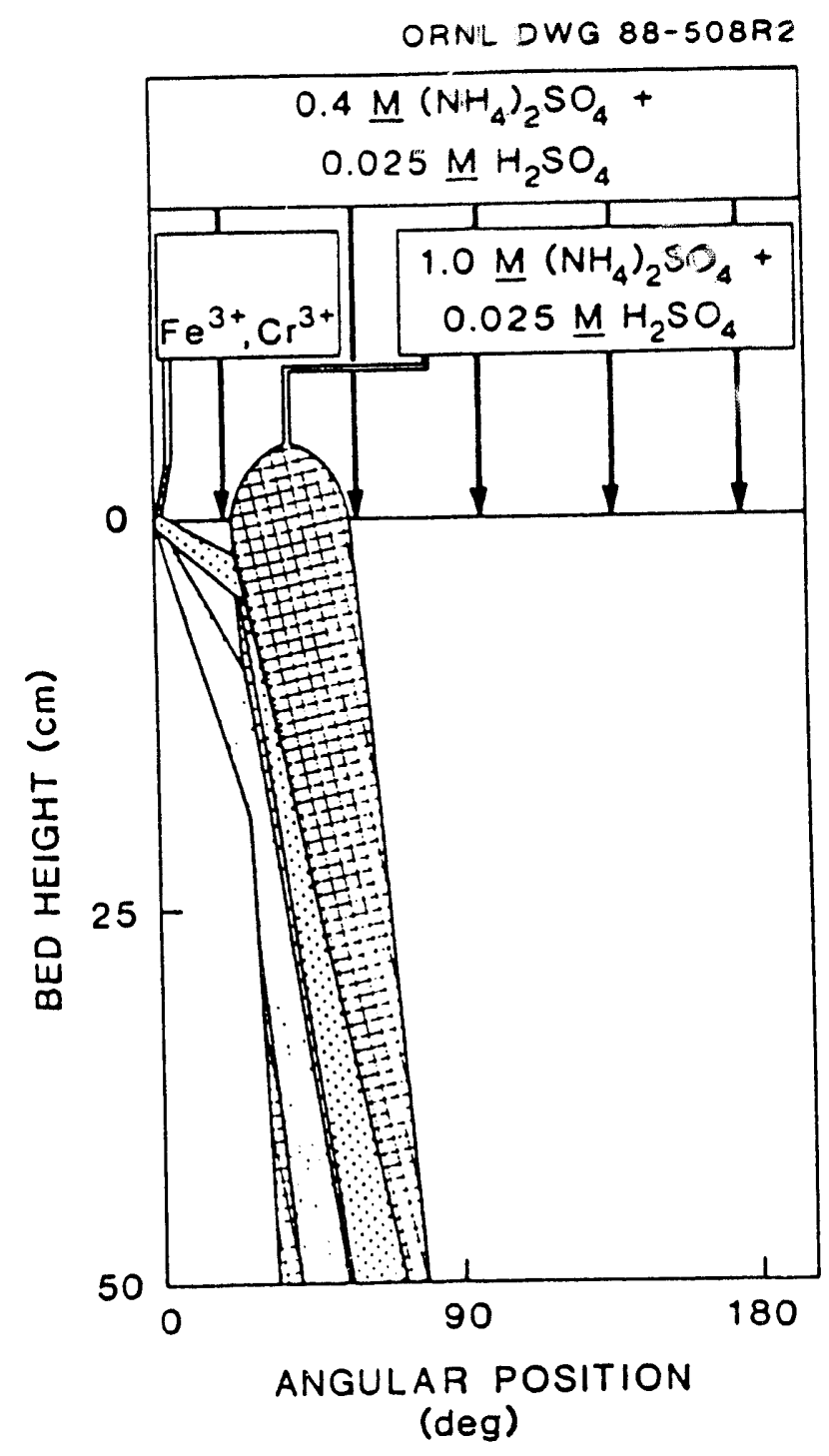

Fig. 3.2 Arrangement of feed and eluents in step-elution operation. 
experiments) with a Varian spectrophotometer connected to the microcomputer for data acquisition.

\subsection{THEORETICAL DISCUSSION}

Isocratic behavior in the CAC has been extensively modeled by Begovich (1982) and Howard et al. (1988) and may be described as discussed in Sect. 2.5. For the CAC, a steady-state, solute material balance in the cylindrical coordinate system may be described by the following equation:

$$
\epsilon D_{e} \frac{\partial^{2} C}{\partial z^{2}}=\omega \epsilon \frac{\partial C}{\partial \theta}+\omega(1-\epsilon) \frac{\partial q}{\partial \theta}+\epsilon v \frac{\partial C}{\partial z},
$$

where $D_{e}$ is an effective dispersion coefficient characteristic of the CAC. Using a solid-film transport model, the fluid-and solid-phase concentrations can be related by the following equation:

$$
\omega(1-\epsilon) \frac{\partial q}{\partial \theta}=k^{\prime}\left(q^{*}-q\right)
$$

where $\mathrm{k}^{\prime}$ is a solid-film diffusion coefficient and $q^{*}$ is the sorbent concentration that would be in equilibrium with the fluid concentration $C$. For a system of dilute metal ions, linear equilibrium can be assumed in the presence of an excess of ammonium sulfate. In this case,

$$
q^{*}=K C
$$

where $K$ is the equilibrium distribution coefficient that depends on the eluent concentration $C_{0}$. The approximate solution describing the response to an infinitesimal feed pulse presented in Sect. 2.5 may be written in terms of $k^{\prime}$ as follows (Sherwood et al., 1975):

$$
C(z, \hat{t})=\frac{Q}{2 \sqrt{\pi}}\left\{\frac{\left(k^{\prime}\right)^{2}}{u^{3} z K \hat{t}(1-\epsilon)^{3}}\right\}^{0.25} \exp \left\{-\left[\sqrt{\frac{k^{\prime} K z}{u}}-\sqrt{\frac{k^{\prime} \hat{t}}{1-\epsilon}}\right]^{2}\right\},
$$


where $Q$ and $\hat{t}$ are defined for the CAC as

$$
Q=\frac{C_{F} u Q_{F}}{Q_{T}} \frac{360^{\circ}}{\omega}
$$

and

$$
\hat{t}=\frac{\theta}{\omega}-\frac{\epsilon z}{u}
$$

In these equations, $C_{F}$ is the solute feed concentration, $Q_{F}$ is the feed flow rate, and $Q_{T}$ is the total flow rate of fluid through the annular bed. The equilibrium distribution coefficient, $K$, and the mass transfer parameter, $k^{\prime}$, may be calculated from pulse experiments using

$$
\begin{gathered}
\frac{k^{\prime} K z}{u}=16(\ln 2)\left(\frac{\hat{t}_{\mathrm{max}}}{\Delta}\right)^{2}, \\
K=\frac{u \hat{t}_{\mathrm{max}}}{(1-\epsilon) z} .
\end{gathered}
$$

The "time" at which the maximum concentration occurs, $\hat{t}_{\mathrm{m} a x}$, is given by

$$
\hat{t}_{\mathrm{max}}=\frac{\theta_{\mathrm{m} a x}}{\omega}-\frac{\epsilon z}{u},
$$

and $\Delta$ is the width of the chromatographic peak at half the maximum peak height.

The model was modified to predict the performance of chromatographic operations involving step elution (Carta, 1988). In this case, a material balance must be written for the step eluent as well as for the solute since the resin is almost entirely in the ammonium form and the sulfate coion is excluded by the Donnan potential effect. A material balance on ammonium sulfate may be written as follows:

$$
\epsilon D_{e} \frac{\partial^{2} C_{s}}{\partial z^{2}}=\omega \epsilon \frac{\partial C_{s}}{\partial \theta}+\epsilon v \frac{\partial C_{s}}{\partial z}
$$

Since solid-phase accumulation is neglected in this equation, the ammonium sulfate step concentration is predicted to travel through the column at a velocity essentially equal to the fluid velocity. Equations 3.1, 3.2, and 3.10 are related 
through the dependence of the equilibrium distribution coefficient on step eluent concentration and must be solved simultaneously. If $C_{s}^{0}$ and $C_{8}^{1}$ are the initial and step eluent concentrations, the initial and boundary conditions are

$$
\begin{aligned}
& z=0: \quad 0<\theta<\theta_{F}, \quad C-\frac{D_{e}}{v} \frac{\partial C}{\partial z}=C_{F} \\
& \theta>\theta_{F}, \quad C-\frac{D_{e}}{v} \frac{\partial C}{\partial z}=0 \\
& 0<\theta<\theta_{s}, \quad C_{s}-\frac{D_{e}}{v} \frac{\partial C_{s}}{\partial z}=C_{s}^{0}, \\
& \theta>\theta_{\mathrm{s}}, \quad C_{\mathrm{s}}-\frac{D_{e}}{v} \frac{\partial C_{\mathrm{s}}}{\partial z}=C_{\mathrm{s}}^{1} \\
& z=Z: \quad 0>\theta>0, \quad \frac{\partial C}{\partial z}=0, \quad \frac{\partial C s}{\partial z}=0 \\
& \theta=0: \quad 0<z<Z, \quad C=0, \quad C_{s}=C_{s}^{0}
\end{aligned}
$$

where $\theta_{F}$ is the length of the feed sector (in degrees) and $\theta_{\theta}$ is the angle at which the step in eluent is applied, measured from the feed point. The same effective dispersion coefficient is used for the solute and the step eluent species.

A solution of Eqs. 3.1, 3.2, and 3.10 with these boundary conditions was obtained numerically by using the method of orthogonal collocation (Carta, 1988; Villadsen and Michelssis, 1978). The concentration variables $q, C$, and $C_{s}$ were discretized in the axial direction using Lagrange interpolation polynomials, choosing as the collocation points the zeroes of Jacobi polynomials $P_{N}^{(\alpha, \beta)}(z / Z)$, with $\alpha$ and $\beta$ equal to zero. Twenty interior collocation points were used to carry out the numerical solution. The resulting system of ordinary differential equations was integrated using Gear's method.

The concentration profiles were calculated using values of the equilibrium distribution and mass transfer coefficients determined from Eqs. 3.7 and 3.8. The axial dispersion coefficient was estimated from the measurements of Howard et al. (1988) and was discussed in Sect. 1.4. According to these results the Peclet rumber remains approximately constant for the range of flow rates used in the 
experiments and is equal to

$$
\varepsilon P e=\frac{d_{\mathrm{p}} u}{D_{e}}=0.1,
$$

where $d_{p}$ is the diameter of the resin particles. Calculations using values of $D_{e}$ obtained from this equation showed that very minimal dispersion of the feed species occurs in the apparatus. Instead, some spreading of the step eluent band is predicted.

\subsection{RESULTS AND DISCUSSION}

\subsubsection{Fixed-Bed Experiments}

Isocratic experiments were carried out in the fixed-bed column to determine equilibrium and mass transfer parameters from Eqs. 3.7 and 3.8. The equilibrium distribution coefficient, $K$, determined from Eq. 3.8, was found to be independent of the concentration of iron and chromium (i.e., linear equilibrium) at constant eluent concentration for metal ions in the range 0 to $10 \mathrm{~g} / \mathrm{L}$. As illustrated in Fig. 3.3, however, increasing the eluent concentration, $\mathrm{C}_{s}$, decreases the distribution coefficient, $K$. Such strong dependence of $K$ is due to the competition of the ammonium ion for the functional groups on the resin that are responsible for the uptake of the two metal ions. The $\mathrm{K}$ values were correlated empirically with the following expression:

$$
K=\alpha\left(C_{s}\right)^{\beta}
$$

where $\alpha$ and $\beta$ are empirical constants. Values of $\alpha$ and $\beta$ were determined from a least squares fit of the data and are given in Table 3.1. In addition, the mass transfer coefficient, $\mathrm{k}^{\prime}$, was found to be approximately independent of eluent concentration and flow rate. Lack of flow rate dependence indicates that solid-phase resistance is indeed the rate-controlling mechanism. The values of the mass transfer parameters for iron and chromium are also given in Table 3.1. 


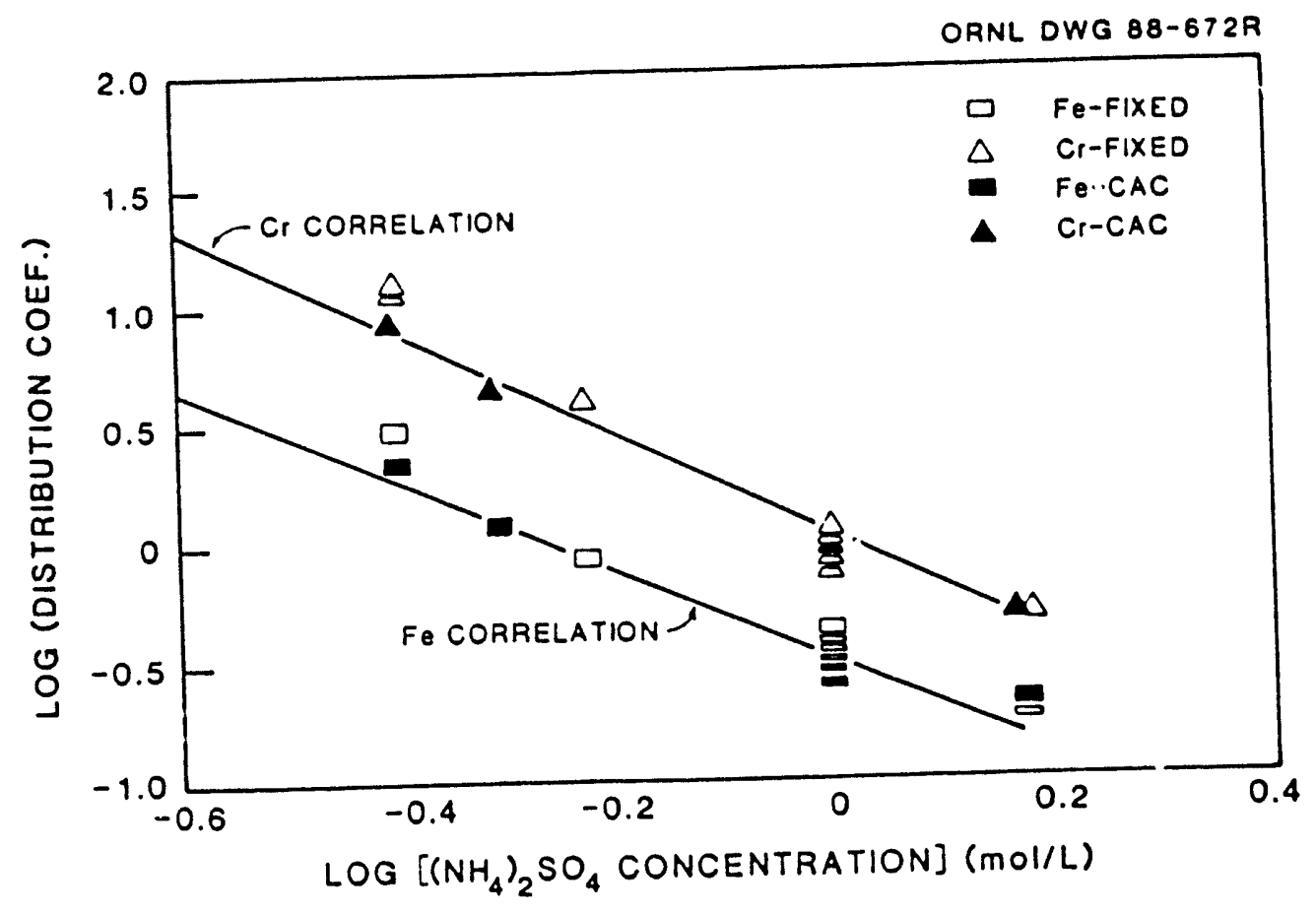

Fig. 3.3 Distribution coefficient as a function of the ammonium sulfate concentration. The eluent contains $0.025 \mathrm{M} \mathrm{H}_{2} \mathrm{SO}_{4}$. Data at $\left[\left(\mathrm{NH}_{4}\right)_{2} \mathrm{SO}_{1}\right]=1 \underline{\mathrm{M}}$ include measurements at $0.5,1.0,5.0$ and $10.0 \mathrm{~g} / \mathrm{L}$ of $\mathrm{Fe}^{3+}$ and $\mathrm{Cr}^{3+}$. 


\subsubsection{CAC Operation}

Initially the CAC was operated in the isocratic mode using a $5.0 \mathrm{~g} / \mathrm{L}$ mixture of $\mathrm{Fe}^{3+}$ and $\mathrm{Cr}^{3+}$ (ferrous and chromic nitrate dissolved in water). The eluent concentration was varied between $0.4 \mathrm{M}$ and $1.5 \mathrm{M}$ ammonium sulfate at con-

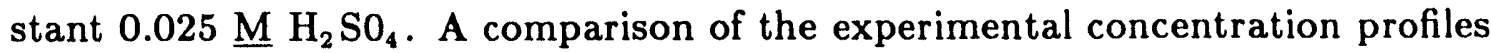
obtained for an isocratic run on the CAC and calculations based on Eq. 3.4 is shown in Fig. 3.4. The equilibrium and mass transfer parameters listed in Table 3.1 were used for these calculations. The agreement here is good, indicating that the increased effective dispersion in the CAC does not significantly affect the separation performance. Operation of the CAC with a step in eluent concentration is shown in Fig. 3.5. The beginning of the step eluent region is where the bands are sharply eluted down. Note that the bands initially begin to spread as would be expected in isocratic elution. They become sharp again, however, when subjected to an increased eluent strength. A comparison of the concentration profiles for an isocratic experiment and this stepwise elution experiment is illustrated in Fig. 3.6. Obviously, there are very significant advantages in operating with a step eluent. Not only is dilution decreased but the available bed capacity is also increased. Since the stepwise elution run requires only about $90^{\circ}$ to completely elute while the isocratic run requires the full $360^{\circ}$, the bed capacity can conceivably be increased by a factor of four by using multiple feed and step eluent nozzles. This demonstrates that by using a step eluent, the dilution can be decreased and the bed capacity increased.

Several CAC operating conditions were varied systematically and the effect on separation performance was determined. A series of stepwise elution experiments were carried out starting ai the base conditions given in Table 3.2. The eluent concentration, the rotation rate, the feed concentration, and the feed flow rate were varied from these conditions one at a time. The separation performance was expressed in terms of peak concentration and resolution. The resolution, $R$, 
Table 3.1 Equilibrium and Mass Transfer Parameters ${ }^{\ddagger}$

\begin{tabular}{cccc}
\hline Species & $\alpha^{\dagger}$ & $\beta^{\dagger}$ & $k^{\prime}, \min ^{-1}$ \\
\hline $\mathrm{Fe}^{3+}$ & 0.317 & -1.92 & 4.6 \\
$\mathrm{Cr}^{3+}$ & 1.06 & -2.17 & 3.0 \\
\hline
\end{tabular}

† $K=\alpha\left(C_{\mathrm{s}}\right)^{\beta}$, with $C_{\mathrm{o}}$ in $\mathrm{mol} / \mathrm{L}$

‡ eluent contains $0.025 \underline{\mathrm{M}} \mathrm{H}_{2} \mathrm{SO}_{4}$ 


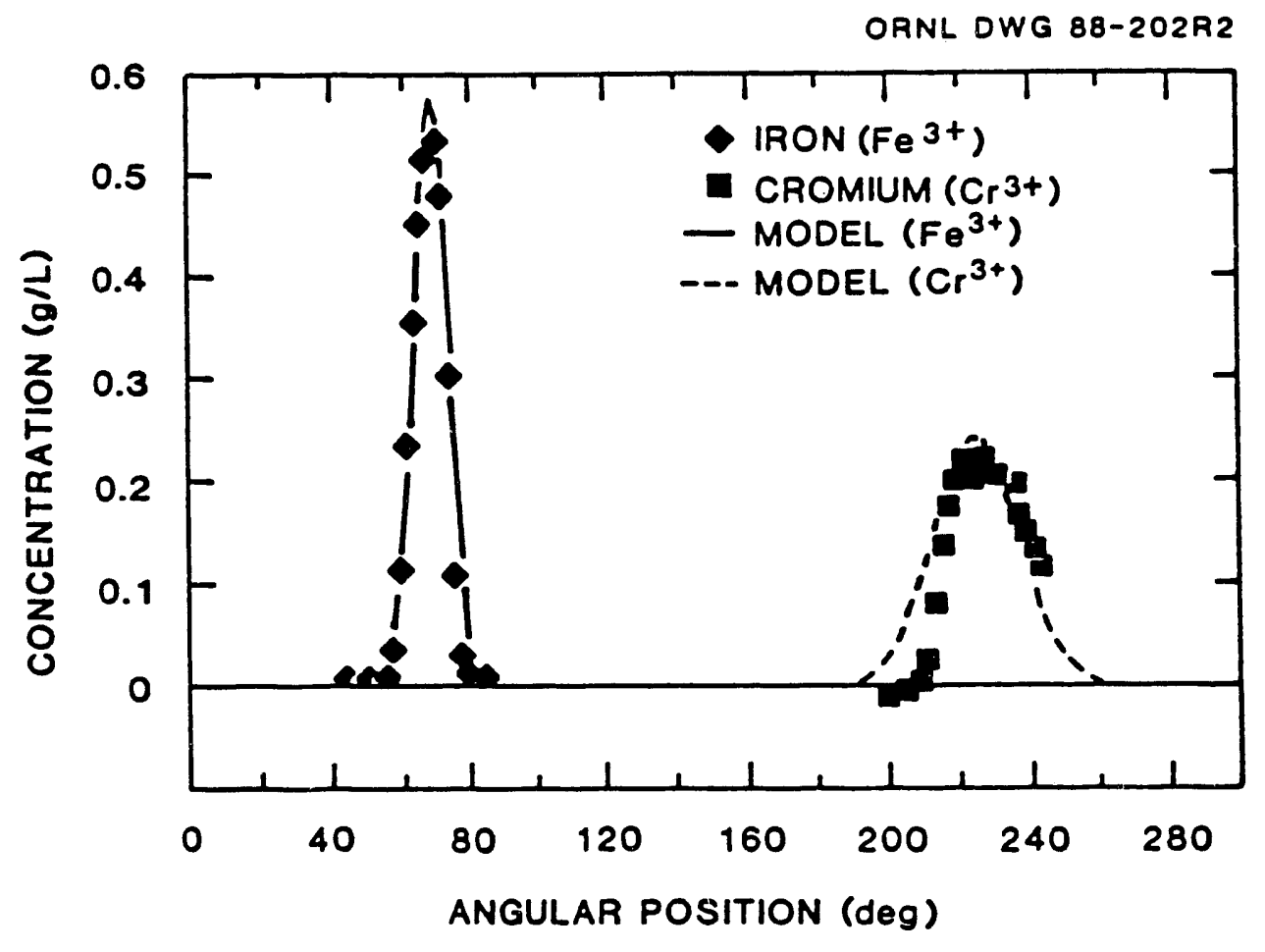

Fig. 3.4 Comparison of experimental and calculated concentration profiles for a $\mathrm{CAC}$ isocratic run, using $0.4 \underline{\mathrm{M}}\left[\mathrm{NH}_{4}\right]_{2} \mathrm{SO}_{4}$ eluent. 


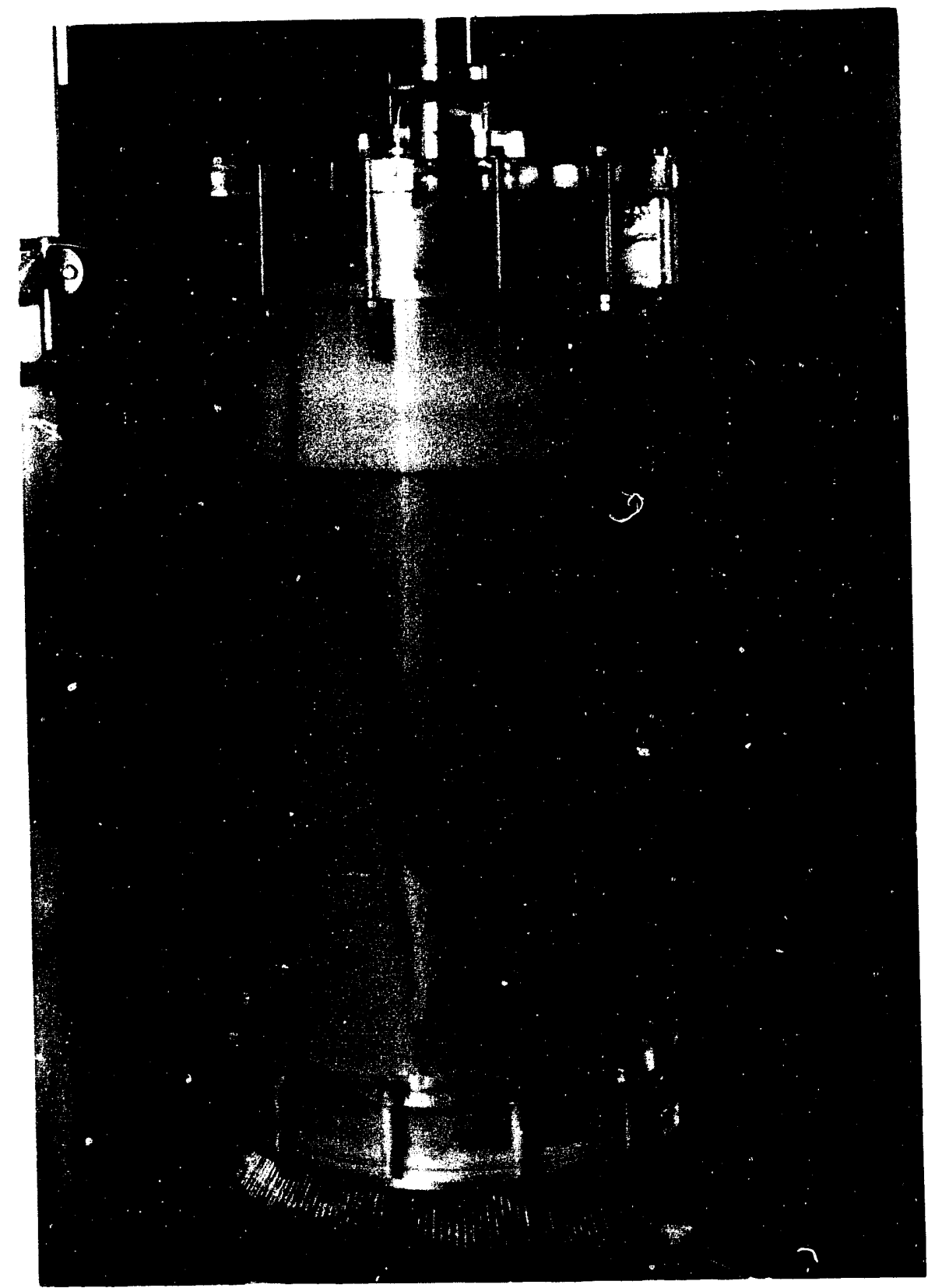

Fig. 3.5 CAC step-elution separation of a mixture of ferric and chromic nitrates. 
ORNL DWG B7-1284R4

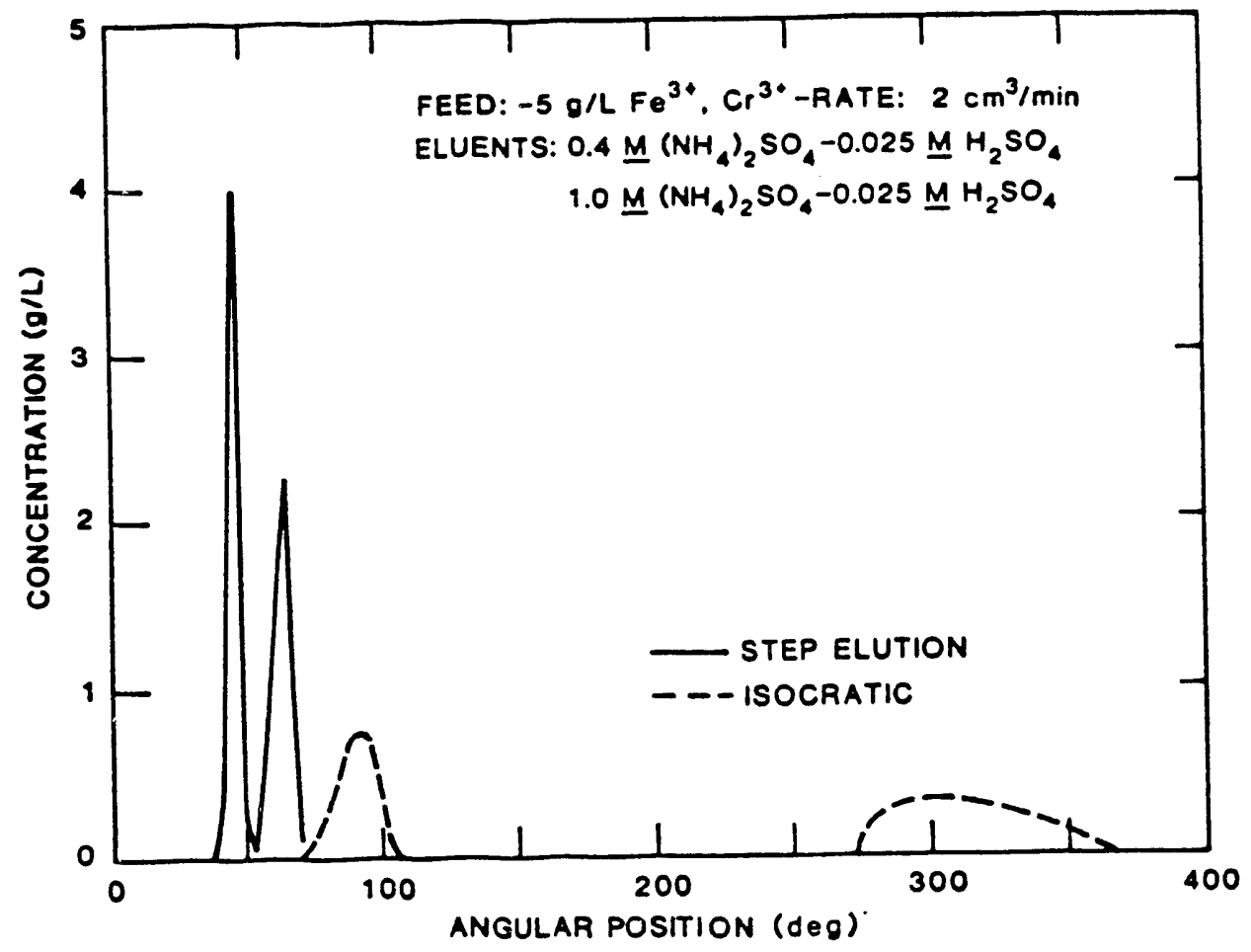

Fig. 3.6 Comparison of CAC concentration profiles obtained for isocratic and step-elution operations. 
is defined as

$$
R=\frac{2\left(\bar{\theta}_{2}-\bar{\theta}_{1}\right)}{W_{2}+W_{1}}
$$

where

$$
\bar{\theta}_{i}=\frac{\omega z}{u}\left[\epsilon+(1-\epsilon) K_{i}\right]
$$

is the peak elution angle for each species, $\mathrm{Fe}^{3+}$ and $\mathrm{Cr}^{3+}$, and $W_{i}$ is the corresponding exit band width. The band width was determined by projecting a tangent to the concentration curves at half the maximum peak height down to the baseline. The angular difference of these two intercepts with the baseline is the band width. A resolution of one indicates nearly complete separation.

The first, and perhaps most important, factor that was varied was the eluent step concentration. With a main eluent of $0.4 \underline{\mathrm{M}}$ ammonium sulfate, step eluents of $0.75,1.0$, and $1.5 \underline{\mathrm{M}}$ ammonium sulfate were investigated. Figure 3.7 illustrates the experimental and theoretical concentration profiles obtained with the CAC apparatus. The upper portion of the figure shows the iron concentrations, while the lower portion shows the chromium concentrations. This figure shows that by increasing the concentration of the step eluent, the peak elution angle, $\boldsymbol{\theta}_{i}$, decreases. This is exactly the trend that is expected since an increase in ammonium sulfate concentration causes a rapid decrease in the distribution coefficient. Accompanied with this decrease is an increase in peak concentration. By rapidly decreasing the distribution coefficient, the bands are compressed. Fig. 3.8 illustrates the overall effects of the step eluer.t concentration on peak concentration and resolution. Here, resolution decreases as the ammonium sulfate concentration decreases. From Fig. 3.8 it appears that in order to attain complete separation (i.e., $R \cong 1.0$ ) with maximum concentration, a step eluent concentration of $1.2 \underline{\mathrm{M}}$ would be required. For these conditions, the iron peak concentration would be slightly higher than its feed value, while the chromium peak concentration would be about one half of the feed value. Comparison of the theoretical and experimental results in the two figures shows relatively good agreement, especially in view of the fact that the distribution coefficients are 
Table 3.2 Base Conditions for CAC Step Elution Experiments

\begin{tabular}{cc}
\hline Parameter & Value \\
\hline Resin Bed Depth, cm & 49 \\
Glass Bead Depth, cm & 14 \\
Main Eluent ${ }^{\dagger}$ Flow Rate, $\mathrm{cm}^{3} / \mathrm{min}$ & 170 \\
Step Eluent & 25 \\
Step Elow Rate, $\mathrm{cm}^{3} / \mathrm{min}$ & 18 \\
Rotation Rate, deg $/ \mathrm{h}_{\text {Feed }}^{*}$ Rate, $\mathrm{cm}^{3} / \mathrm{min}$ & 90 \\
\hline
\end{tabular}

+ Main Eluent - 0.4 $\underline{\mathrm{M}}\left[\mathrm{NH}_{4}\right]_{2} \mathrm{SO}_{4}$ containing $0.025 \underline{\mathrm{M} \mathrm{H}} \mathrm{HO}_{4}$.

t Step Eluent $-0.4 \underline{\mathrm{M}}\left[\mathrm{NH}_{4}\right]_{2} \mathrm{SO}_{4}$ containing $0.025 \underline{\mathrm{M}} \mathrm{H}_{2} \mathrm{SO}_{4}$.

* Feed $-5 \mathrm{~g} / \mathrm{L} \mathrm{Fe}^{3+}$ and $5 \mathrm{~g} / \mathrm{L} \mathrm{Cr}^{3+}$ as nitrate in water. 


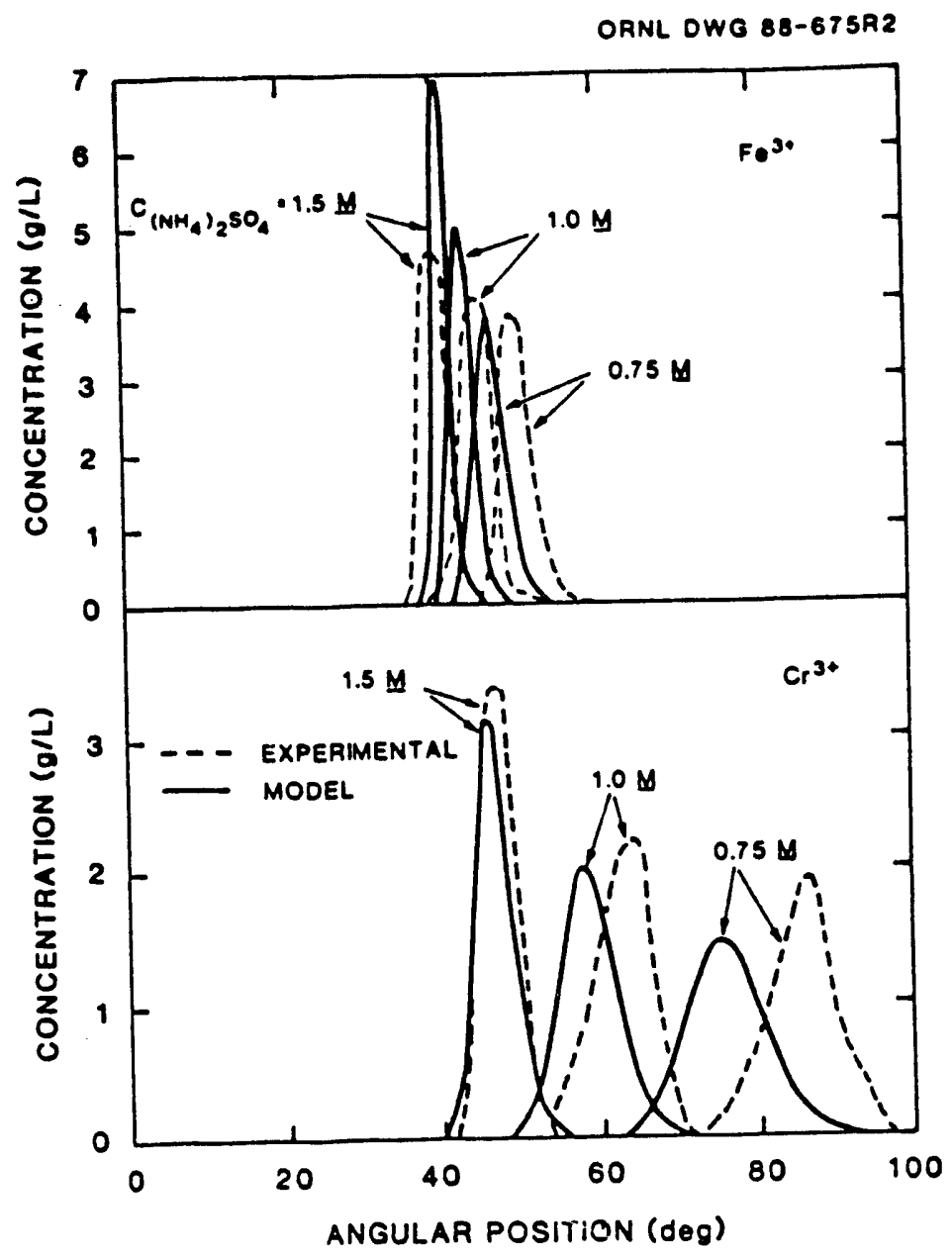

Fig. 3.7 Effect of step-eluent concentration on CAC separation at base conditions (Table 3.2). Comparison of model and experimental results. 
so strongly dependent on the eluent concentration that small errors in the prediction of this concentration have a large effect on the predicted concentration profiles.

The effects of rotation rate were also investigated and experimental and model calculations are shown in Figs. 3.9 and 3.10. From the experimental concentration profiles in Fig. 3.9, it is evident that increasing the rotation rate increases the peak elution angle and decreases the peak concentrations.

This becomes even more apparent in Fig. 3.11, which shows the prediction of this effect for rotations rates lower than those investigated experimentally. If there is no rotation the feed components exit the column essentially at the same concentration at which they entered, $5.0 \mathrm{~g} / \mathrm{L}$. As the rotation is increased, the peak concentrations will either be constant until rotation is fast enough that the step eluent has an effect or the concentrations will begin to decrease as isocratic dilution effects become important. For chromium, isocratic dilution effects are not important in the main eluent, as evidenced by the horizontal line from 0 to $10^{\circ} / \mathrm{h}$ on the rotation rate axis. The iron peak concentration, however, shows a dip at around $30^{\circ} / \mathrm{h}$ that is due to isocratic dilution effects of the main eluent. As the rotation rate was increased, there was a sharp increase in peak concentration for both components. (At this point they had been subjected to the step eluent, which caused the sharp rise in peak concentration.)

Since the step eluent position is fixed, an increase in rotation rate caused the iron and chromium bands to be affected by the step eluent nearer the top of the bed. Once the bands had been subjected to the step eluent and had been compressed, they traveled the rcmaining length of the column in the isocratic mode with the step eluent behaving isocratically. Also, the closer to the top of the column the bands were subjected to the step eluent, the more column length was available for isocratic elution within the step eluent to occur. Since isocratic elution is essentially a dispersive technique, the longer the band is subjected to isocratic effects within the step eluent, the more dilute they should become. Although experiments to verify the position of this sharp increase were 


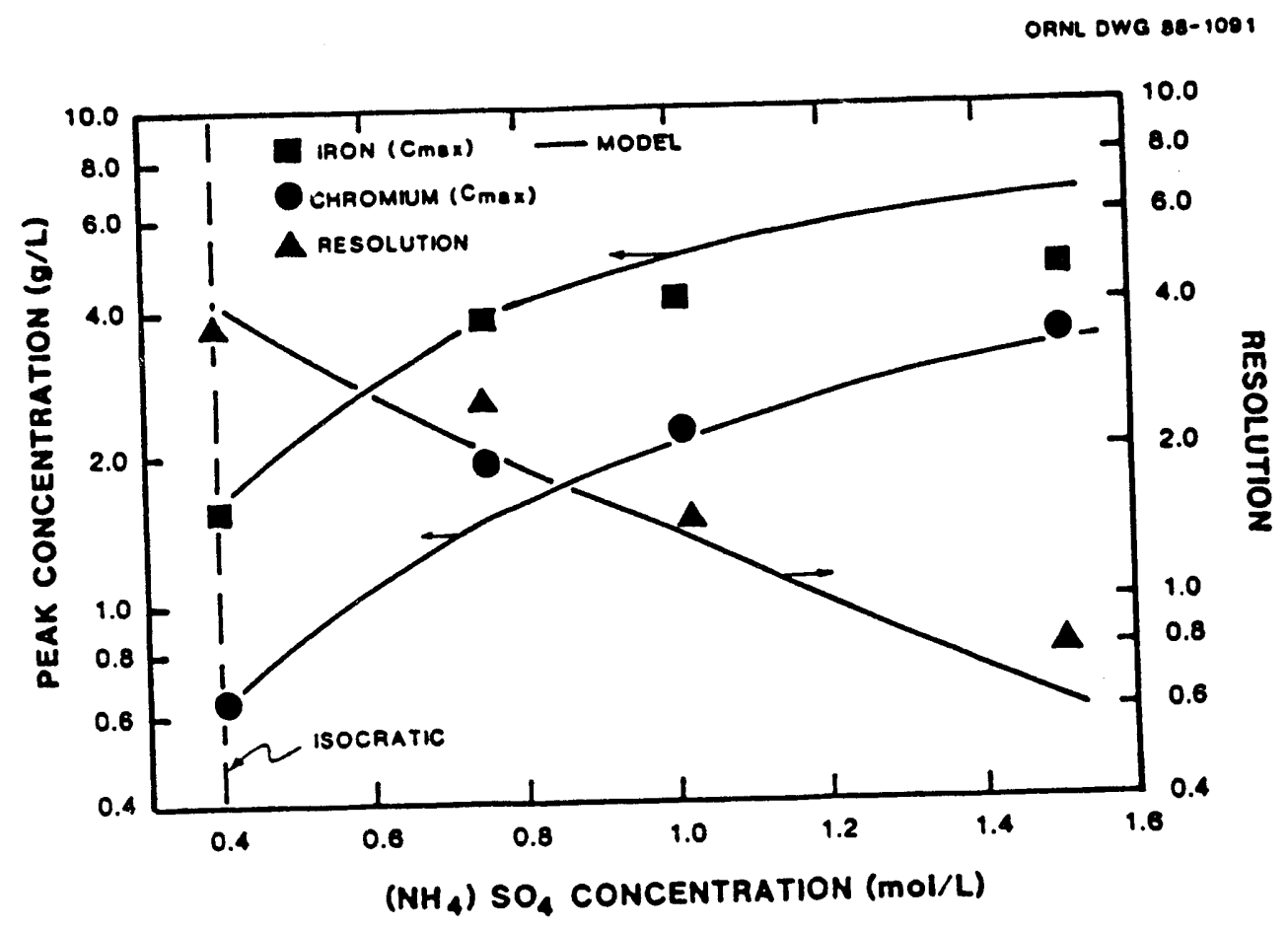

Fig. 3.8 Peak concentrations and resolution obtained by varying the step eluent concentration at base conditions (Table 3.2). 


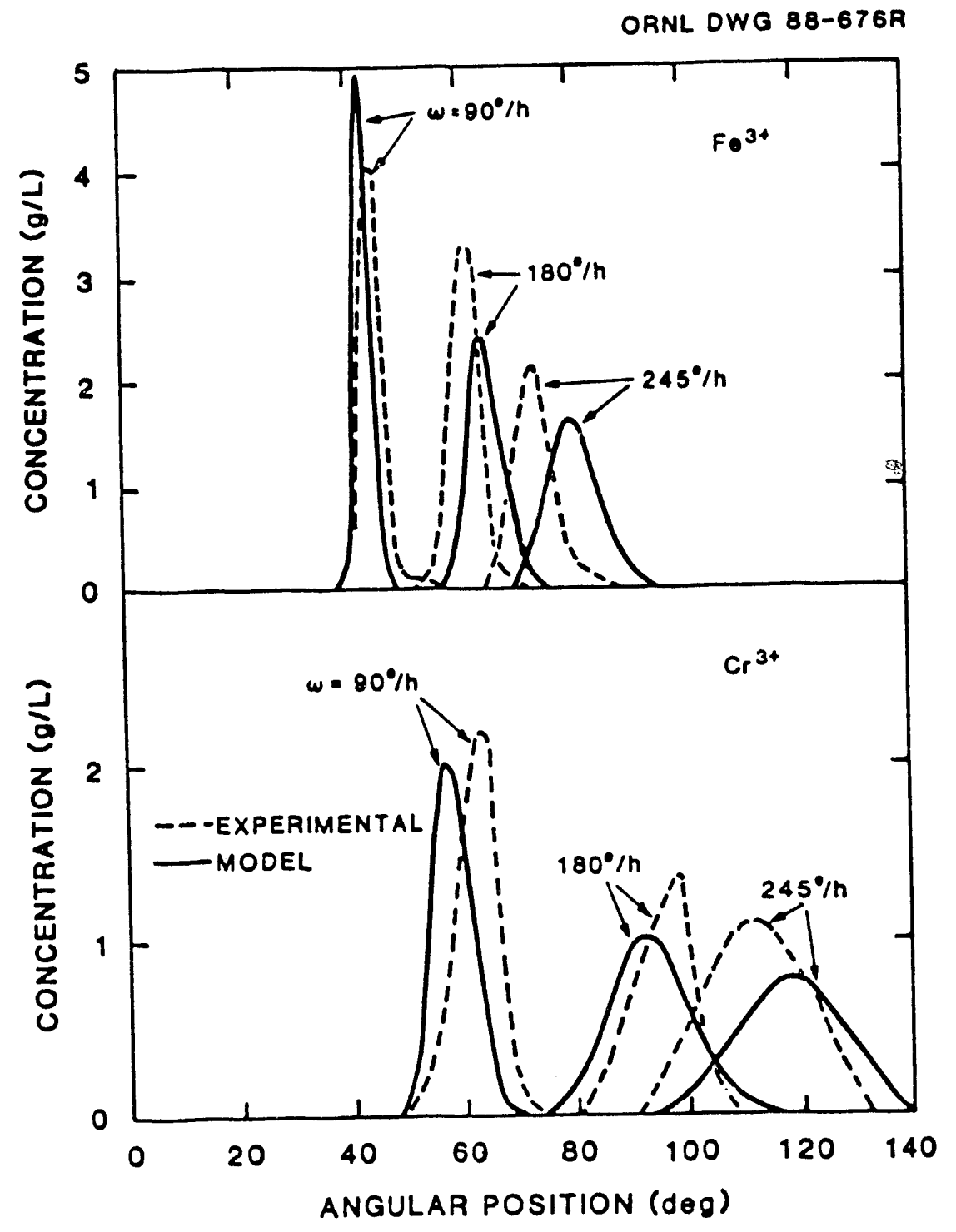

Fig. 3.9 Effect of rotation rate on CAC separation at base conditions (Table 3.2). Comparison of model and experimental results. 
ORNL DWG 88-1092R

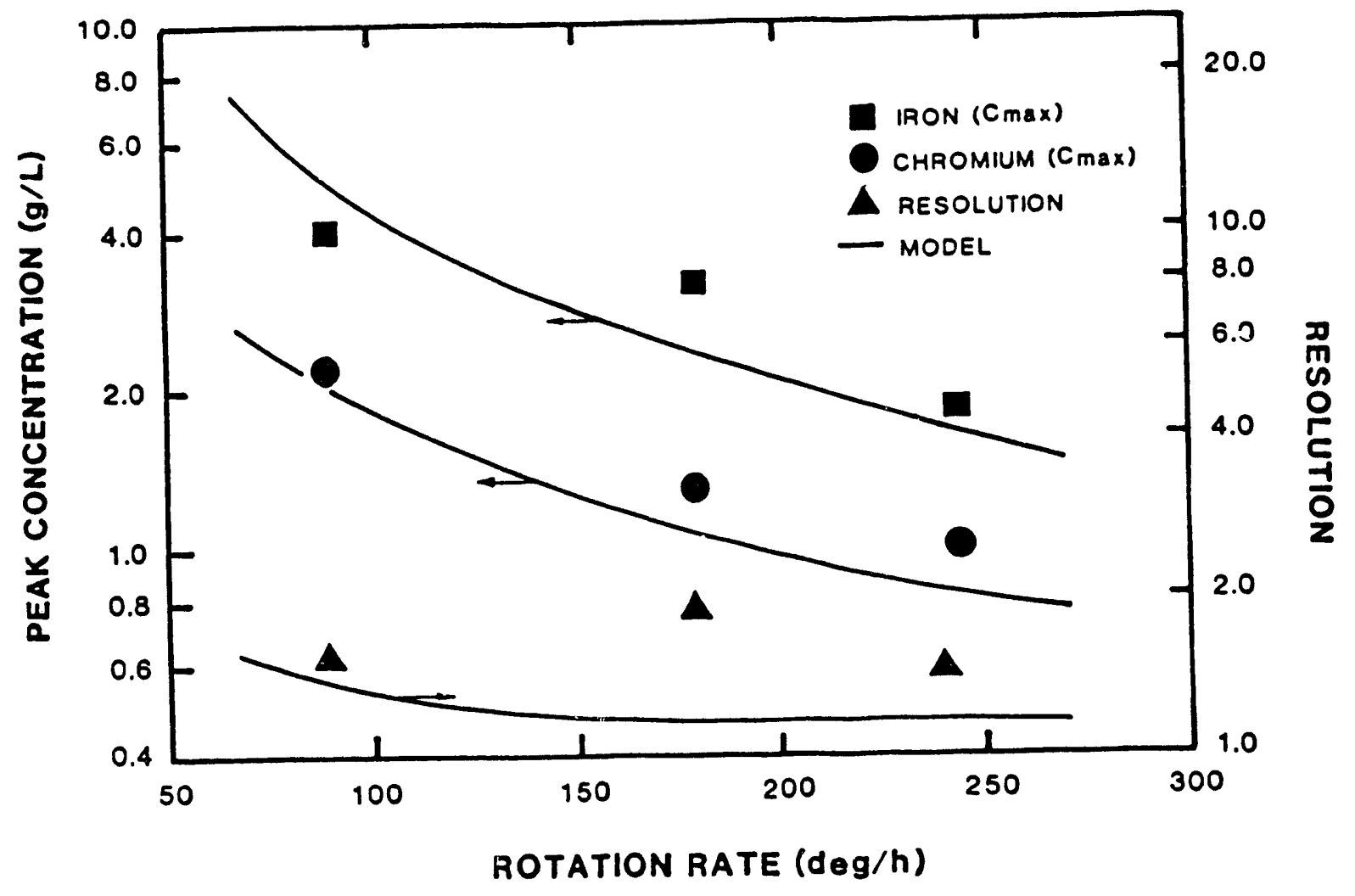

Fig. 3.10 Peak concentrations and resolution obtained by varying the rotation rate at base conditions (Table 3.2 ). 
ORNL DWG 88-1093

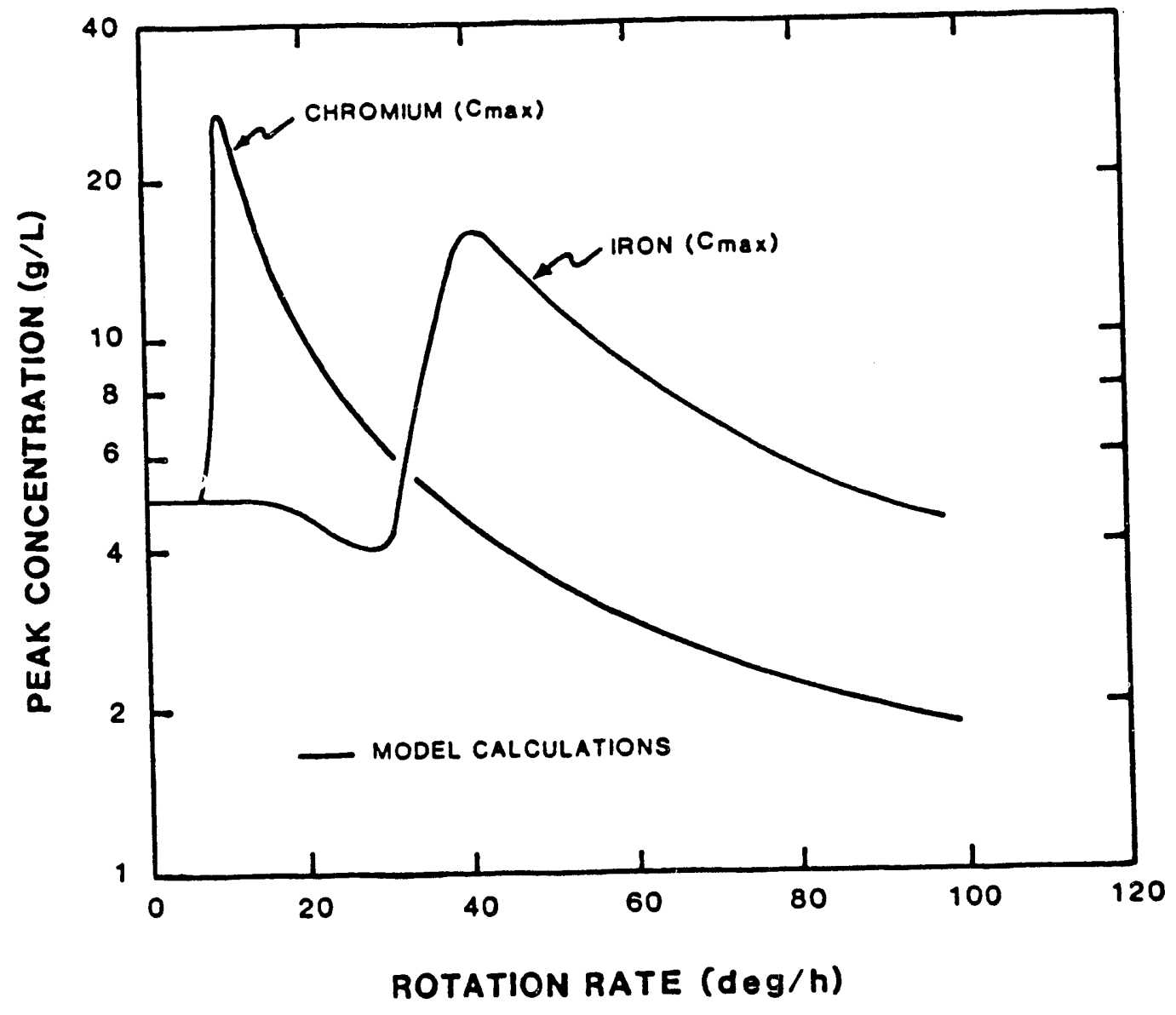

Fig. 3.11 Calculated effect of rotation rate on peak concentrations obtained at base conditions (Table 3.2). 
not made at very low rotations, experimental results do agree with the model predictions for rotation rates studied.

The effect of feed loading was also studied by varying the feed concentration and the feed flow rate. Peak concentration and resolution for these two conditions are given in Figs. 3.12 and 3.13, respectively. In Fig. 3.12, the resolution is essentially independent of the feed concentration for the model and is relatively so for the experimental results. Increasing feed concentration caused an essentially linear increase in product concentration since the equilibrium was basically linear. However, the maximum peak concentration in the experiments and the model predictions indicated that the peak concentration would not be any higher than that of the feed concentration. Therefore, by increasing the feed concentration, the numerical value of the peak concentration increased but the dilution of the peak appeared to remain the same.

Figure 3.13 shows the effects of increasing the feed flow rate on resolution and peak concentration. In these experiments, increasing the feed flow rate increased the peak concentration. Since the feed concentrations in these experiments were const:ant, increasing the feed flow rate tended to decrease the effects of dilution. in fact, as is shown here, the peak concentrations are actually much greater than the feed concentrations. This is more pronounced for iron because this species has a lower distribution coefficient. In addition, there was a slight decrease in the resolution with a higher flow rate.

Finally, an experiment was conducted in which the feed nozzle position was changed from $35^{\circ}$ to $90^{\circ}$. At the same time the feed concentration of both metals was decreased to $1.0 \mathrm{~g} / \mathrm{L}$, the feed flow rate was increased to $10 \mathrm{~cm}^{3} / \mathrm{min}$, and the rotation rate was increased to $180^{\circ} / \mathrm{h}$. The concentration profile and the model prediction for this experiment are shown in Fig. 3.14. Here, the model underestimates the peak concentrations for both the iron and chromium peaks. Most likely this is due to slight differences in the eluent concentrations between model and experiment since the distribution coefficients are so sensitive to the eluent concentrations. Experimentally, near-complete separation was attained, 
ORNL DWG 88-1090

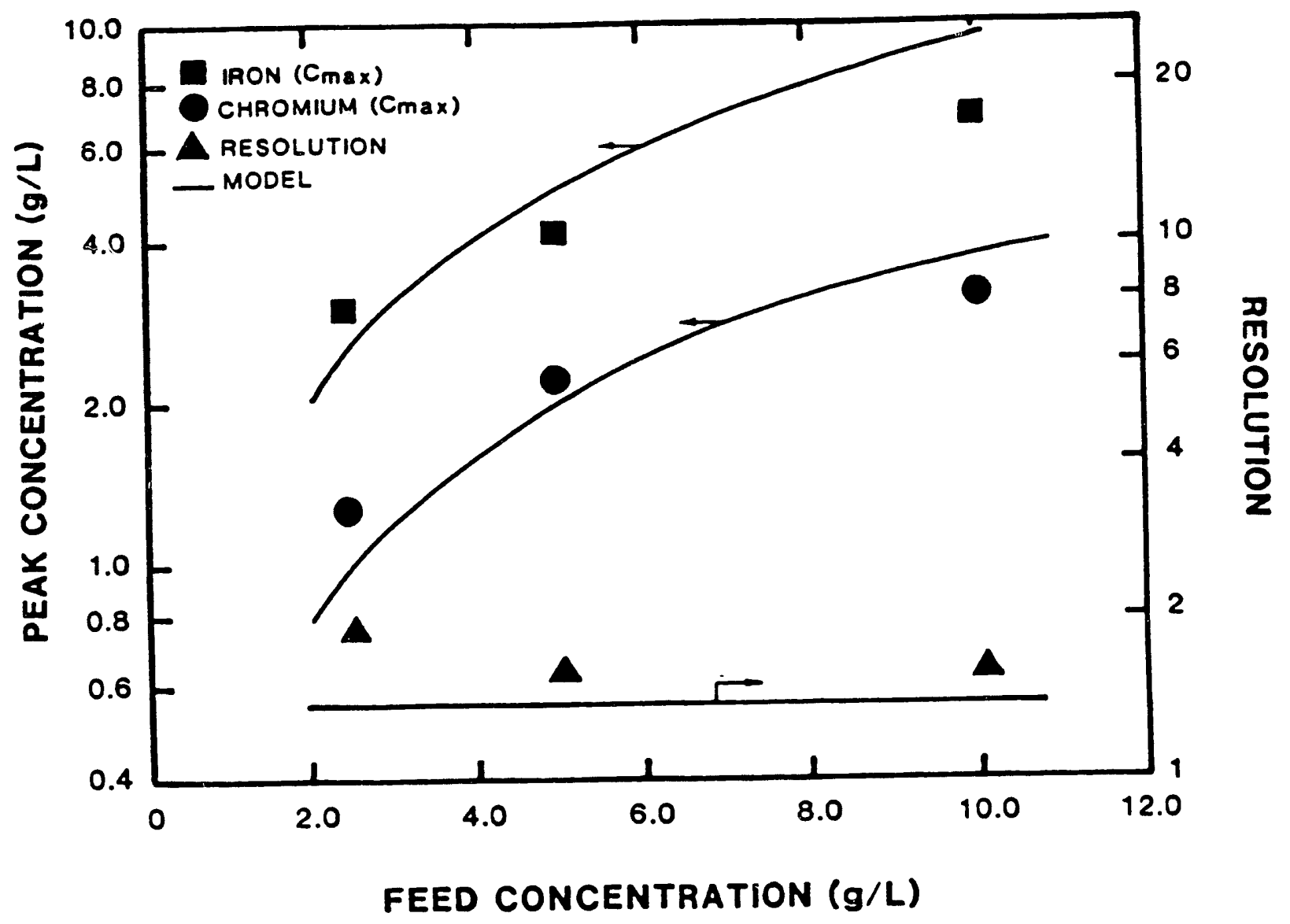

Fig. 3.12 Peak concentrations and resolution obtained by varying the feed concentration at base conditions (Table 3.2). 
ORNL DWG 88-1089

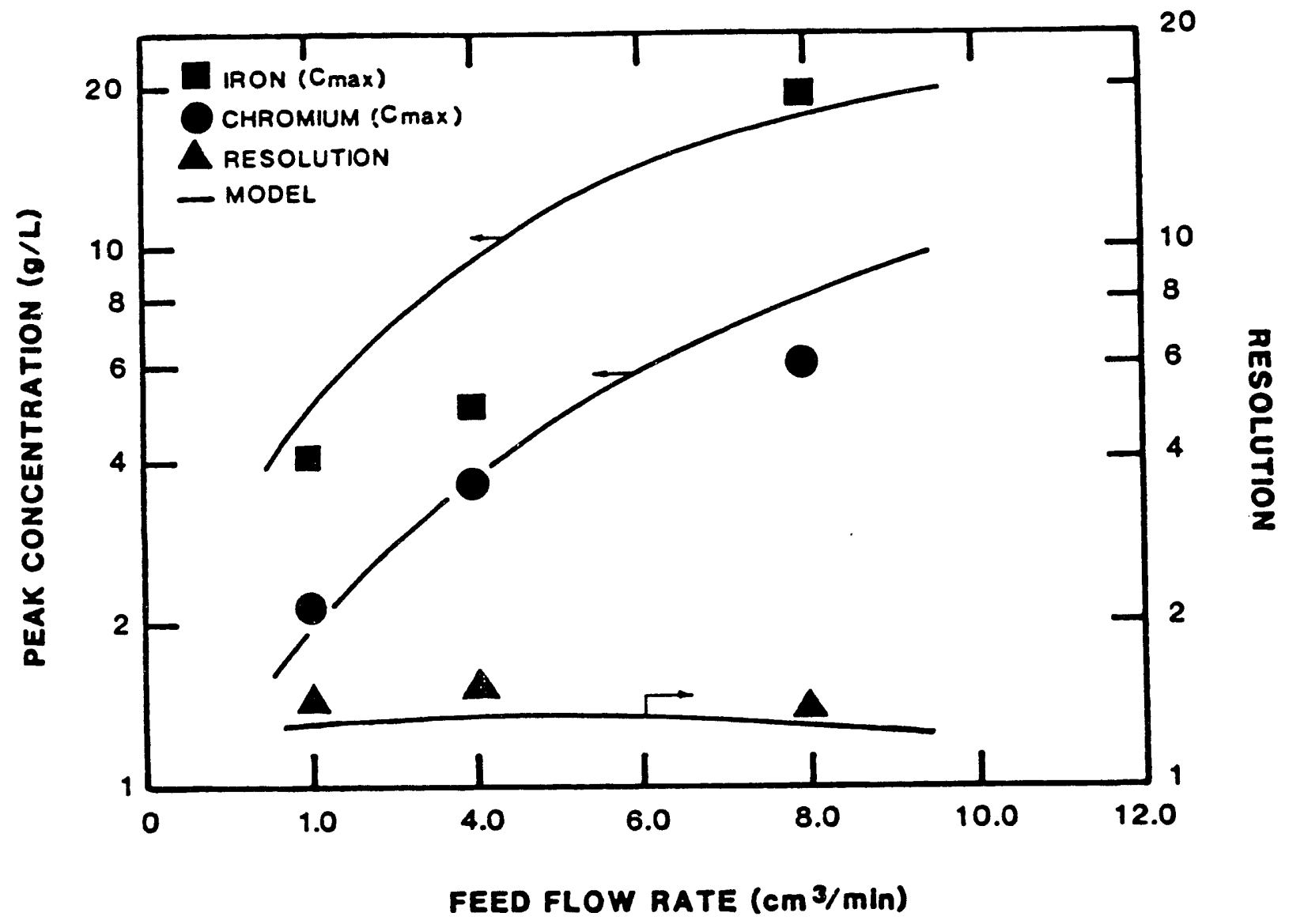

Fig. 3.13 Peak concentrations and resolution obtained by varying the feed flow rate at base conditions (Table 3.2 ). 


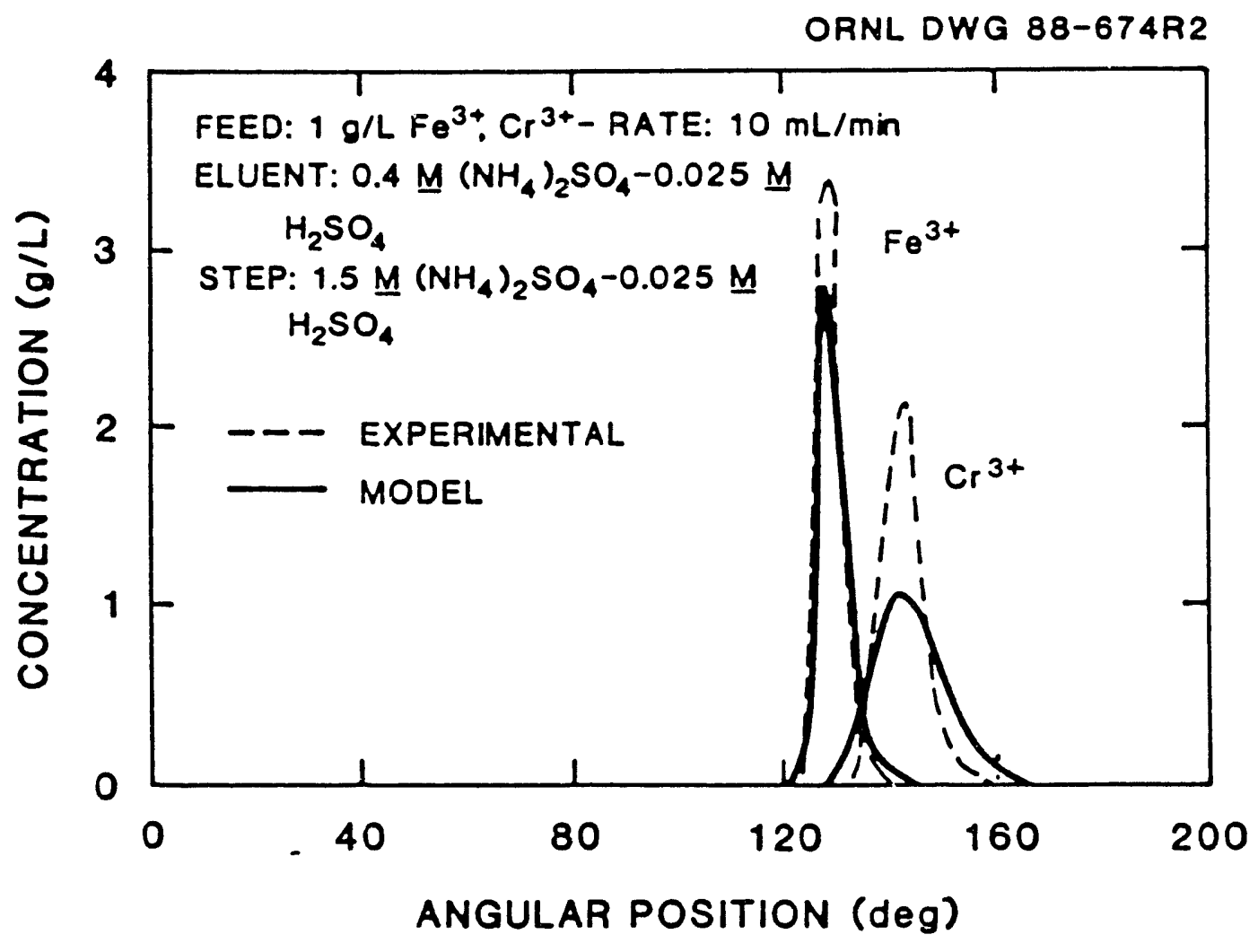

Fig. 3.14 Concentration profiles obtained in step-elution CAC separation of a dilute mixture of ferric and chromic nitrate. 
but the peak concentrations were greater than twice the feed concentration. When the average concentrations for this experiment were calculated for iron and chromium from the experimental data, they were found to be 1.4 and $1.0 \mathrm{~g} / \mathrm{L}$, respectively. The separation, therefore, was accomplished with almost complete resolution, a $40 \%$ concentration of iron, and no dilution of chromium. It appears that ideal conditions were those where the components were separated in the low concentration eluent, quickly eluted from the resin with the high concentration eluent, and then immediately exited the column to avoid dispersive effects within the step eluent.

\subsection{SUMMARY AND CONCLUSIONS}

Experimental results and model calculations for the separation of mixtures of iron and chromium ions with an acid ammonium sulfate eluent were obtained. Linear equilibrium in the range 0 to $10 \mathrm{~g} / \mathrm{L}$ for iron and chromium was experimentally verified. Fixed-bed experiments were used to determine the equilibrium distribution coefficient, which was found to be a strong function of eluent concentration. The higher the eluent concentration, the lower the equilibrium distribution coefficient. A model assuming linear solid-film mass transfer resistance was found adequate to describe the system. The model can be used to design step-elution experiments.

The CAC apparatus was modified with provisions for introducing a step in eluent concentration. Both isocratic and stepwise elution experiments were carried out. It was confirmed that by using a step eluent, dilution could be decreased and bed capacity increased. At certain operating conditions, dilution could be eliminated, with the possibility of achieving continuous separation along with product concentration. In addition, all of these effects were observed with essentially complete separation of the components of a feed stream (i.e., resolution $\geq 1)$. 
It should also be possible to extend this method to gradient elution, where a gradual change of eluent concentration is used instead of an abrupt one. Possible implementation might include a series of step eluent nozzles, each with a slightly higher eluent concentration. Although different in practice from step elution, the principle is the same. By using gradient and stepwise elution it should not only be possible to improve on previous isocratic separations, but also to expand the applicability of the CAC to systems that can only be separated by these advanced elution techniques. This work, therefore, exposes CAC to an entirely new range of applications. 


\section{SEPARATION OF AMINO ACIDS BY DISPLACEMENT DEVELOPMENT}

\subsection{INTRODUCTION}

Amino acids are among the largest volume products in biotechnology, with worldwide production of approximately $\$ 2$ billion. Amino acids are the building blocks of proteins and are essential for all living cells to carry out protein synthesis. In the food industry, they are used as a supplement for protein-poor diets and as a flavor enhancer. In the medical industry, amino acid salts are used to carry important metal ions such as potassium, magnesium, and iron into the body. In general, amino acids are used predominately for nutritional and therapeutic uses.

Amino acids originally were recovered by isolation from protein hydrolysates, but today they are produced by both chemical and microbial synthesis resulting in higher quality, purer products. Their purification is important because amino acids for direct human use must be a minimum of $98 \%$ pure and free from pyrogens. Pyrogen-free amino acids are easier to produce chemically and microbially than by isolation from proteins (Yamamoto, 1978; Reisman, 1977). The synthesis scheme for the production of an amino acid depends on which amino acid is being produced. For example, L-glutamic acid, L-valine, and L-leucine are produced by fermentation. L-alanine, however, is produced enzymatically from aspartic acid in industry because only a racemic mixture can be produced by fermentation, and it is cheaper to produce it chemically than to separate its $\mathrm{D}$ and $\mathrm{L}$ isomers. Generally speaking, biological transformations are predominately used to produce amino acids.

Once produced, the amino acid must be isolated. The steps taken in a general amino acid purification scheme are shown in Fig. 4.1. Depending on the process, one or more of these steps could be bypassed (Reisman, 1977).

Figure 4.1 illustrates that once the fermentor broth has been harvested and 
ORNL DWG 89A-566

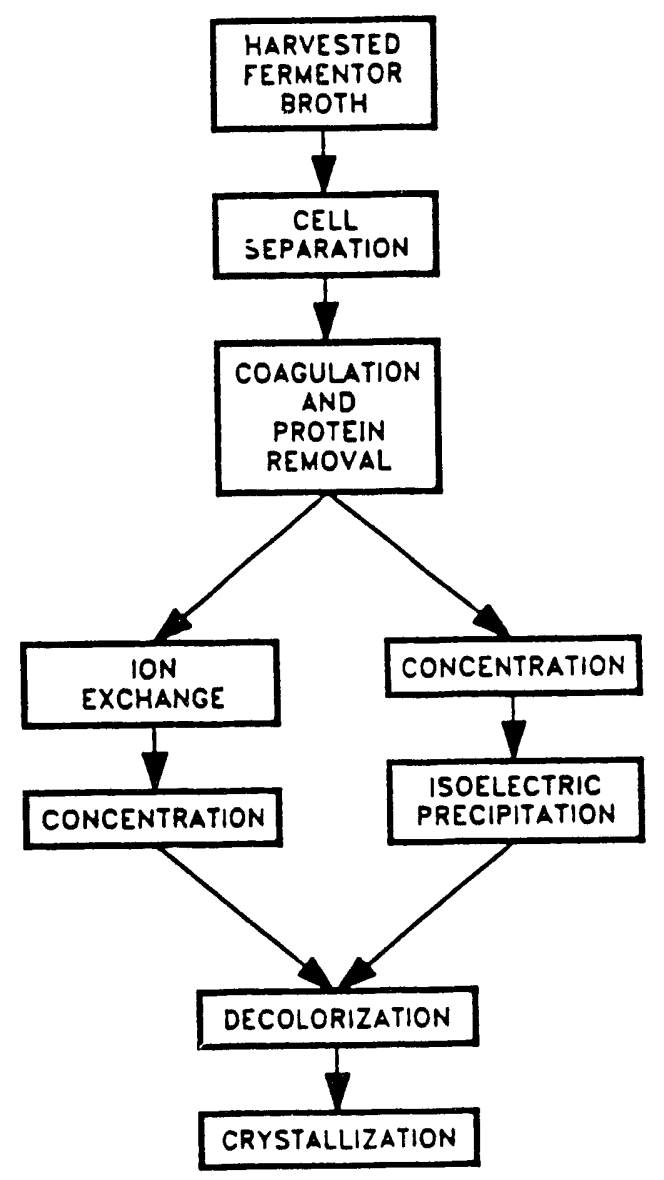

Fig. 4.1. General steps of an amino acid purification scheme (Reisman, 1977). 
the cells and other protein separated, the main purification and recovery steps are combinations of ion exchange and crystallization. This work will focus on the development of a continuous ion-exchange process. The purpose is to develop displacement chromatography on the the CAC to continuously separate and concentrate amino acid mixtures. Representative amino acids are chosen for the experimental studies as examples of potential industrial applications.

\subsection{SEPARATION OF AMINO ACIDS BY ION EXCHANGE}

Analytical scale separations of amino acids can be obtained using both ionexchange and reversed phase materials, and there is a large body of information on different separation schemes using these materials (Murren et al., 1975; Hughes and Wilson, 1982; Kulkarni and Whiteley, 1975; Young and Yamamoto, 1973; Redman, 1975; Robinson, 1975; Ganno et al., 1985; and Blackburn, 1983). Most of these methods use a variety of buffers in conjunction with solvent programming techniques, making them expensive and difficult to scaleup. In addition, they almost always use some form of elution chromatography and suffer from the inherent drawbacks of dilution and low column capacity. Displacement chromatography, on the other hand, can simultaneous separate and concentrate multicomponent mixtures and is ideal for large-scale industrial separations. There is a wealth of information on amino acid elution chromatography, but there is very little available on their separation by displacement development. One exception is a series of articles by Partridge and coworkers (1947-52). His work focused on the use of both cation- and anion-exchange resins for the separation of protein hydrolysates, and the effects of displacer composition, resin cross-linking, and temperature were also studied. Resins with $4 \%$ crosslinking exhibited the best separation performance. A 5\%-cross-linked sample showed decreased separation, while a $10 \%$-cross-linked resin was unable to separate amino acid mixtures. Increased amounts of cross-linking decrease the moisture content 
and the resin's ability to shrink and swell. In the $4 \%$-cross-linked sample, the resin shrank while feeding displacer, which subsequently caused some mixing between the amino acid bands. This effect was diminished with higher cross-linked resins. Unfortunately, the separation performance decreased or even became nonexistent. There is a tradeoff, therefore, between the amount of cross-linking al. 1 the rigidity desired (Partridge, 1950).

The effects of eluent composition in ion-exchange chromatography of amino acids are complex and strongly depend on the $\mathrm{pH}$. Amino acids can exist as both positively and negatively charged ions. At low pH they are positively charged and at high $\mathrm{pH}$ they are negatively charged. By using a strongly basic solution for the displacer, amino acids become negatively charged in the column and are excluded from cation-exchange resins ('Partridge, 1949). This effect can be exploited to carry out displacement development separations.

In this mode of operation, a large sample of a dilute feed is loaded onto a column and a strongly basic solution is used to displace it. As tire $\mathrm{pH}$ front moves down the column, the amino acids in contact with it become negatively charged and are excluded from the resin. The local $\mathrm{pH}$ of the amino acid downstream of the front depends not on the buffer or the displacer but on the sample composition itself. Partridge, for example, found that some amino acids could only be separated in the presence of others. He also found that by using a solution of moderate $\mathrm{pH}$, the separations became more like those found in the elution techniques, and it became very difficult to remove strongly basic amino acids (Partridge, 1949).

Generally speaking, an increased temperature improves amino acid separations. However, in some cases, an increase in temperature actually changed the order of elution of the amino acids from the column.

Summarizing, amino acids can exist as both positively and negatively charged ions and can be separated by ion-exchange. For displacement chromatography using cation-exchange resins, it appears that solutions with a high $\mathrm{pH}$ will be necessary and that a compromise between resin cross-linking and shrinkage ef- 
fects must be made to maximize separation.

\subsection{EQUIPMENT AND EXPERIMENTAL METHODS}

\subsubsection{Materials}

The resin used in this study was Dowex 50W-X8. The properties of this resin were described in Sect. 2.2. The degree of cross-linking of this resin appeared to be a reasonable compromise of selectivity and rigidity as was previously demonstrated in the sugar and metal-ion separation studies. A particle diameter of 30 to $60 \mu \mathrm{m}$ was r.sed. The total ion-exchange capacity of the resin was determined by equilibrating a sample of the resin in hydrogen form with a volume of solution containing an excess of $\mathrm{NaOH}$. At equilibrium, the $\mathrm{NaOH}$ excess was titrated with $\mathrm{HCl}$ and the capacity determined from a material balance. Using this technique, the resin capacity was found to be $5.6 \pm 0.2 \mathrm{meq} / \mathrm{g}$ dry resin (Dye, 1988).

A determination of the dry weight of the resin was also necessary because that the ionic form of the resin will change during the displacement process. Correspondingly, there will be volume changes which render a definition of equilibrium uptake in volumetric terms impractical. Using the dry weight as a reference, one defines instead a unique, reproducible measurement of equilibrium uptake. To determine the dry weight, a fully hydrated sample of the resin in the hydrogen form was "filtered" in a buchner funnel to remove the interstitial water and then dried at $110^{\circ} \mathrm{C}$. The dry weight ( $\mathrm{g}$ dry resin/g wet resin) was finally determined from the weight loss of the sample. Using this technique, the dry weight of the hydrogen form of the resin was found to be $0.45 \pm 0.03 \mathrm{~g} \mathrm{dry} \mathrm{resin/g} \mathrm{wet} \mathrm{resin.}$ This value is consistent with values reported in literature for resins with similar degrees of cross-linking and capacity (Helfferich, 1962).

The amino acids L-glutamic acid, L-valine, and L-leucine were selected as a model system. Their relevant physicochemical properties are given in Tables 
4.1 and 4.2. As seen in Table 4.1, the three amino acids are rather soluble. In Table 4.2, glutamic acid has a low isoelectric $\mathrm{pH}$, while valine and leucine have approximately the same value. The latter two amino acids differ in their hydrophobic character. A mixture of the three amino acids, thus, provides a good example of two types of separations. The separation of glutamic acid from valine and leucine is expected to be derived primarily from the large isoelectric pH difference. The separation of valine and leucine can, instead, only occur by virtue of differences in the specific interactions of the amino acid cations with the resin.

The three amino acids were obtained with purity greater than $99 \%$ from Sigma and Ajinomoto USA, Inc., and were used without further purification. Other chemicals were obtained from Fisher and were analytical reagent grade.

The concentrations of amino acid in aqueous solutions were determined by HPLC using the technique described by Dye (1988).

\subsubsection{Equilibrium Uptake}

The determinations of the equilibrium uptake of the amino acids by the resin were performed in shaker flasks. For these experiments, samples of the resin in the hy irogen form were placed in flasks in contact with a volume of solution containing amino acids of known concentration. The mixture was then shaken in a constant-temperature bath at $25 \pm 1^{\circ} \mathrm{C}$ for 12 to $24 \mathrm{~h}$. A material balance on the amino acid can be represented by the following equation:

$$
q_{A} M=\left(C_{A}^{\circ}-C_{A}^{*}\right) V
$$

where $q_{A}$ is the amount of amino acid taken up by the resin (mmol/g dry resin), $C_{A}^{\circ}$ is the initial amino acid concentration (mmole/L), $M$ is the mass of resin (g dry resin), $V$ is the volume of solution added $(\mathrm{L})$, and $C_{A}^{*}$ is the equilibrium solution concentration of amino acid (mmole/L). $C_{A}^{*}$ was determined by HPLC 
Table 4.1 Solution Equilibrium Paramete"s

\begin{tabular}{lllll}
\hline & $p K_{1}$ & $p K_{2}$ & $p K_{3}$ & $p I$ \\
\hline L-GLU & 2.19 & 4.25 & 9.67 & 3.22 \\
L-VAL & 2.32 & 9.62 & - & 5.97 \\
L-LEU & 2.36 & 9.62 & - & 5.99 \\
\hline
\end{tabular}

Table 4.2 Solubility of Amino Acids in Water at their Isoelectric $\mathrm{pH}$

\begin{tabular}{lll}
\hline Amino Acid & $\begin{array}{l}\text { Solubility in Water at } 25^{\circ} \mathrm{C} \\
\mathrm{g} / \mathrm{L}\end{array}$ & $\mathrm{mM}$ \\
\hline L-Glutamic acid & $8.64^{\dagger}$ & 58.7 \\
L-Valine & $88.5^{\dagger}$ & 755 \\
L-Leucine & $24.2^{\dagger}$ & 184 \\
\hline
\end{tabular}

${ }^{\dagger}$ CRC Handbook of Chemistry and Physics (1977) 
and all experiments were designed to give a significant change $(\sim 50 \%)$ in the solution concentration. The procedure is more fully described by Dye (1988).

Uptake equilibrium experiments were performed with the hydrogen form of resin for L-glutamic acid, L-valine, and L-leucine. Various sets of uptake measurements for different solution $\mathrm{pH}$ values were obtained by adding varying amounts of $\mathrm{HCl}$ to the shaker flasks. The coion, $\mathrm{Cl}^{-}$, is excluded from the resin by the Donnan potential effect and remains essentially constant during the exchange process. The hydrogen ion concentration (or the $\mathrm{pH}$ ) will vary during the exchange process as $\mathrm{H}^{+}$ions are released in stoichiometric proportion. Thus, trying to maintain constant $\mathrm{pH}$, proves to be a very difficult task unless high buffer concentrations are used. In this case, however, extraneous counterions are introduced with the buffer and the resin capacity remains underutilized. Performing the experiments at a constant coion concentration eliminates these problems. The solution $\mathrm{pH}$ can easily be measured or calculated (as is discussed in Sect. 4.4.1).

Finally, a few shaker flask experiments were conducted by placing resin, which was initially fully loaded, with the amino acids that were in contact with solutions containing an excess of $\mathrm{NaOH}$. Once in contact with these solutions, the amino acids became negatively charged and were excluded from the resin. The final equilibrium uptake of amino acid was found to be essentially zero. This confirmed that $\mathrm{NaOH}$ solutions can be used as effective displacers since they will remove amino acids from the resin completely.

\subsubsection{Fixed-Bed and CAC Experiments}

The fixed-bed experiments were performed in an apparatus similar to the one used to determine equilibrium and mass transfer parameters for the metal ion system described in Sect. 3.2. The resin was packed in a glass column $1.45 \mathrm{~cm}$ in diameter, with a maximum packing height of $20 \mathrm{~cm}$. Amino acids were dissolved 
in deionized distilled water and loaded onto the column with a microprocessorcontrolled Waters 501 HPLC pump. The displacer, a $\mathrm{NaOH}$ solution, was fed directly after the amino acids. Samples of the effluent at the column outlet were collected with a Gilson Model 231 fraction collector.

The $\mathrm{pH}$ of each sample was determined with a $\mathrm{pH}$ probe and the concentration of amino acid by HPLC analysis. At the start of each displacement experiment the resin was in the hydrogen form. By feeding the displacer, the resin was converted to the sodium form and it was necessary to convert the resin back to the hydrogen form before another displacement experiment could be performed. In the fixed-bed experiments, 1.0 $\underline{\mathrm{N}}$ sulfuric acid was used as a regenerant. An excess of acid was typically used to ensure complete conversion and, after conversion, deionized distilled water was fed to the column to remove any excess from the column interstitial volume. Therefore, the entire displacement cycle consisted of four steps: sample loading, displacement, regeneration, and rinse.

The principle of displacement chromatography can be applied to CAC, as shown in Fig. 4.2. In this case, the annular region must be provided with four different solutions: (1) the feed to be separated, (2) the displacer, (3) the regenerator, and (4) the rinse. As in conventional displacement chromatography where the displacer is continuously fed following the feed, continuous annular displacement chromatography (CADC) requires the displacer region to be immediately beside the feed region. The displacer region must be long enough (in the $\theta$ direction) to completely displace all of the feed components. Following the displacer region, a regeneration region is necessary to regenerate the sorbent material back to the proper form for loading. Between the regeneration and feed regions tive bed is flushed with an eluent rinse to remove excess regenerant. By continuously pumping feed, displacer, regenerator, and rinse solution into the rotating chromatograph, the CAC can, in principle, be used to perform displacement chromatography in a truly continuous manner.

The CAC used in these experiments was a shortened version of the apparatus used for the metal-ion separations described in Sect. 3.2. It is constructed of 
ORNL DWG 88-119

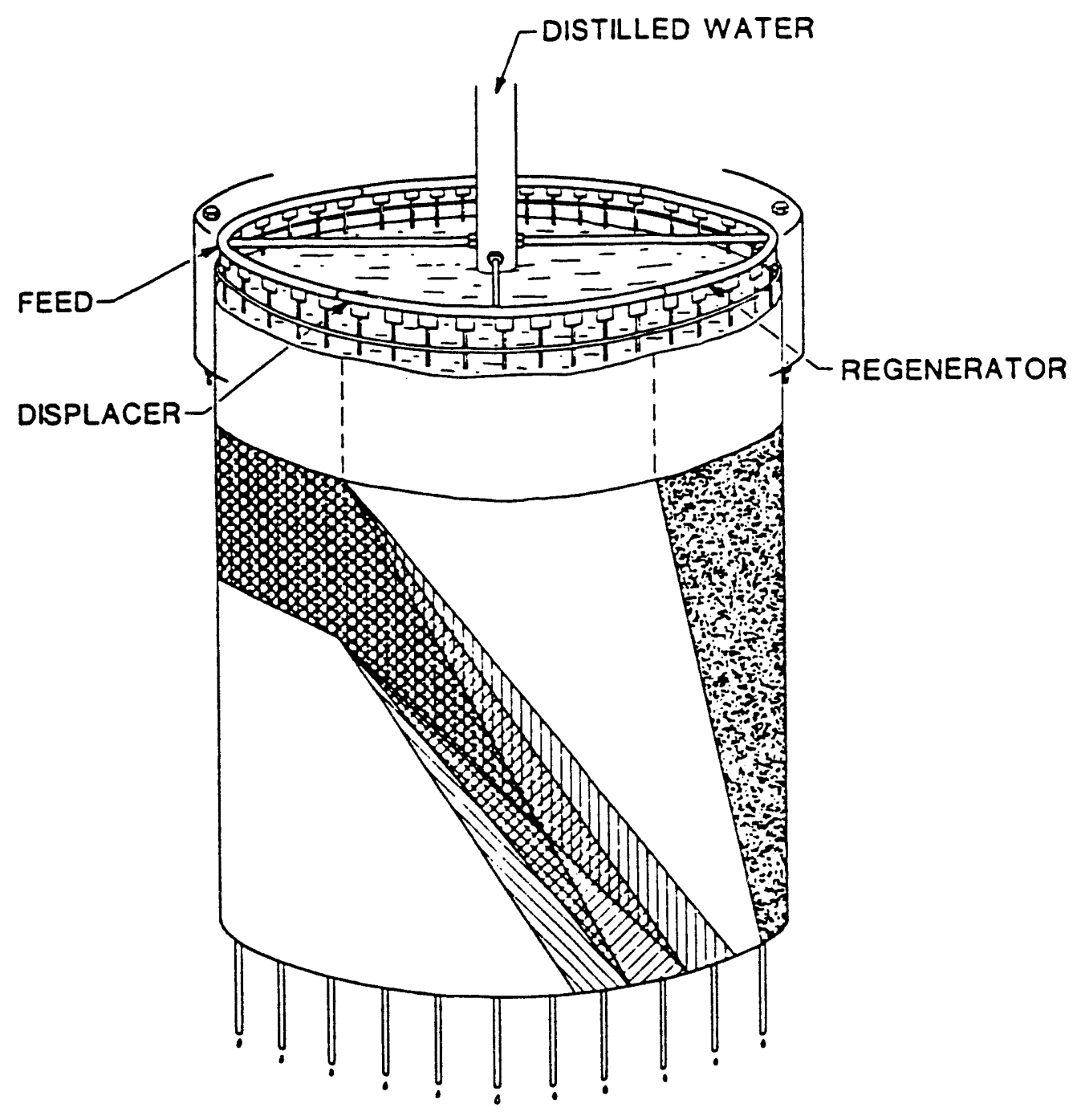

Fig. 4.2. Simplified diagram of a $\mathrm{CAC}$ operating in the displacement mode. 
plexiglass, is $30-\mathrm{cm}$ high, has a $27.9-\mathrm{cm}-\mathrm{OD}$, and has an annulus width of 12.7 $\mathrm{mm}$. The CAC was packed to a height of $22.4 \mathrm{~cm}$ with Dowex resin in the hydrogen form.

The apparatus was then slowly pressurized using a deionized distilled water eluent. Since the head space of the apparatus is closed, increasing the flow rates of the eluent raised the pressure. Pressurization facilitated bed settling and made it easier to pack the upper glass bead layer without disturbing the bed. A layer of glass beads $0.45 \mathrm{~mm}$ in diameter was packed on top of the resin. This layer was 10-cm-deep and prevented convective mixing of the various eluents used in these experiments. As previously mentioned, four different regions are required for continuous annular displacement to occur. Solution from each region must be pumped into a sector of the bed without mixing with other solutions. To accomplish this task a system of four headers, each covering a $90^{\circ}$ section were used. Each header, in turn, contained 1/16-in.-OD stainless steel nozzles spaced $7.5^{\circ}$ apart. Each nozzle could be removed and a plug used in its place so that the sector which each solution covered could be varied. For the displacer and regenerator regions, as long as the sector is long enough to completely displace the feed components and regenerate the bed their sector length did not matter. While the number of degrees a sector could be fed with an eluent could be changed, the basic principle remains the same.

Approximately equal flow through these nozzles was established by the backpressure in each nozzle. To ensure even pressure drop, the pressure drop across each nozzle was measured at constant flow rate and the most uniform group used. By lowering these nozzles into the glass bead layer, even spreading of feed, displacer, and regenerator was obtained without significant mixing. The rinse liquid, deionized distilled water, was fed directly to the head space of the CAC. In this way all of the area not fed by the nozzle distribution system was fed with water. Flow visualization experiments in which blue dextran 2000 was used as a tracer revealed an even flow distribution obtained with this system over the range of experimental conditions reported here. 
A schematic of an unwrapped chromatograph illustrating the various regions is shown in Fig. 4.3. Note that the starting angle, $0^{\circ}$, was chosen as the beginning of the displacer arc. For data collection at the bottom of the column, a peristaltic pump was used to continuously withdraw sample. Readings of $\mathrm{pH}$ were obtained with an Orion Model 701A pH meter and UV absorbance at $200 \mathrm{~nm}$ with a Perkin-Elmer Model 200 UV/Vis Spectrophotometer. The millivolt signals from these instruments were converted to digital signals by an $\mathrm{A} / \mathrm{D}$ conversion board (Data Translation Model 2801) and were continuously recorded using an IBM PC-AT. A schematic of the experimental setup is illustrated in Fig. 4.4. Milroyal Model DC-1-175R pumps were used to feed the displacer, the regenerator, the rinse, and the amino acid feed solution to the column. Since all of the amino acids absorb at $200 \mathrm{~nm}$, concentration profiles could not be determined in this way. However, this method does allow quantification of the amino acid breakthrough angle. Concentration profiles were instead determined from HPLC analyses of samples collected with a mechanical fraction collector. The sample arc depended on the individual run, but typically a sample arc of $2^{\circ}$ was used.

A photograph of the CAC unit operated in displacement chromatography mode with an amino acid feed consisting of glutamic acid, valine, and leucine and using a $0.1 \mathrm{~N} \mathrm{NaOH}$ solution as the displacer is shown in Fig. 4.5. In this photograph, the rotation is counterclockwise. While the amino acids are not colored, the resin assumes a different shade of yellow depending on its ionic form and the solution $\mathrm{pH}$. Thus, the amino acid band is thus visible in the lightly shaded portion of the bed. The darker region is resin in the $\mathrm{Na}^{+}$form, at high $\mathrm{pH}$, and completely free of amino acid. The interface between the light and the dark region is the displacement front. The sharpness of this front reveals the adequacy of the flow distribution system and the lack of significant dispersion in the bed. Finally, to the left of the amino acid band, a region with an intermediate shade of grey is visible. This is resin in the hydrogen form that has been regenerated and rinsed in the two sectors hidden in this photograph to the right of the $\mathrm{NaOH}$ band. 
ORNL DWG 88-118

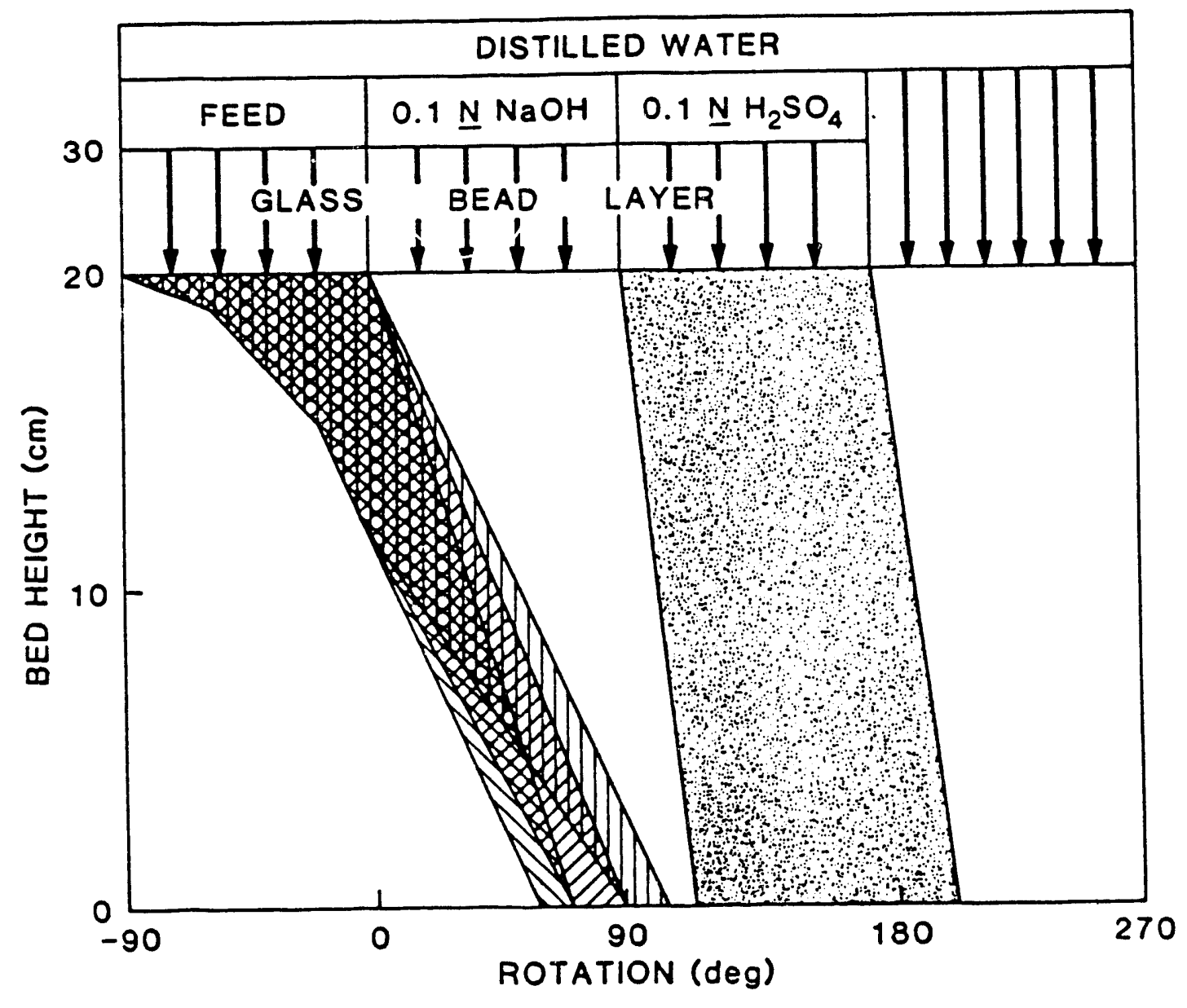

Fig. 4.3. Schematic of eluent distribution within the CAC operating in displacement mode. 


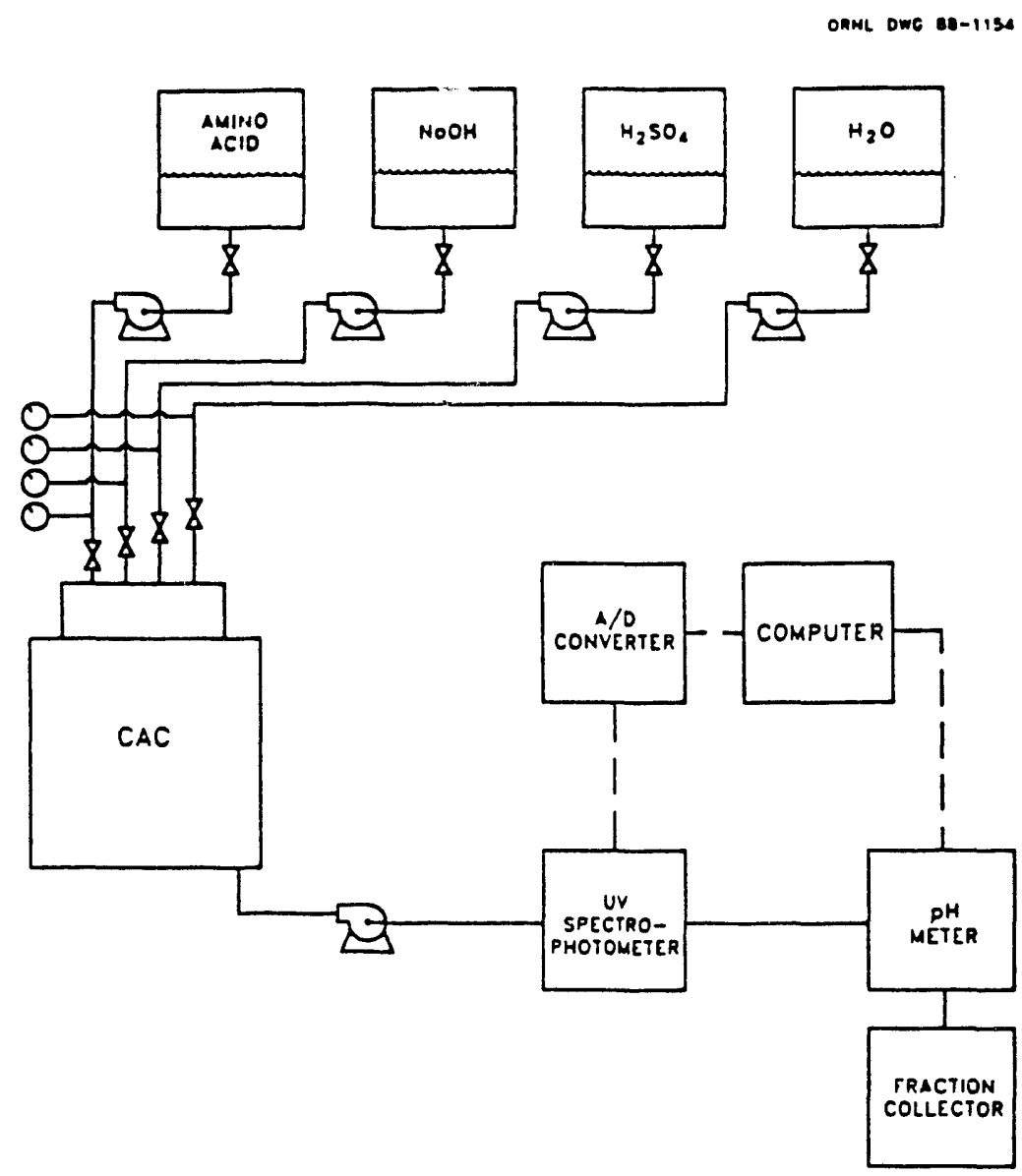

SYSTEM SCHEMATIC

Fig. 4.4. Schematic of the equipment setup used in CAC displacement experiments. 


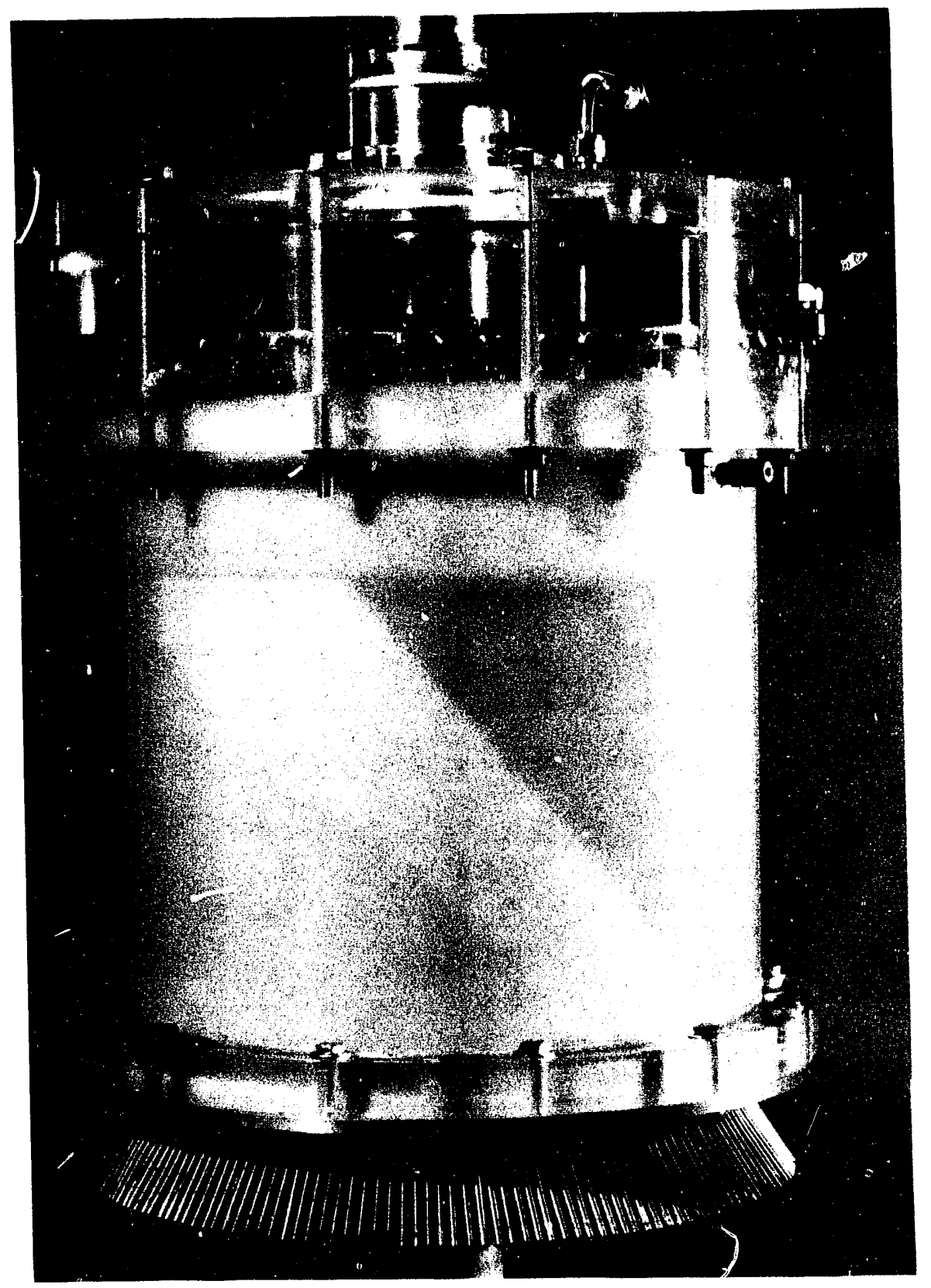

Fig. 4.5. Photograph of the 30.5-cm OD CAC operating in the displacement mode. The band in the middle is amino acid. 


\subsection{THEORETICAL DISCUSSION}

Understanding and modeling the ion exchange of amino acids in a packed bed requires a knowledge of both the solution equilibrium and resin uptake equilibrium. These will be described with the ultimate goal of incorporating them into a model capable of predicting the performance of fixed-bed and CAC operations.

\subsubsection{Solution Equilibrium}

The basic structure of an amino acid is $\mathrm{NH}_{2} \mathrm{CH} \mathrm{RCOOH}$, where $\mathrm{R}$ represents different side chains that make each amino acid unique. These $R$ groups can be either nonpolar (hydrophobic), polar (neutral), acidic, or basic. The latter two groups may be charged depending on the solution $\mathrm{pH}$. For example, $R$ groups for glutamic acid, valine, and leucine (the amino acids used in this work) are $-\left(\mathrm{CH}_{2}\right)_{2} \mathrm{COOH},-\mathrm{CH}\left(\mathrm{CH}_{3}\right)_{2}$ and $-\mathrm{CH}_{2} \mathrm{CH}\left(\mathrm{CH}_{3}\right)_{2}$, respectively. Polar and nonpolar amino acids, such as valine and leucine, exhibit the following equilibria in solution:

$$
\mathrm{NH}_{3}^{+} \mathrm{CHRCOOH} \rightleftharpoons \mathrm{NH}_{3}^{+} \mathrm{CHRCOO}^{-}+\mathrm{H}^{+}, \quad \mathrm{K}_{1},
$$

and

$$
\mathrm{NH}_{3}^{+} \mathrm{CHRCOO}^{-} \rightleftharpoons \mathrm{NH}_{2} \mathrm{CHRCOO}^{-}+\mathrm{H}^{+}, \quad \mathrm{K}_{2},
$$

while acidic amino acids such as glutamic acid have an additional ionizable $R$ group and exhibit

$$
\mathrm{NH}_{2} \mathrm{CHRCOO}^{-} \rightleftharpoons \mathrm{NH}_{2} \mathrm{CHR}^{-} \mathrm{COO}^{-}+\mathrm{H}^{+}, \quad \mathrm{K}_{3}
$$

in addition to Eqs. 4.1 and 4.2. The dissociation constants for reactions 4.1, 4.2 , and 4.3 are $K_{1}, K_{2}$, and $K_{3}$ and they are defined by the mass action law as

$$
K_{1}=\frac{C_{A^{ \pm}} C_{H^{+}}}{C_{A^{+}}}
$$




$$
K_{2}=\frac{C_{A}-C_{H}+}{C_{A^{ \pm}}}
$$

and

$$
K_{3}=\frac{C_{A}=C_{H^{+}}}{C_{A^{-}}}
$$

where $C_{A}+$ is the amino acid cation concentration, $C_{A}$ - is the amino acid anion concentration, $C_{A}=$ is the divalent anion concentration, and $C_{A} \pm$ is the amino acid zwitterion concentration. If the amino acid is dissolved in pure water, the concentrations of the amino acid cation and anion become equal, the predominant form is zwitterionic, and the molecule has no net electrical charge. The $\mathrm{pH}$ at which this occurs is called the isoelectric $\mathrm{pH}$. This is commonly referred to as the isoelectric point and is designated by $\mathrm{pI}$. If the solution $\mathrm{pH}$ is below the amino acid isoelectric point, then the amino acid is positively charged. If the solution $\mathrm{pH}$ is higher than the isoelectric point then the amino acid is negatively charged. The isoelectric $\mathrm{pH}$ can be approximately calculated from the $\mathrm{pK}$ values and the equation

$$
p I=\frac{\left(p K_{1}+p K_{2}\right)}{2} .
$$

The $\mathrm{pK}$ values for glutamic acid, valine, and leucine, and the corresponding calculated $\mathrm{pI}$ values, were given in Table 4.2.

The total "analytical" concentration of amino acid, $C_{A}$, is equal to the sum all of of its ionic forms and can be calculated from

$$
C_{A}=C_{A^{+}}+C_{A^{ \pm}}+C_{A^{-}}+C_{A^{2}}
$$

for the case of glutamic acid. For valine and leucine, which form no divalent anion, the same equation can be used but $C_{A}=$ is set equal to zero. If the total amino acid concentration in a solution and the $\mathrm{pH}$ are known, the amount of each amino acid ion can be calculated. For glutamic acid,

$$
\begin{gathered}
C_{A^{+}}=C_{A} /\left(1+\frac{K_{1}}{C_{H^{+}}}+\frac{K_{1} K_{2}}{C_{H^{+}}^{2}}+\frac{K_{1} K_{2} K_{3}}{C_{H^{+}}^{3}}\right), \\
C_{A^{-}}=C_{A} /\left(1+\frac{C_{H^{+}}}{K_{2}}+\frac{C_{H^{+}}^{2}}{K_{1} K_{2}}+\frac{K_{3}}{C_{H^{+}}}\right),
\end{gathered}
$$


and

$$
C_{A}=C_{A} /\left(1+\frac{C_{H}+}{K_{3}}+\frac{C_{H}^{2}+}{K_{2} K_{3}}+\frac{C_{H}^{3}+}{K_{1} K_{2} K_{3}}\right) .
$$

For valine and leucine, which form no divalent anion, Eqs. 4.9 and 4.10 reduce to

$$
C_{A^{+}}=C_{A} /\left(1+\frac{K_{1}}{C_{H^{+}}}+\frac{K_{1} K_{2}}{C_{H^{+}}^{2}}\right)
$$

and

$$
C_{A^{-}}=C_{A} /\left(1+\frac{C_{H}+}{K_{2}}+\frac{C_{H+}^{2}}{K_{1} K_{2}}\right) .
$$

From these equations and the values of $\mathrm{pK}$ for the various amino acids, the relative amounts of the ionic forms can be calculated as a function of $\mathrm{pH}$. Figure 4.6 is a graphical representation of the amounts of the different ionic forms of the amino acids at equilibrium as a function of $\mathrm{pH}$. In this figure, the maximum amount of the zwitterionic form is at about $\mathrm{pH} 6$ for valine and leucine, and about $\mathrm{pH} 3$ for glutamic acid.

The concentrations of positively and negatively charged species in solution are related by the condition of electroneutrality. For an aqueous solution containing amino acids, $A_{i}$, and $\mathrm{Na}^{+}$and $\mathrm{Cl}^{-}$ions, this condition may be written (Saunders, 1989):

$$
\sum_{i=1}^{N} C_{A_{i}^{+}}+C_{N a i^{+}}+C_{H^{+}}=\sum_{i=1}^{N} C_{A_{i}^{-}}+C_{C 1^{-}}+C_{O H^{-}}+2 \sum_{i=1}^{N} C_{A_{i}} .
$$

The hydroxide concentration is related to the hydrogen ion concentration by the equilibrium

$$
\mathrm{H}_{2} \mathrm{O} \rightleftharpoons \mathrm{H}^{+}+\mathrm{OH}^{-}, \quad K_{W}
$$

with

$$
K_{W}=C_{H^{+}} \cdot C_{H^{-}} \cdot
$$

If Eqs. 4.9, 4.10, 4.11, and 4.16 are used in Eq. 4.14 in place of $C_{A_{1}^{+}}, C_{A_{1}^{-}}$, $C_{A_{1}}$, and $C_{O H_{1}^{-}}$, one obtains a single relationship from which the solution $\mathrm{pH}$ can be calculated given the values of the total amino acid concentrations $C_{A_{1}}$, 


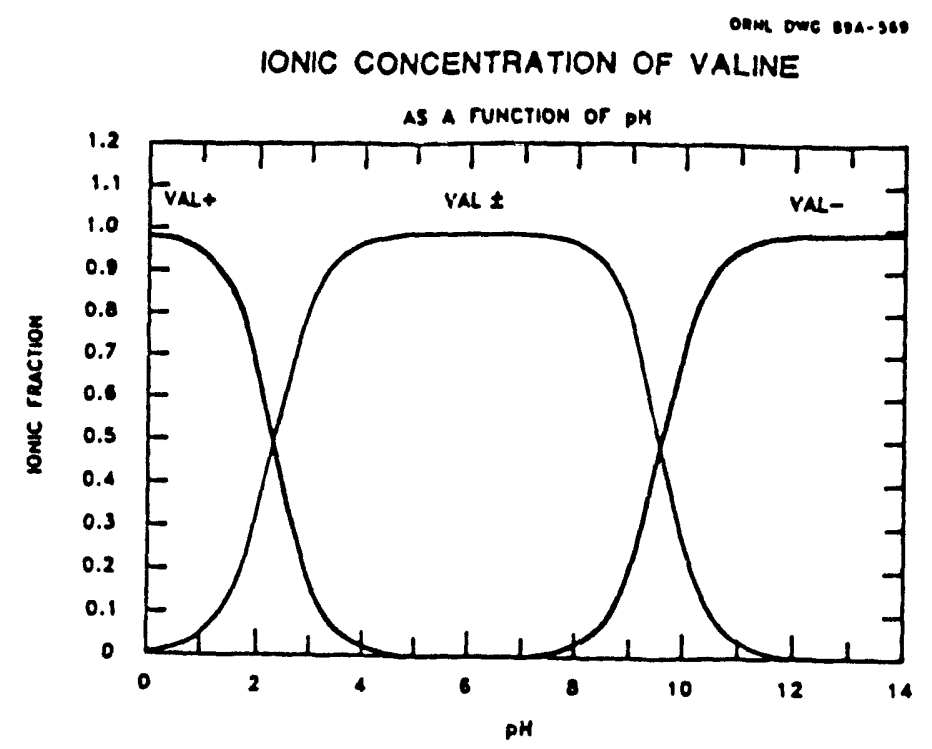

Fig. 4.6(a). Glutamic acid ionic fraction as a function of $\mathrm{pH}$.

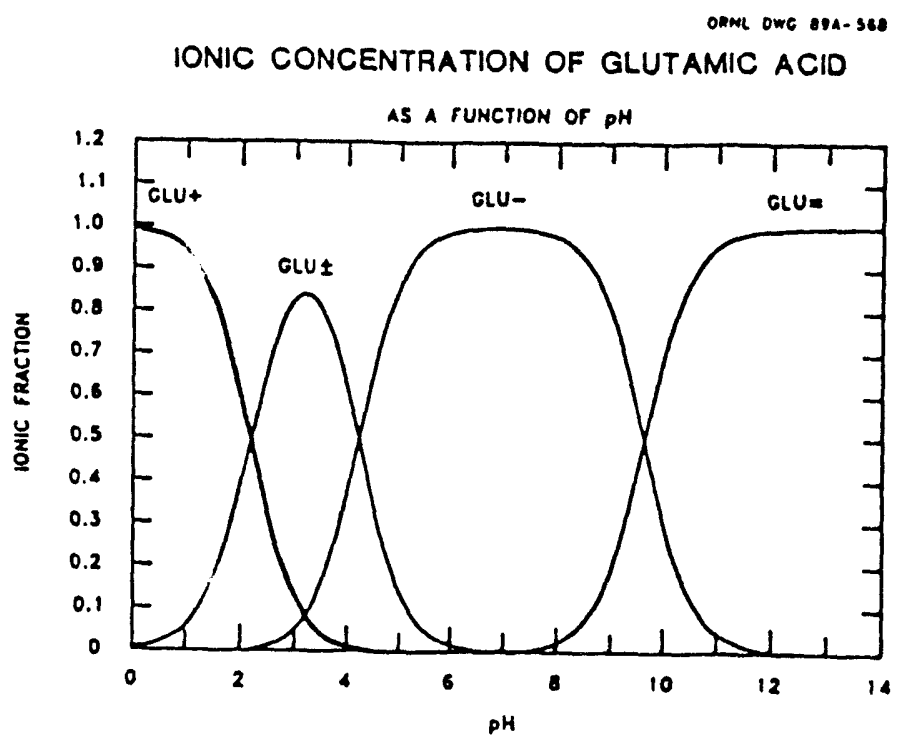

Fig. 4.6(b). Valine ionic fraction as a function of $\mathrm{pH}$. 


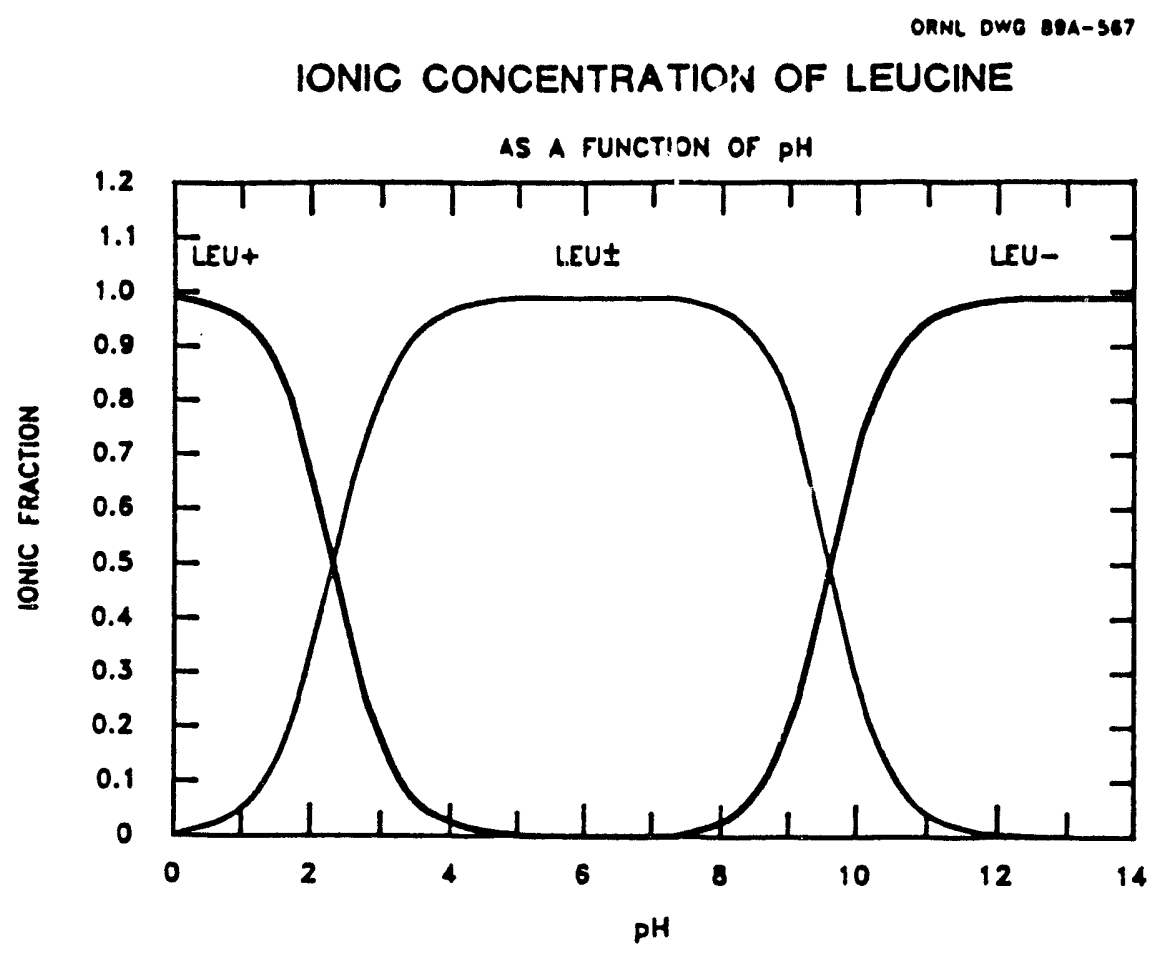

Fig. 4.6(c). Leucine ionic fraction as a function of $\mathrm{pH}$. 
the sodium ion concentration, $C_{N^{+}}$, and the chloride ion concentration, $C_{C_{1-}}$. These concentration values are analytically accessible and permit the calculation of $\mathrm{pH}$ during the ion-exchange process.

In general, a numerical algorithm is required for the solution of Eq. 4.14 for $C_{H}+$. However, as pointed out be Saunders (1989), however, exact solutions are possible away from the isoelectric $\mathrm{pH}$ for solutions containing a single amino acid. If $C_{\mathrm{Na}^{+}}=C_{\mathrm{Cl}^{-}}$and a single amino acid is present, solution of Eq. 4.14 provides a $\mathrm{pH}$ equal to the isoelectric $\mathrm{pH}$ of the amino acid.

\subsubsection{Uptake Equilibrium: Results and Modeling}

The experimental equilibrium uptake measurements for glutamic acid, valine, and leucine obtained with the technique described in Sect. 4.3.2 are given in Fig. 4.7 (Dye, 1988). The total amino acid uptake (in $\mathrm{mmol} / \mathrm{g}$ dry resin in hydrogen form) is $q_{A}$ and $C_{A}$ is the total amino acid or analytical amino acid concentration in solution. As pointed out in Sect. 4.3.2, these experiments were carried out at constant coion concentration, not at constant $\mathrm{pH}$. Thus, the $\mathrm{pH}$ varies from point to point on each curve. As discussed by Dye (1988), the equilibrium uptake approaches a maximum as the coion concentration goes to zero. For these conditions, the $\mathrm{pH}$ approaches the isoelectric $\mathrm{pH}$ and competition by hydrogen ions is minimized. As the $\mathrm{Cl}^{-}$concentration is increased, the $\mathrm{pH}$ is reduced and the equilibrium uptake of amino acid is decreased by the stronger competition of hydrogen ions for the resin. Ion-exchange equilibrium for an amino acid cation with a cation-exchange resin in the hydrogen form may be described by the following equation:

$$
\mathrm{R}^{\prime} \mathrm{H}+\mathrm{NH}_{3}^{+} \mathrm{CHRCOOH} \rightleftharpoons \mathrm{R}^{\prime} \mathrm{NH}_{2} \mathrm{CHRCOOH}+\mathrm{H}^{+},
$$

where $R^{\prime}$ represents the negatively charged functional groups of the resin. This equation treats the uptake process as one involving only ion exchange in stoichiometric proportion. The assumption is justifiable at high solution concentrations 


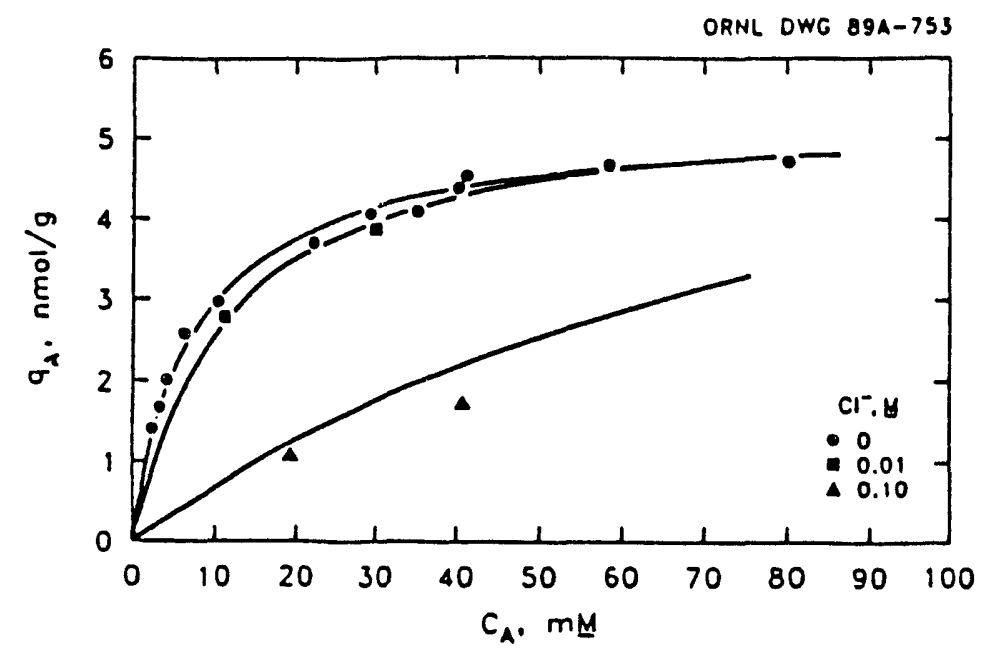

Fig. 4.7(a). Uptake of glutamic acid ions by cation-exchange resin Dowex 50W-X8. Counterion: $\mathrm{H}^{+}$; Coion: $\mathrm{Cl}^{-}$.

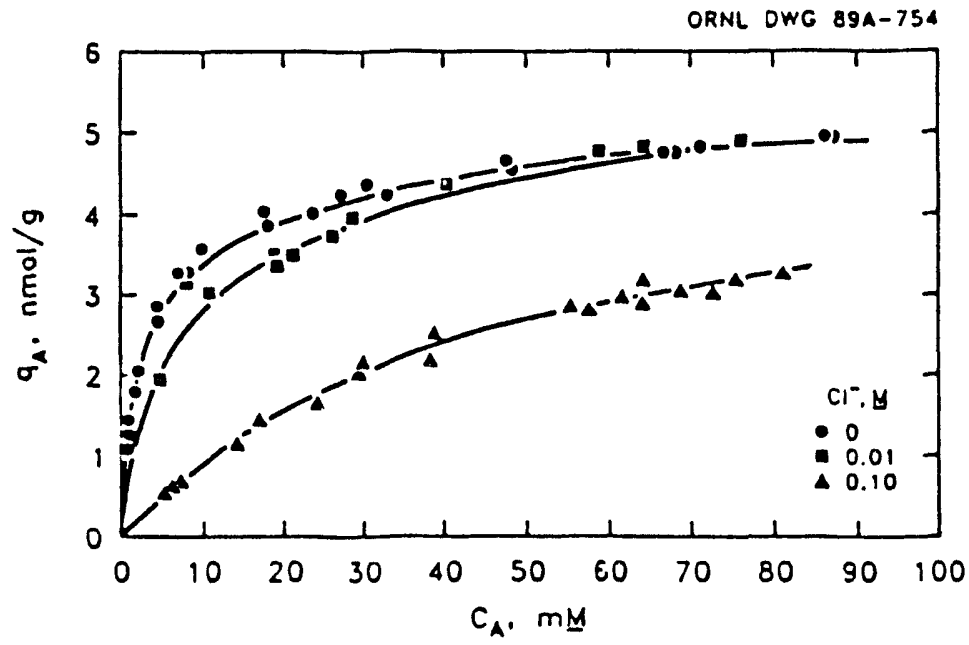

Fig. 4.7(b). Uptake of valine ions by cation-exchange resin Dowex 50W-X8. Counterion: $\mathrm{H}^{+}$; Coion: $\mathrm{Cl}^{-}$. 
ORNL DWG 89A-755

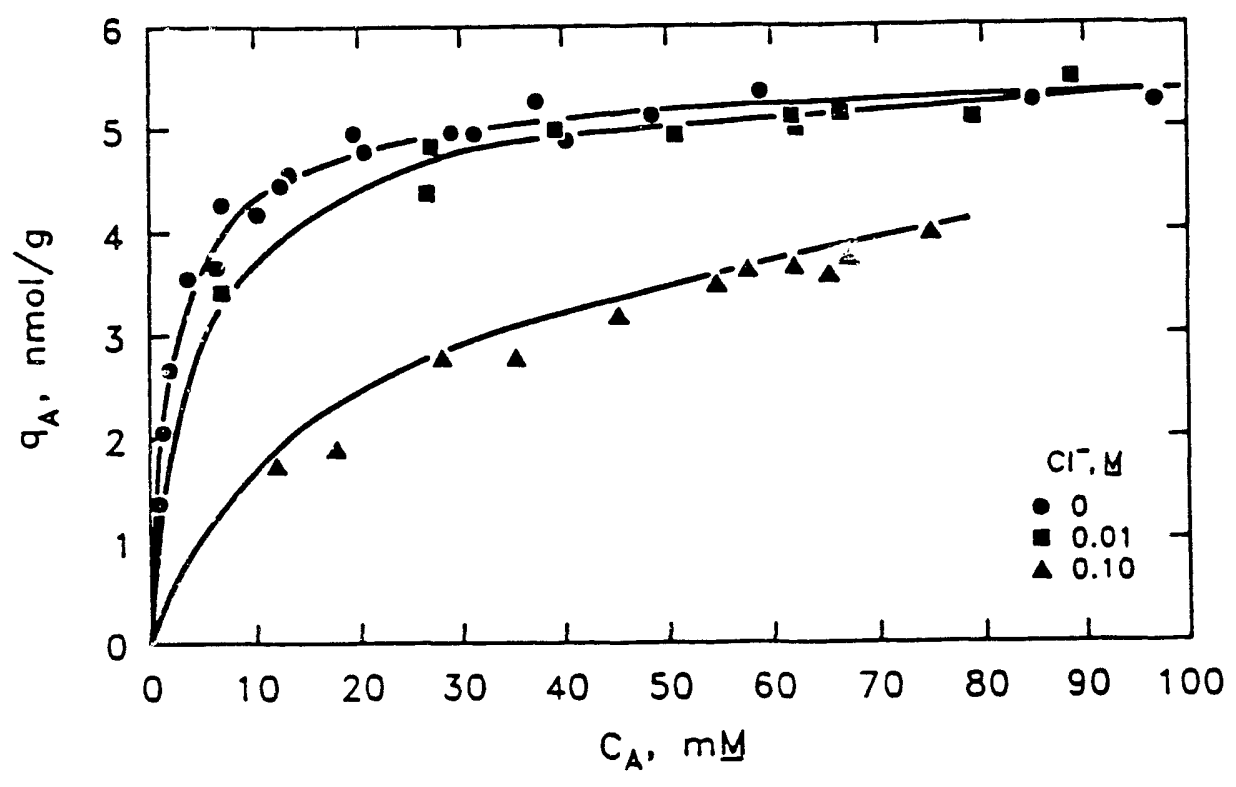

Fig. 4.7(c). Uptake of leucine ions by cation-exchange resin Dowex 50WX8. Counterion: $\mathrm{H}^{+}$; Coion: $\mathrm{Cl}^{-}$. 
with high-capacity resins such as Dowex 50 (Saunders, 1989). For these conditions, which are typical of process applications, nonionic adsorption is negligible. For binary ion exchange of an amino acid cation with a hydrogen ion, an equilibrium constant based on the mass action law can be defined for Eq. 4.17 as

$$
K_{A, H}=\frac{C_{H}+q_{A}}{C_{A}+q_{H}} .
$$

Since $q_{A}+q_{H}=q^{\circ}$, which is the total resin capacity, Eq. 4.18 can be rewritten as

$$
Y_{A}=\frac{K_{A, H} X_{A}}{X_{H}+K_{A, H} X_{A}},
$$

where $X_{A}$ is the ionic fraction of amino acid cations in solution defined by

$$
X_{A+}=\frac{C_{A^{+}}}{C_{A^{+}}+C_{H^{+}}}
$$

and $Y_{A}$ is the fractional uptake of amino acid by the resin defined by

$$
Y_{A}=\frac{q_{A}}{q^{\circ}}
$$

$K_{A, H}$ is an apparent equilibrium "constant" that may, in fact, depend on resin and solution composition.

The binary equilibrium data in Fig. 4.7 are replotted in the form suggested by Eq. 4.19 as $X-Y$ plots in Fig. 4.8 (Dye, 1988). Notice that the data obtained at different $\mathrm{pH}$ levels fall on a single line. This indicates that the uptake of amino acid depends solely on the ionic fraction of amino acid cations and that nonionic adsorption is relatively unimportant. For the general case with $n$ amino acids, the liquid ionic fraction of ion $i$ is defined as

$$
X_{i}=\frac{C_{A_{1}^{+}}}{\sum_{\ell=1}^{n} C_{A_{1}^{+}}},
$$

and the fractional uptake for a resin with a fixed number of binding sites is defined as

$$
Y_{A_{i}}=\frac{q_{A_{1}}}{q^{\circ}}=\frac{K_{i} X_{A_{1}}}{\sum_{\ell=1}^{n} K_{\ell} X_{A_{\ell}}+X_{M}}
$$




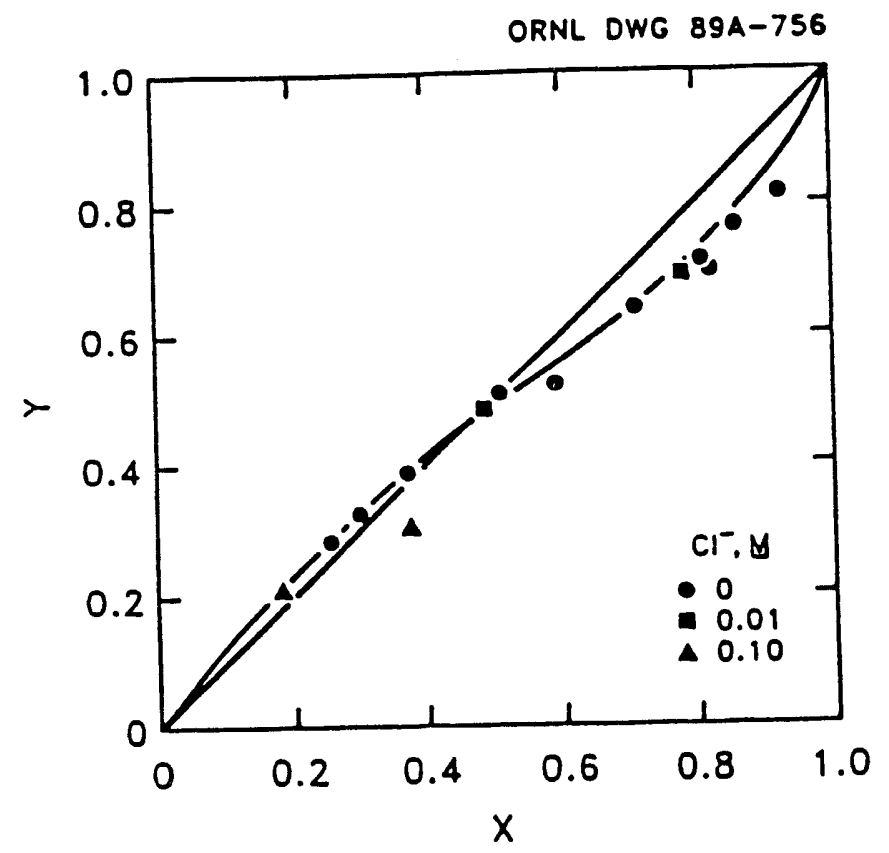

Fig. 4.8(a). Ion-exchange equilibrium for binary ion exchange of glutamic acid cations and hydrogen ions on Dowex 50W-X8.

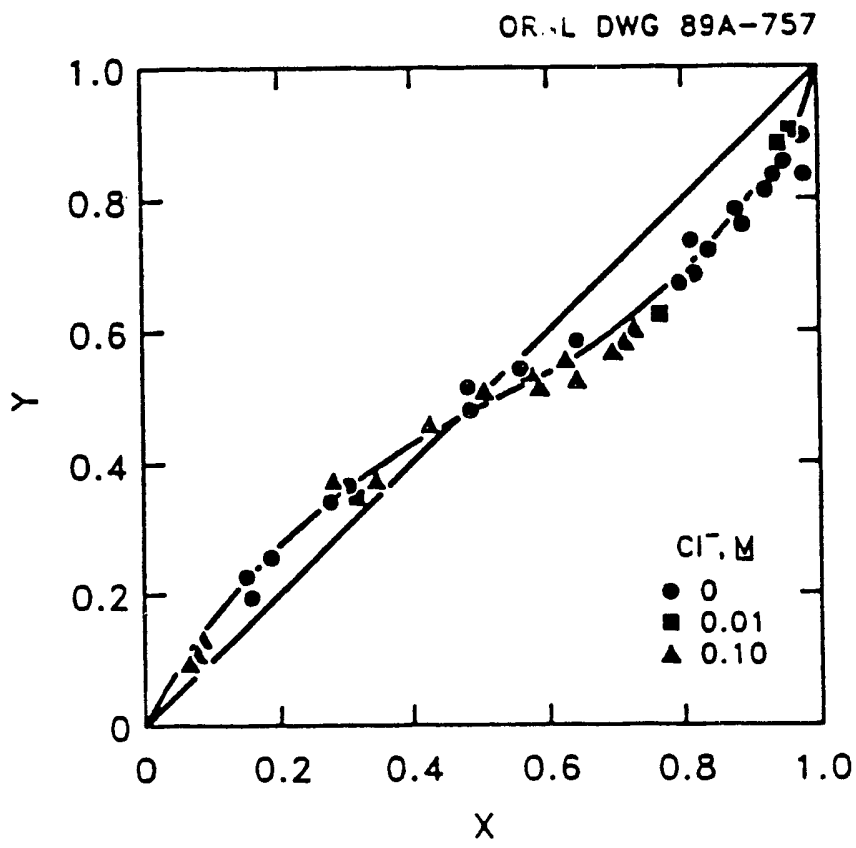

Fig. 4.8(b). Ion-exchange equilibrium for binary ion exchange of valine cations and hydrogen ions on Dowex 50W-X8. 


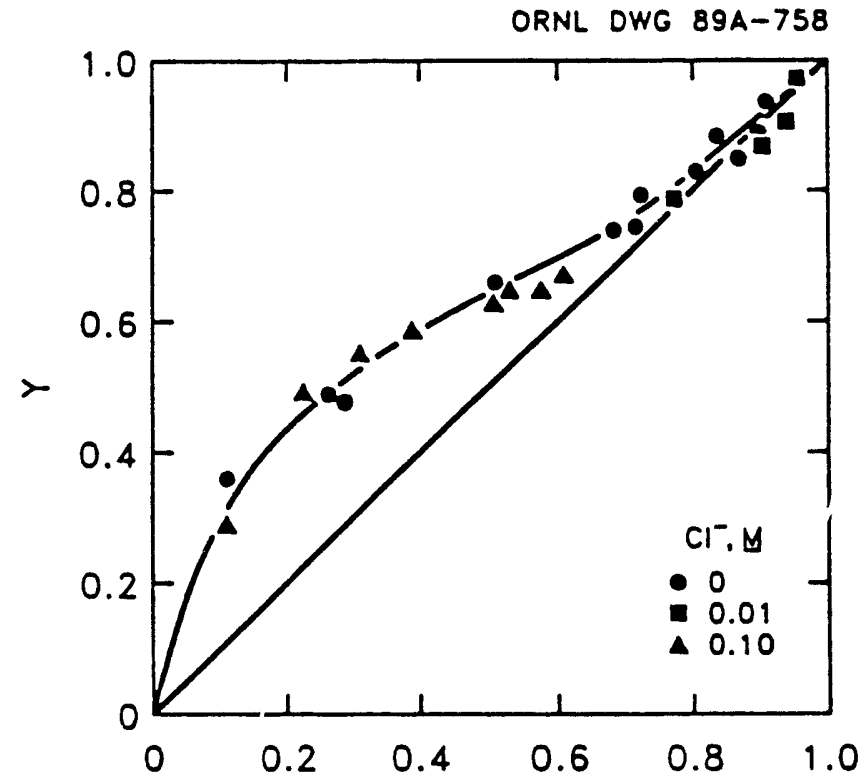

Fig. 4.8(c). Ion-exchange equilibrhum for binary ion exchange of leucine cations and hydrogen ions on Dowex 50W-X8. 
The separation factor, $S_{i, j}$, between ions $i$ and $j$ can be thought of as the ratio of distribution coefficients and is defined as

$$
S_{i, j}=\frac{\frac{Y_{i}}{X_{i}}}{\frac{Y_{j}}{X_{j}}}=\frac{Y_{i} X_{j}}{X_{i} Y_{j}}=\frac{K_{i}}{K_{j}}
$$

If all the $K_{i} \mathrm{~s}$ in Eq. 4.23 are constant, then $S_{i, j}$ is constant. If $K_{i}=K_{j}$, the separation factor, $S_{i, j}$, equals 1 and the solid and liquid phase concentrations are equal. If $S_{i, j}$ is greater than 1 the solid phase concentration is higher than the liquid phase concentration and the isotherm is favorable. If $S_{i, j}$ is less than 1 the liquid phase concentration is higher than the solid phase concentration and the isotherm is unfavorable. Ionic-exchange equilibrium data in Fig. 4.8 illustrate this point. Here, the diagonal corresponds to a separation factor of 1 and divides the graph into two regions, favorable and unfavorable. Points above the line exhibit favorable equilibrium while those below the line exhibit unfavorable equilibrium. In the systems studied here, L-leucine is favorable, and this can be easily seen from the figure. L-valine and L-glutamic acid, however, exhibit composite behavior and are favorable at low concentrations and unfavorable at high concentrations.

The behavior of amino acids in solution and the mechanism of ion exchange have been covered in this and the previous section. It is desired to be able to quantitatively predict amino acid uptake of the adsorbent from a known liquid phase composition. It is also desired that the model will not only predict pure component but also multicomponent, isotherms. Saunders (1989) has had great success in modeling nonconstant separation factor ion-exchange isotherms with a model adapted from Novosad and Myers (1982) and Myers and Byington (1986).

The model assumes that the ion exchanger contains "high energy" and "low energy" ion-exchange sites. These high energy sites are filled first which is why the slope of the uptake curves is large at low concentrations. Only after the high energy sites are filled are the low energy sites filled causing the slope of the uptake curve to decrease at high concentrations. The model assumes that there 
are $n+1$ of these sites with a binomial distribution of energy. The probability that a site is of type $\ell$ is defined by

$$
p_{\ell}=\left(\begin{array}{l}
n \\
\ell
\end{array}\right) p^{\ell}(1-p)^{n-\ell}, \quad 0<p<1,
$$

where $p$ is the skewness of the energy distribution function. The energy of adsorption for ion $j$ on site $\ell$ is

$$
E_{\ell, j}=\bar{E}_{j}+\frac{\ell-n p}{\sqrt{n p(1-p)}} \sigma_{j}
$$

where $\bar{E}_{j}$ and $\sigma_{j}$ are the average and standard deviations of the adsorption energy distribution. If the adsorption of ion $j$ on site $\ell$ can be described by

$$
\frac{q_{\ell, j}}{q_{\ell \circ}}=\frac{C_{\ell, j} X_{j}}{\sum_{j=1}^{N} C_{\ell, j} X_{j}}
$$

where $q_{\ell}$ is the total number of $\ell$ type sites, and $N$ is the total number of different ions that can be exchanged (the counterions), then $C_{\ell, j}$ is

$$
C_{\ell, j}=\bar{C}_{j} \exp \left(\frac{E_{\ell, j}-\bar{E}_{j}}{R T}\right) \text {. }
$$

By summing over the $n+1$ sites, the total uptake of ion $j$ may be found from

$$
\frac{q_{j}}{q_{\max }}=\sum_{\ell=0}^{n}\left[\frac{C_{\ell, j} p_{\ell} X_{j}}{\sum_{j=1}^{N} C_{\ell, j} X_{j}}\right]
$$

The separation factor, $S_{i, j}$, for ion $i$ relative to ion $j$ for binary ion exchange becomes

$$
S_{i, j}=\bar{S}_{i, j} \frac{\bar{S}_{i, j} W^{U+V} X_{i}+\left[W^{U}(1-p)+W^{V} p\right] X_{j}}{\bar{S}_{i, j}\left[W^{V}(1-p)+W^{U} p\right] X_{i}+X_{j}}
$$

where

$$
\begin{aligned}
& \bar{S}_{i, j}=\exp \left(\frac{\bar{E}_{i}-\bar{E}_{j}}{R T}\right) \\
& W_{i, j}=\exp \left(\frac{\sigma_{i}-\sigma_{j}}{R T}\right)
\end{aligned}
$$




$$
U=\frac{-p}{\sqrt{p(1-p)}}
$$

and

$$
V=\frac{1-p}{\sqrt{p(1-p)}}
$$

Once all of the $\bar{S}_{i, j}$ values are determined, the uptake of ion $i$ can be calculated from

$$
Y_{i}=\frac{q_{i}}{q_{\max }}=\frac{X_{i} S_{i, j}}{\sum_{k=1}^{N} X_{k} S_{k, j}} .
$$

This is an empirical model that assumes from the very start that it is impossible to predict a priori ion-exchange equilibrium. Therefore, for binary ion exchange, this model, like others, requires the experimental determination of the equilibrium isotherm. The approach was to develop a model that was thermodynamically sound, used as few parameters as possible, and minimized the number of experiments. Myers and Byington (1986) showed that Eqs. 4.30 to 4.35 satisfy the Gibbs-Duhem relation. This is a three-parameter model and the parameters to be evaluated are the (1) average energy of the adsorption energy distribution, $\bar{E}_{i} ;(2)$ standard deviation, $\sigma$; and (3) skewness of the distribution of the types of energy sites, $p$. In practice, these values cannot be directly measured, from the numerical values of the parameters $S_{i, j}$ and $W_{i, j}$ determined from a fit of the data. These parameters were fitted by minimizing the sum of the residuals and are presented in Table 4.3 (Dye, 1988). The results of calculations based on this model are the solid lines shown in Figs. 4.7 and 4.8. It is easy to see that there is good agreement between the model calculations and the experiments.

This model was extended by Saunders (1989) for use with multicomponent equilibrium. The separation factor for ion $i$ with reference ion $j$, becomes

$$
S_{i, j}=\frac{Y_{i} X_{j}}{Y_{j} X_{i}}=\bar{S}_{i, j} \frac{\sum_{k=1}^{N}\left\{\bar{S}_{k, j} X_{k} W_{k, j}^{U+V}\left[(1-p) W_{i, k}^{U}+p W_{i, k}^{V}\right]\right\}}{\sum_{k=1}^{N}\left\{\bar{S}_{k, j} X_{k} W_{k, j}^{U+V}\left[(1-p) W_{j, k}^{U}+p W_{j, k}^{V}\right]\right\}} .
$$

The fitting parameters determined from the binary experiments are used here. Table 4.4 (Dye, 1988) presents some experimental and calculated multicompo- 
Table 4.3 Ion Exchange Equilibrium Parameters ${ }^{\dagger}$

\begin{tabular}{llll}
\hline & $\bar{S}$ & $W$ & $p$ \\
\hline L-GLU & 1.0 & 0.35 & 0.50 \\
L-VAL & 1.0 & 0.25 & 0.50 \\
L-LEU & 5.0 & 3.0 & 0.50 \\
\hline
\end{tabular}

${ }^{\dagger}$ Dye (1988) 
nent equilibrium using the values of $\bar{S}_{i, j}, W_{i, j}$, and $p$ in Table 4.3. The prediction is quite good.

Therefore, for prediction of multicomponent equilibrium, this model only requires measurement of binary equilibrium data with respect to a specific counterion. This implies that for a system of five components, A through E, only binary equilibrium data on $A$ through $D$ with counterion $E$ are required. Other models typically require binary, ternary, and quaternary data so that the number of experiments to be performed increases rapidly with the number of components. This becomes impractical for systems with large numbers of components. The power of this model is its ability to predict equilibrium for a system of $N$ components from $N-1$ binary experiments.

A further simplification of this model is the case where $W_{i, j}=1$. In this case, $S_{i, j}=\bar{S}_{i, j}$ and the separation factors are constant. Figures 4.9 and 4.10 compare nonconstant and constant separation factor calculations for the experimental system under investigation here. Since the separation between glutamic acid and valine is due to differences in their isoelectric points, the assumption of constant separation factors for these two components should be a good approximation. The valine-leucine separation, on the other hand, is due to the large differences in their specific affinity for the resin. Since the difference is so large, the assumption of constant $S$ should prove reasonable. Constant separation factors will thus be assumed to simplify mathematical modelling. However, the separation factors used will be the average values of $\bar{S}$ determined from the equilibrium model described above.

\subsubsection{Bed Dynamics}

The equilibrium data and models developed can be incorporated in models to describe bed dynamics with the aim of predicting the concentration histories in a fixed bed and the concentration profiles in the CAC. A one-dimensional, 
(Dye, 1988)

Table 4.4

Multicomponent Ion-Exchange Equilibrium

on Dowex 50W-X8

Leucine/Valine/Glutamic Acid/Hydrogen

$\left[\mathrm{Cl}^{-}\right]=0 \mathrm{M}$

$C$ in $\mathrm{mM}, q$ in $\mathrm{mmol} / \mathrm{g}$ 


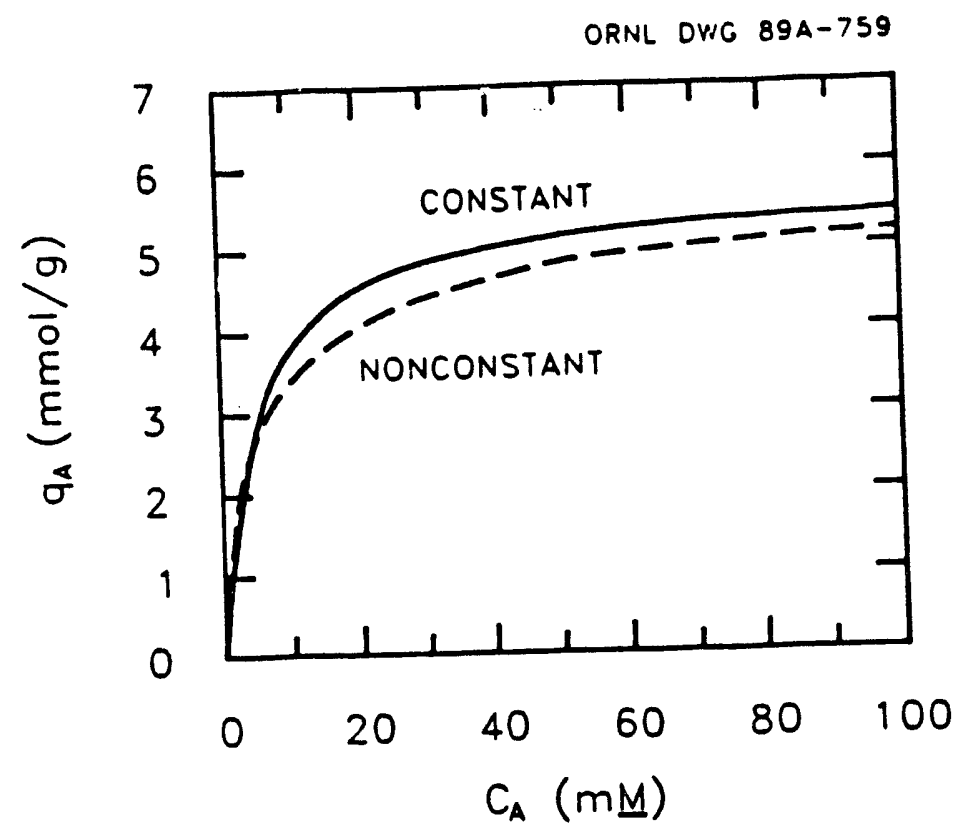

Fig. 4.9(a). Comparison of constant and nonconstant separation factor isotherms for glutamic acid uptake equilibrium on Dowex $50 \mathrm{~W}-\mathrm{X} 8$.

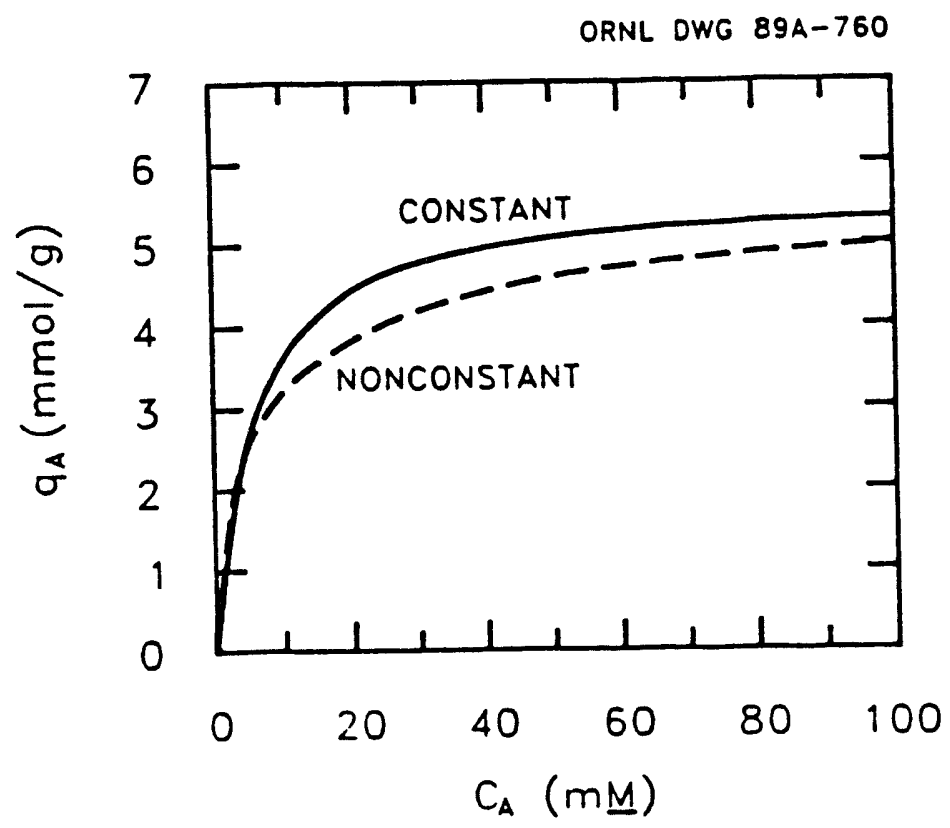

Fig. 4.9(b). Comparison of constant and nonconstant separation factor isotherms for valine uptake equilibrium on Dowex $50 \mathrm{~W}-\mathrm{X} 8$. 


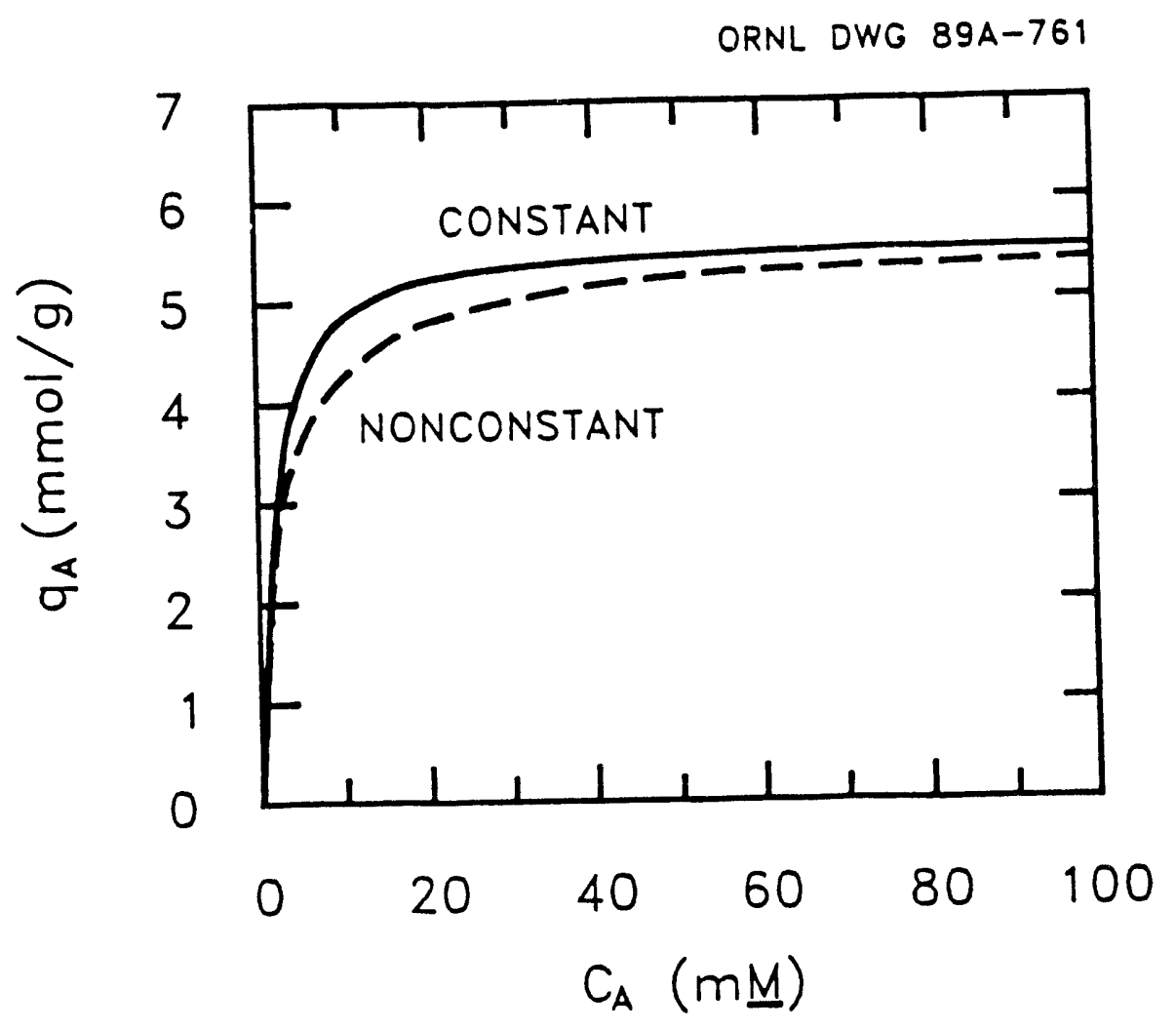

Fig. 4.9(c). Comparison of constant and nonconstant separation factor isotherms for leucine uptake equilibrium on Dowex 50W-X8. 


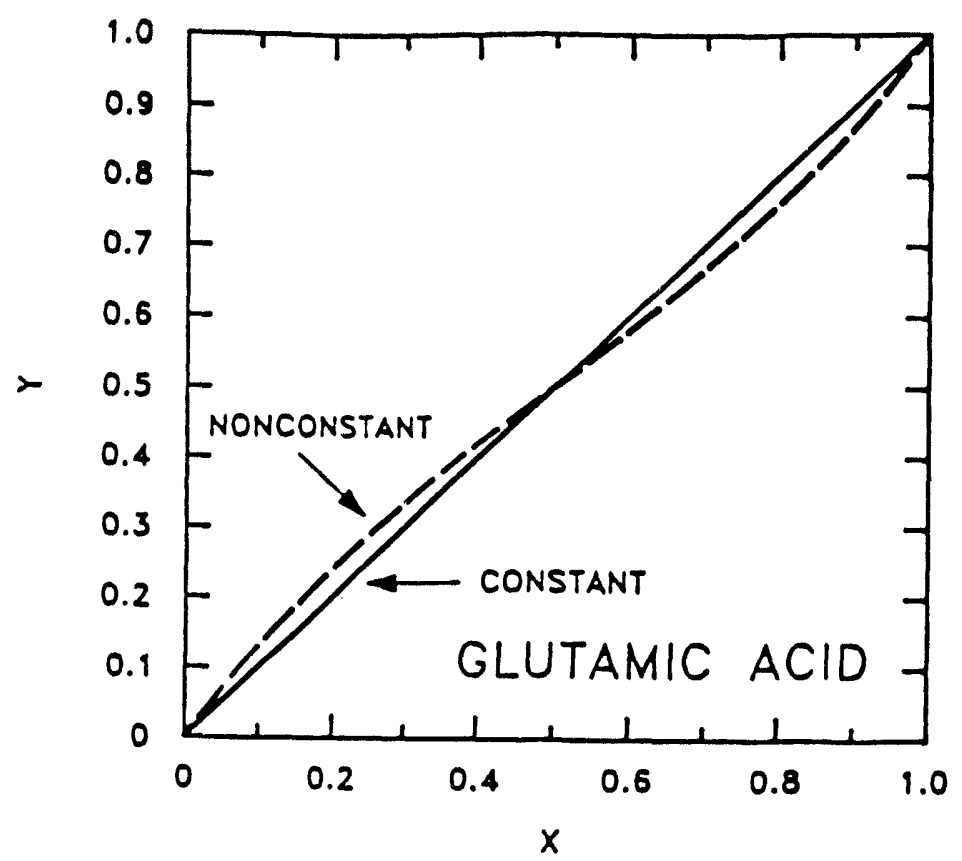

Fig. 4.10(a). Comparison of constant and nonconstant separation factors for binary ion-exchange equilibrium for glutamic acid cations and hydrogen ions on Dowex 50W-X8. ORNL DWG 89A-572

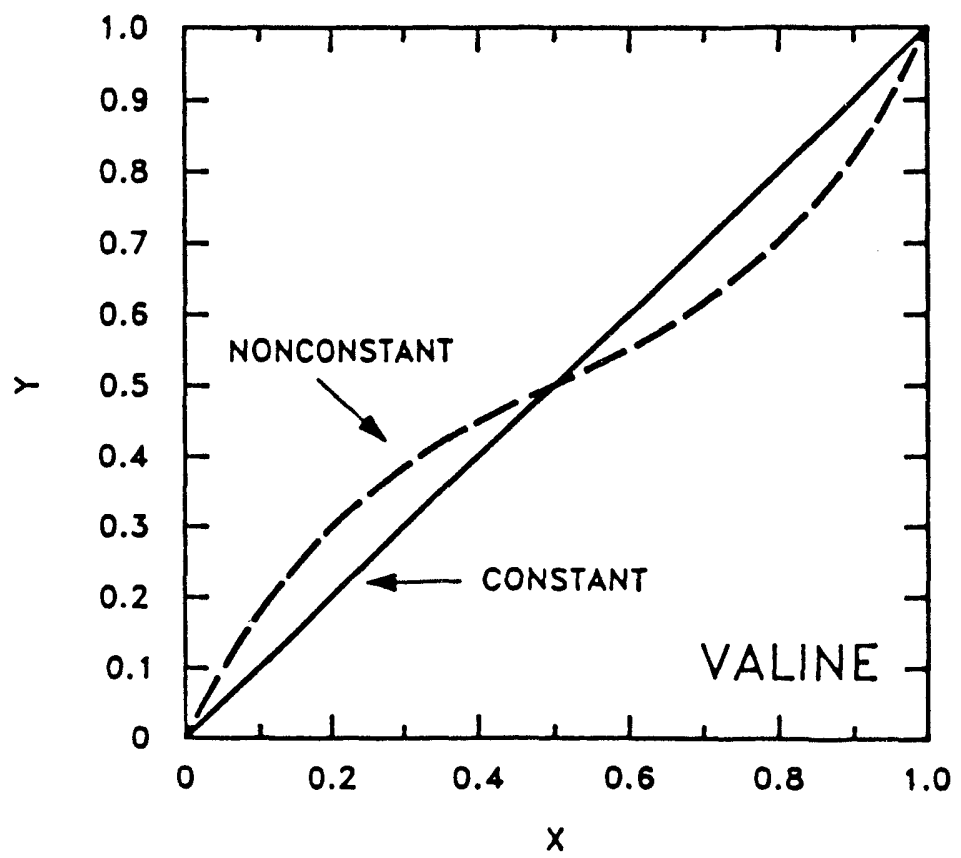

Fig. 4.10(b). Comparison of constant and nonconstant separation factors for binary ion-exchange equilibrium for valine cations and hydrogen ions on Dowex 50W-X8. 
ORNL DWG 89A-570

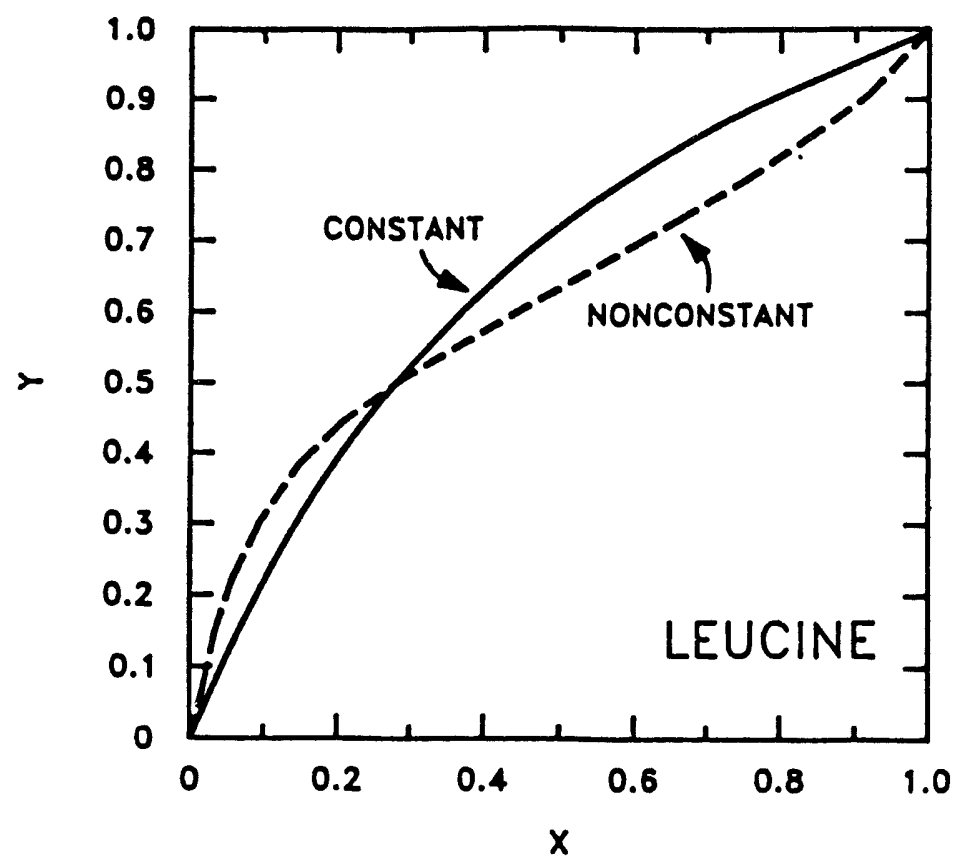

Fig. 4.10(c). Comparison of constant and nonconstant separation factors for binary ion-exchange equilibrium for leucine cations and hydrogen ions on Dowex 50W-X8. 
unsteady-state, material balance on solute $C_{i}$ in a fixed bed may by expressed as

$$
\epsilon \frac{\partial C_{i}}{\partial t}+\rho_{b} \frac{\partial q_{i}}{\partial t}+u \frac{\partial C_{i}}{\partial z}=0
$$

where axial dispersion is neglected. Transport to and from the solute may be described by

$$
\rho_{b} \frac{\partial q_{i}}{\partial t}=k_{o_{i}} a\left(C_{i}-C_{i}^{*}\right),
$$

where $k_{o_{i}} a$ is the overall mass transfer coefficient and $C_{i}^{*}$ is the liquid phase concentration in equilibrium with the solid phase. In general, solutions to these equations can be accomplished analytically for the case of simple adsorption isotherms or numerically for complex isotherms. A third approach is to assume local equilibrium. This implies that transport between the solid and liquid phases is instantaneous and need not be considered. Conditions where the assumption of local equilibrium is best made are for columns packed with small particles where a slow fluid velocity is used. Use of this assumption also extends the complexity of adsorption isotherms that can be solved analytically (Helfferich and Klein, 1970; Ruthven, 1984).

Unfortunately, the amino acids studied here exhibit complex equilibrium so that Eqs. 4.37 and 4.38 cannot be solved analytically. Also, simplification of the isotherm (even to constant separation factors) did not allow analytical solution because of the system dissociation reactions occuring in solution. Thus, two different approaches were taken. In the first one, the Glueckauf method (Glueckauf, 1949) was used to determine the achievable product concentrations when the displacement separation train is fully developed. This method consists of a very simple graphical procedure and is described below. The second approach makes use of a finite difference approximation of Eq. 4.37, which is equivalent to describing the column as a series of discrete equilibrium stages. This approach required a numerical solution of the resulting ordinary differential equations, but allowed a qualitative prediction of the initial development of a concentration band during the displacement process. 


\subsubsection{Glueckauf's Method}

The achievable product concentrations in displacement chromatography may be adequately predicted by Glueckauf's method (Glueckauf, 1949; Ruthven, 1984). The procedure is shown in Fig. 4.11. In this figure, the pure component uptake of glutamic acid, valine, and leucine is shown as a function of the solution concentration at zero coion concentration. A horizontal line is used to describe the essentially irreversible uptake of $\mathrm{NaOH}$ at the resin capacity of 5.6 meq/g dry resin. In displacement chromatography, the displacer concentration velocity, $v_{c}^{N a}$ (see Eq. 1.1), is larger than any of the individual component velocities, $v_{c}^{G L U}, v_{c}^{V A L}$, or $v_{c}^{L E U}$. If the column is long enough, the displacer overtakes the feed components and competes favorably with them for adsorption sites. If competitive equilibrium does not exist between the displacer and the components to be separated displacement cannot be achieved. Eventually, all components attain the same concentration velocity and are said to be "coherent" (Helfferich and Klein, 1970). These constant velocity concentrations are called "isotachic" concentrations and are determined from a solution of the equations

$$
v_{c}^{N a}=v_{c}^{G L U}=v_{c}^{V A L}=v_{c}^{L E U} .
$$

Assuming that the fluid velocity is constant within a column, Eq. 4.37 yields

$$
\frac{\Delta q_{N a}}{\Delta C_{N a}}=\frac{\Delta q_{G L U}}{\Delta C_{G L U}}=\frac{\Delta q_{V A L}}{\Delta C_{V A L}}=\frac{\Delta q_{L E U}}{\Delta C_{L E U}} .
$$

This is shown graphically in Fig. 4.11. Here, a chord was drawn between the initial $\mathrm{NaOH}$ concentration in the bed $(0 \mathrm{mM})$ and the point on the displacer equilibrium curve corresponding to the displacer feed concentration (e.g., 100 $\mathrm{mM}$ ). This chord intersects the amino acid equilibrium curves at their isotachic (or constant velocity) concentrations. For a displacer concentration of $100 \mathrm{mM}$, the isotachic concentrations were approximately $85 \mathrm{mM}$ for glutamic acid and valine, and $96 \mathrm{~m} \underline{\mathrm{M}}$ for leucine. $\mathrm{f}$ the column is long enough and if mass transfer 


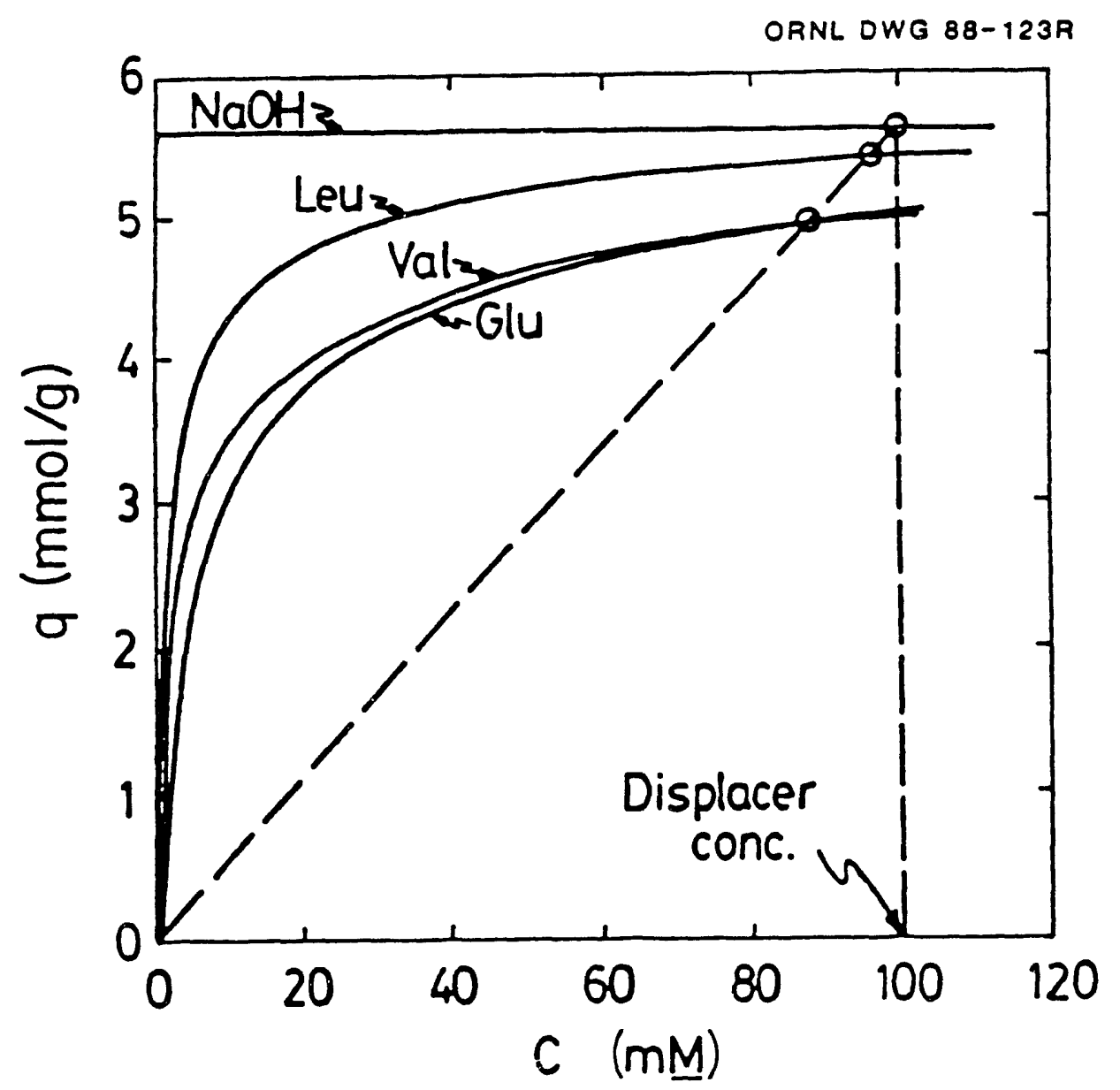

Fig. 4.11. Predicted outlet concentration by method of Glueckauf. 
resistances are small, then each component will reach this concentration. If the displacer concentration is changed, a new chord must be drawn and the new isotachic concentrations determined.

It is interesting to note that although the valine and glutamic acid have essentially the same pure component isotherms, they will still be separated in the dispiacement train as a result of differences in their isoelectric $\mathrm{pH}$ values. For example, at $\mathrm{pH} 5.97$, (which is the $\mathrm{pI}$ of valine), glutamic acid (which has a $\mathrm{pI}$ of 3.22), will be negatively charged and will move further down the column to be reabsorbed downstream of the valine band. The separated components will thus exit from the column in order of increasing $\mathrm{pH}$.

\subsubsection{Equilibrium Stage Model}

The graphical method of Glueckauf is only sufficient to predict the outlet concentrations at isotachic conditions. It is relatively easy to use, and provides valuable information, but it has no application to nonisotachic conditions. A one-dimensional stage model assuming local equilibrium was used here to simulate displacement experiments and accounts for the amphoteric nature of the amino acids. The assumption of equilibrium in each stage assumed negligible dispersive and mass transfer resistances. Changing the number of stages affected the sharpness of the calculated concentration profiles as a result of numerical dispersion. Therefore, the model is qualitative and is not intended to exactly reproduce the concentration profiles obtained from the fixed-bed and CAC experiments.

The system to be studied, in general, contains five exchangeable components: three amino acid cations, sodium, and hydrogen; as well as five coions: three amino acid anions, the hydroxyl ion, and the chioride or sulfate ions. Considering the dynamics of a fixed-bed column (the steady-state behavior of a CAC unit may be immediately obtained from this via the $\theta=\omega t^{\prime}$ transformation), the 
differential material balances on each amino acid $\left(A_{1}, A_{2}\right.$, and $\left.A_{3}\right)$, sodium, and chloride are

$$
\begin{gathered}
\epsilon \frac{\partial C_{A_{1}}}{\partial t}+\rho_{b} \frac{\partial q_{A_{1}}}{\partial t}+u \frac{\partial C_{A_{1}}}{\partial z}=0 \\
\epsilon \frac{\partial C_{A_{2}}}{\partial t}+\rho_{b} \frac{\partial q_{A_{2}}}{\partial t}+u \frac{\partial C_{A_{2}}}{\partial z}=0 \\
\epsilon \frac{\partial C_{A_{3}}}{\partial t}+\rho_{b} \frac{\partial q_{A_{3}}}{\partial t}+u \frac{\partial C_{A_{3}}}{\partial z}=0 \\
\epsilon \frac{\partial C_{N a}}{\partial t}+\rho_{b} \frac{\partial q_{N a^{+}}}{\partial t}+u \frac{\partial C_{N a^{+}}}{\partial z}=0
\end{gathered}
$$

and

$$
\epsilon \frac{\partial C_{C l-}}{\partial t}+u \frac{\partial C_{C l-}}{\partial z}=0
$$

These may be solved subject to initial and boundary conditions:

$$
\begin{array}{llll}
\text { I.C. for } & t=0, & \text { all z: } & q_{A i}=C_{A i}=C_{N a}=q_{N a}=0 \\
\text { B.C. for } & z=0, & 0<t \leq t_{F}: C_{A_{i}}=C_{A_{i}}^{F}, \quad C_{N a}^{+}=0 \\
& & t>t_{F}: & C_{A_{i}}=0, \quad C_{N a}^{+}=C_{N a^{+}}^{D}
\end{array}
$$

The remaining variables, $C_{H^{+}}$and $C_{O_{H}-}$, and the concentrations of amino acid cations and anions can be determined from $C_{A_{1}}, C_{A_{2}}, C_{A_{3}}, C_{N_{2}+}$, and $C_{C_{1}-}$ using the condition of electroneuirality as illustrated in Sect. 4.4.1. The only further information required is the equilibrium relation between the solid and the liquid phase concentrations. As discussed earlier, to simplify the calculations, the separation factor for binary ion-exchange reactions was assumed to be constant and equal to the average separation factor $\bar{S}$ given in Table 4.3. This assumption provides a reasonable approximation of the uptake isotherms at zero chloride concentration as was shown in Figs. 4.9 and 4.10. Furthermore, the assumption is reasonable since the affinity for the resin of leucine was much higher than that of valine, while the valine-glutamic acid separation was not strongly dependent on the separation factor being primarily determined by the $\mathrm{pH}$ effects.

An additional assumption was also made that $C_{A}=$ for glutamic acid is zero. This is a good assumption if glutamic acid elutes when the $\mathrm{pH}$ is less than 9, 
and experimental results show this to be true. It was also assumed that liquidphase accumulation is negligible in comparison to the sum of the convective and solid-phase accumulation terms. From Eq. 4.37 and for our experimental conditions where $\rho_{b}=0.33 \mathrm{~g} / \mathrm{cm}^{3}, \epsilon=0.4, \Delta q_{i}>5.0 \mathrm{mmol} / \mathrm{g}$, and $\Delta C_{i}<0.12$ $\underline{\mathbf{M}}$, the liquid phase accumulation term $\left(\epsilon \frac{\partial c}{\partial t}\right)$ was less than $3 \%$ of the other terms. Assuming negligible liquid phase accumulation, Eqs. 4.41 through 4.45 were converted to ordinary differential equations by backward finite difference of the convection term. The resulting ordinary differential equations are

$$
\begin{aligned}
\frac{d q_{A_{1}}^{j}}{d t} & =\frac{u}{\rho_{b} \Delta z}\left(C_{A_{1}}^{j-1}-C_{A_{1}}^{j}\right) \\
\frac{d q_{A_{2}}^{j}}{d t} & =\frac{u}{\rho_{b} \Delta z}\left(C_{A_{3}}^{j-1}-C_{A_{3}}^{j}\right), \\
\frac{d q_{A_{3}}^{j}}{d t} & =\frac{u}{\rho_{b} \Delta z}\left(C_{A_{3}}^{j-1}-C_{A_{3}}^{j}\right) \\
\frac{d q_{N a}^{j}}{d t} & =\frac{u}{\rho_{b} \Delta z}\left(C_{N a^{+}}^{j-1}-C_{N a^{+}}^{j}\right)
\end{aligned}
$$

and

$$
C_{C l^{-}}^{j-1}=C_{C l^{-}}^{j}
$$

where $j$ represents the stage number. Numerical solution of these equations was accomplished by an Adams predictor-corrector method using the International Mathematical and Statistical Library (IMSL) libraries routine DGEAR. During the integration, once the resin phase concentration had been determined at stage $j$, the liquid phase concentration could be determined from the equilibrium relation.

The model requires (1) experimental determination of the equilibrium isotherms for evaluation of fitting parameters in the equilibrium model, and (2) one experimental separation so that the number of stages, $\mathrm{N}$, can be fitted to the experimental data. With the information from these two experiments and 
the appropriate process parameters, the model can qualitatively predict concentration Frofiles and trends in operating parameters such as loading, displacer concentration, feed concentration, and rotation rate, even at nonisotachic conditions. 


\subsection{RESULTS AND DISCUSSION}

\subsubsection{Fixed-Bed Results}

Fig. 4.12 shows the experimental and calculated product concentrations and $\mathrm{pH}$ exit profile for a feed consisting of $15.4 \mathrm{mM}$ glutamic acid, $15.0 \mathrm{mM}$ valine, and $16.2 \mathrm{mM}$ leucine. The feed was supplied for $75 \mathrm{~min}$, the displacer was 100 $\mathrm{mM} \mathrm{NaOH}$, and the superficial velocity was $3.1 \mathrm{~cm} / \mathrm{min}$.

As discussed earlier, the glutamic acid-valine separation was due largely to differences in the isoelectric points of these two amino acids (3.2 and 5.9, respectively). When valine was eluting from the column, the solution $\mathrm{pH}$ was higher than the isoelectric $\mathrm{pH}$ of glutamic acid. Hence, glutamic acid was negatively charged and excluded from the resin by the Donnan potential effect. Valine and leucine, on the other hand, have very similar isoelectric points. If the separation were due to differences in their isoelectric points, these two components could not be separated. The separation was thus due to the specific affinities of valine and leucine for the resin.

From Fig. 4.12 it is apparent that product concentrations of the separated components are much higher than the feed concentrations. The product concentrations at the profile's peaks for glutamic acid, valine, and leucine are approximately 90 to $100 \mathrm{mM}$. Isotachic conditions in the bed have been reached since there tends to be a flat region at the top of each experimental curve. For isotachic conditions, the product concentrations can be determined from the approximation model by Glueckauf. Using Glueckauf's method, the outlet product concentrations were determined to be $85 \mathrm{mM}$, for glutamic acid and valine and $96 \mathrm{mM}$ for leucine. Glueckauf's method, therefore, worked quite well in predicting these concentrations.

To understand the extent of how concentrated the product has become the solubilities of L-glutamic acid, L-valine, and L-leucine are given in Table 4.1. Note that glutamic acid in Fig. 4.12 was eluted at a concentration above the 


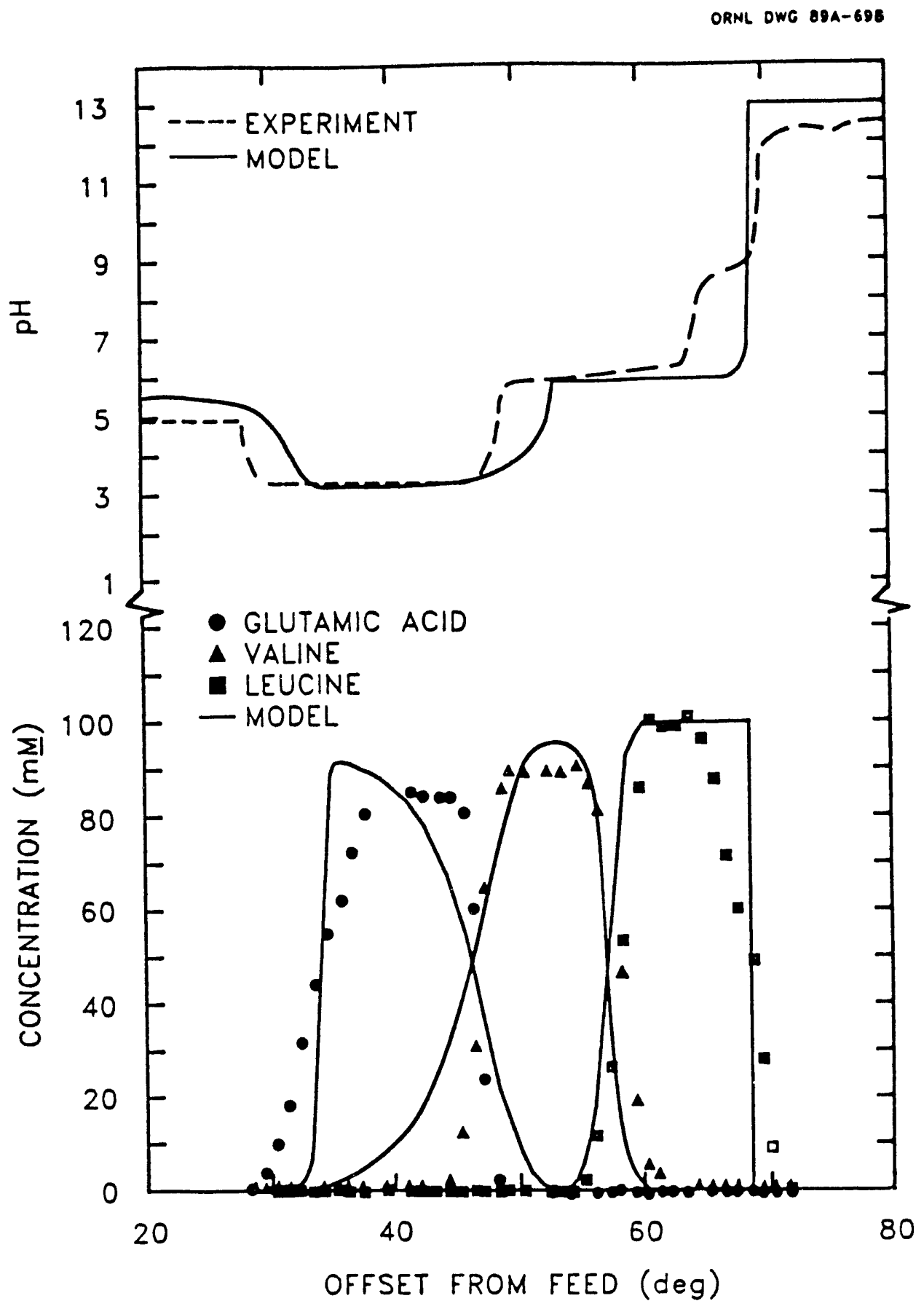

Fig. 4.12. Comparison of experimental and calculated fixed-bed displacement chromatographic separation. 
solubility limit in pure water. What is most surprising is that it did not crystallize and plug the bed. This is a very important operational consideration. Partridge (1949) also found that solutions above their solubilities could be obtained. Most likely, crystallization did not occur because of nucleation and kinetics limitation. Some of the eluted fractions, in fact, crystallized after a few days left undisturbed at room temperature.

The model calculations in Fig. 4.12 were based on constant separation factors and 100 equilibrium stages. The resulting concentration were profiles have been shifted 2 to $3 \%$ of the sodium breakthrough time to account for the assumption of negligible liquid-phase accumulation. Once shifted, it was easy to see that the experimental and theoretical values of the sodiudm breakthrough time agreed very well. This indicated that a sodium capacity of $5.6 \mathrm{mmol} / \mathrm{g}$, determined in batch experiments, was correct. Peak or "plateau" concentrations between model and experiment were equal, with the experimental values a bit higher. Most likely this was due to experimental error. The model also did a good job of predicting amino acid peak retention time, but trailing and leading edges of the peaks were not predicted as well. This reflects error due to the assumptions of local equilibrium and constant separation factors. Overall, the model did a good job of qualitatively predicting concentration and $\mathrm{pH}$ histories.

\subsubsection{CAC Results}

Displacement separations of glutamic acid, valine, and leucine were performed on the CAC. The feed typically contained a $25 \mathrm{mM}$ concentration of each amino acid, while the displacer contained $100 \mathrm{mM} \mathrm{NaOH}$. The feed arc was $45^{\circ}$, and the displacer and regenerator arcs were $90^{\circ}$ and could be varied if needed. Fig. 4.13 shows a typical CAC concentration profile. Like the fixed-bed run, the model calculations were based on constant separation factors. It was found that by doubling the number of stages, the predicted profiles could be slightly 
sharpened, with a corresponding increase in computer time by a factor of four. Since the model is qualitative in nature, 50 stages were used for all subsequent model calculations.

The calculated concentration profiles were matched to the experimental results by fitting the midpoint of the leucine trailing edge. This adjustment was typically $6^{\circ}$ or less and its necessity is attributed in part to neglect of the liquidphase accumulation and in part to accumulated experimental error.

Comparing Figs. 4.12 and 4.13, we find that the profiles are, indeed, similar. In the CAC experiment, simultaneous separation and concentration was achieved, as it was in the fixed-bed experiment.

Comparing the runs, it is evident that the $\mathrm{CAC}$ run also exhibited a characteristic plateau at the top of the concentration profile curve, indicating isotachic conditions. Here, as in the fixed-bed experiment, the amino acids eluted at their isoelectric point. From the $\mathrm{pH}$ profile in Fig. 4.13, the $\mathrm{pH}$ of the eluting solution when glutamic acid was displaced around 3.2 and climbed to about 6.0. These values were the isoelectric points of valine and leucine when they were being displaced. After valine and leucine were removed, the $\mathrm{pH}$ approached 13, which was the $\mathrm{pH}$ of the displacing $\mathrm{NaOH}$.

Both the experimental data and the theoretical calculations are in relative agreement. The model did a good job of predicting the retention angle and plateau concentration, although the agreement was not as good at leading and training edges because of neglected transport resistances and equilibrium nonidealities. This effect was also seen in the fixed-bed run.

In order to explore the operability of the CAC at different conditions, experiments were conducted which varied the displacer concentration, the amino acid feed concentration, and the rotation rate. Base conditions for these runs are shown in Table 4.5. All other operational parameters were kept constant, except those that were varied. At lower sodium concentrations it was necessary to extend the displacer arc so that an adequate amount of displacer was available for displacement. Figure 4.13 is an experiment at these base conditions. 


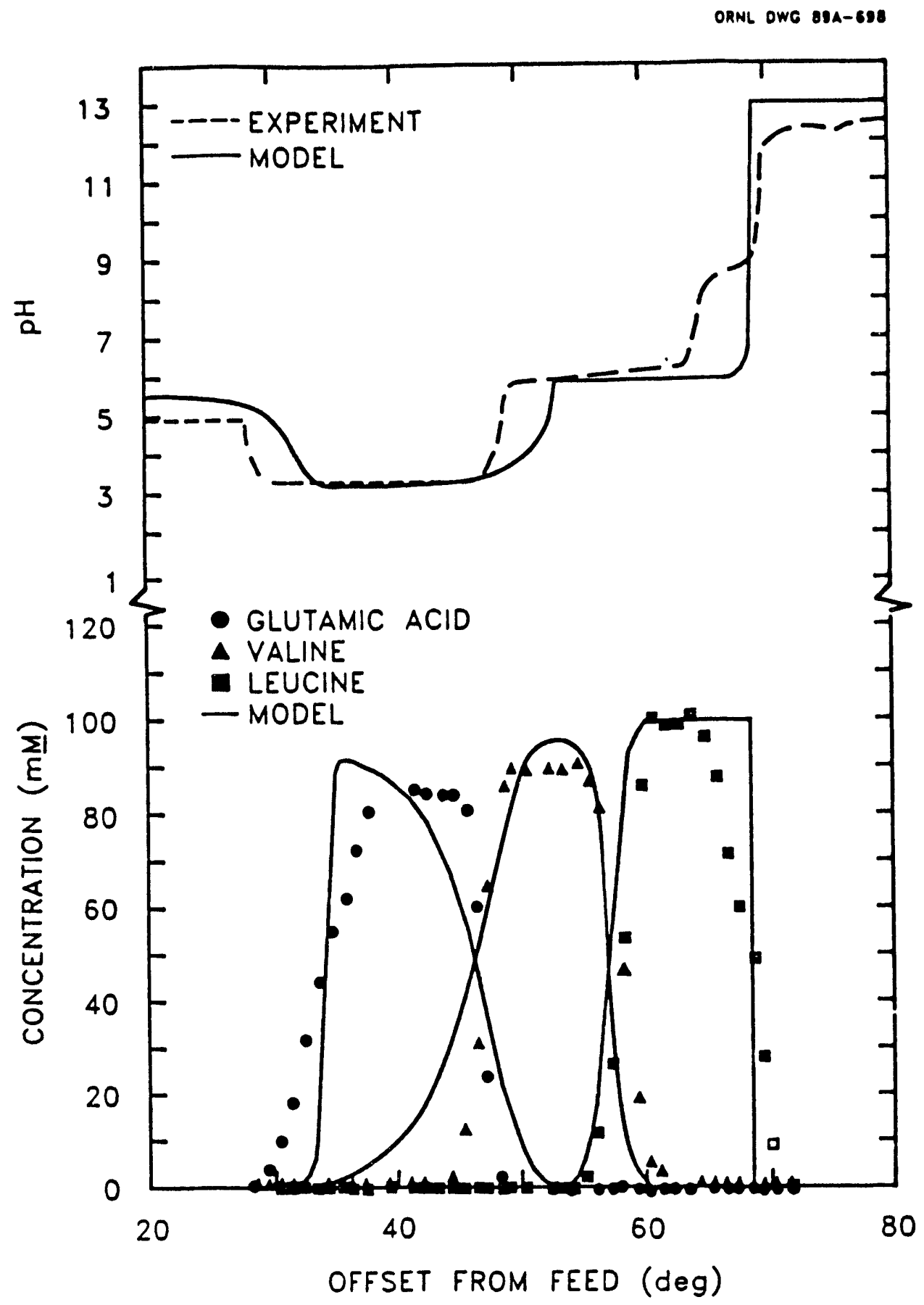

Fig. 4.13. Comparison of experimental and calculated CAC displacement chromatographic separation at base conditions (Table 4.5). 


\section{Table 4.5}

\section{Experimental Conditions}

\begin{tabular}{|c|c|c|}
\hline \multirow[t]{3}{*}{ Feed: } & $25.0 \mathrm{mM}$ & GLU \\
\hline & $25.0 \mathrm{mM}$ & VAL \\
\hline & $25.0 \mathrm{mM}$ & LEU \\
\hline Displacer: & $100 \mathrm{mM}$ & $\mathrm{NaOH}$ \\
\hline Regenerator: & $50 \mathrm{mM}$ & $\mathrm{H}_{2} \mathrm{SO}_{4}$ \\
\hline Feed Arc: & 45 & degrees \\
\hline Regenerator Arc: & $90-180$ & degrees \\
\hline Displacer Arc: & 90 & degrees \\
\hline Rotation Rate: & 30 & degrees/hr \\
\hline Superficial Velocity: & 2.9 & $\mathrm{~cm} / \mathrm{min}$ \\
\hline
\end{tabular}


In order to evaluate the separation performance of the system, a separation quality was defined in parallel to resolution (resolution as defined by Sect. 3 is meaningless here since the eluted peaks are adjacent even for ideal conditions). Considering a two-component ( $\mathrm{A}$ and $\mathrm{B}$ ) system, the components were fed to the column in equal amounts and the separated products we : collected in two pure fractions with a mixed-fraction mass $M_{A / B}$. The separation quality, $\eta_{A / B}$, is defined as

$$
\eta_{A / B}=1-\frac{2 M_{A / B}}{M_{A}+M_{B}}
$$

where $M_{A / B}$ is the mass of the mixed fraction, and $M_{A}$ and $M_{B}$ are the masses of components $\mathrm{A}$ and $\mathrm{B}$, respectively. The area under concentration profiles may also be used for the mixed and pure fractions since the areas under a concentration-time curve is proportional to the mass. If there is complete separation between components $\mathrm{A}$ and $\mathrm{B}, M_{A / B}=0.0$ and $\eta_{A / B}=1.0$. For the case of no separation, $M_{A}=M_{B}$ and $\eta_{A / B}=0.0$. Therefore, a separation quality of 1.0 indicates complete separation; a quality of 0.0 , no separation.

Evaluation of the product concentrations was performed by evaluating the peak concentration and the maximum average concentration at $90 \%$ recovery. Recovery of $90 \%$ was used so that peak tails would not drive the product concentration artificially low. The maximum average concentration means that the product cut was made to maximize the average concentration at $90 \%$. 


\subsubsection{Displacer Concentration Effects}

The product concentrations of the amino acids depend heavily on the displacer concentration. In the fixed-bed and CAC run (see Figs. 4.12 and 4.13), a displacer of $100 \mathrm{mM} \mathrm{NaOH}$ was used and the product concentrations reac' ed the theoretical limits predicted by Glueckauf's method. Here, a series of experiments on the CAC were made by varying displacer concentration. Figure 4.14 is an example of a run with a displacer concentration of $80 \mathrm{mM} \mathrm{NaOH}$. In this experiment, the peak concentrations approached $80 \mathrm{mM}$ and the model predictions agreed fairly well with the experiments. The effect that varying the displacer concentration had on the separation quality and on the peak and average concentrations was investigated. Displacer concentrations from 40 to 120 mM were used.

In Fig. 4.15, the separation quality is plotted as a function of the displacer concentration. The quality of the glutamic acid-leucine separation was equal to 1 over the entire range of displacer concentrations studied. Model calculations agree with these data. This, of course, results from the fact that valine was present between the two, making the separation quality very high. Experimental and theoretical calculations for valine-leucine and glutamic acid-valine are only in approximate agreement. In these cases, $\eta_{V A L / L E U}$ is smaller than predicted and $\eta_{G L U / V A L}$ is larger than predicted. Possible explanations are the assum $r$ tions of constant separation factors and no transport resistance. On the other hand, $\eta_{G L U / V A L}$ is larger than predicted, probably because of the assumption of constant separation factors.

This could not be an effect of mass transport because the model assumes none. If transport resistance were a relevant here, the model would predict a higher relevant separation quality, as in the valine-leucine separation quality. In either case, both theory and experiment show that separation quality is relatively independent of the displacer concentration. This allows experiments to be made with different displacer concentrations without having to worry about reduced 


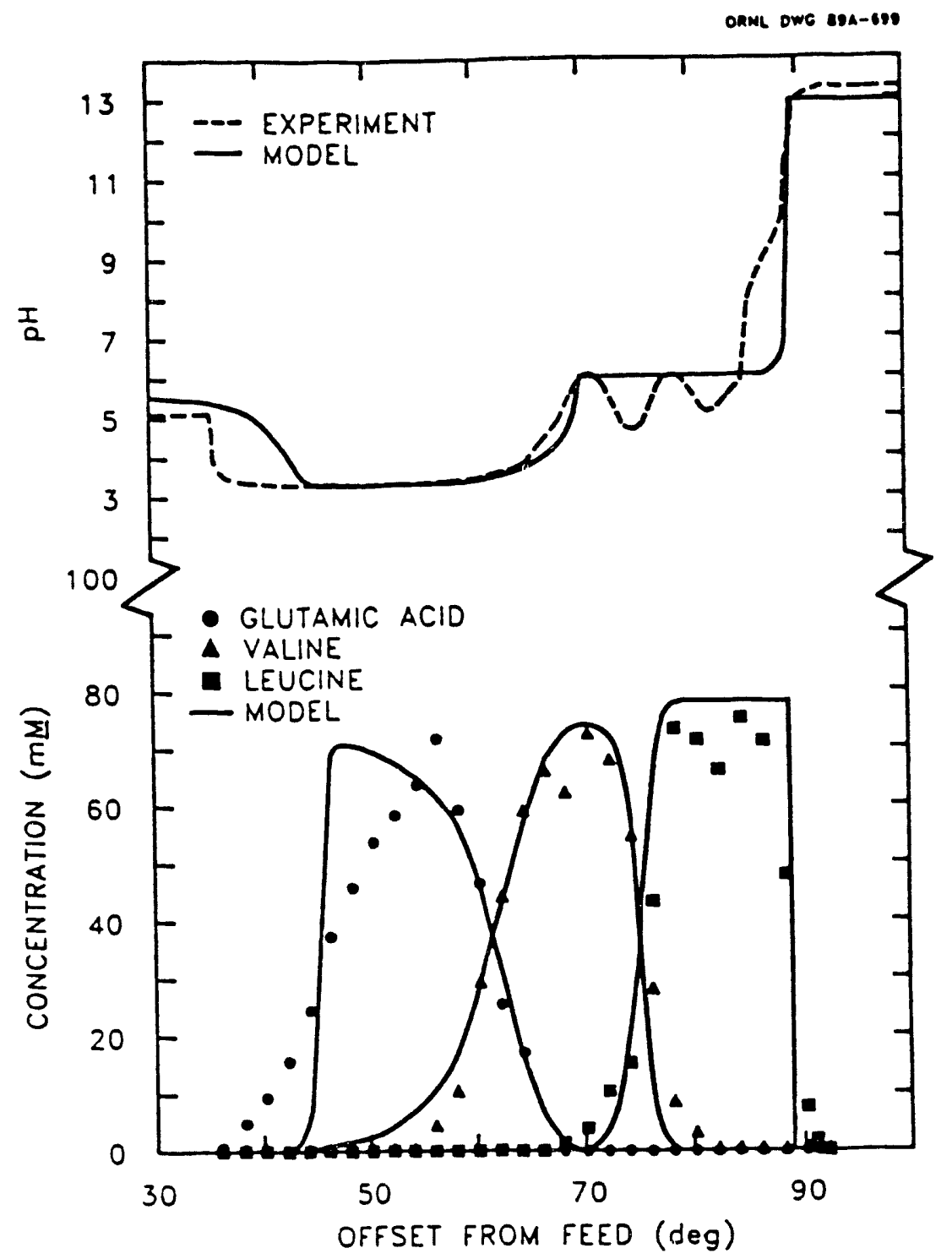

Fig. 4.14. Comparison of experimental and calculated CAC displacement chromatographic separation at base conditions (Table 4.5) with an $80 \mathrm{mM} \mathrm{NaOH}$ displacer concentration. 


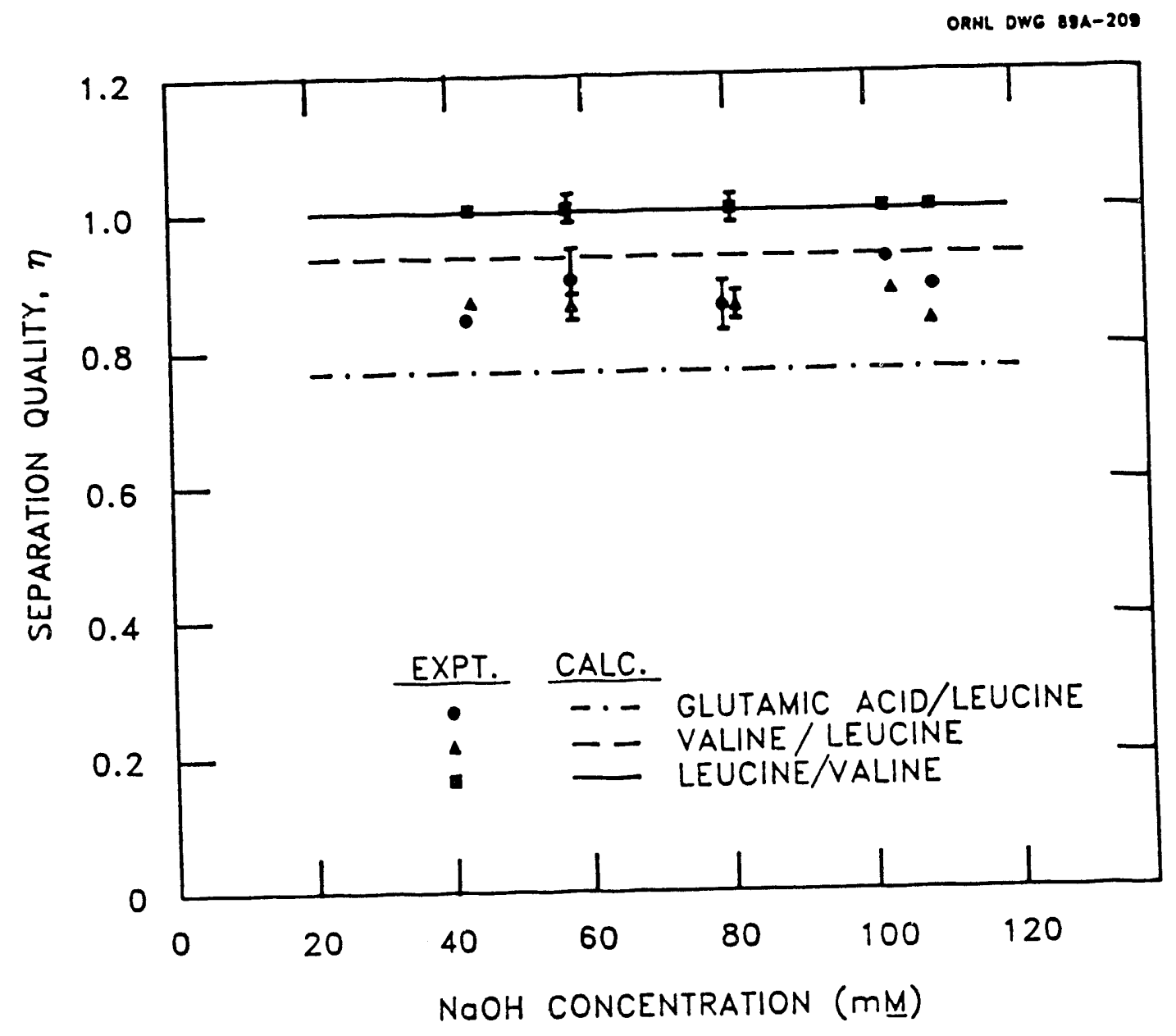

Fig. 4.15. Experimental and calculated values of the effect of $\mathrm{NaOH}$ displacer concentration on separation quality at base conditions (Table 4.5). 
separation efficiencies.

Figure 4.16 shows the effect of displacer concentration on average product concentration. Here, the model predictions for leucine are higher than the experimental data, while those of glutamic acid and valine agree quite well with the data. Figs. 4.17 and 4.18 illustrate the effects of displacer concentration on peak concentration. Fig. 4.17 uses the numerical model for prediction and Fig. 4.18 uses Glueckauf's method. Glueckauf's method tends to match the experimental data somewhat more closel- hecause the calculations were made from experimental equilibrium data ot from the assumed constant separation factor isotherm.

In general, increasing the displacer concentration increases the product concentrations, which can be adequately predicted in models assuming local equilibrium.

\subsubsection{Feed Concentration Effects}

Feed concentrations of 10,15 , and $25 \mathrm{~m} \underline{\mathrm{M}}$ of each amino acid were tested while keeping other base conditions constant at base values. The effects on separation quality and on average and peak concentration are shown in Figs. 4.19, 4.21, and 4.22 .

Figure 4.19 shows the effect that feed concentration has on separation quality. Experimental data and theoretical predictions do not quantitatively agree but the same trend is predicted. (The same number of stages is used here as in previously described runs.) An increase in the feed concentration at constant conditions increased the separation quality over the range studied. This is contrary to what would be expected in an elution technique where large loadings decrease the resclution. Figure 4.20 is an example of an experimental run and theoretical calculation at $10 \mathrm{mM}$ concentrations each of glutamic acid, valine, and leucine. It is readily apparent from this figure that isotachic concentrations 


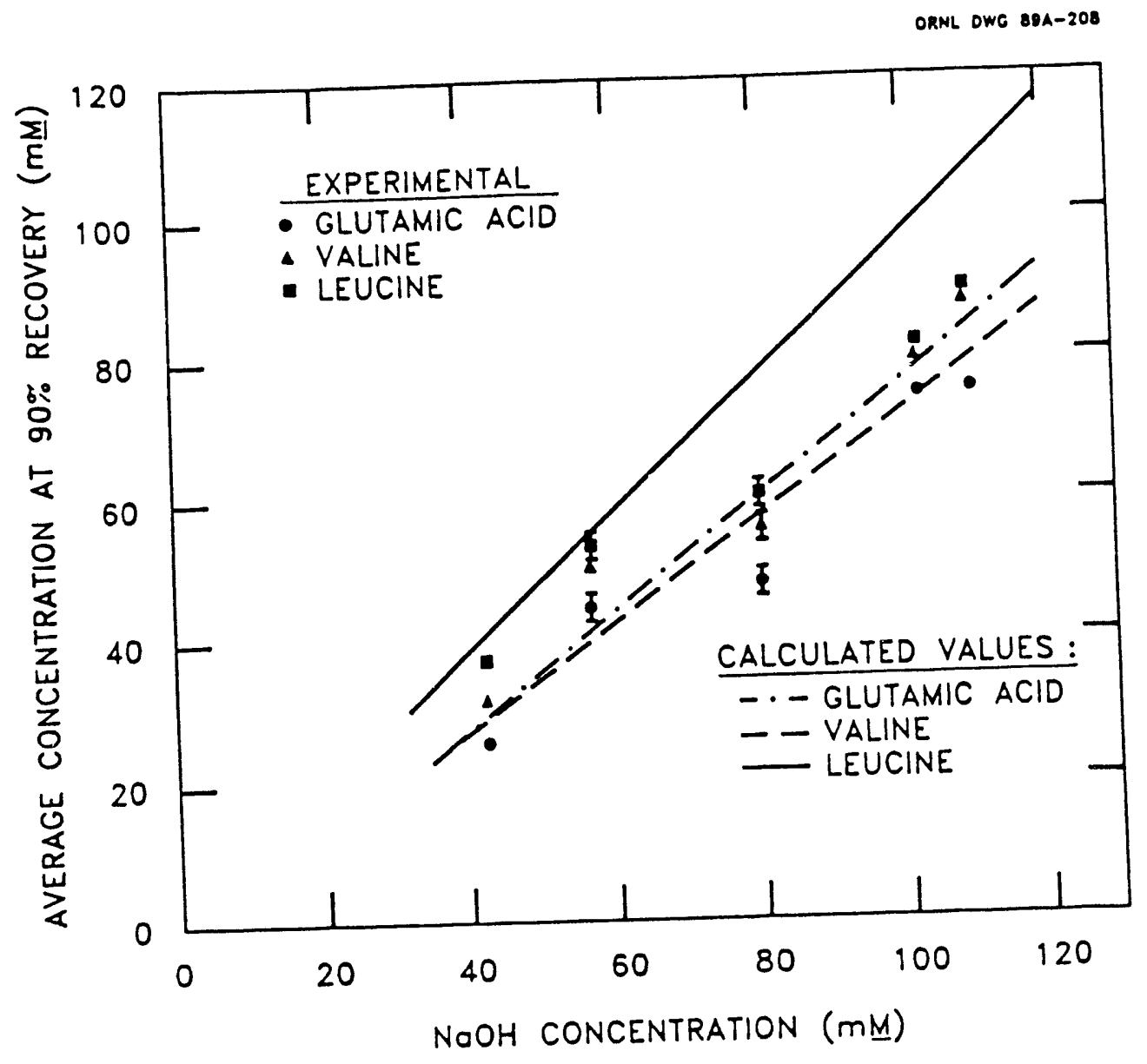

Fig. 4.16. Experimental and calculated values of the effect of $\mathrm{NaOH}$ displacer concentration on average concentration at base conditions (Table 4.5). 


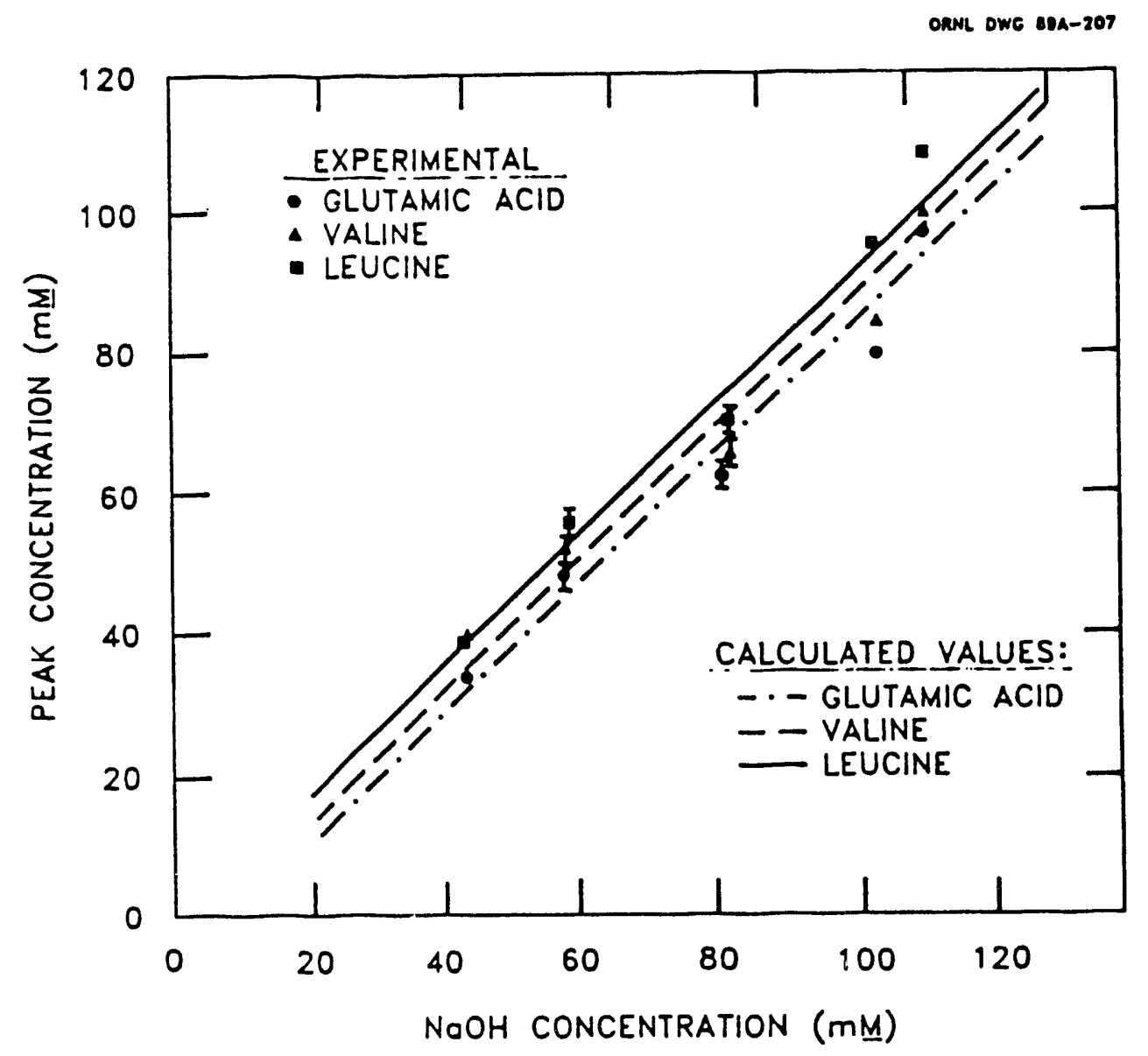

Fig. 4.17. Experimental and calculated values of the effect of $\mathrm{NaOH}$ displacer concentration on peak concentration at base conditions (Table 4.5). 


\section{ORNL DWG 89A-716}

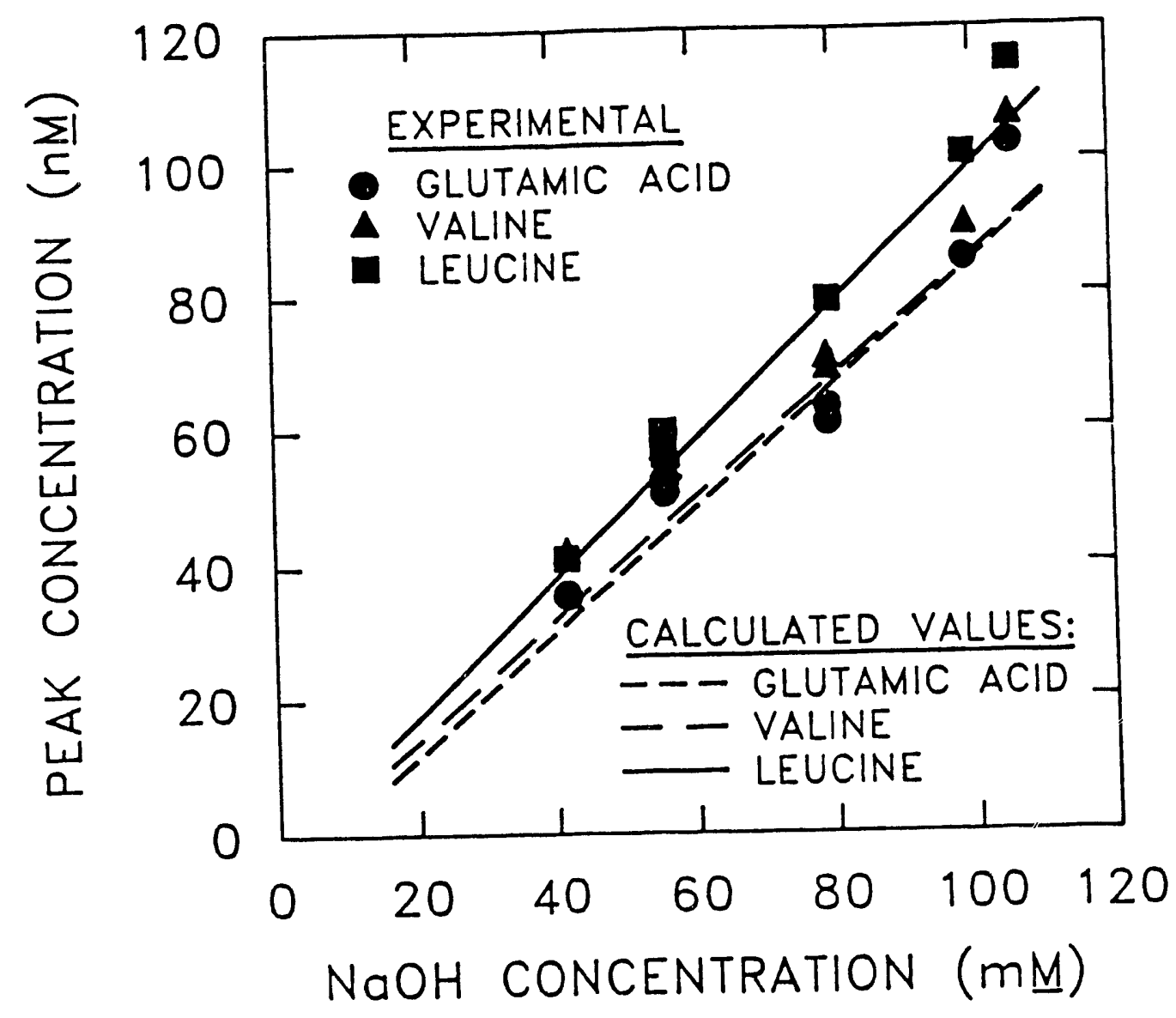

Fig. 4.18. Experimental and calculated values of the effect of $\mathrm{NaOH}$ displacer concentration on peak concentration using Glueckauf's method. 
ORNL DWG 2OA-20SR

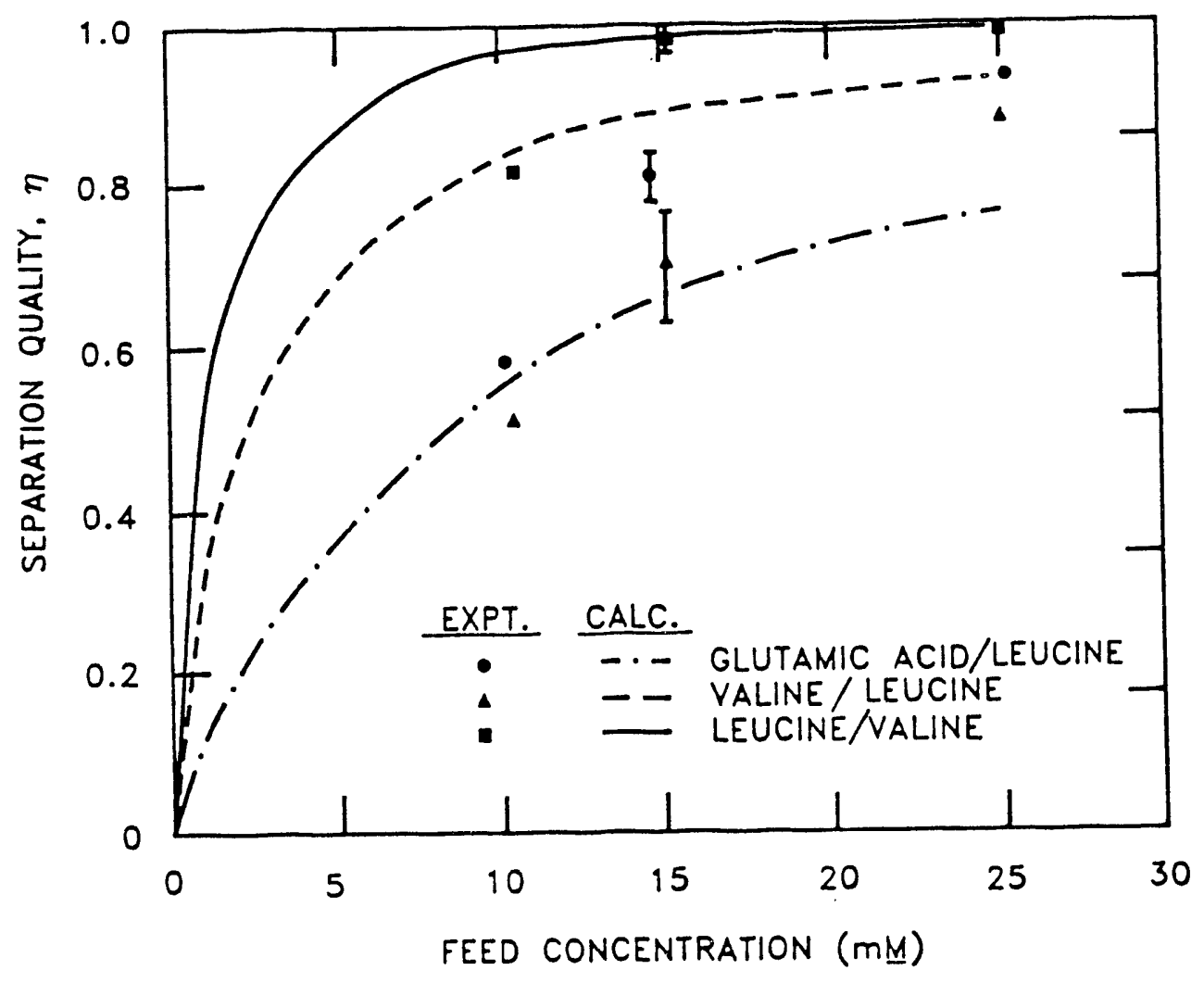

Fig. 4.19. Experimental and alculated values of the effect of feed concentration on separation quality at hith uliditions (Table 4.5). 


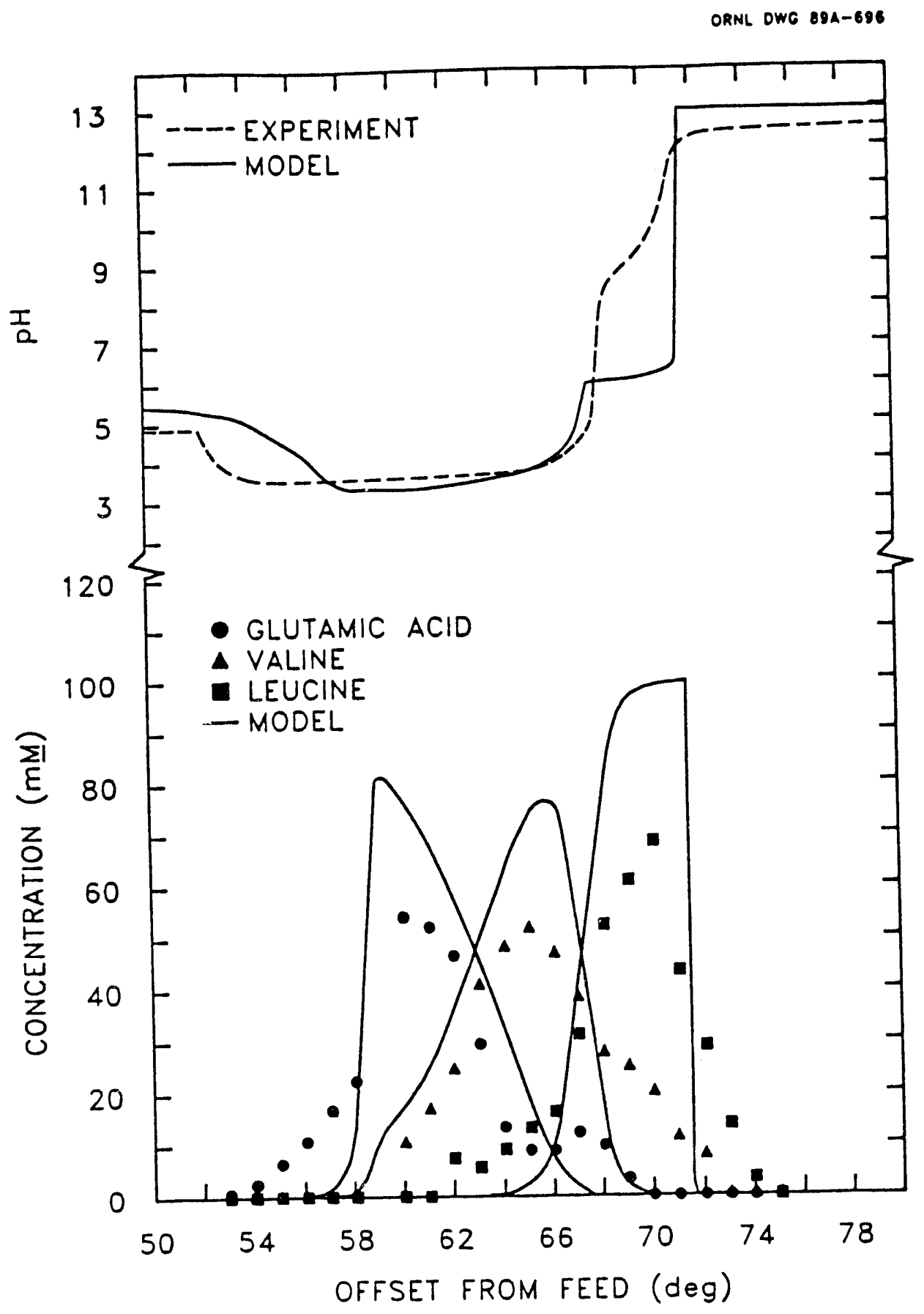

Fig. 4.20. Comparison of experimental and calculated CAC displacement chromatographic separation at base conditions (Table 4.5) with a $10 \mathrm{mM}$ feed concentration. 


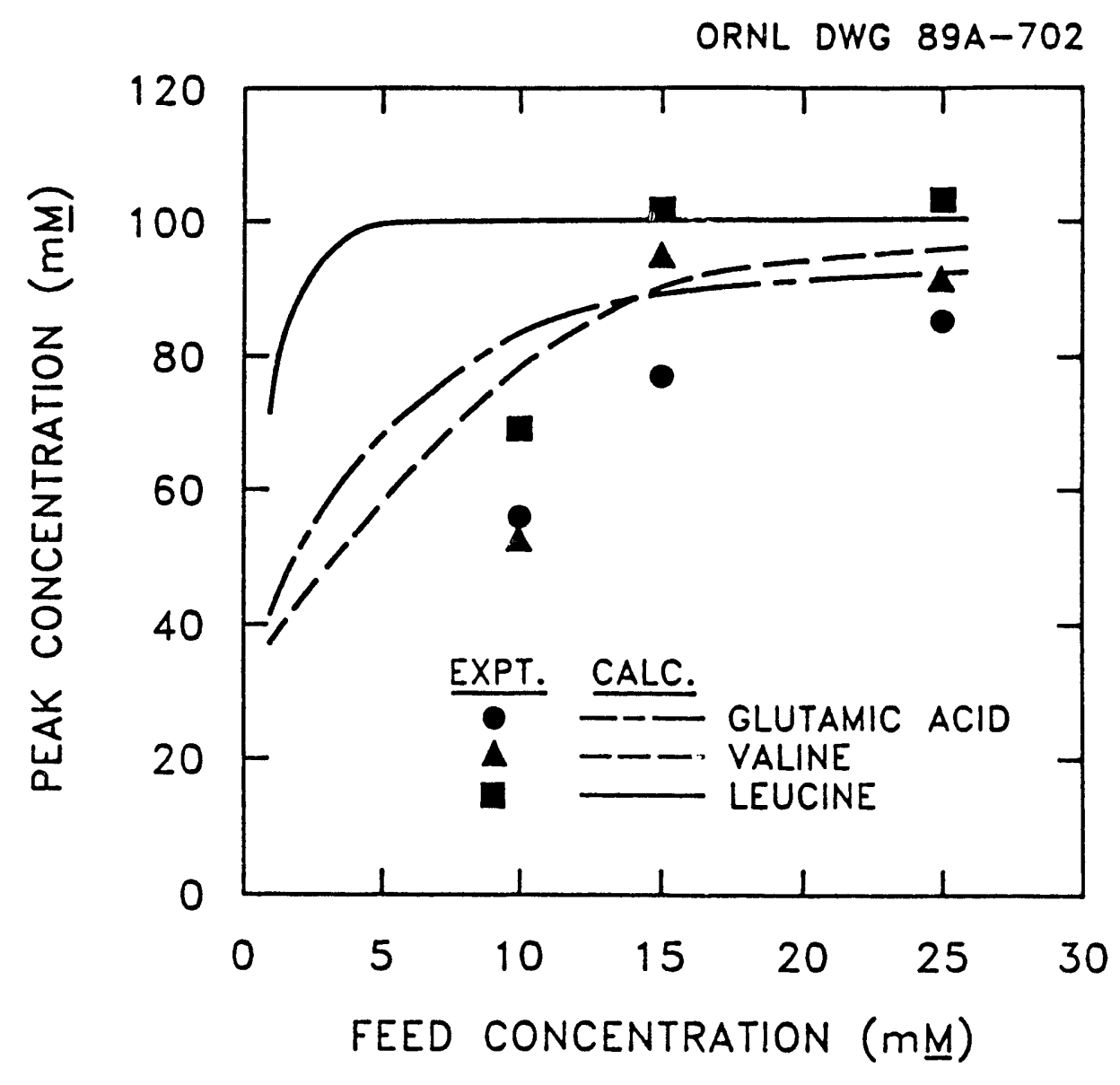

Fig. 4.21. Experimental and calculated values of the effect of feed concentration on peak concentration at base conditions (Table 4.5). 


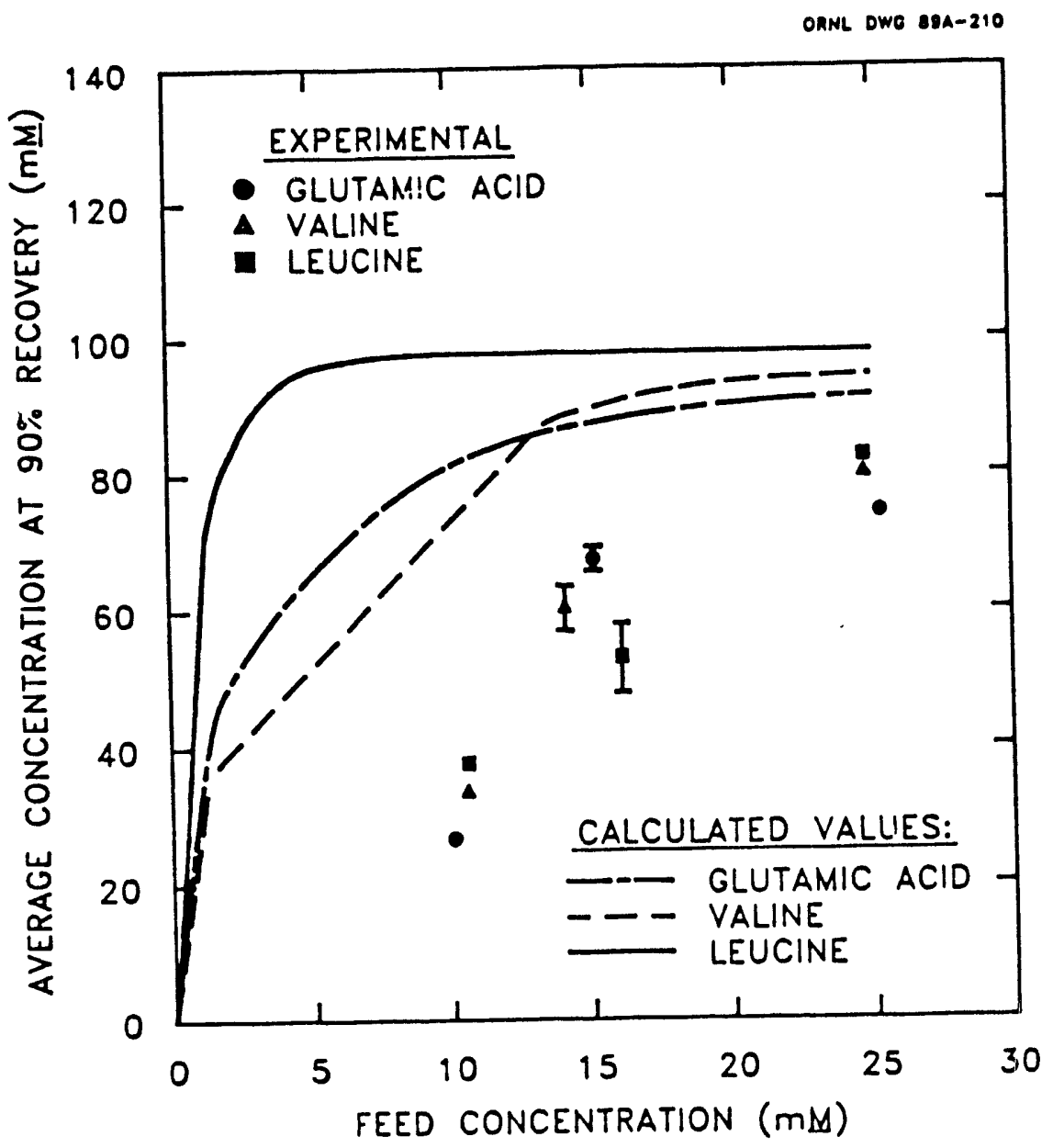

Fig. 4.22. Experimental and calculated values of the effect of feed concentration on average concentration at base conditions (Table 4.5). 
have not been reached. This is indicated by the dispersed nature of the experimental peaks, lack of boundary sharpness, and absence of plateau regions. By increasing the loading, or feed concentration in this case, isotachic conditions are reached sooner. This implies that in each bed there is a minimum loading required to attain isotachic conditions. The upper limit, of course, will be when the column becomes overloaded. On the other hand, at low to moderate loading, once isotachic conditions are reached, dispersive effects can cause dilution. Therefore, there must be an optimum loading that is neither too small nor too large. In practice, there is a range between overloaded conditions and nonisotachic conditions where separation quality is relatively constant.

In Figs. 4.21 and 4.22, the effects of feed concentration on peak and average concentrations are illustrated. Predictions and experin.ental data agree on a qualitative basis but not on a quantitative one. As discussed earlier, an optimum loading or feed concentration is required to reach isotachic conditions. These graphs lend support to this theory. For isotachic conditions the peak concentration should be independent of the feed concentration. At low feed concentrations, if isotachic conditions are not reached, the expected average and peak concentrations will be lower. Both the nodel and the experiments support the fact that at low feed concentrations, isotachic conditions may not be reached in short columns. A good test of the loading required might be conditions where peak and average concentrations in Figs. 4.21 and 4.22 do not significantly change with feed concentration. Model predictions of concentration profiles in these two plots tend to form : plateau like region at a feed concentration of $15 \mathrm{mM}$. However, it is easily seen that the experimental values have not, as yet, become constant, indicating that the model underestimates the amount of loading required to achieve isotachic conditions. 


\subsubsection{Rotation Rate Effects}

The effect of rotation rate on separation quality and on average and peak concentrations was investigated. Rotation rates of 20 and $30^{\circ} / \mathrm{h}$ were explored experimentally. Model predictions with rotation rates from 5 to $40^{\circ} \mathrm{h}$ were also made. The effect of rotation rates on separation quality is shown in Fig. 4.23. The separation quality between valine and leucine, $\eta_{V A L / L E U}$, is relatively constant but begins to decrease with increasing rotation rate. $\eta_{G L U / V A L}$, on the other hand, constantly increases with rotation rate until about $30^{\circ} / \mathrm{h}$ and then begins to decrease. By increasing the rotation rate, the amount of loading in any $1^{\circ}$ sector decreases. At high rotation rates, the "loading per degree" decreases therefore the column can no longer attain isotachic conditions. For example, while in the limit of no rotation, the feed section in the CAC behaves analogously to a fixed-bed column that is continuously fed with feed mixture. The effluent from a column with no rotation will have a separation quality of zero and peak and average product concentrations equal to their feed concentrations. If rotation rate is too low, the amino acid feed elutes from the column before the displacer is applied and does not receive the full benefit of the displacer solution. Fig. 4.24 is a model calculation at base conditions with a $10^{\circ} / \mathrm{h}$ rotation rate.

We note that the leucine peak has acquired a plateau, reaching isotachic condition. The profiles for glutamic acid and valine are, however, more complicated, exhibiting three plateaus and a low degree of separation. The leading glutamic acid plateau and the gradual rising of valine from zero to about 40 $\mathrm{m} \underline{\mathrm{M}}$ result from the competitive adsorption of glutamic acid, valine, and leucine during loading of the feed onto the hydrogen form of the resin. The remaining transitions are caused by the interference with the displacer. Of course, had the bed been longer, the displacer wave would have overtaken the non-coherent transitions displayed here for glutamic acid and valine forming a fully resolved displacement train. The numerical model predicts that an absolute minimum rotation rate of $15 d^{\circ} / \mathrm{h}$ is required if the displacer is to have the desired effect. 


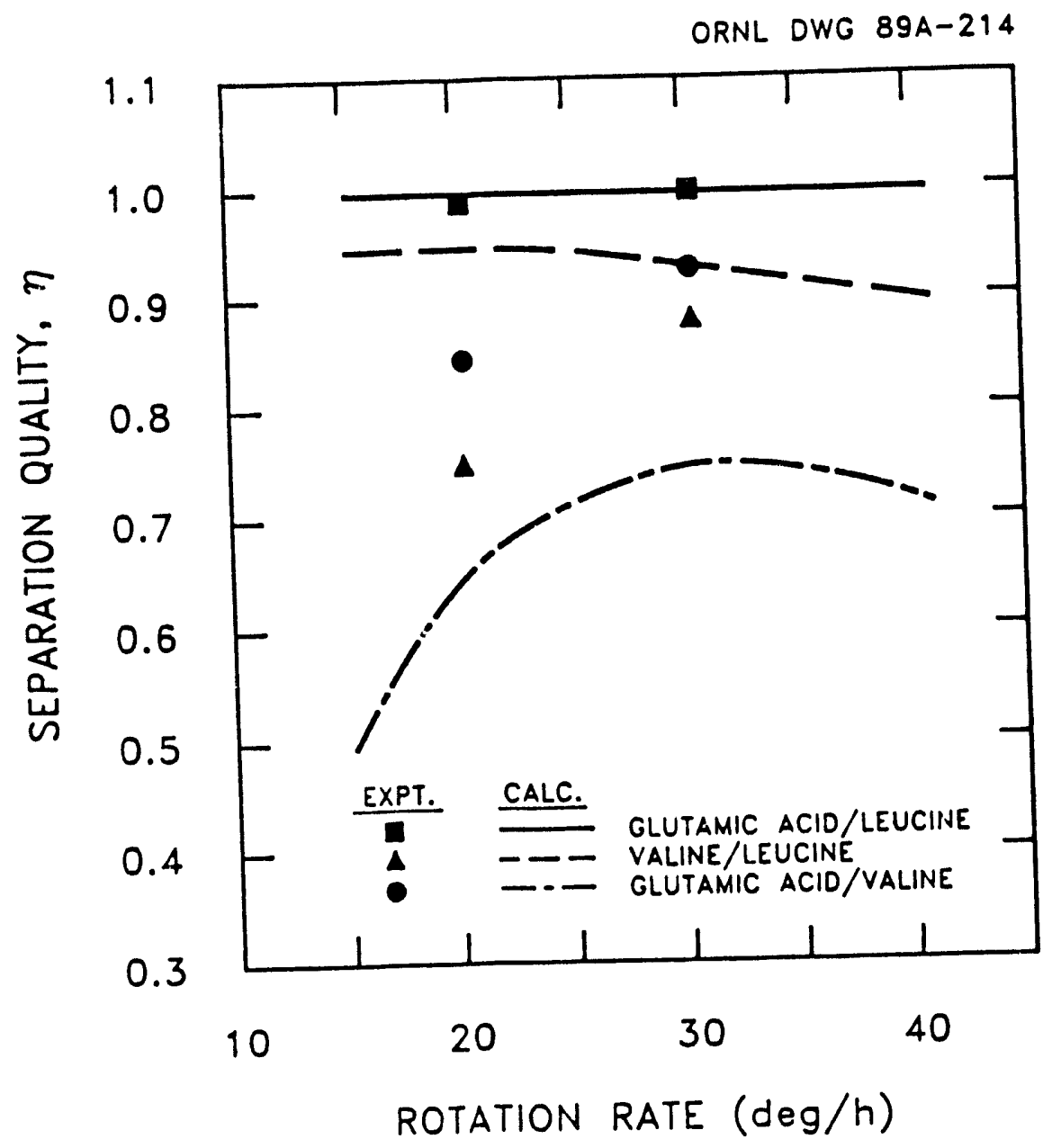

Fig. 4.23. Experimental and calculated values of the effect of rotation rate on separation quality at base conditions (Table 4.5). 


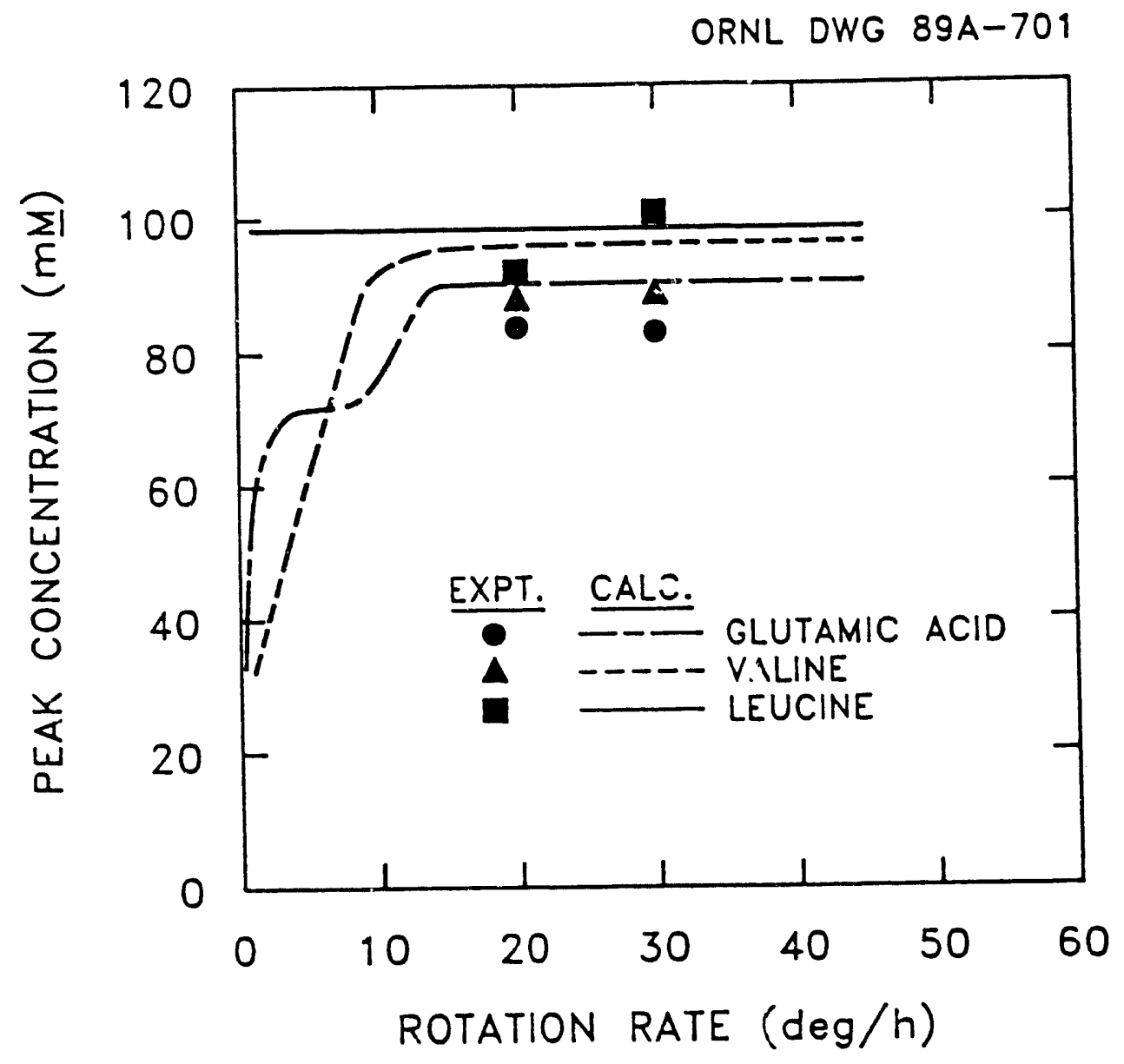

Fig. 4.24. Experimental and calculated values of the efiect of rotation rate on peak concentration at base conditions (Table 4.5 ). 
ORNL DWG 89A-574

EFFECT OF ROTATION RATE

10 DEGREES/h

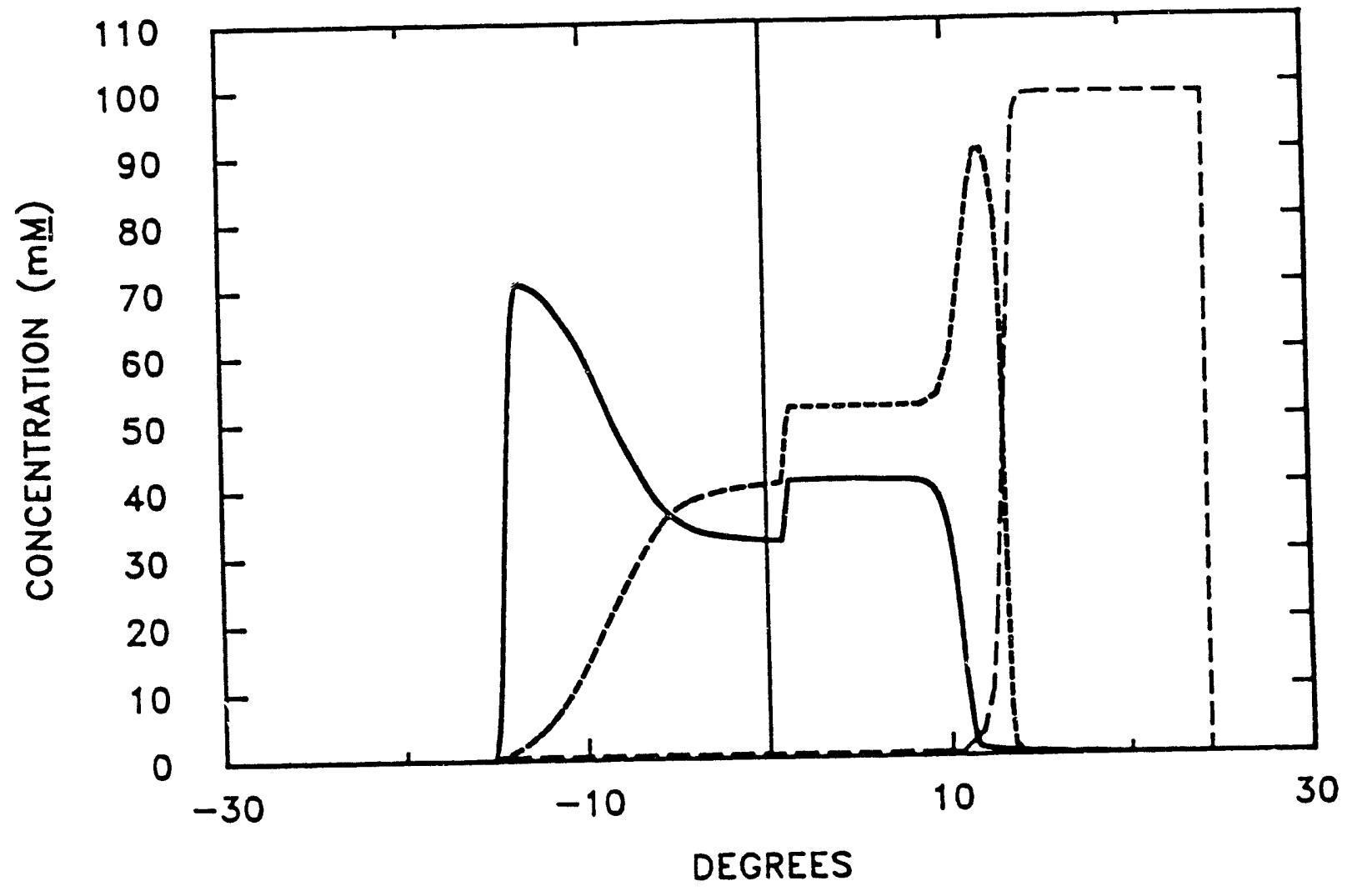

Fig. 4.25. Calculated values of glutamic acid $(0)$, valine $(+)$ and leucine (一) at a $10 \% / \mathrm{h}$ rotation rate at base conditions (Table 4.5 ). 


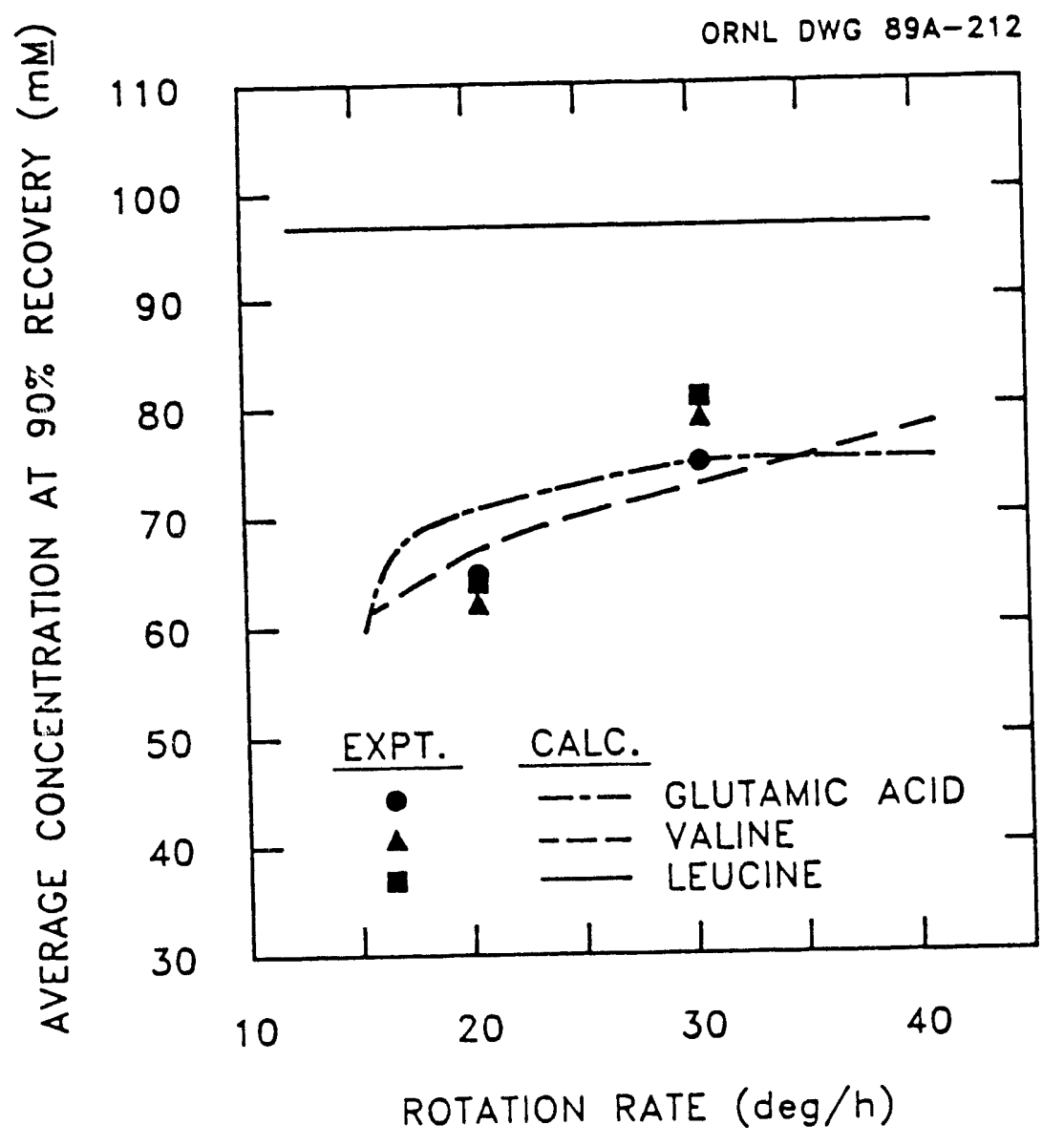

Fig. 4.26. Experimental and calculated values of the effect of rotation rate on average concentration at base conditions (Table 4.5). 
The effect on peak and average concentration is shown in Figs. 4.25 and 4.26. Peak concentration appeared to be independent of the rotation rate over the range studied. However, at low rotation rates, the average concentration of glutamic acid and valine slowly increased with increasing rotation rate.

At $30^{\circ} / \mathrm{h}$ the glutamic acid average concentration became fairly constant, as it would for isotachic conditions. The separation quality between glutamic acid and valine appeared to have a maximum at $30^{\circ} / \mathrm{h}$ indicating an optimum value of rotation rate for separation. There is, in summary, a minimum rotation rates that must be used to achieve isotachic conditions. If the rotation rate is too low, the feed concentration is not effected by the displacer. On the other hand, if the rotation rate is too high, the feed loading can be so low that isotachic conditions cannot be reached.

\subsubsection{Loading Application}

The effect of how the CAC was loaded was investigated theoretically. In principle, loading on the $\mathrm{CAC}$ can be increased by increasing the feed concentration or increasing the feed sector arc. Therefore, a series of calculations using the numerical model were performed which varied the feed concentration and the feed sector length at a constant value of loading. All other model parameters were kept constant. Loading was defined as

$$
L=C_{F} * Q_{F},
$$

where $C_{F}$ is the feed concentration and $Q_{F}$ is the feed how rate. Since the total flow rate was kept constant, an increase in the feed flow rate caused a proportional increase in the feed arc. For each value of loading, L, a numerical calculation was made assuming (1) a dilute feed with a wide feed arc, or (2) a concentrated feed with a narrow feed arc. These represent the two extremes. Feed concentrations were varied from 1.0 to $75 \mathrm{~m} \underline{\mathrm{M}}$ of each amino acid, with corresponding feed sectors from $135^{\circ}$ to $1.8^{\circ}$. Model calculations were made for 
both high and low loadings. At low to moderate loadings, the concentration profiles in both of the two cases described above were identical even if the system did not reach isotachic conditions. However, at overloaded conditions, the profiles were different. Concentration profiles at constant loading at overloaded conditions are shown in Fig. 4.27. An angle of $0^{\circ}$ corresponds to the beginning of the displacer sector. A comparison of the two predictions clearly shows that at angles greater than 0 (after the displacer sector), the two concentration profiles are identical but at angles less than 0 (before the displacer sector), the concentration profiles are different. At a high feed concentration and narrow feed arc (upper graph), a peak product concentration of $220 \mathrm{mM}$ is achieved, while at a low feed concentration and wide feed arc (lower graph), a peak concentration of $80 \mathrm{mM}$ was reached. Even though both profiles indicate no separation of glutamic acid and valine, the former attained a concentration three times that of the latter and, in some cases, might cause crystallization within the column.

Overloaded conditions where separation cannot be achieved is, of course, undesirable but it is important to be aware that at overloaded conditions, how the column is loaded can be as important as how much is loaded. Fortunately, wide feed sectors fed with low-concentration feed mixtures do not exhibit this problem. An amino acid experiment at high loading at the base conditions in Table 4.6 was performed with a $75^{\circ}$ feed sector. Experimental and theoretical concentration profiles are shown in Fig. 4.28 along with calculations based on the equilibrium stage model with 50 stages. While experimental and calculated profiles for leucine are in excellent agreement, there seems to be considerable discrepancy for glutamic acid and valine indicating that a more accurate description of equilibrium and mass transfer resistances is required for a more exact, quantitative comparison. More importantly, however, the experimental results, which actually yielded a better separation than predicted by the model, demonstrate operation of the $\mathrm{CAC}$ at high throughput. For these conditions the feed flow rate was about $61 \mathrm{~mL} / \mathrm{min}$ and the feed concentration $25 \mathrm{mM}$ for each amino acid. Three essentially pure product fractions contairing approximately 


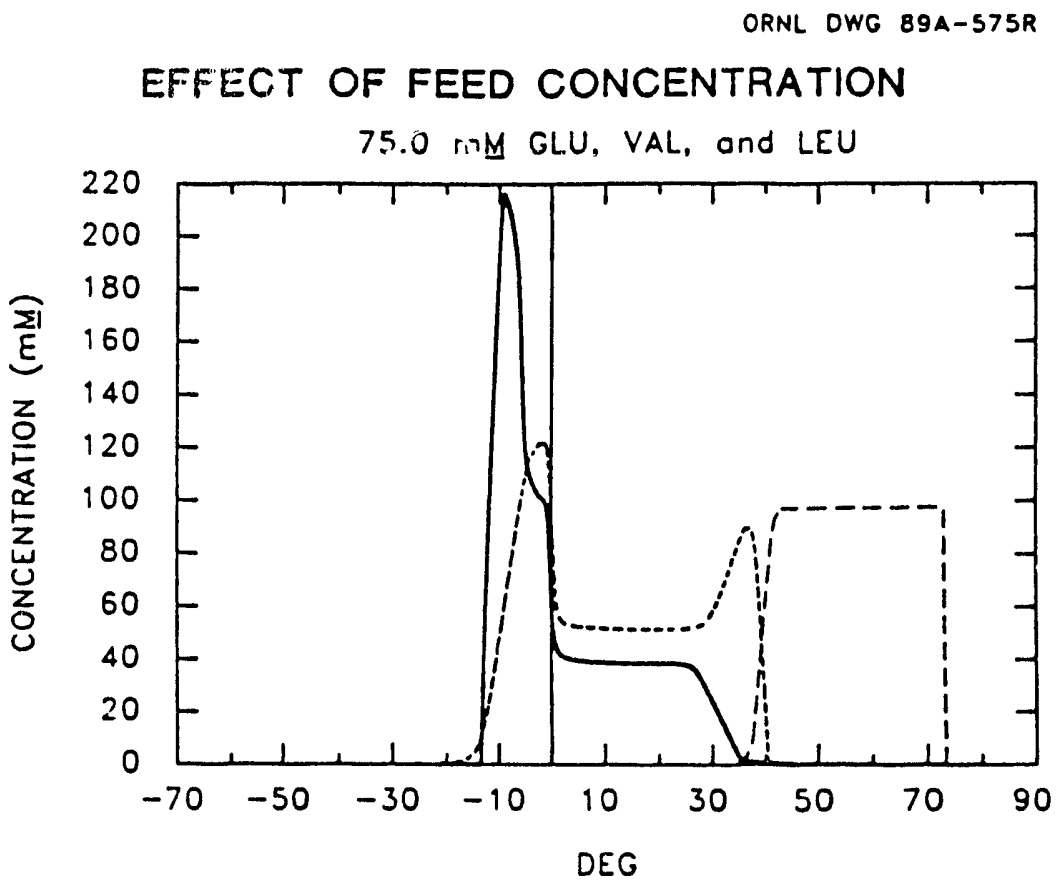

ORNL DWG 89A-576R

\section{EFFECT OF FEED FLOWRATE (OR FEED ARC)}

$112.5 \mathrm{~cm}^{3} / \mathrm{min}$ FEED (OR $135^{\circ} \mathrm{ARC}$ )

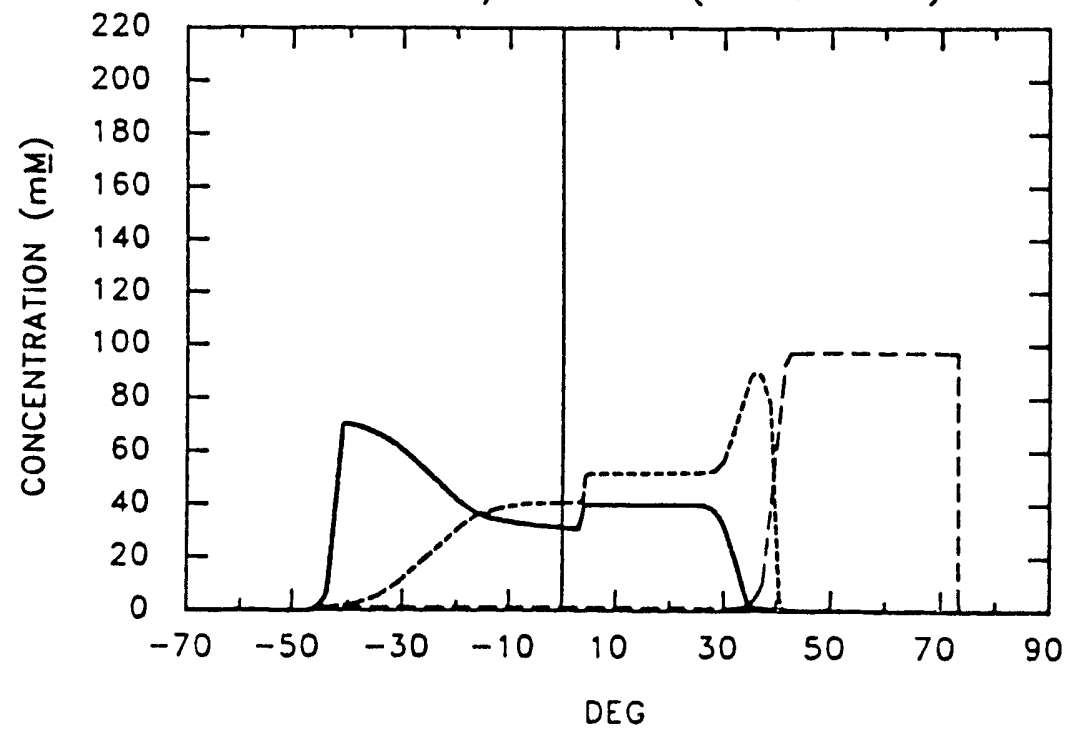

Fig. 4.27. Calculated values of glutamic acid $(0)$, valine $(+)$ and leucine $(-$ ) at the same loading with a concentrated feed narrow feed sector (upper graph) and a dilute-feed wide feed sector (lower graph) at base conditions (Table 4.5) 
$90 \%$ of each amino acid fed could be recovered from this run at an average concentration of about $80 \mathrm{mM}$.

\subsubsection{Nonbasic Displacer}

A comparison was made between the numerical model at the base conditions of Table 4.5 the (100 mM NaOH displacer) and conditions using a $100 \mathrm{mM}$ $\mathrm{NaCl}$ displacer. The resulting concentration profiles are shown in Fig. 4.29. This figure indicates that $\mathrm{NaOH}$ is a much more efficient displacer.

With the $\mathrm{NaOH}$ displacer, the components exited at their isoelectric $\mathrm{pH}$ and the solution $\mathrm{pH}$ approached the $\mathrm{pH}$ of the displacing solution once the amino acids had been completely eluted from the column. If $\mathrm{NaCl}$ is used as a displacer, the sodium ions exchanged with hydrogen, driving the column $\mathrm{pH}$ down while maintaining the amino acids in their cationic form. In the cationic form they are not excluded from the resin and so they are able to compete for ion-exchange sites with the other components. Glutamic acid and valine do not compete favorably with sodium, so they were somewhat displaced in front of the sodium band. However, they eluted from the column at a much lower concentration than when using a $\mathrm{NaOH}$ displacer. Leucine, on the (ther hand, competes favorably with sodium for ion-exchange sites and eluted from the column as a peak mixed with those ions. In many ways, this type of operation is similar to amino acid elution chromatography with buffer solutions since the $\mathrm{pH}$ remains relatively constant throughout the ion-exchange process.

\subsection{SUMMARY AND CONCLUSIONS}

Displacement separations of a glutamic acid-valine-leucine solution have been successfully demonstrated, for the first time, in the CAC. The product concentrations of the three amino acids could be determined by assuming local 


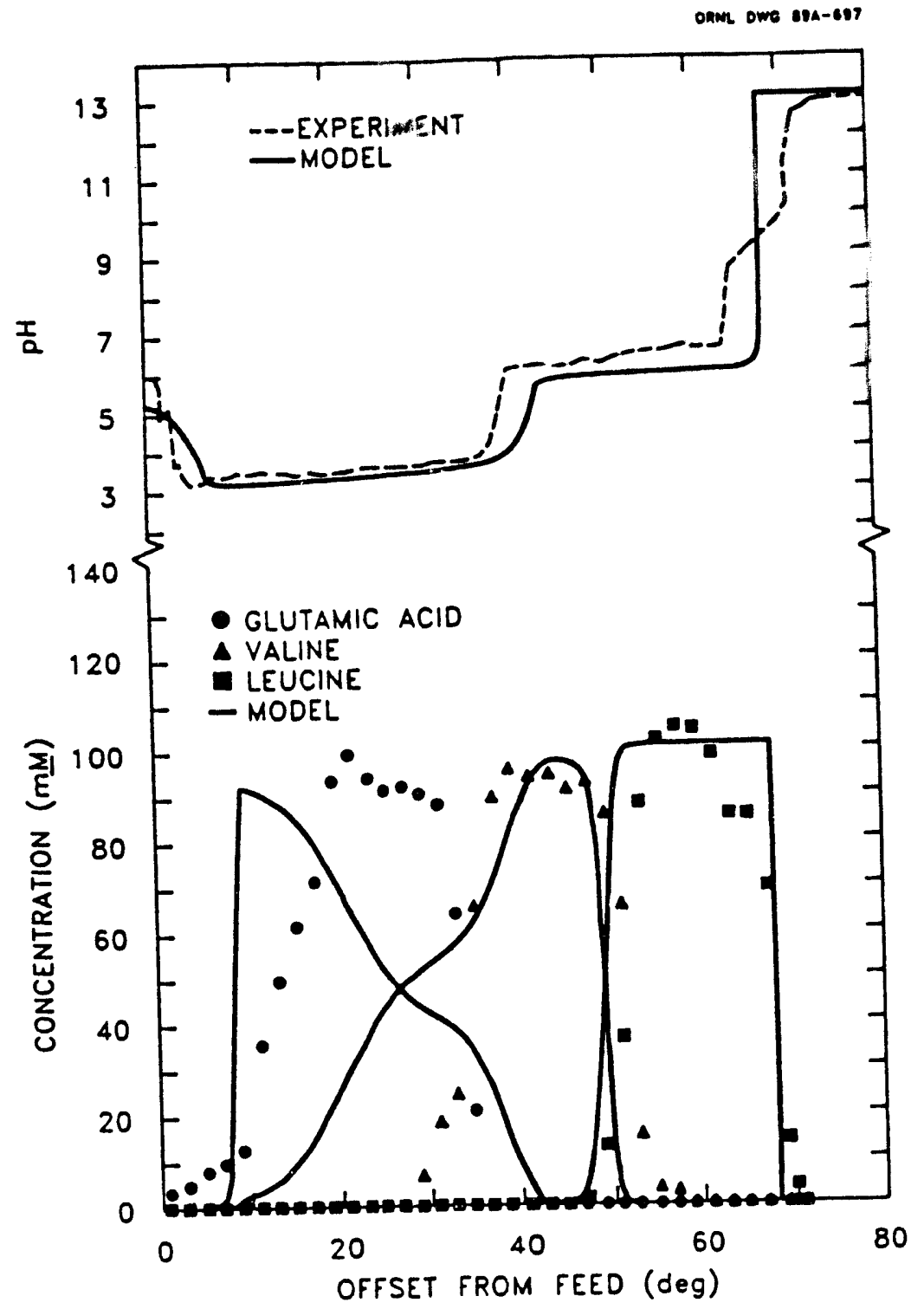

Fig. 4.28. Comparison of experimental and calculated CAC displacement chromatographic separation at base conditions (Table 4.5 ) with a $75^{\circ}$ feed arc. 

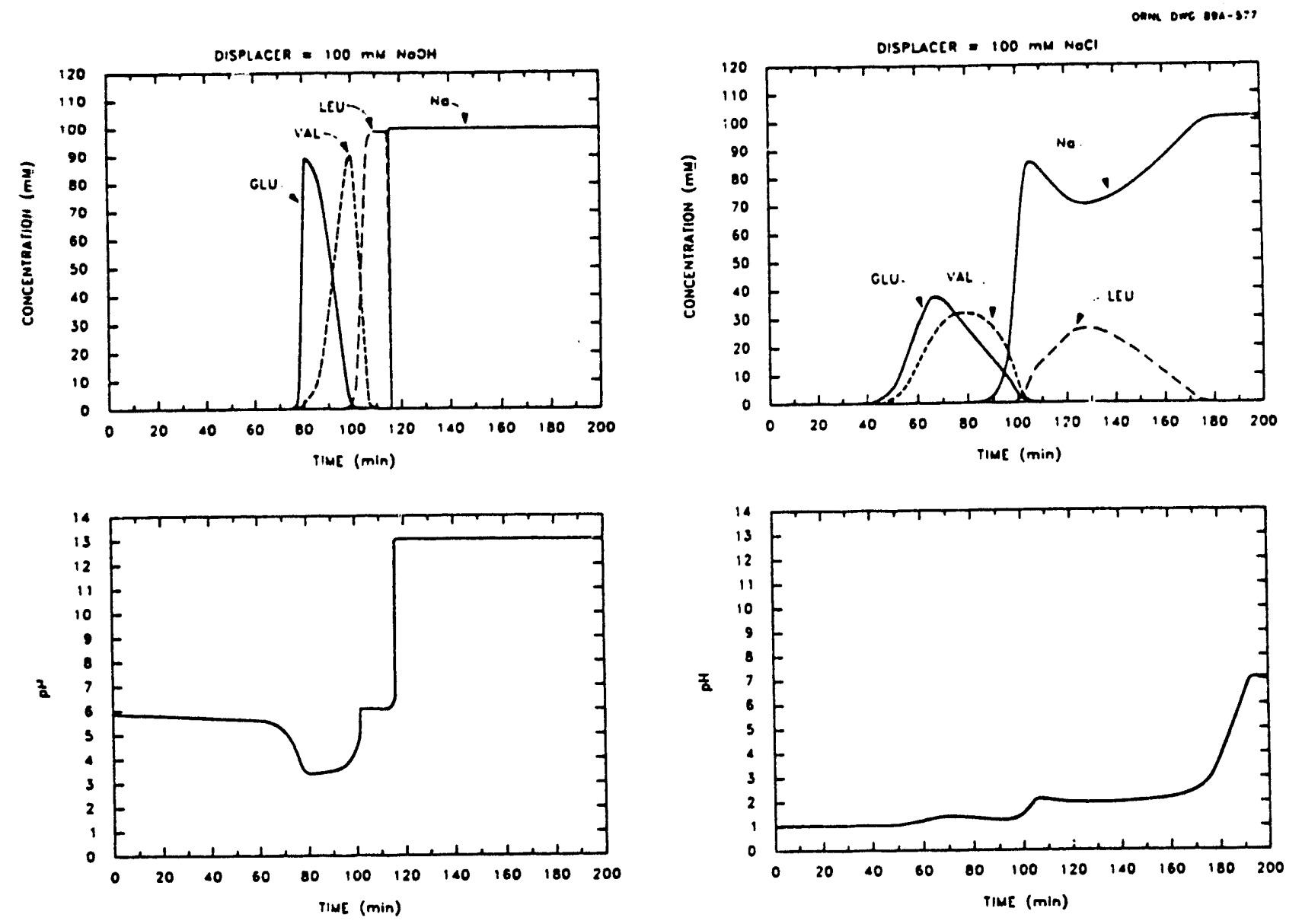

Fig. 4.29. Comparison of model predictions for fixed-bed displacement chromatographic separations at base conditions (Table 4.5) with a $100 \mathrm{~m} \underline{\mathrm{M}}$ $\mathrm{NaOH}$ and a $100 \mathrm{mM} \mathrm{NaCl}$ displacer. 
equilibrium and using Glueckauf's method. The achievable product concentrations depended on the displacer concentration, and were independent of the feed concentration provided that isotachic conditions were attained. Product concentrations as high as four times the feed concentration were observed. Outlet concentrations above the solubilities of glutamic acid in water were achieved in both the fixed-bed and CAC experiments without crystallization within the bed. This amino acid later crystallized from collected fractions that were undisturbed for several days. This is ideal for a separation process: a low concentration mixture to be separated is fed to a column and exits, separated from the other components, with a very high concentration.

Glueckauf's method was used to predict isotachic peak concentrations and was very successful. However, in order to predict nonisotachic column trends, an equilibrium-stage model was solved numerically. This model assumed local equilibrium and used constant separation factors. Constant separation factors could be assurned because the glutamic acid-valine separation was due to rather large differences of their isoelectric points, while the valine-leucine separation was due to differences in the amino acid's specific affinity for the resin. In systems with similar isoelectric points and similar affinities, the assumption of constant separation factors may fail; therefore, the model predicts no separation. In this case, the model developed by Saunders (1988) needs to be used to model amino acid uptake. For the three amino acids used here, the assumption of constant separation factors was fairly successful.

There was general agreement between the model and the experiments in the case of isotachic conditions. The model also adequately described the peak concentrations, elution angles, and the sodium breakthrough. For nonisotachic conditions, the model tended to overpredict concentration profiles, giving them a more isotachic nature and higher values. It did, however, predict general trends in CAC operating parameters such as displacer concentration, feed concentration, and rotation rate. The numerical model also predicted nonisotachic and overloaded conditions. It was determined that there is a minimum loading re- 
quired in a displacement development experiment to attain isotachic conditions. For low to moderate loadings, it appears that it does not matter whether the column is loaded with a narrow or with a wide feed sector. However, if the column is overloaded, a wide feed sector with dilute feed is preferred. The rotation rate, the feed concentration, and the feed sector determine column loading. While the range of successful operating conditions appears to be broad, the right combination must be used. For example, for successful displacement chromatography, the rotation rate must be fast enough so that all of the feed is actually displaced.

In summary, displacement chromatography has been demonstrated on the $\mathrm{CAC}$ and product concentrations as high as four times the feed concentration have been observed. For successful displacement experiments, a minimum loading and a rotation rate are required to attain desired isotachic conditions. The method of displacement chromatography applied to the CAC allows truly continuous, simultaneous separations and concentrations to be performed while increasing the sorbent column capacity. 


\section{CONCLUSIONS AND RECOMMENDATIONS}

Applications of the CAC concept to three different separation systems have been investigated in detail.

The first system, the separation of industrial concentrated mixtures of sugars, was chosen as an example to highlight certain scaleup issues which are key in determining the industrial application of CAC. Emphasis was placed on a study of high feed loadings and high feed concentrations with an experimental, pilot-scale, CAC unit. The results of this study were rather promising: the CAC could be successfully operated with diluted industrial mixtures of sugars at throughputs as high as $180 \mathrm{~L} / \mathrm{d}$ of feed. These experiments verified the possibility of directly scaling up the design of a CAC unit from data obtained in a small, laboratory, fixed-bed chromatographic column only $0.95 \mathrm{~cm}$ in diameter. At high feed concentrations, up to about $700 \mathrm{~g} / \mathrm{L}$, the $\mathrm{CAC}$ unit was still operable although the separation performance was reduced. In these runs, the sugar feed viscosity was as high as 20 times that of the eluent, causing significant viscous fingering and dispersion problems. Interestingly, such viscosity-induced nonidealities in the CAC were found to be not worse than those occuring in a fixed-bed chromatograph for analogous conditions.

The second system that was investigated was the separation of dilute mixtures of metal ions using stepwise elution techniques. Here, multiple eluents flowed simultaneously through the $\mathrm{CAC}$ unit with little mixing. Operation in this mode provided greater specific throughputs, reduced product dilution, and added additional flexibility in the selection of operating conditions. The experimental work was carried out in a bench-scale CAC unit. Comparison of a mathematical model (based on the operation of a fixed bed) with the CAC results showed, again, that the CAC unit performed in a nearly ideal manner and that the possibility exists of scaling up directly from fixed-bed chromatographic data.

Finally, the third system investigated was the separation of mixtures of amino 
acids by displacement development. The CAC apparatus was modified to allow up to four fluid streams to flow concurrently through the annular bed. With this technique, separation and concentration of multicomponent mixtures could be obtained simultaneously on the truly continuous basis of CAC operation. Again, although an exact comparison of $\mathrm{CAC}$ and fixed-bed performance was not made, qualitatively both operations could be directly scaled up semiquantitatively from data obtained in static batch experiments.

These studies provide a considerable breadth of applications of the CAC concept and allow some generalizations:

1. With $\mathrm{CAC}$ units ranging in diameter from 12 to 18 inches $(30.5$ to $45.7 \mathrm{~cm})$ and in length from 20 to $110 \mathrm{~cm}$, the continuous separation performance was found to be essentially the same as the batchwise separation performance of a conventional fixed-bed chromatograph operated with analogous conditions. This was observed virtually for all systems and modes of operation studied experimentally, in spite of the fact that the effective hydrodynamic dispersion in the CAC was at most twice as large as that in fixed beds. Some exceptions to this "rule" may, however, exist in practice. First, if very small sorbent particle sizes $(1-10 \mu \mathrm{m})$ were used (such as in HPLC separations), hydrodynamic dispersion would acquire a more dominant role, because of the very small mass transfer resistances inherent to the very small particles. CAC performance with these particles would be somewhat less efficient than the equivalent column. Secondly, for rapidly equilibrating systems such as gas phase processes, where the role of mass transfer resistance is very small and hydrodynamic dispersion determines the separation performance even with large sorbent particle, the CAC's performance might be inferior to column chromatography.

2. A variety of operating modes are possible in CAC operation. The apparatus retains its relative mechanical simplicity in comparison with fixed-bed processes. No precisely timed valving systems for feed introduction and product removal are necessary. However, an adequate flow distribution system is re- 
quired, although this would not be any more complex than that required by a fixed-bed system of comparable capacity.

3. The chief advantages of CAC over fixed-bed operations are likely the simplicity of the apparatus and its truly continuous operational capabilities. The latter is especially significant when the chromatographic system is to be coupled to other continuous unit operations both upstream and downstream of the separation process. At the same time, however, as a continuous process, CAC loses some of the flexibility of conventional chromatography. As any other continuous steady-state operation, the CAC must be designed and optimized for a specific separation task. Changing the task or altering operating conditions would require redesign for optimum operation.

Further work on the CAC is recommended in the following areas:

1. The effects of sorbent particle size on hydrodynamic dispersion in the CAC should be investigated. Only $50 \mu \mathrm{m}$ particles were used in the study by Howard (1987). While this small size provides a stringent test of the effects of dispersion on separation performance because of the relatively low mass transfer resistance, there may be other phenomena associated with the use of larger particle sizes.

2. Viscous fingering was shown to have a large detrimentai effect on the separation performance of the scaled-up sugar system. No quantitative study of this phenomenon is currently available in the literature, either for $\mathrm{CAC}$ or for fixed-bed operations. Such information would have more general use than just for sugar systems.

3. Mathematical models were developed for the various experimental systems and modes of operation. These models were developed in such a way as to require a minimum of independently experimental information. Only the model for amino acid displacement require the "fit" of a (single) parameter to experimental concentration profiles. These models are very useful for design and approximate optimization of the process. More sophisticated models, however, should be developed to improve the predictive ability by accounting 
for the details of transport processes and equilibrium behavior of the various systems.

4. Finally, new applications should be investigated. Biotechnology, for instance, offers many interesting application opportunities. One example is the separation and purification of monoclonal antibodies produced in cell cuiture systems. These systems are often operated continuously and may benefit, from the development of a truly continuous separation system. Work in this area is currently in progress at the University of Virginia (UVa)as part of the continued UVa/ORNL collaborative research program. 


\section{BIBLIOGRAPHY}

Altgelt, K. H., Sep. Sci., 5, 777 (1970).

Barker, P. E. and Ganetsos, G., Separation Science and Technology, 22, 8-10 (1987).

Barker, P. E.; Ganetsos, G.; and Thawait, S.; "Development of a Link Between Batch and Semicontinuous Liquid Chromatographic Systems", Chem. Eng. Sci., 41, 2595 (1986).

Begovich, J. M.; Byers, C. H.; Canon, R. M.; Scott, C. D.; Sisson, W. G.; and Watson, J. S., "Continuous Chromatographic Separations Conference" at ORNL (Nov. 1982).

Begovich, J. M. and Sisson, W. G., Hydrometallurgy, 10, 11 (1983).

Begovich, J. M., Ph.D. Dissertation, The University of Tennessee, Knoxville, 1982.

Begovich, J. M.; Byers, C. H.; and W. G. Sisson, Sep. Sci. Technol., 18, 1167 (1983).

Bio-Rad Catalogue, April, 1987.

Blackburn, S., "Amino Acids and Amines," Zweig, G. and Sherman, J., eds., in Handbook of Chromatography, CRC Press, Inc., Boca Raton, Fl. (1983).

Bollenback, G. N., "Special Sugars," in Ki,k-Othmer Encyclopedia of Chemical Technology, 3rd ed., V21, Wiley, New York, NY, pp 944-48 (1983).

Butt, J. B., “Reaction Kinetics and Reactor Design”, Prentice-Hall: Englewood Cliffs, p. 274. (1980).

Byalyi, A. L. and Ganitskii, M. B., USSR Patent 257, 134 (1969).

Byers, C. H. and Holmes, J. M., An Economic and Energy Evaluation of the Replacement of Conventional Technology with Continuous Chromatography in the Production of High-Fructose Sugar (Oct. 1988).

Canon, R. M.; Begovich, J. M.; and Sisson, W. G., Sep. Sci. Technol., 15, 655 (1980).

Canon, R. M. and Sisson, W. G., J. Liq. Chromatogr., 1, 427 (1978).

Carta, G., Chem. Eng. Sci., 43, 2877 (1988).

Ching, C. B. and Ruthven, D. M., Can. J. Chem. Eng., 62, 398 (1984). 
Ching, C. B.; Ho, C.; and Ruthven, D. M., Ind. Eng. Chem. Research, 26, 1407 (1987).

Claesson, S., Disc. Faraday Soc., 7, 34-38 (1949).

Claesson, S., Ark. Kem. Mineral. Geol., 24A, 7, 1-7 (1946).

Claesson, S., Ark. Kem. Mineral. Geol., 20A, 3, 1-14 (1945).

CRC Handbook of Chemistry and Physics, 57th ed., (1976-1977).

Dinelli, D.; Polezzo, S.; and Taramasso, M., J. Chromatogr., 7, 477 (1962).

Doddrell, D. and Allerhand, A., J. Amer. Chem. Soc., 93, 11 (1971).

Dunnill, P.; Lilly, M. D., Biotechnology and Bioengineering Symposium, No. 3, Geden E. L., ed.; Wiley, New York (1972).

Dye, S., Undergraduate Thesis, University of Virginia, Charlottesville, 1988.

Eliel, E. L.; Allinger, N. L. Angyal; S. J.; and Morrison, G. A., Conformational Analysis, Interscience Publishers, New York, pp 351-452 (1965).

Eliel, E. L., "Stereochemistry of Carbon Compounds," McGraw Hill, New York, p 41 (1962).

Ettre, L. S., "Evolution of Liquid Chromatogrpahy: A Historical Overview", in High-Performance Liquid Chromatography: Advances and Perspectives, C. Horvath, ed., Academic Press, New York (1980).

Ettre, L.S., "Evolution of Liquid Chromatogrpahy: A Historical Overview", in Horvath, C. (Ed.), High-Performance Liquid Chromatography: Advances and Perspectives, Academic Press, New York (1980).

Fox, J. B.; Calhoun, R. C.; Eglington, W. J.; Nicholas R. A.; J. Chromatogr., 43, 48 (1969).

Ganetsos, G., J. Chromatogr., 411, 81 (1987).

Ganno, S.; Hamano, Y.; Kobayashi, J., and Masaki, T.; J. Chromatogr., 332, 275-82 (1985).

Ghim, Y. S. and Chang, H. N., Ind. Eng. Chem. Fundam., 21, 369 (1982).

Giddings, J. C., Anal. Chem., 34, 37 (1962).

Glueckauf, Discuss. Faraday Soc., 7, (1949). 
Goulding, R. W., J. Chromatogr., 103, 229 (1975).

Gronlund, F. and Andesen, B., Acta Chem. Scand., 20, 2663 (1966).

Hebeda, R. E., "Syrups," in Kirk-Othmer Encyclopedia of Chemical Technology, 3rd ed., V22, Wiley, New York, 499-510 (1983).

Heftmann, E., Chromatography, A Laboratory Handbook of Chromatographic and Electrophoretic Methods, 3rd ed., Van Noostrand, New York (1975).

Helfferich, F., Ion Exchange, McGraw-Hill, New York (1962).

Helfferich, F. and Klein, G., Multicomponent Chromatography, Marcel Dekker, New York (1970).

Hill, C. G., Chemical Engineering Kinetics and Reactor Design, Wiley, New York, NY (1977).

Howard, A. J., M.S. Thesis, University of Virginia, Charlottesville, 1987.

Howard A. J.; Carta G.; and Byers C. H.; Separation of Sugars by Continuous Annular Chromatography, ORNL/TM 10318 (1987).

Howard, A. J.; Carta, G.; and Byers, C. H.; Ind. Eng. Chem. Research, 27, 1873 (1988).

Hughes, G. J. and Wilson, K. J., J. Chromatogr., 242, 337-41 (1982).

Isbell, H. S. and Pigman, W. W., J. Res. Nat. Bur. Stand., 20, 773 (1938).

Kulkarni, V. G. and Whiteley, K. J., Textile Research Journal., 46, 372-74 (1977).

Lehninger, A. L., Biochemistry, Worth Publishers, Inc., New York, 277-298 (i977).

Malton, H. F. and Navratil, J. D., in Recent Developments in Separation Science, Li, N., ed.; CRC Press, 65-91 (1981).

Martin, A. J. P., Discuss. Faraday Soc., 7, 332 (1949).

Meister, A., "Biochemistry of the Amino Acids," Vol. I, Academic Press, New York (1965).

Mikes, O., Chromatographic and Allied Methods, Ellis Horwood Limited, Chichester (1979). 
Moore, J. C., Sep. Sci., 5, 723 (1970).

Moskvin, L. N.; Kozhin, S. A.; and Fleisher, A. Y., J. Appl. Chem. USSR., 44, 2056 (1974).

Moskvin, L. N.; Gumerov, M. F.; and Gorshkov, A. I.; J. Appl. Chem. USSR., 47, 1917 (1974).

Moskvin, L. N., Mozzhuklin, A. V.; and Tsaritsyna, L. G.; J. A spl. Chem. USSR., 30, 2056 (1975).

Murren, C., Stelling, D.; and Felstead, G.; J. Chromatogr., 115, pp 236-39 (1975).

Myers, A. L. and Byington, S., "Thermodynamics of Ion Exchange: Prediction of Multicomponent Equilibria from Binary Data," in Ion Exchange Science and Technology, Rodrigues, A. E., (eds.), NATO ASI, Series E., No. 107, Martinus Nijhoff Publ., Dordrecht (1986).

Novosad, J. and A. L. Myers, "Thermodynamics of Ion Exchange as an Adsorption Process," Can. J. Chem. Eng. 60, 500 (1982).

Partridge, S. M. and Brimley, R. C., Biochem., 51, 628 (1951).

Partridge, S. M. and Brimley, R. C., Biochem., 49, 163 (1951).

Pariridge, S. M. and Westfall, R. G., Biochem., 44, 418 (1949).

Partridge, S. M. and Brimley, R. C., Biochem., 44, 513 (1949).

Partridge, S. M., Biochem., 44, 521 (1949).

Partridge, S. M. and Brimley R. C., Biochem., 46, 334 (1950).

Partridge, S. M. and Brimley R. C., Biochem., 48, 313 (1951).

Partridge, S. M., Biochem., 44, 459 (1949).

Redman, D. G., J. Chromatogr., 104, 178-79 (1975).

Reeves, R. E., J. Am. Chem. Soc., 71, 215 (1949).

Reisman, H., "Amino Acids," in Encyclopedia of Chemical Processing and Design, Marcel Dekker, Inc., New York, 197-256 (1977).

Rhee, H. K.; Aris, R.; and Amundson, N. R., "On the Theory of Multicomponent Chromatography," in Trans. Roy. Soc., A267, 419-55 (1970). 
Robinson, G. W., J. Chromatogr., 111, 416-18 (1975).

Ruthven, D. M., "Principles of Adsorption and Adsorption Processes", Wiley, New York (1984).

Saunders, M. S., Ph.D. Dissertation, University of Virginia, Charlottesville, 1989.

Scott, C. D.; Spence, R. D.; and Sisson, W. G.; J. Chromatogr., 126, 381 (1976).

Shallenberger, R. S., "Sugar Structure and Taste," in Carbohydrates in Solution: Advances in Chemistry Series No. 117, ACS (1973).

Sherwood, R. K., Pigford, R. L., and Wilke, C. R., Mass Transfer, McGraw-Hill, New York, 1975, 548-91.

Siegell, J. H., Dupre, G. D., and Pirkle, Jr., J. C., Chem. Eng. Prog., 57-61 (Nov. 1986).

Snyder, L. R. and Kirkland, J. J., Introduction to Modern Liquid Chromatography, John Wiley and Sons, New York (1974).

Sussman, M. V., Astill, K. N., and Rathore, R. N. S., J. of Chromatogr. Sci., $12,91(1974)$.

Sussman, M. V., Rathore, R. N. S., Chromatographia, 8, 55 (1975).

Thomas, H., J. Amer. Chem. Soc., 66, 1664 (1944).

Tiselius, A., Ark. Kem. Mineral. Geol., 14B(22), 1-5 (1940).

Tiselius, A., Ark. Kem. Mineral. Geol., 16A(18), 1-11 (1943).

Tiselius, A., Ark. Kem. Mineral. Geol., 14B(32), 1-8 (1941).

Tuthill, E.J., J. of Chromatogr. Sci., 8, 285 (1970).

Villadsen, J., and Michelsen, M. L., Solution of Differential Equation

Models by Polynomial Approximation, Prentice-Hall, Englewood Cliffs, N.J., 1978.

Wadsworth, M. E., Sep.Sci.and Tech., 22, 711 (1987).

Wankat, P. C., "The Relationship between One Dimensional and Two Dimensional Separation Processes," AIChE J., 23, 859 (1977).

Welstein, H., and Sauer, C., in Ion Exchange Technology, ed. Naden, D., and Streat, M., Ellis Harwood, London, 1984, 463-.471 (1984). 
Witcherle, O., and Coupek, J., Czech. Patent 152, 620 (1974).

Yamamoto, A., "Amino Acids (Survey)," in Kirk Othmer Encyclopedia of Chemical Technology, 3rd ed., V2, Wiley, New York, 1978, 397-410.

Young, J. L., and Yamamoto, M., J. Chromatogr., 78, 221-27 (1973). 

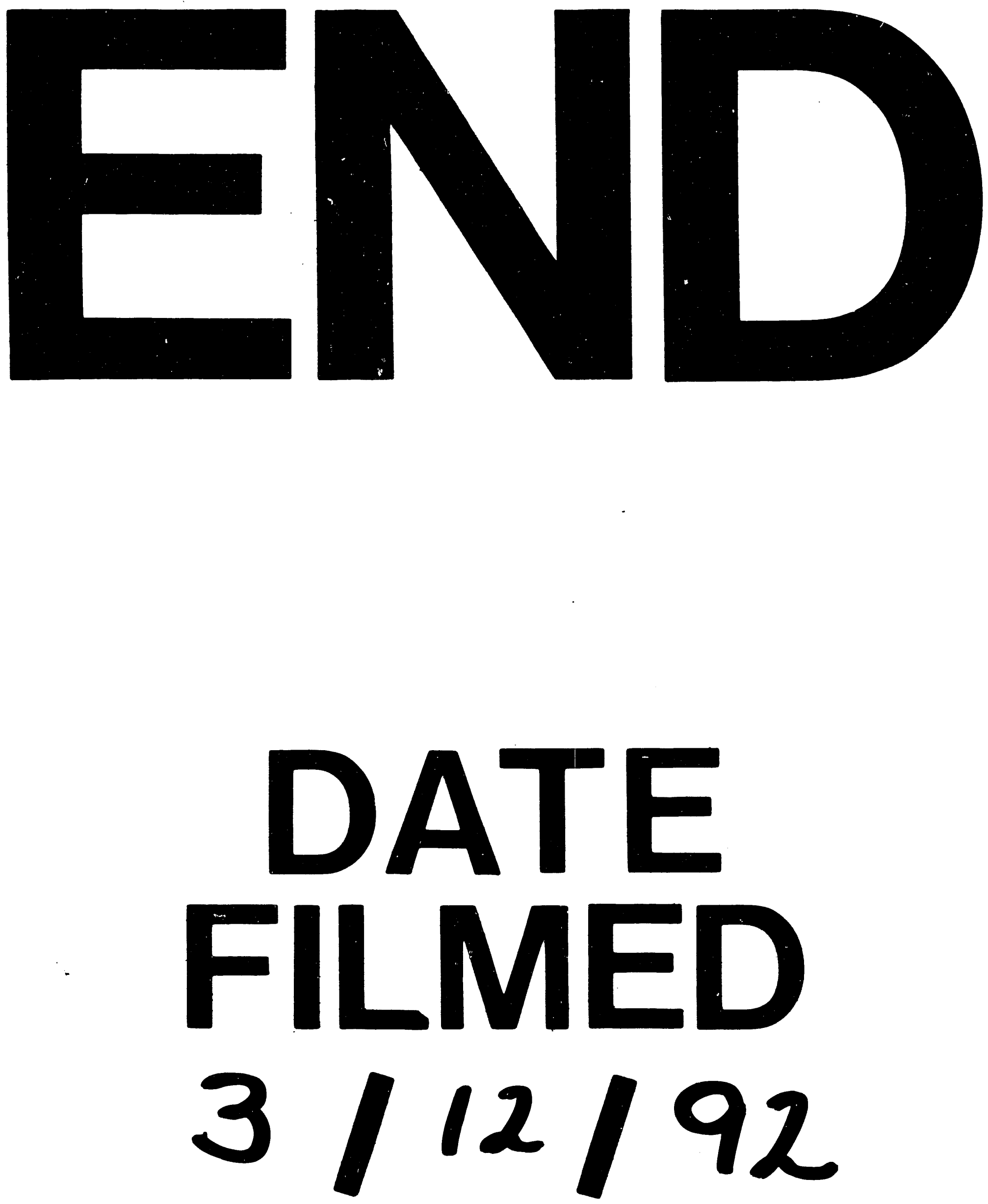
